





\author{
An Index to the \\ Chemical Action of Microorganisms \\ on the Non-Nitrogenous \\ Organic Compounds
}





\section{AN INDEX \\ TO THE CHEMICAL ACTION \\ OF MICROORGANISMS ON THE \\ NON-NITROGENOUS \\ ORGANIC COMPOUNDS}

by

ELLIS I. FULMER, Ph. D.

Professor of Biophysical Chemistry

Iowa State College

and

C. H. WERKMAN, Ph. D.

Associate Professor of Bacteriology, Iowa State College

Assistant Chief in Bacteriology

Iowa Agricultural Experiment Station

Assisted by

Anella Wieben and Calvin R. Breden

Instructors in Chemistry

Iowa State College

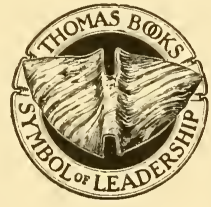

I 930

CHARLES C THOMAS - PUBLISHER

SPRINGFIELD - ILLINOIS BALTIMORE - MARYLAND 
COPYRIGHT, I930, BY CHARLES C THOMAS MANUFACTURED IN THE UNITED STATES 
An Index to the

Chemical Action of Microorganisms on the Non-Nitrogenous

Organic Compounds 



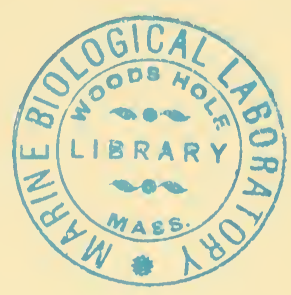

\title{
PREFACE
}

The material presented in this volume is a summary of information in the process of organization for several years, being maintained both in card index and tabular form. These data have been consulted on numerous occasions by various workers in the field of zymology. At the urgent request of these workers, who have found our files and tables useful, we are making this information more generally available in the present form. It is our hope that this index will be of definite value in the rapidly developing field of zymology.

\author{
Ellis I. Fulmer
}

C. H. WERKMAN

Iowa State College

January 24, 1930 



\section{CONTENTS}

Preface......................... vii

INTRODUCTION ........................

I Table One: Index to Microorganisms......... 3

II Table Two: Index to Organic (Non-NitroGenous) Substrates................... 59

III Table Three: Index to Products............. 109

IV References........................ I67

39898 



\section{INTRODUCTION}

$4=18$

In the course of studies on the production of chemicals from agricultural wastes by the action of microorganisms it seemed advisable to obtain a comprehensive view of the chemicals which have been reported as produced by the action of bacteria, yeasts, and molds on various substrates. A survey was made with reference to non-nitrogenous compounds since such compounds furnish the important sources of energy for the organisms and for the zymotechnical production of chemicals on a large scale. This study was further limited to those instances in which a named organism acted on a named substrate to produce a named compound.

To cover the literature completely even with the above limitations would involve so much material as to become unwieldy. The purpose of this survey is to cite references involving a variety of organisms, substrates, products, and authorities; that is, to give a cross section of the field complete enough to introduce the reader to the subject. Hence the authors have purposely omitted much material on the more common types of fermentation with only typical references, while for more uncommon types the treatment has been practically complete. Since the purpose is not primarily historical many of the older references have been omitted and later work cited.

A survey was likewise made of the media and analytical methods for each reference listed. This information will be reserved for a further communication. However, a few general remarks may be pertinent. For example, it is an open question whether in some instances succinic acid is produced from the non-nitrogenous substrate or is a secondary product resulting from the breakdown of the cell or is due to the action upon glutaminic acid. Oxidation and reduction (dehydrogenation and hydrogenation) may in some instances be due to conditions in the medium rather than to direct action of the living organisms. The recent work of Conant and Aston (1928), for example, has shown the production of acetone, carbon dioxide, 
complex crystalline bodies, and gummy substances, by the oxidation of isobutyraldehyde by potassium permanganate or potassium dichromate under the given conditions of temperature and relative concentrations of the reagents. The material at hand likewise shows that one of the immediate needs in the field of zymology is the development and consistent use of adequate analytical methods for the fermentation products.

The names ascribed to the microorganisms by the various investigators have been listed as they are given in the original communication. In case the generic or specific designation has been changed since that time the later name has been crossindexed. The use of an author's name for an organism has been given to identify it. When the statement is made that a certain organism was used to produce definite products, no attempt was made by the authors of this index to judge the purity of the culture. The technique in many instances has not been such as to warrant the conclusions which have been drawn with regard to the ability of the organism to bring about the action reported. It is unfortunate that occasionally investigators will execute a very careful piece of research from a chemical point of view and fail to describe the organism with which the work was done, merely referring to it as a "bacillus." Reference to a number of publications has been omitted for this reason. Frequently a description of an organism has been given which may be adequate for purposes of classification but no name has been attached. The authors wish to emphasize the importance of a proper use of bacterial taxonomy in chemical studies of fermentation.

The results of the literature survey made with the limitations above outlined are presented in three Tables involving the four items: organism, substrate, product and authority. In Table $I$ they are shown in the order named above. This compilation enables the reader to gain an idea of the general chemical actions of a given organism and to compare them with others. In Table 2 the order is substrate, product, organism, authority, enabling the reader at a glance to gain an idea of the various products elaborated from a given substrate by the organism involved. It is of interest to note that the products 
from the pentoses are of the same types as those produced from the hexoses. The order in Table 3 is product, substrate, organism, authority, enabling the reader to find the types of substrate from which a given chemical has been reported as produced by the named organisms. At the end of the paper is given the list of references cited. It is the hope of the authors that this treatment, limited as it is, may be of value ingiving an adequate key to the literature on the chemical changes produced by microorganisms acting on non-nitrogenous organic compounds.

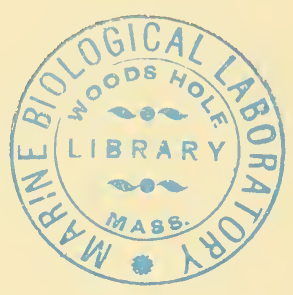





\section{CHAPTER I}

INDEX TO MICROORGANISMS 



\section{CHAPTER I \\ TABLE ONE \\ INDEX TO MICROORGANISMS}

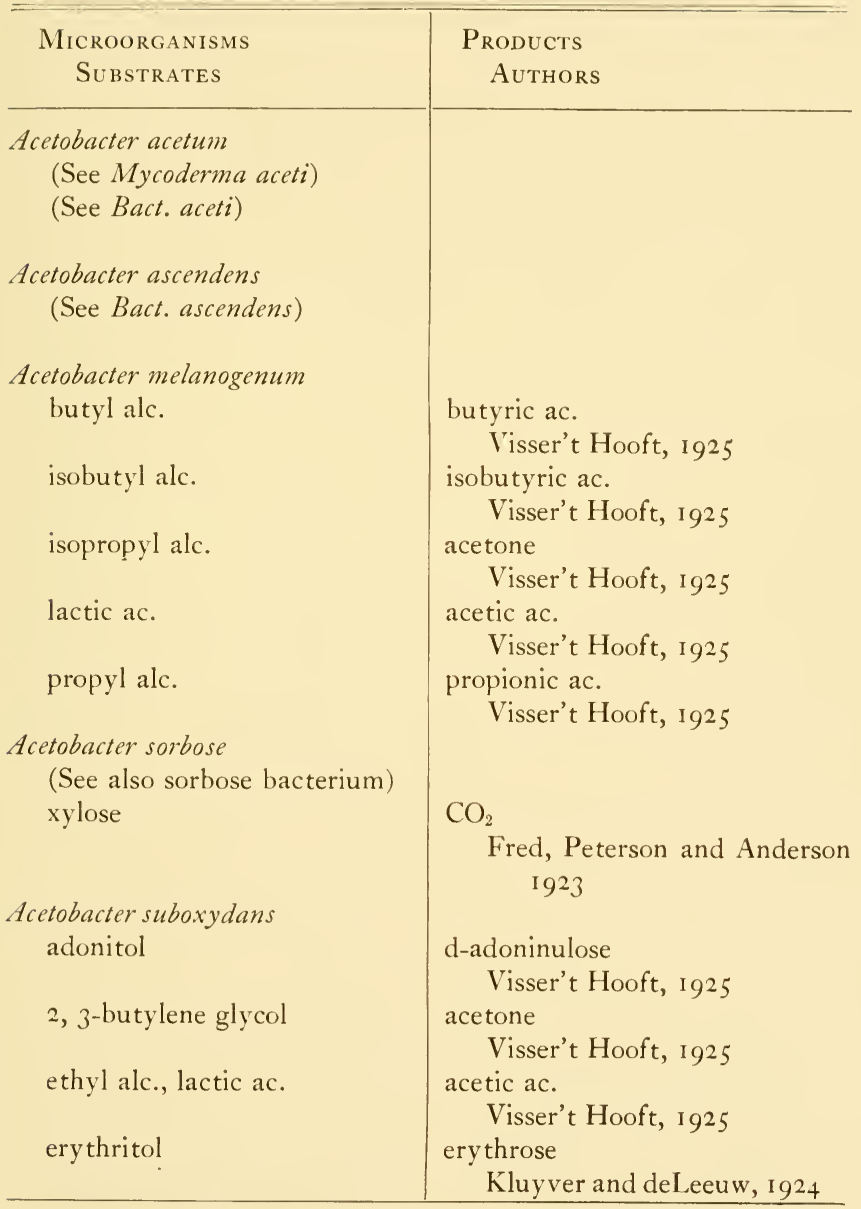




\begin{tabular}{|c|c|}
\hline $\begin{array}{c}\text { Microorganisms } \\
\text { Substrates }\end{array}$ & $\begin{array}{l}\text { Products } \\
\text { Authors }\end{array}$ \\
\hline $\begin{array}{l}\text { Acetobacter suboxydans (cont.) } \\
\text { gluconic ac. } \\
\text { glycerol } \\
\text { glycerol } \\
\text { isopropyl alc. } \\
\text { mannitol } \\
\text { sorbitol }\end{array}$ & $\begin{array}{l}\text { hydroxygluconic ac. } \\
\text { Kluyver and deLeeuw, I } 924 \\
\text { dihydroxy-acetone } \\
\text { Kluyver and deLeeuw, } 1924 \\
\text { dihydroxy-acetone } \\
\text { Brit. patent, } 269,950 \\
\text { acetone } \\
\text { Visser't Hooft, } 1925 \\
\text { levulose } \\
\text { Kluyver and deLeeuw, I924 } \\
\text { sorbose } \\
\text { Kluyver and deLeeuw, I924 }\end{array}$ \\
\hline $\begin{array}{l}\text { Acetobacter sp.? } \\
\text { ethyl alc. } \\
\text { ethyl alc. } \\
\text { ethyl alc. } \\
\text { ethyl alc. } \\
\text { gluconic ac. } \\
\text { glucose }\end{array}$ & 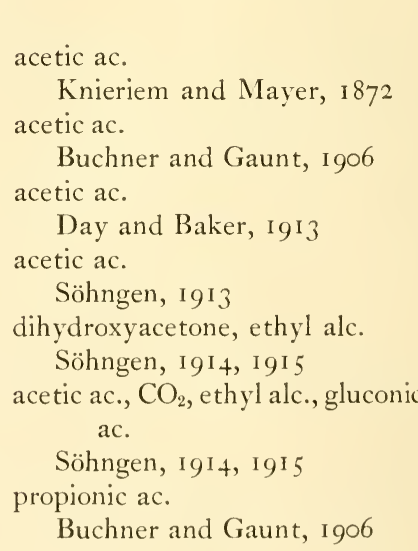 \\
\hline $\begin{array}{l}\text { Acetobacter xylinum } \\
\text { (See also Bact. xylinum) } \\
\text { (See also B. xylinum) } \\
\text { (See also sorbose bacterium) } \\
\text { 2, 3-butylene glycol }\end{array}$ & $\begin{array}{l}\text { acetone } \\
\quad V \text { isser't Hooft, I } 925\end{array}$ \\
\hline
\end{tabular}




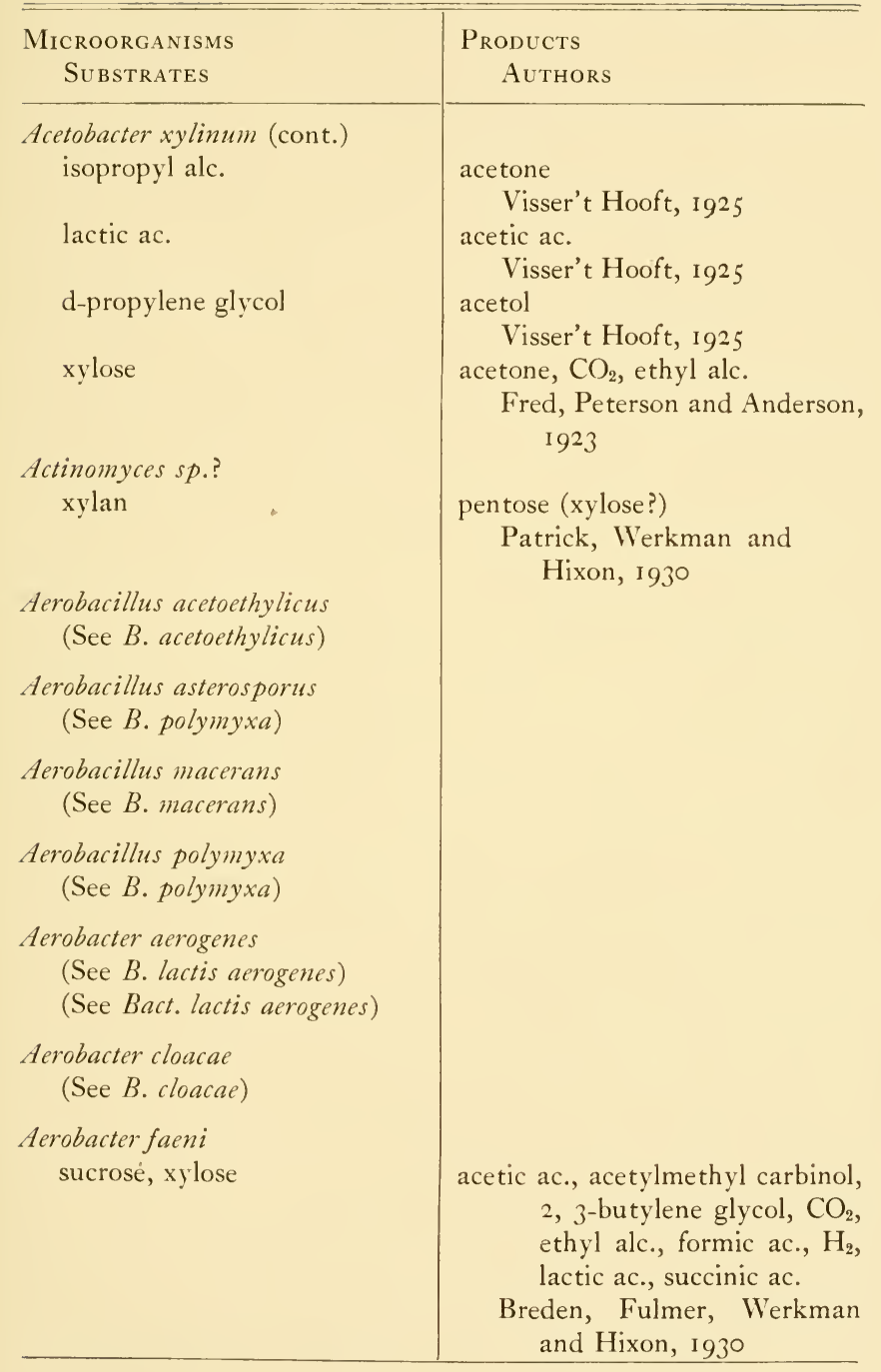




\begin{tabular}{l} 
Microorganisms \\
Substrates \\
\hline Amylobacter butylicus \\
(See also B. amylobacter) \\
glycerol, lactic ac. \\
lactose, maltose, mannitol, \\
starch, sucrose
\end{tabular}

Amylobacter ethylicus sucrose

Amylomyces Rouxii lactic ac.

Aspergillus cellulosae dextrose

Asp. cinnamoneus dextrose

\section{Asp. fumaricus} arabinose

dextrose, gluconic ac., glyceric ac., glycerol, lactic ac., mannitol

galactose

gluconic ac.

glyceric ac.

sucrose

sucrose

\section{Products}

Authors acetic ac., butyl alc., butyric ac. Duclaux, i 895

acetic ac., butyl alc., butyric ac., $\mathrm{CO}_{2}$, ethyl alc., $\mathrm{H}_{2}$, lactic ac. Duclaux, i 895

acetic ac., acetaldehyde, ethyl alc., lactic ac.

Duclaux, 1895

pyruvic ac.

Mazé, I9I6

acetaldehyde

Cohen, 1920

citric ac., gluconic ac.

Falck and Kapur, 1924

fumaric ac., citric ac.

Schreyer, 1928

citric ac.

Schreyer, 1928

fumaric ac., citric ac.

Schreyer, 1928

citric ac.

Schreyer, 1925, 1928

citric ac.

Schreyer, 1928

fumaric ac.

Wehmer, I9I 8

citric ac., fumaric ac., gluconic ac., malic ac.

Wehmer, I 928 


\begin{tabular}{l} 
Microorganisms \\
Substrates \\
\hline $\begin{array}{l}\text { Asp. fuscus } \\
\text { dextrose }\end{array}$ \\
Asp. glaucus \\
glycerol \\
Asp. niger \\
acetic ac. \\
acetic ac., dextrose, starch, \\
tartaric ac. \\
acetonedicarboxylic ac. \\
arabinose, dextrose, galactose, \\
glycerose, levulose, maltose, \\
mannose, sucrose, xylose \\
arabinose, gluconic ac., \\
glycerol, mannitol, quinic \\
ac., saccharic ac. sucrose \\
arabinose, dextrose, glycerol, \\
levulose, mannitol, sucrose \\
butyric ac.
\end{tabular}

Products

Authors

citric ac., gluconic ac.

Falck and Kapur, 1924

hydro-oxymaltol

Traetta-Mosca and Preti, I921

glycollic ac., glyoxylic ac., oxalic ac.

Challenger, Subramaniam and Walker, 1927

oxalic ac.

Wehmer, I $89 \mathrm{I}$

oxalic ac.

Walker, Subramaniam and Challenger, 1927

citric ac.

Amelung, 1927

citric ac., oxalic ac.

Butkewitsch, 1923

gluconic ac.

Bernhauer, I 928

$\beta$-hydroxybutyric ac., acetoacetic ac., acetone

Koppock, Subramaniam and

cinnamic ac. Walker, I 928

styrol

Herzog and Ripke, I908

citric ac.

citric ac. acetone, glyoxylic ac., malonic ac., oxalic ac.

Challenger, Subramaniam and Walker, 1927 acetonedicarboxylic ac.

Walker, Subramaniam and Challenger, 1927 


\begin{tabular}{|c|c|}
\hline $\begin{array}{l}\text { Microorganisms } \\
\text { SubStrates }\end{array}$ & $\begin{array}{l}\text { Products } \\
\text { Authors }\end{array}$ \\
\hline \multicolumn{2}{|l|}{ Asp. niger (cont.) } \\
\hline citric ac. & $\begin{array}{l}\text { glycollic ac. } \\
\text { Challenger, Subramaniam and } \\
\text { Walker, } 1927\end{array}$ \\
\hline citric ac. & $\begin{array}{l}\text { oxalic ac. } \\
\text { Challenger, Subramaniam and } \\
\text { Walker, } 1927\end{array}$ \\
\hline $\begin{array}{l}\text { dextrin, dextrose, glycerol, } \\
\text { inulin, lactose, mannitol, } \\
\text { sucrose }\end{array}$ & $\begin{array}{l}\text { citric ac., oxalic ac. } \\
\text { Elfving, Ig I9 }\end{array}$ \\
\hline dextrose & $\begin{array}{l}\text { acetic ac., oxalic ac. } \\
\text { Heinze, I903 }\end{array}$ \\
\hline dextrose, sucrose & $\begin{array}{l}\text { citric ac. } \\
\quad \text { Bernhauer, } 1928\end{array}$ \\
\hline dextrose & $\begin{array}{l}\text { citric ac., gluconic ac. } \\
\text { Falck and Kapur, } 1924\end{array}$ \\
\hline dextrose & $\begin{array}{c}\text { citric ac., oxalic ac. } \\
\text { Wehmer, } 1924\end{array}$ \\
\hline dextrose & $\begin{array}{l}\text { ethyl alc., } \mathrm{CO}_{2} \\
\text { Kostytschew, I } 907\end{array}$ \\
\hline dextrose & $\begin{array}{l}\text { gluconic ac. } \\
\text { Müller, I } 925\end{array}$ \\
\hline dextrose & $\begin{array}{l}\text { oxalic ac. } \\
\text { Wehmer, I } 897\end{array}$ \\
\hline fumaric ac. & $\begin{array}{l}\text { 1-malic ac. } \\
\text { Challenger and Klein, I929 }\end{array}$ \\
\hline gluconic ac. & $\begin{array}{l}\text { citric ac., saccharic ac. } \\
\text { Walker, Subramaniam and } \\
\text { Challenger, I927 }\end{array}$ \\
\hline gluconic ac. & $\begin{array}{l}\text { citric ac., oxalic ac. } \\
\text { Wehmer, I925 }\end{array}$ \\
\hline oxalic ac. & $\begin{array}{l}\text { glycollic ac. } \\
\text { Challenger, Subramaniam and } \\
\text { Walker, } 1927\end{array}$ \\
\hline phloridzin & $\begin{array}{l}\text { phloroglucinol, dextrose } \\
\text { Boas, I9 } 6\end{array}$ \\
\hline
\end{tabular}




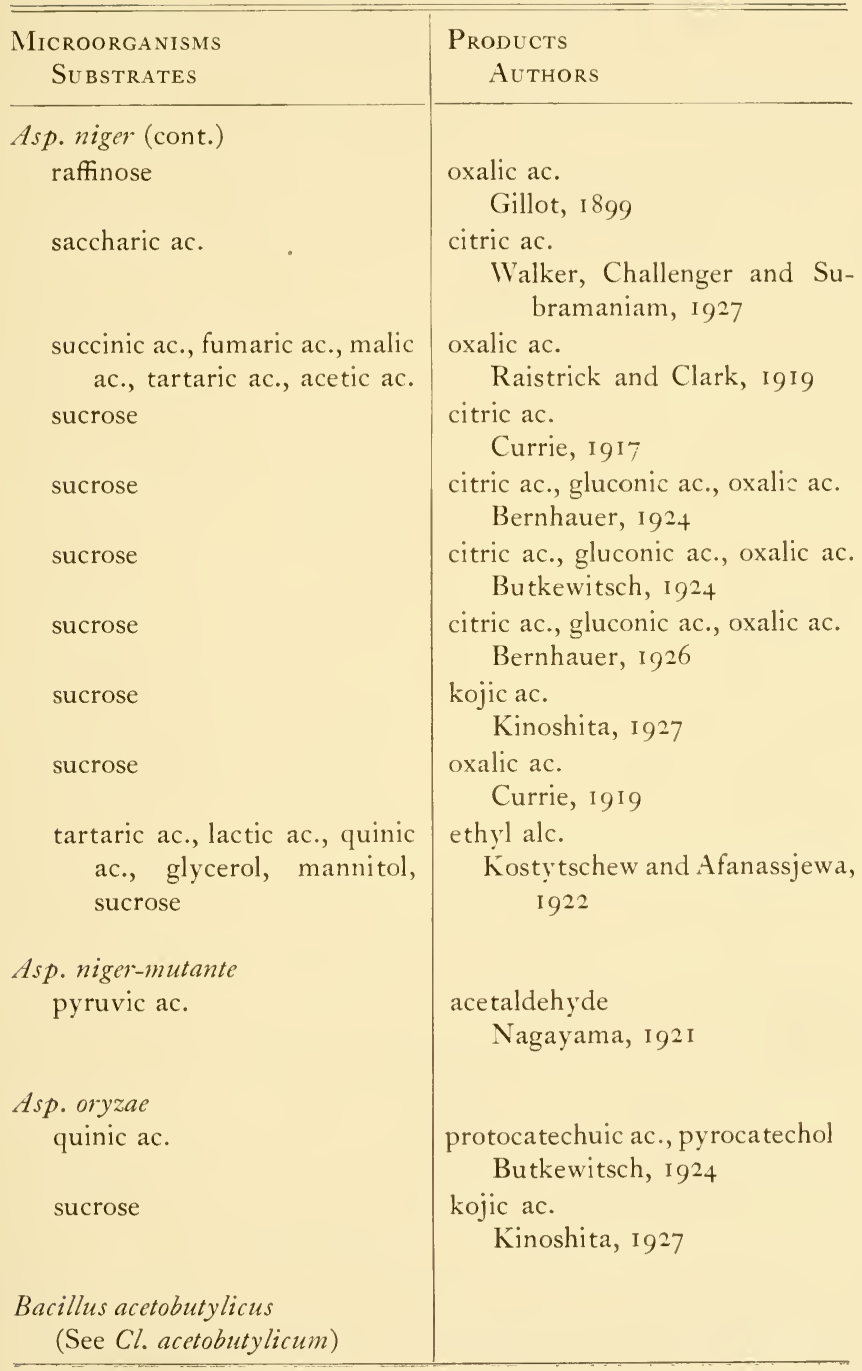




\begin{tabular}{|c|c|}
\hline $\begin{array}{c}\text { Microorganisms } \\
\text { Substrates }\end{array}$ & $\begin{array}{l}\text { Products } \\
\text { Authors }\end{array}$ \\
\hline $\begin{array}{l}\text { B. aceti } \\
\text { arabinose, dextrose, erythritol } \\
\text { ethyleneglycol, glycerol, } \\
\text { glycollic ac., isobutyric } \\
\text { ac., isolichenin, levulose, } \\
\text { malonic ac., rhamnose, } \\
\text { sucrose }\end{array}$ & $\begin{array}{l}\text { oxalic ac. } \\
\text { Banning, } 1902 \\
.\end{array}$ \\
\hline $\begin{array}{l}\text { B. aceti (Hansen) } \\
\text { ethyl alc. } \\
\text { propyl alc. }\end{array}$ & $\begin{array}{l}\text { acetic ac. } \\
\text { Buchner and Gaunt, I906 } \\
\text { propionic ac. } \\
\text { Buchner and Gaunt, } 1906\end{array}$ \\
\hline $\begin{array}{l}\text { B. aceti viscosum } \\
\text { dextrose } \\
\text { ethyl alc. }\end{array}$ & $\begin{array}{l}\text { gluconic ac. } \\
\text { Day and Baker, 19I3 } \\
\text { acetic ac. } \\
\text { Day and Baker, } 19 \text { I } 3\end{array}$ \\
\hline $\begin{array}{l}\text { B. acetigenum } \\
\text { arabinose, dextrose, } \\
\text { glycollic ac. } \\
\text { dextrose }\end{array}$ & $\begin{array}{l}\text { oxalic as. } \\
\text { Banning, } 1902 \\
\text { gluconic ac. } \\
\text { Henneberg, } 1898\end{array}$ \\
\hline $\begin{array}{l}\text { B. acetoethylicum } \\
\text { arabinose, dextrose, galactose, } \\
\text { glycerol, lactic ac., levu- } \\
\text { lose, maltose, mannose, } \\
\text { raffinose, starch, sucrose, } \\
\text { xylose } \\
\text { dextrose, maltose }\end{array}$ & $\begin{array}{l}\text { acetone, ethyl alc., formic ac. } \\
\text { Northrop, Ashe and Morgan, } \\
\text { I9I9 }\end{array}$ \\
\hline
\end{tabular}

* While $B$. usually designates Bacillus, this symbol is used occasionally for Bacterium, and leads to confusion. The authors have used the designation of the authorities cited. 
Microorganisms

Substrates

B. acetoethylicum (cont.) dextrose, starch, sucrose, xylose

starch

B. acetoethylicus dextrose

starch, sucrose

B. acetosum arabinose, dextrin, dextrose, erythritol, ethyleneglycol, glycollic ac., malonic ac. dextrose

B. acidi-laeurolactici sucrose

B. acidi propionici

(See Propionibacterium pentosaceum)

B. acidi propionici lactic ac.

\section{Bacillus aerogenes}

(See B. lactis aerogenes)
Products

Authors

acetic ac., acetone, ethyl alc., formic ac., lactic ac.

Arzberger, Peterson and Fred, I 920

acetaldehyde

Peterson and Fred, 1920

acetic ac., acetylmethyl carbinol, acetone, 2, 3-butylene glycol, $\mathrm{CO}_{2}$, ethyl alc., formic ac., $\mathrm{H}_{2}$, lactic ac.

Donker, 1926 acetone, ethyl alc.

Bakonyi, 1926

oxalic ac.

Banning, 1902

gluconic ac.

Henneberg, I 898

1-lactic ac.

Schardinger, I 890

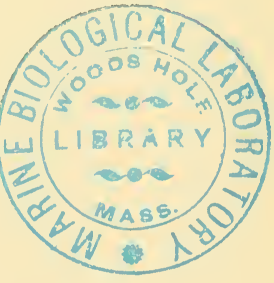

acetic ac., $\mathrm{CO}_{2}$, propionic ac.

von Fruedenreich and Jensen, I 906 


Microorganisms
Substrates

$B$. aerogenes dextrose

B. amaracrylus glycerol

$B$. amarificans

(See B. lactis)

B. amylobacter

(See Clostridium butyricum) (See Granulobacter butylicum)

B. anthracis

maltose, sucrose

starch

B. ascendens

(See also Acetobacter ascendens) (See also Bart. ascendens)

B. ascendens acetaldol

arabinose, dextrose, ethylene glycol, glycerol, glycollic ac., malonic ac., rhamnose methylethyl-acetaldehyde
Products

Authors

acetic ac., acetylmethyl carbinol, 2, 3-butyleneglycol, $\mathrm{CO}_{2}$, ethyl alc., formic ac., $\mathrm{H}_{2}$, lactic ac., succinic ac.

Scheffer, 1928

acrylaldehyde

Voisenet, I9I 8

acetylmethyl carbinol

Lemoigne, I9I9

dextrose

Maumus, I 893

$\beta$-butylene glycol, $\beta$-hydroxybutyric ac.

Binder-Kotrba, I 926

oxalic ac.

Banning, 1902

l-amyl alc., valeric ac.

Neuberg and Simon, 1926 
Microorganisms

Substrates

B. butylicus

(See also B. amylobacter)

dextrose, glycerol

dextrose, glycerol, lactic ac., propionic ac.

glycerol

glycerol, mannitol

glycerol, mannitol, sucrose

B. butyricus

(See B. amylobacter)

B. casei

dextrose

B. casei

lactic ac., succinic ac.

B. casei E. sucrose

B. caucasicus

(See Bact. caucasicum)

$B$. cellulosae dissolvens cellulose

\section{Products}

Authors

acetic ac., n-butyl alc., n-butyric ac., formic ac., lactic ac.

Buchner and Meisenheimer, I 908

butyric ac., butyl alc., caproic ac., caprylic ac.

Neuberg and Arinstein, I92 I n-amyl alc., n-butyl alc., n-propyl alc.

Morin, 1887

butyl alc.

Emmerling, I 897

butyl alc., butyric ac., lactic ac.

Fitz, I 882

lactic ac.

Virtanen and Karström, 1928

acetic ac., propionic ac.

Orla-Jensen, 1904

i-lactic ac.

Virtanen, Wichmann and Lindström, 1927

acetic ac., butyric ac., $\mathrm{CO}_{2}$, ethyl alc., $\mathrm{H}_{2}$

Khouvine, 1923 
Microorganisms

Substrates

B. cloacae

arabinose, galactose, isodulcite, levulose, mannose dextrose, mannitol

dextrose

dextrose, mannitol

B. coli

(See also Bact. coli)

(See also Bact. coli communis) dextrose

dextrose

dextrose

dextrose, galactose, lactose, mannitol, mannose, sucrose dextrose, mannitol

dextrose

dextrose
Products

Authors

acetylmethyl carbinol

Harden and Norris, I9I 2 acetylmethyl carbinol, 2, 3-butylene glycol

Thompson, 191 2 acetylmethyl carbinol, 2, 3-butylene glycol, $\mathrm{CO}_{2}$, ethyl alc., formic ac., $\mathrm{H}_{2}$, lactic ac.

Scheffer, 1928

acetic ac., ethyl alc., $\mathrm{CO}_{2}$, formic ac., lactic ac., succinic ac.

Thompson, I91 2

pyruvic ac.

Aubel, I926

acetic ac., ethyl alc., l-lactic ac., succinic ac.

Grimbert, I 896

acetic ac., $\mathrm{CO}_{2}$, ethyl alc., formic ac., $\mathrm{H}_{2}$, lactic ac., succinic ac.

Scheffer, I928

lactic ac.

Péré, 1898

acetic ac., ethyl alc., formic ac., lactic ac., succinic ac.

Thompson, I9I 2

acetic ac., $\mathrm{CO}_{2}$, ethyl alc., formic ac., $\mathrm{H}_{2}$, lactic ac., succinic ac.

Virtanen and Simola, 1927 lactic ac.

Neuberg and Gorr, 1926 


Microorganisms
Substrates

B. coli (cont.) lactose

malic ac.

methylglyoxal

pyruvic ac.

succinic ac.

sucrose

B. coli communis

(See also B. coli)

(See also Bact. coli)

(See also Bact. coli communis) arabinose, dextrose, d-galactose, levulose, mannitol

citric ac.

dextrose

dextrose

dextrose

dextrose

\section{Products}

Authors

acetic ac., ethyl alc., l-lactic ac., succinic ac.

Grimbert, 1896

fumaric ac.

Quastel and Whetham, I924 lactic ac.

Neuberg and Gorr, 1926

acetic ac., formic ac., glycollic ac., lactic ac.

Cambier and Aubel, 1922 fumaric ac.

Quastel and Whetham, 1924 lactic ac., methylglyoxal

Virtanen and Simola, 1927

acetic ac., $\mathrm{CO}_{2}$, ethyl alc., $\mathrm{H}_{2}$, lactic ac., succinic ac.

Harden, I90 I

acetic ac., $\mathrm{CO}_{2}$, ethyl alc., succinic ac.

Grey, 1923

acetic ac., $\mathrm{CO}_{2}$, ethyl alc., formic ac., $\mathrm{H}_{2}$, lactic ac., succinic ac.

Grey, I9I 8

acetaldehyde

Grey, I9I 3

acetic ac., $\mathrm{CO}_{2}, \mathrm{H}_{2}$, ethyl alc., lactic ac.

Harden, 1899

acetic ac., $\mathrm{CO}_{2}$, ethyl alc., formic ac., $\mathrm{H}_{2}$, lactic ac., succinic ac.

Grey, I920 
dextrose

dextrose, mannitol

dextrose

dextrose

dextrose

dextrose, gluconic ac., mannitol, saccharic ac.

formic ac.

fumaric ac.

glucosamine

glycerol

lactic ac.

malic ac.

malic ac.

malonic ac.

\section{Products}

Authors

acetic ac., $\mathrm{CO}_{2}$, ethyl alc., formic ac., $\mathrm{H}_{2}$, lactic ac., succinic ac.

Grey and Young, I92 I acetic ac., lactic ac., succinic ac. Young, 1924 acetic ac., $\mathrm{CO}_{2}$, ethyl alc., $\mathrm{H}_{2}$, lactic ac., succinic ac.

Grey, I9I 8

formic ac.

Franzen and Kahlenberg, 1916 1-lactic ac.

Kimura, 1928

$\mathrm{CO}_{2}$, lactic ac.

Goto, 1925

acetic ac., formic ac., succinic ac.

Kay, 1926

$\mathrm{CO}_{2}, \mathrm{H}_{2}$

Franzen and Kahlenberg, 1916 succinic ac.

Quastel, Stephenson and Whetham, 1925

d-lactic ac.

Kimura, 1928

acetic ac., $\mathrm{CO}_{2}$, ethyl alc., formic ac., lactic ac., succinic ac.

Grey, I923

pyruvic ac.

Quastel, Stephenson and Whetham, I 925 succinic ac., fumaric ac.

Quastel and Whetham, 1924 acetic ac., ethyl alc., succinic ac.

Grey, 1923 acetic ac.

Grey, I923 


Microorganisms
Substrates

B. coli communis (cont.) succinic ac.

tartaric ac.

B. cylindrosporus

(See B. lactis)

B. Delbriicki dextrin, dextrose, maltose, sucrose methylglyoxal

phenylglyoxal

B. dioxyacetonicum glycerol

B. dysenteriae, Shiga-Kruse dextrose

dextrose

B. ethaceticus arabinose

dextrose, mannitol glycerol, mannitol

mannitol

\section{Products}

Authors

acetic ac., formic ac., $\mathrm{H}_{2}$

Grey, 1923

acetic ac., ethyl alc., succinic ac.

Grey, I 923

1-lactic ac.

Henneberg, I 903

d, 1-lactic ac.

Henneberg, 1903

d-mandelic ac.

Henneberg, 1903

dihydroxyacetone

Virtanen and Barlund, I926

acetic ac., ethyl alc., formic ac., lactic ac., succinic ac.

Scheffer, I 928

acetic ac., acetaldehyde, ethyl alc., formic ac., succinic ac.

Bergh, I928

acetic ac., ethyl alc., formic ac., succinic ac.

Frankland and MacGregor, I 892

acetic ac., $\mathrm{CO}_{2}$, ethyl alc., $\mathrm{H}_{2}$

Frankland and Lumsden, I 892 acetic ac., ethyl alc., formic ac., succinic ac.

Frankland and Fox, I889 acetic ac., ethyl alc.

Frankland, Stanley and Frew, I 89 I 
Microorganisms

Substrates

B. ethacetosuccinicus

lactic ac.

dulcitol, mannitol

B. fuorescens

malic ac.

B. Freundii

(See also Bact. Freundii)

dextrose

B. globigii

(See B. mesentericus ruber as a possible synonym)

B. granulobacter pectinovorum

(See also B. amylobacter)

(See also B. butylicus)

(See also B. butyricum)

(See also $\mathrm{Cl}$. pastorianum)

B. granulobacter pectinovorum

arabinose, dextrose galactose, mannitol, starch, xylose

arabinose, xylose

B. granulobacter pectinovorum

Weizmann

dextrose
Products

Authors

acetic ac., ethyl alc., formic ac.

Mazé, I9I3

acetic ac., $\mathrm{CO}_{2}$, ethyl alc., $\mathrm{H}_{2}$, succinic ac.

Frankland and Frew, I 892

pyruvic ac.

Beijerinck and Folpmers, 1916

acetic ac., $\mathrm{CO}_{2}$, ethyl alc., formic ac., $\mathrm{H}_{2}$, lactic ac., succinic ac.

Scheffer, I 929

acetic ac., acetone, butyl alc., butyric ac., lactic ac.

Speakman, 1923 acetone, butyl alc., $\mathrm{CO}_{2}$

Peterson, Fred and Schmidt, I $9^{2} 4$

acetic ac., acetylmethyl carbinol, acetone, butyl alc., butyric ac., $\mathrm{CO}_{2}$, ethyl alc., formic ac., $\mathrm{H}_{2}$, lactic ac.

Donker, 1926 


\begin{tabular}{|c|c|}
\hline $\begin{array}{c}\text { Microorganisms } \\
\text { Substrates }\end{array}$ & $\begin{array}{l}\text { Products } \\
\text { Authors }\end{array}$ \\
\hline $\begin{array}{l}\text { B. graveolens } \\
\text { (See } B \text {. mesentericus-vulgatus } \\
\text { as a possible synonym) }\end{array}$ & \\
\hline $\begin{array}{l}\text { B. giintheri } \\
\text { dextrose }\end{array}$ & $\begin{array}{c}\text { acetic ac., lactic ac. } \\
\text { Hucker, I } 928\end{array}$ \\
\hline $\begin{array}{l}\text { B. herbicola aurem } \\
\text { arabinose, xylose }\end{array}$ & $\begin{array}{l}\mathrm{CO}_{2} \\
\text { Fred, Peterson and Anderson, } \\
\quad \text { I } 923\end{array}$ \\
\hline $\begin{array}{l}\text { B. industrium } \\
\text { acetic ac., dextrose, ery- } \\
\text { thritol, ethylene glycol, } \\
\text { isolichenin, mannitol }\end{array}$ & $\begin{array}{l}\text { oxalic ac. } \\
\text { Banning, I902 }\end{array}$ \\
\hline $\begin{array}{l}\text { B. invertenti-acetici } \\
\text { sucrose }\end{array}$ & $\begin{array}{l}\text { acetic ac., acetone, ethyl alc., } \\
\text { lactic ac. } \\
\text { Mezzadroli, } 9^{17}\end{array}$ \\
\hline $\begin{array}{l}\text { B. invertenti-lattici } \\
\text { sucrose }\end{array}$ & $\begin{array}{c}\text { acetic ac., lactic ac. } \\
\text { Mezzadroli, I917 }\end{array}$ \\
\hline $\begin{array}{l}\text { B. Kiitzingianum } \\
\text { arabinose, dextrin, dextrose, } \\
\text { ethylene glycol, galactose, } \\
\text { glycollic ac., isobutyric ac., } \\
\text { isolichenin, lactic ac., } \\
\text { malonic ac. } \\
\text { acetic ac. } \\
\text { acetic ac. } \\
\text { butyl alc. }\end{array}$ & $\begin{array}{l}\mathrm{CO}_{2}, \\
\text { Seifert, I } 897 \\
\mathrm{CO}_{2} \text {, } \\
\text { Mayer, I } 898 \\
\text { butyric ac. } \\
\text { Seifert, I } 897\end{array}$ \\
\hline
\end{tabular}




\begin{tabular}{|c|c|}
\hline $\begin{array}{c}\text { Microorganisms } \\
\text { Substrates }\end{array}$ & $\begin{array}{l}\text { Products } \\
\text { Authors }\end{array}$ \\
\hline $\begin{array}{l}\text { B. Kïtzingianum (cont.) } \\
\text { butyl alc. } \\
\text { dextrose } \\
\text { dextrose } \\
\text { ethyl alc. } \\
\text { ethylene glycol } \\
\text { ethylene glycol } \\
\text { propyl alc. }\end{array}$ & $\begin{array}{l}\text { butyric ac. } \\
\text { Mayer, I } 898 \\
\text { gluconic ac. } \\
\text { Seifert, I } 897 \\
\text { gluconic ac. } \\
\text { Mayer, I } 898 \\
\text { acetic ac. } \\
\text { Mayer, I } 898 \\
\text { glycollic ac. } \\
\text { Seifert, I } 897 \\
\text { glycollic ac. } \\
\text { Mayer, I } 898 \\
\text { propionic ac. } \\
\text { Mayer, I } 898\end{array}$ \\
\hline $\begin{array}{l}\text { B. lactis } \\
\text { i-inositol }\end{array}$ & $\begin{array}{l}\text { acetic ac., ethyl alc., } \mathrm{CO}_{2} \text {, suc- } \\
\text { cinic ac. } \\
\text { Hewitt and Steabben, I92 I }\end{array}$ \\
\hline $\begin{array}{l}\text { B. lactis acidi } \\
\text { dextrin, dextrose, maltose, } \\
\text { sucrose } \\
\text { lactose }\end{array}$ & $\begin{array}{l}\text { 1-lactic ac. } \\
\text { Henneberg, } 1903 \\
\text { d-lactic ac. } \\
\text { Henneberg, I903 }\end{array}$ \\
\hline $\begin{array}{l}\text { arabinose, dextrose, galactose, } \\
\text { isodulcite, mannose } \\
\text { dextrose }\end{array}$ & $\begin{array}{l}\text { acetic ac., 2, 3-butylene glycol, } \\
\text { succinic ac. } \\
\text { Harden and Norris, I9 } 2 \\
\text { acetylmethyl carbinol, 2, 3-buty- } \\
\text { lene glycol } \\
\text { Harden and Norris, I9 I } 2 \\
\text { acetylmethyl carbinol, 2, 3-buty- } \\
\text { lene glycol } \\
\text { Harden and Norris, I9 } 2 \\
\text { acetaldehyde } \\
\text { Kumagawa, I922 }\end{array}$ \\
\hline
\end{tabular}




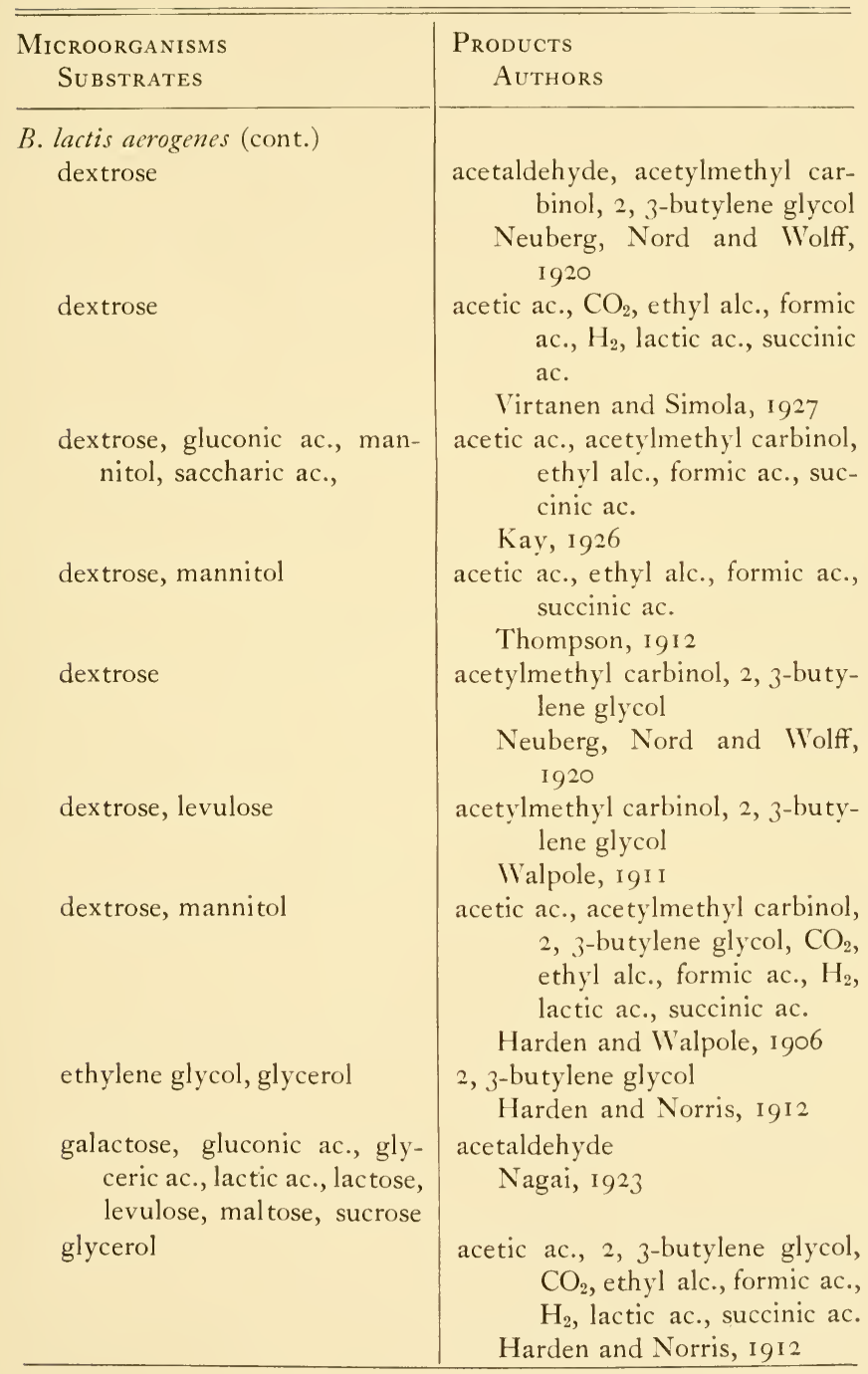




Microorganisms
Substrates

Products

Authors

B. lactic aerogens (cont.) glycerol

inositol

lactose

malic ac.

xylose

B. macerans

starch, sucrose

B. of malignant oedema dextrose

B. mesentericus-ruber

lactic ac.

B. mesentericus vulgatus dextrose

mannitol

B. mobilis

lactic ac.

B. orthobutylicus (Grimbert)

(See Cl. butyricum)

(See B. Granulobacter butyricum) acetaldehyde

Kumagawa, 1922

acetaldehyde, lactic ac., $\mathrm{CO}_{2}$, succinic ac.

Kumagawa, 1922

acetic ac., succinic ac.

Emmerling, 1900

acetic ac., succinic ac.

Emmerling, I 899

acetic ac., $\mathrm{CO}_{2}$, ethyl alc., $\mathrm{H}_{2}$, lactic ac.

Fred and Peterson, I920

acetone, ethyl alc.

Bakonyi, 1926

i-lactic ac., ethyl alc.

Kerry and Frankel, I 890

2, 3-butylene glycol

Lemoigne, I9I3

acetylmethyl carbinol

Harden and Norris, I91 2

acetylmethyl carbinol

Harden and Norris, 1912

acetic ac., propionic ac.

Jensen, 1904 


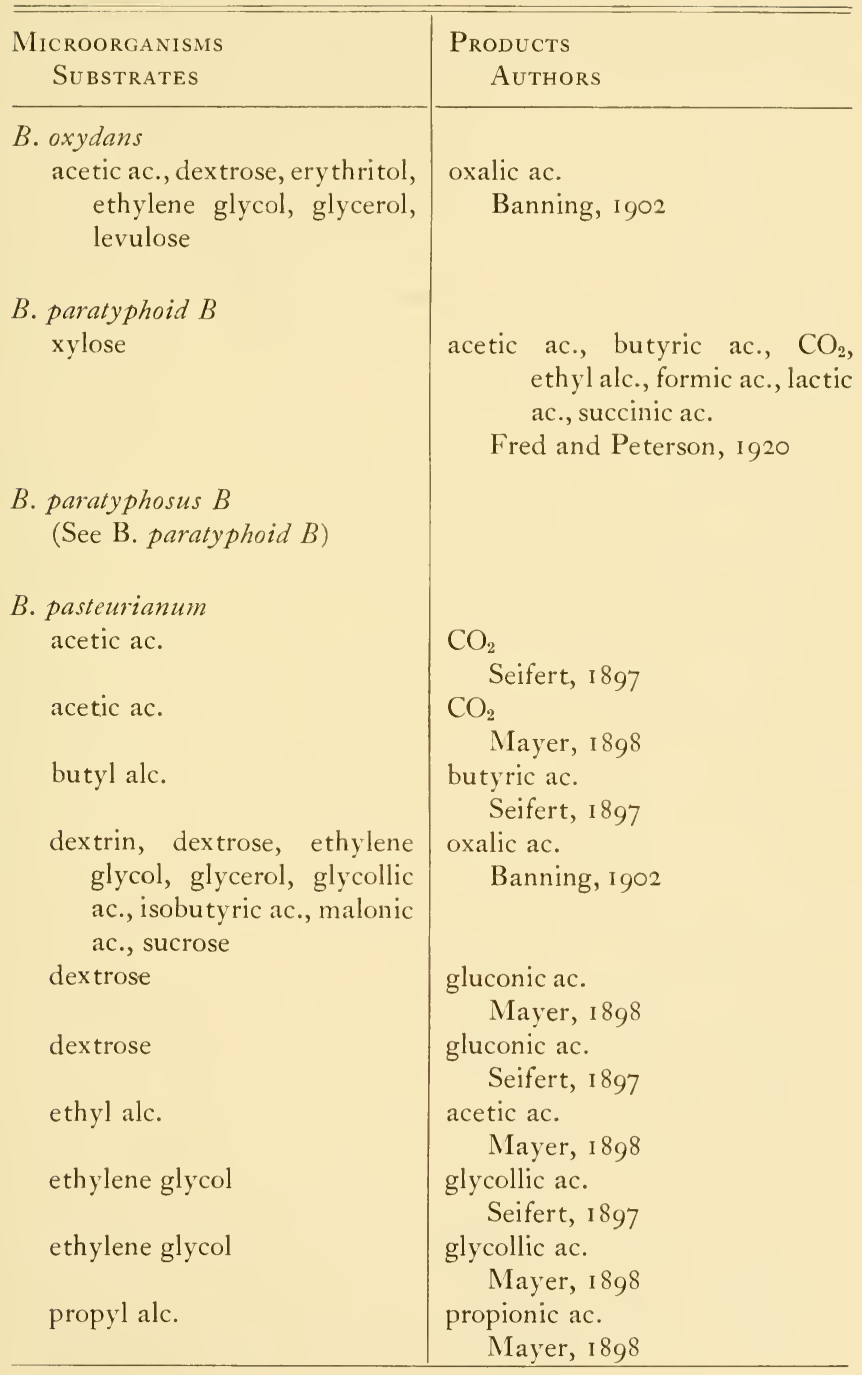


Microorganisms

Substrates

B. polymyxa

dextrose

B. prodigiosus

dextrose

dextrose

glucosamine

B. propionicus

dextrose

methylglyoxal

B. proteus

dextrose

B. proteus vulgatus

pyruvic ac.

B. pyocyaneus

(See also Bact.pyocyaneus) acetaldehyde

acetic ac.

acetone

ethyl alc.

\section{Products}

Authors

acetic ac., acetylmethyl carbinol, acetone, butyl alc., 2, 3butylene glycol, $\mathrm{CO}_{2}$, ethyl alc., formic ac., $\mathrm{H}_{2}$, lactic ac.

Donker, 1926

l-lactic ac.

Kimura, I 928

formic ac.

Franzen and Egger, I9I 2

1-lactic ac.

Kimura, I928

lactic ac.

Neuberg and Gorr, I926 lactic ac.

Neuberg and Gorr, 1926

acetylmethyl carbinol, 2, 3-butylene glycol

Lemoigne, 1923

acetic ac., lactic ac., glycollic ac.

Cambier and Aubel, I922

acetic ac.

Supniewski, 1923

formaldehyde, formic ac.

Supniewski, 1923

acetic ac., formic ac.

Supniewski, 1923

acetaldehyde, acetic ac.

Supniewski, I923 


\begin{tabular}{c}
\hline $\begin{array}{c}\text { Microorganisms } \\
\text { Substrates }\end{array}$ \\
\hline
\end{tabular}

Products

B. pyocyaneus (cont.)

fumaric ac.

Authors

fumaric ac.

glycerol

lactic ac.

lactic ac.

methyl alc.

pyruvic ac.

pyruvic ac.

succinic ac.

acetic ac., pyruvic ac.

Quastel, I924

pyruvic ac.

Quastel, Stephenson and

Whetham, I925

$\mathrm{CO}_{2}$, glyceric ac., lactic ac.

Supniewski, I923

acetaldehyde, acetic ac., pyruvic ac.

Supniewski, I923

pyruvic ac.

Quastel, Stephenson and Whetham, I925

formic ac.

Supniewski, I923 acetic ac., formic ac.

Cambier and Aubel, I922 lactic ac.

Aubel, I924

propionic ac.

Quastel, I924

B. spongiosus

raffinose, sucrose

B. suaveolens

starch

succinic ac.

B. subtilis

dextrose

dextrose

arabin

Ruhland, I906

acetaldehyde, acetic ac., butyric ac., ethyl alc., formic ac.

Sclavo and Gosio, I89 I

fumaric ac.

Quastel and Whetham, I924

acetylmethyl carbinol

Harden and Norris, I 9 I 2

d- or dl-lactic ac.

Kimura, I928 


Microorganisms
Substrates

B. subtilis (cont.)

dextrose

glucosamine

glycerol

glycerol

lactic ac.

mannitol

starch

sucrose

B. tarbellicus

(See Thermobacillus tarbellicus)

B. tartricus

dextrose, lactose, maltose, mannitol, sucrose

tartaric ac.

B. thermoamylolyticus

starch

B. thermobutyricus

dextrose

\section{Products}

Authors

butyric ac., $\mathrm{CO}_{2}$, lactic ac., $\mathrm{H}$, mannitol

Vandevelde, $188_{4}$

H-lactic ac.

Kimura, 1928

butyric ac., lactic ac., succinic ac.

Vandevelde, I $88_{4}$

pyruvic ac.

Aubel, I92 I

acetylmethyl carbinol, 2, 3-butylene glycol

Lemoigne, I923

acetylmethyl carbinol

Harden and Norris, I9I 2

acetic ac., butyric ac., ethyl alc., succinic ac.

Fitz, 1878

acetylmethyl carbinol, 2, 3-butylene glycol

Lemoigne, I91 2

acetic ac., acetylmethyl carbinol, $\mathrm{CO}_{2}$, ethyl alc., $\mathrm{H}_{2}$, lactic ac., succinic ac.

Grimbert, I90 I

acetic ac., $\mathrm{CO}_{2}, \mathrm{H}_{2}$, succinic ac.

Grimbert, 1898

maltose

Coolhaas, 1928

butyric ac.

Coolhaas, 1928 


\begin{tabular}{|c|c|}
\hline $\begin{array}{c}\text { Microorganisms } \\
\text { Substrates }\end{array}$ & $\begin{array}{l}\text { Products } \\
\text { Authors }\end{array}$ \\
\hline $\begin{array}{l}\text { B. typhosum } \\
\text { dextrose }\end{array}$ & $\begin{array}{l}\text { acetic ac., ethyl alc., formic ac. } \\
\text { lactic ac., succinic ac. } \\
\text { Scheffer, } 1928\end{array}$ \\
\hline $\begin{array}{l}\text { B. typhosus } \\
\text { dextrose }\end{array}$ & $\begin{array}{l}\text { acetic ac., formic ac., ethyl alc. } \\
\text { lactic ac., succinic ac. } \\
\text { Harden, Igor } \\
\text { acetic ac., butyric ac., ethyl alc. } \\
\text { formic ac., lactic ac. } \\
\text { succinic ac. } \\
\text { Fred and Peterson, 1920 }\end{array}$ \\
\hline $\begin{array}{l}\text { B. vulgatus } \\
\text { sucrose } \\
\text { xylose }\end{array}$ & $\begin{array}{l}\text { gum levan } \\
\text { Owen, } \mathrm{I}^{2} 3 \\
\text { acetone, } \mathrm{CO}_{2} \\
\text { Fred, Peterson and Anderson, } \\
\quad 1923\end{array}$ \\
\hline $\begin{array}{l}\text { B. welchii } \\
\text { glycerol }\end{array}$ & $\begin{array}{l}\text { acrolein } \\
\text { Humphreys, } 19^{2} 4\end{array}$ \\
\hline $\begin{array}{l}\text { B. xylinum } \\
\text { (See Acetobacter xylinum) } \\
\text { (See also Bact. xylinum) } \\
\text { arabinose, dextrose, glycerol, } \\
\text { levulose, rhamnose } \\
\text { dextrose } \\
\text { glycerol } \\
\text { glycerol } \\
\text { glycerol } \\
\text { glycerol }\end{array}$ & $\begin{array}{l}\text { oxalic ac. } \\
\text { Banning, I902 } \\
\text { gluconic ac., ketogluconic ac. } \\
\text { Bernhauer and Schön, I929 } \\
\text { dihydroxyacetone } \\
\text { Bertrand and Sazerac, I90 I } \\
\text { dihydroxyacetone } \\
\text { Brit. patent } 269,950 \\
\text { dihydroxyacetone } \\
\text { Virtanen and Barlund, I926 } \\
\text { dihydroxyacetone } \\
\text { Bernhauer and Schön, I928 }\end{array}$ \\
\hline
\end{tabular}




Microorganisms
Substrates

B. xylinum (cont.) $\alpha$-ketoglutaric ac.

mannitol

methylethylacetaldehyde

sorbitol

sorbitol

sorbitol

\section{Bact. aceti}

(See also Mycoderma aceti) dextrose

ethyl alc.

propyl alc.

Bact. acidi propionici var. fuscum

(See also Propionibacterium Freudenreichii)

(See also Bact. acidi propionici a)

Bact. acidi propionici a

(See also Bact. acidi propionici var. fuscum)

(See also Propionibacterium Freudenreichii)

Bact. acidi propionici var. fuscum dextrose, lactic ac., lactose, pyruvic ac.

\section{Products}

Authors

succinic ac.

Iwatsuru, I925

levulose

Hoyer, I 898

i-amyl alc., i-valeric ac.

Neuberg and Simon, 1926

sorbose

Seifert, I 897

sorbose

Hoyer, $189^{8}$

sorbose

Mayer, 1898

gluconic ac.

Brown, i 886

acetic ac.

Brown, I 886

propionic ac.

Brown, 1886

acetic ac., propionic ac., succinic ac.

Virtanen, I923 
Microorganisms

Substrates

Bact. acidi propionici a lactic ac.

Bact. acidi propionici b

(See also Propionibacterium Fensenii)

Bact. acidi propionici b lactic ac.

Bact. acidi propionici c

(See Propionibacterium

$$
\text { Peterssonii) }
$$

Bact. acidi propionici d

(See also Propionibacterium Shermanii)

Bact. acidi propionici d lactose

Bact. acidi propionici var. rubrum (See Propionibacterium rubrum) (See Propionibacterium Thonii)

Bact. aerogenes

(See also B. lactis aerogenes)

Bact. aerogenes

glycerol

\section{Products}

Authors

acetic ac., propionic ac., $\mathrm{CO}_{2}$

von Freudenreich and Jensen, I 906

acetic ac., $\mathrm{CO}_{2}$, propionic ac.

von Freudenreich and Jensen, I 906

acetic ac., propionic ac.

Sherman and Shaw, 1923

acetic ac., 2-3 butylene glycol, $\mathrm{CO}_{2}$, ethyl alc., formic ac., $\mathrm{H}_{2}$, lactic ac., succinic ac. Braak, 1928 


Microorganisms
Substrates

Bact. ascendens

acetaldol

phenylglyoxal

Bact. caucasicum

lactose

Bact. cellaresolvens cellulose

Bact. cloacae

(See B. cloacae)

Bact. coli

(See also B. coli)

(See also B. coli communis)

(See also Bact. coli communis)

\section{Bact. coli}

galactose, lactose, levulose, malic ac., maltose, sucrose, tartaric ac.

glycerol

methyl glyoxal

pyruvic ac.

\section{Products}

Authors

$\beta$-butylene glycol, $\beta$-oxybutyric ac.

Binder-Kotrba, I926

d-mandelic ac.

Mayer, 1926

lactic ac., methyl glyoxal, pyruvic ac.

Kosty tschew and Soldatenkov, I 927

acetic ac., butyric ac., cellobiose lactic ac.

Groenewege, 1923

acetaldehyde

$$
\text { Nagai, } 1923
$$

acetic ac., $\mathrm{CO}_{2}$, ethyl alc., formic ac., $\mathrm{H}_{2}$, lactic ac., succinic ac.

Braak, 1928

lactic ac.

Neuberg and Gorr, 1925 acetic ac., $\mathrm{CO}_{2}$, formic ac., glycollic ac., $\mathrm{H}_{2}$, i-lactic ac., propionic ac.

Aubel, 1924 


Microorganisms
Substrates

Bact. dysenteriae Shiga-Kruse (See B. dysenteriae)

\section{Bact. Freundii glycerol}

Bact. gluconium dextrose gluconic ac. levulose

\section{Bact. lactis acidi}

dextrose, lactose, maltose

Bact. lactis aerogenes

(See also B. lactis aerogenes)

Bact. lactis aerogenes methylglyoxal

phenylglyoxal
Bact. opalescens cellulose

\section{Bact. pasteurianum}

(See also under Acetobacter)

\section{Products}

Authors

acetic ac., $\mathrm{CO}_{2}$, ethyl alc., formic ac., $\mathrm{H}_{2}$, lactic ac., trimethylene glycol, succinic ac.

Braak, I928

gluconic ac.

Hermann, I929

5-keto-gluconic ac.

Hermann, I929

5-keto-gluconic ac.

Hermann, I929

i-lactic ac.

Henneberg, I903

d, 1-lactic ac.

Neuberg and Simon, 1927

1-mandelic ac.

Neuberg and Simon, 1927

acetic ac., butyric ac., cellobiose, lactic ac.

Groenewege, 1923 
Microorganisms

Substrates

Bact. pasteurianum

d-l-methyl-ethyl acetaldehyde

methylglyoxal

\section{Bact. pyocyaneum}

(See B. pyocyaneus)

(See Bact. pyocyaneus)

Bact. pyocyaneus

(See also B. pyocyaneus)

Bact. pyocyaneus

dextrose

levulose

Bact. suipestifer citric ac.

Bact.termo

d-propylene glycol

tartaric ac.

Bact. thermoamylolyticum dextrose, sucrose

Bact.xylinum

(See also Acetobacter xylinum)

\section{Products}

Authors

d-amyl alc., valeric ac.

Neuberg and Simon, 1926

d-l-lactic ac.

Gorr and Perlmann, I 926

acetic ac., ethyl alc., formic ac.

Aubel, I92 I

acetic ac., ethyl alc., formic ac., lactic ac.

Aubel, 1921

acetic ac., $\mathrm{CO}_{2}$, succinic ac.

Brown, Duncan and Henry, $19^{2}+$

lactic ac., propionic ac.

LeBel, i 88 I

acetic ac., propionic ac., succinic ac.

König, I 88 I

acetic ac., butyric ac., $\mathrm{CO}_{2}, \mathrm{H}_{2}$, propionic ac.

Coolhaas, I928 


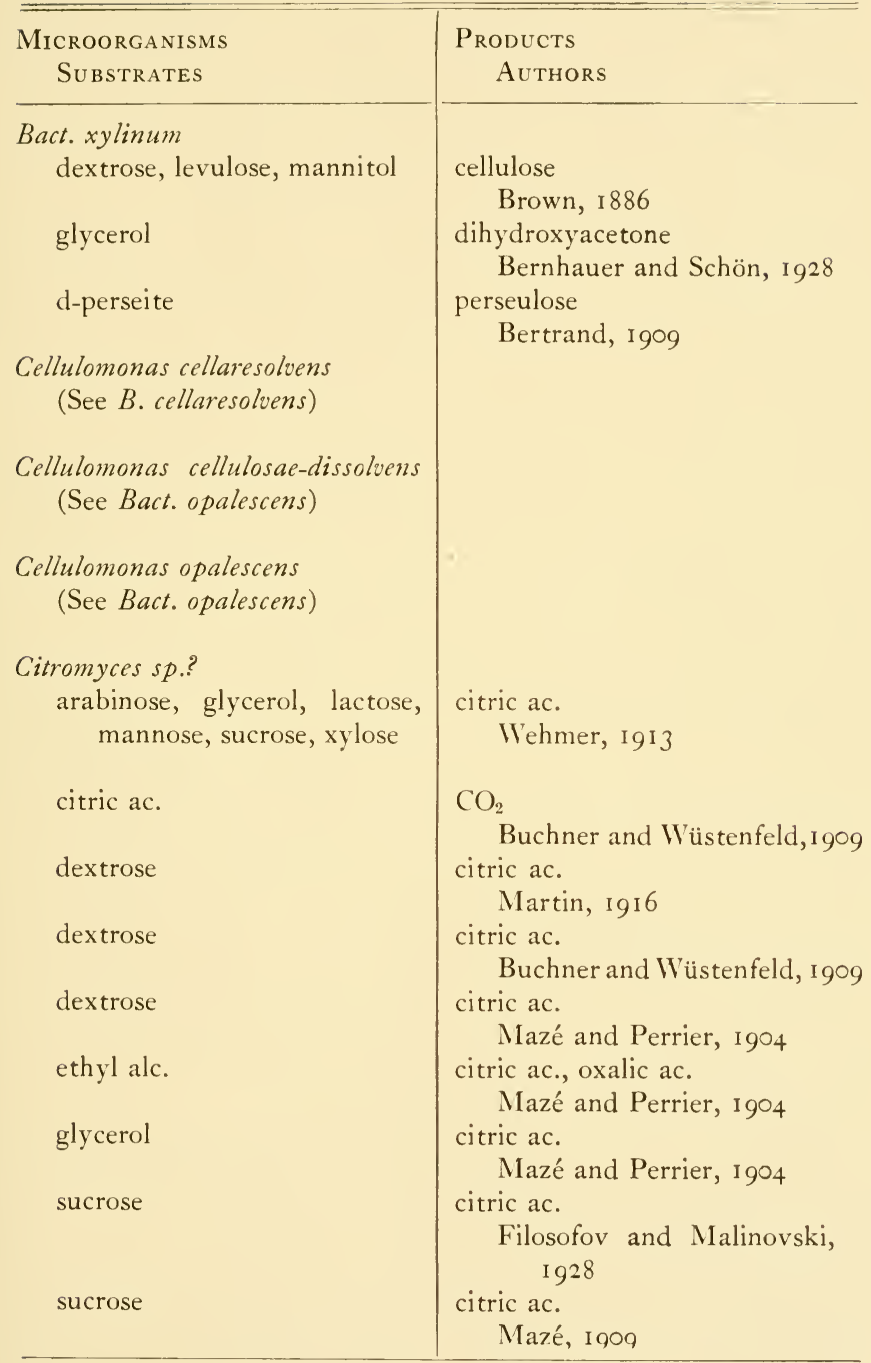




Microorganisms
Substrates
Citromyces sp.? (cont.)
sucrose
Citromyces glaber
arabinose, glycerol, mannitol,
sucrose
citric ac.
dextrose
quinic ac.
sucrose

Citromyces lacticus dextrose

Citromyces oxalicus dextrose

Citromyces Pfefferianus dextrose

sucrose

Citromyces tartricus dextrose ethyl alc.

Cladosporium, sp.? phloridzin

\section{Products}

Authors

citric ac., oxalic ac.,

Butkewitsch, 1922

citric ac., oxalic ac.

Butkewitsch, 1923 oxalic ac.

Butkewitsch, I 922 citric ac.

Butkewitsch, I922 protocatechuic ac., pyrocatechol

Butkewitsch, 1924 citric ac., gluconic ac.

Butkewitsch, 1927 citric ac., oxalic ac.

Butkewitsch, 1922

citric ac.

Mazé and Perrier, I904

citric ac.

Mazé and Perrier, 1904

citric ac.

Buchner and Wüstenfeld, I 909 citric ac., oxalic ac.

Butkewitsch, 1922

citric ac.

Mazé and Perrier, I904 citric ac., oxalic ac.

Mazé and Perrier, $190_{4}$

dextrose, phloroglucinol

Boas, I 9 I 6 


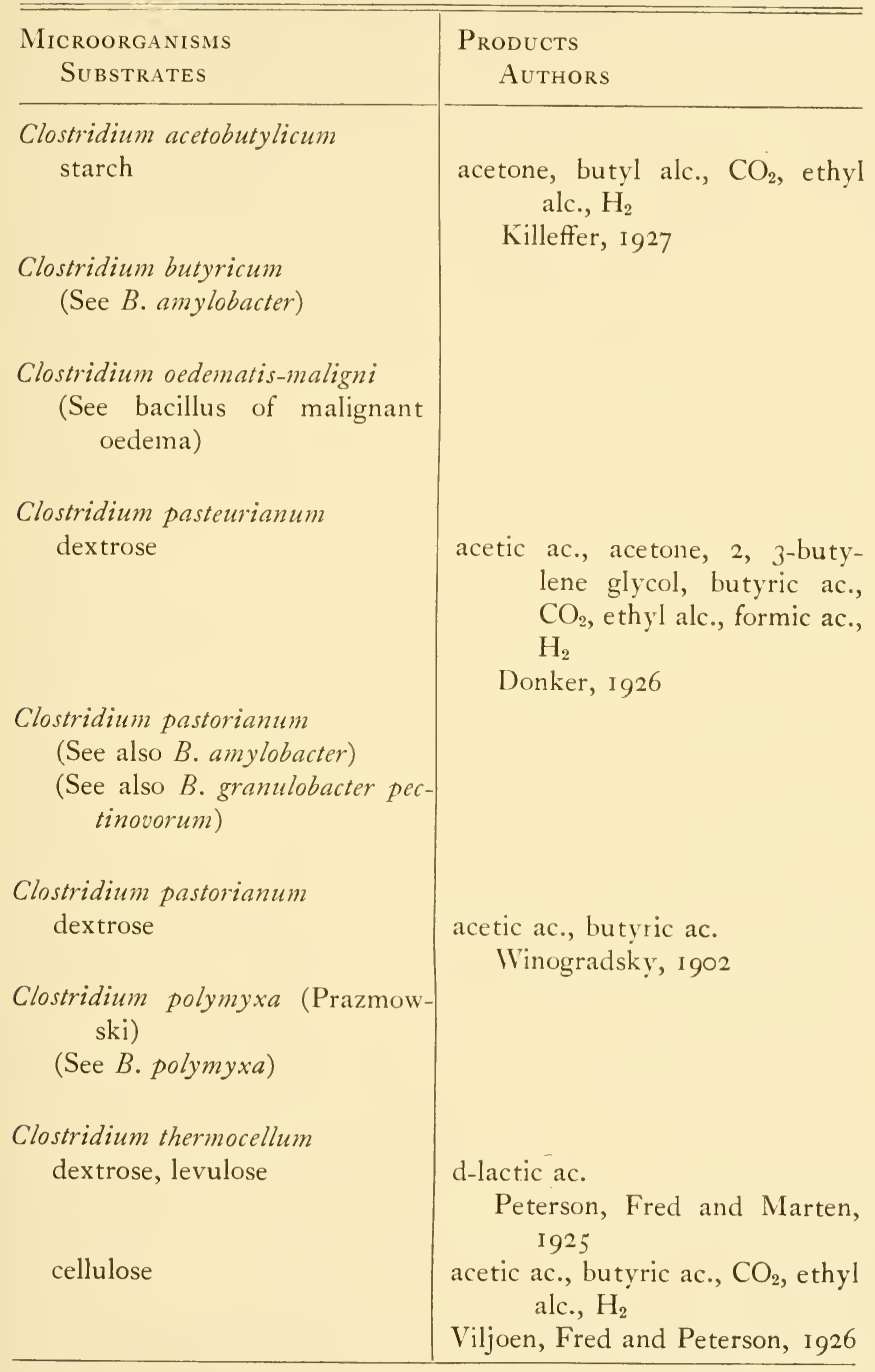




Microorganisms
Substrates

\section{Products}

Authors

Clostridium Welchii

(See B. Wielchii)

Diplococcus pneumoniae dextrose

Eberthella dysenteriae

(See B. dysenteriae)

Eberthella typhi

(See B.typhosus)

(See B. typhosum)

\section{Encapsulatus pneumoniae}

(See Friedländer's pneumobacillus)

Erythrobacillus prodigiosus

(See B. prodigiosus)

Escherichia coli

(See B. coli)

(See Bact. coli)

(See B. coli communis)

(See Bact. coli communis)

Friedländer's pneumococcus dextrose

mannitol

Fusarium lini dextrose acetic ac., propionic ac.

Brieger, 1883 acetic ac., ethyl alc., formic ac.

Frankland, Stanley and Frew, I 89 I

acetic ac., ethyl alc.

Frankland, Stanley and Frew, I 89 I

$\mathrm{CO}_{2}$, ethyl alc.

Anderson and Willaman, I922 
Microorganisms

Substrates
Products

Authors
Granulobacter butylicum

(See also B. amylobacter)

(See also Cl. butyricum)

Granulobacter saccharobutyricum dextrose

Granulobacterium butylicum starch

Klebsiella pneumoniae

(See Friedländer's pneumobacillus)

Lactobacillus, sp.?

dex trose

methylglyoxal

Lactobacillus arabinosus arabinose

Lactobacillus berolinensis

(See Saccharobacillus pastorianus var. berolinensis)

Lactobacillus casei

(See B. casei)

Lactobacillus caucasicus

(See Bact. caucasicum) acetic ac., 2, 3-butylene glycol, butyric ac., $\mathrm{CO}_{2}$, formic ac., $\mathrm{H}_{2}$, lactic ac.

Donker, I 926

ethyl alc., iso-butyl alc., n-butyl alc., iso-propyl alc., propyl alc.

Folpmers, I92 I

lactic ac.

Neuberg and Gorr, 1926 lactic ac.

Neuberg and Gorr, I926

acetic ac., lactic ac.

Fred, Peterson and Anderson, I 92 I 


\begin{tabular}{l} 
Microorganisms \\
Substrates \\
\hline $\begin{array}{l}\text { Lactobacillus Delbriicki } \\
\text { (See B. Delbriicki) }\end{array}$ \\
Lactobacillus leichmanni \\
dextrose \\
$\quad$ dextrose, galactose, lactose, \\
levulose
\end{tabular}

\section{Lactobacillus pentoaceticus}

arabinose, xylose

dextrose, galactose, lactic ac., mannose

levulose

malic ac., mannitol

pyruvic ac.

xylose

Lactobacillus pentosus

arabinose, xylose

Lactobacillus, sp.? phenylglyoxal

Liquidobacterium prodigiosum (See B. prodigiosus)

\section{Products}

Authors

1-lactic ac.

Allgeier and Peterson, I930 1-lactic ac.

Fred, Peterson and Stiles, 1925

acetic ac., lactic ac.

Fred, Peterson and Anderson, I 921

acetic ac., ethyl alc., lactic ac.

Peterson and Fred, I920 acetic ac., $\mathrm{CO}_{2}$, lactic ac., mannitol

Peterson and Fred, 1920 acetic ac., lactic ac.

Peterson and Fred, 1920 acetic ac., acetaldehyde, $\mathrm{CO}_{2}, \mathrm{H}_{2}$

Peterson and Fred, I920 acetic ac., i-lactic ac.

Fred, Peterson and Davenport, I9I9

acetic ac., lactic ac.

Fred, Peterson and Anderson, I92 I

1-mandelic ac.

Mayer, 1926 


\begin{tabular}{|c|c|}
\hline $\begin{array}{c}\text { Microorganisms } \\
\text { Substrates }\end{array}$ & $\begin{array}{l}\text { Products } \\
\text { Authors }\end{array}$ \\
\hline $\begin{array}{l}\text { Micrococcus casei liquefaciens } \\
\text { lactic ac., succinic ac. } \\
\text { lactic ac. }\end{array}$ & $\begin{array}{l}\text { acetic ac., } \\
\text { Orla-Jensen, I904 } \\
\text { acetic ac., propionic ac. } \\
\text { Orla-Jensen, I904 }\end{array}$ \\
\hline $\begin{array}{l}\text { Micrococcus climicus } \\
\text { quinic ac. }\end{array}$ & $\begin{array}{l}\text { protocatechuic ac. } \\
\text { Emmerling and Abderhalden, } \\
\text { I903 }\end{array}$ \\
\hline $\begin{array}{l}\text { Micrococcus oblongus } \\
\text { dextrose }\end{array}$ & $\begin{array}{l}\text { oxygluconic ac. } \\
\text { Boutroux, I } 886\end{array}$ \\
\hline $\begin{array}{l}\text { Monilia candida } \\
\text { dextrose } \\
\text { pyruvic ac. }\end{array}$ & $\begin{array}{l}\text { acetaldehyde } \\
\text { Cohen, I920 } \\
\text { acetaldehyde } \\
\text { Nagayama, I92 I }\end{array}$ \\
\hline $\begin{array}{l}\text { Mucor circinelloides (?) } \\
\text { dextrin, starch }\end{array}$ & $\begin{array}{l}\text { ethyl alc. } \\
\text { Gayon and Dubourg, I } 886\end{array}$ \\
\hline $\begin{array}{l}\text { Mucor mucedo } \\
\text { dextrose, sucrose }\end{array}$ & $\begin{array}{l}\text { ethyl alc. } \\
\text { Kostytschew and Eliasberg, } \\
\text { I } 920 \\
\text { ethyl alc., succinic ac. } \\
\text { Fitz, I } 873\end{array}$ \\
\hline $\begin{array}{l}\text { Mucor piriformis } \\
\text { dextrose }\end{array}$ & $\begin{array}{l}\text { citric ac. } \\
\text { Wehmer, I } 897\end{array}$ \\
\hline $\begin{array}{l}\text { Mucor plumbens } \\
\text { pyruvic ac. }\end{array}$ & $\begin{array}{l}\text { acetaldehyde } \\
\text { Nagayama, I92 I }\end{array}$ \\
\hline $\begin{array}{l}\text { Mucor racemosus } \\
\text { dextrose }\end{array}$ & $\begin{array}{l}\text { acetaldehyde } \\
\text { Cohen, I } 920\end{array}$ \\
\hline
\end{tabular}




Microorganisms
Substrates

Mucor racemosus (cont.) dextrose, sucrose

glycerol

pyruvic ac.

sucrose

sucrose

\section{Mucor Rouxii dextrose}

pyruvic ac.

\section{Mucor stolonifer}

(See also Rhizopus nigricans)

\section{Mucor stolonifer}

dex trose, sucrose

Mycoderma aceti

(See also Bact. aceti)

Mycoderma aceti dextrose

d- l-propylene glycol

2, 3-butylene glycol

\section{Products}

Authors

ethyl alc.

Kostytschew and Eliasberg, I 920

butyl alc., butyric ac., caproic ac., $\mathrm{CO}_{2}$, ethyl alc., $\mathrm{H}_{2}$

Fitz, I 876

acetaldehyde

Nagayama, I92 I

ethyl alc.

Fitz, 1876

ethyl alc., glycerol, succinic ac.

Emmerling, 1897

acetaldehyde

Cohen, 1920

acetaldehyde

Nagayama, I92 I

ethyl alc.

Kostytschew and Eliasberg, I 920

gluconic ac.

Boutroux, I 880

acetylcarbinol

Kling, I 901

acetylmethyl carbinol

Kling, I905 


\begin{tabular}{l} 
Microorganisms \\
Substrates \\
\hline
\end{tabular}

Mycoderma sp.? dextrose

Oidium albicans

lactic ac.

\section{Oidium farinosum}

lactic ac.

\section{Oidium gueraldi}

lactic ac.

Oidium lactis

dextrose

pyruvic ac.

Oidium lupuli

dextrose

\section{Oidium tenuis}

lactic ac.

Pectinobacter amylophilum starch

\section{Penicillum glaucum}

oleic ac.

sucrose

sucrose

sucrose

\section{Products}

Authors

pyruvic ac.

Fernbach and Schoen, I9I4

pyruvic ac.

Mazé and Ruot, I9I7

pyruvic ac.

Mazé and Ruot, I9I7

pyruvic ac.

Mazé and Ruot, 1917

acetaldehyde

Cohen, 1920

acetaldehyde

Nagayama, 192 I

acetaldehyde, acetic ac., ethyl alc., succinic ac.

Sumiki, I 927

pyruvic ac.

Mazé and Ruot, I9I7

acetic ac., butyl alc., formic ac., lactic ac., succinic ac.

Makrinov, I9I 5

keto-stearic ac.

Pigulewski and Charik, 1928 citric ac., gluconic ac.

Butkewitsch, 1927 ethyl alc.

Kosty tschew and A fanassjewa,

$$
\text { I } 922
$$

citric ac., oxalic ac.

Butkewitsch, 1922 


Microorganisms
Substrates

Penicillium luteum

dextrose

Penicillium purpurogenum dextrose

dextrose

Pneumobacillus of Friedländer arabinose

dulcitol

mannitol

xylose

Propionibacterium Freudenreichii (See also Bact. acidi propionici a)

(See also Bact. acidi propionici var. fuscum)

Propionibacterium Freudenreichii dextrose, glycerol, lactic ac.

Propionibacterium Fensenii

(See also Bact.acidi propionicib)

Propionibacterium Fensenii

dextrose, glycerol, lactic ac., pyruvic ac.

Propionibacterium pentosaceum

(See Bacillus acidi propionici)
Products

Authors

citric ac.

Wehmer, 1897

gluconic ac.

May, Thom and Church, 1927 gluconic ac.

Herrick and May, 1928

acetic ac., 1-lactic ac.

Grimbert, I 896 acetic ac., ethyl alc., succinic ac.

Grimbert, 1896 acetic ac., ethyl alc., succinic ac.

Grimbert, i 896 acetic ac., ethyl alc., 1-lactic ac., succinic ac.

Grimbert, I 896

acetic ac., $\mathrm{CO}_{2}$, propionic ac, succinic ac. van Niel, I928

acetic ac., $\mathrm{CO}_{2}$, propionic ac., succinic ac.

van Niel, 1928 
Microorganisms

Substrates

Propionibacterium pentosaceum arabinose, dextrose, glycerol, lactic ac., starch, xylose

dextrose, glycerol, lactic ac.

\section{Propionibacterium Peterssonii}

(See also Bact. acidi propionici c)

\section{Propionibacterium Peterssonii} dextrose, glycerol, lactic ac.

Propionibacterium rubrum

(See also Bact. acidi propionici var. rubrum)

Propionibacterium rubrum dextrose, lactic ac.

Propionibacterium Shermanii

(See also Bact. acidi propionici d)

Propionibacterium Shermanii dextrose, glycerol, lactic ac.

\section{Proprionibacterium technicum}

dextrose, glycerol, lactic ac., pyruvic ac., starch

Propionibacterium Thönii,

(See also Bact. acidi propionici var. rubrum)

\section{Products}

Authors

acetic ac., $\mathrm{CO}_{2}$, propionic ac.

Werkman, Hixon, Fulmer and Rayburn, I 929

acetic ac., $\mathrm{CO}_{2}$, propionic ac., succinic ac.

van Niel, 1928

acetic ac., $\mathrm{CO}_{2}$, propionic ac., succinic ac.

van Niel, I928

acetic ac., $\mathrm{CO}_{2}$, propionic ac., succinic ac.

van Niel, 1928

acetic ac., $\mathrm{CO}_{2}$, propionic ac., succinic ac.

van Niel, 1928

acetic ac., $\mathrm{CO}_{2}$, propionic ac., succinic ac.

van Niel, I 928 
Microorganisms

Substrates

Propionibacterium Thönii

dextrose, glycerol, lactic ac.

Pseudomonas aeruginosa

(See also B. pyocyaneus)

dextrose

Rhizopus, sp.?

dextrose

glycerol

Rhizopus chinensis

dextrose

Rhizopus nigricans

(See also Mucor stolonifer)

Rhizopus nigricans

dextrose, levulose

pyruvic ac.

sucrose, (invert)

Saccharobacillus pastorianus var. berolinensis

dextrin, sucrose

Saccharomyces cerevisiae

(See also under yeast)
Products

Authors

acetic ac., $\mathrm{CO}_{2}$, propionic ac., succinic ac.

- van Niel, I928

acetaldehyde, ethyl alc.

Nill, 1927

ethyl alc.

Takahashi and Sakaguchi, 1927

succinic ac.

Takahashi and Sakaguchi, I 927

1-lactic ac.

Saito, I9II

fumaric ac.

Ehrlich, I912

acetic ac., fumaric ac., lactic ac.

Gottschalk, 1925

fumaric ac., oxalic ac.

Butkewitsch, 1927

i-lactic ac.

Henneberg, I903 


\begin{tabular}{|c|c|}
\hline $\begin{array}{l}\text { Microorganisms } \\
\text { Substrates }\end{array}$ & $\begin{array}{l}\text { Products } \\
\text { Authors }\end{array}$ \\
\hline $\begin{array}{l}\text { Sacc. cerevisiae } \\
\text { arabinose }\end{array}$ & $\begin{array}{l}\mathrm{CO}_{2} \text {, ethyl alc., glyceric alde- } \\
\text { hyde (?) } \\
\text { Abbot, I926 } \\
\mathrm{CO}_{2} \text {, ethyl alc., glyceric alde- } \\
\text { hyde (?) } \\
\text { Abbot, I926 }\end{array}$ \\
\hline $\begin{array}{l}\text { Sacc. ellipsoideus } \\
\quad \text { sucrose }\end{array}$ & $\begin{array}{l}\text { glycerol } \\
\text { Adams, I919 }\end{array}$ \\
\hline $\begin{array}{l}\text { Sacc. Ludwigii } \\
\text { dextrose } \\
\text { dihydroxyace tone }\end{array}$ & $\begin{array}{l}\text { glycogen, } \mathrm{CO}_{2} \\
\text { Gottschalk, I925 } \\
\text { dextrose } \\
\quad \text { Neuberg and Kobel, I } 928\end{array}$ \\
\hline $\begin{array}{l}\text { Sacc. Saké } \\
\text { starch } \\
\text { sucrose }\end{array}$ & $\begin{array}{l}\text { dihexosan, amylobiose } \\
\text { Sjöberg, I } 927 \\
\text { acetaldehyde, acetic ac., ethyl } \\
\text { alc., } \mathrm{CO}_{2} \text {, glycerol } \\
\text { Kumagawa, } 1922\end{array}$ \\
\hline $\begin{array}{l}\text { Salmonella schottmiulleri } \\
\quad(\text { See B. paratyphoid B) }\end{array}$ & \\
\hline $\begin{array}{l}\text { Salmonella suipestifer } \\
\quad \text { (See Bact. suipestifer) }\end{array}$ & \\
\hline $\begin{array}{l}\text { Sarcina, sp.? } \\
\text { starch }\end{array}$ & $\begin{array}{l}\text { dextrin } \\
\text { Glimm and Grimm, I } 928\end{array}$ \\
\hline $\begin{array}{l}\text { Sarcina maxima } \\
\text { levulose }\end{array}$ & $\begin{array}{l}\text { acetic ac., butyric ac., } \mathrm{CO}_{2} \text {, ethyl } \\
\text { alc., formic ac., } \mathrm{H}_{2} \text {, lactic } \\
\text { ac., succinic ac. } \\
\text { Smit, I } 928\end{array}$ \\
\hline
\end{tabular}


levulose

Schizomycetes, sp. (?)

lactic ac.

quinic ac.

\section{Serratia marcescens}

(See also B. prodigiosus)

\section{Serratia marcescens}

dextrose

Sorbose bacterium

(See also Acetobacter sorbose)

Sorbose bacterium

arabinose

arabitol

dextrose

erythritol

\section{Products}

Authors

acetic ac., acetylmethyl carbinol, $\mathrm{CO}_{2}$, ethyl alc., formic ac., $\mathrm{H}_{2}$, lactic ac.

Smit, 1928

acetic ac., acetylmethyl carbinol, $\mathrm{CO}_{2}$, ethyl alc., $\mathrm{H}_{2}$, lactic ac.

Smit, 1928

propionic ac., valeric ac.

Fitz, I 880

acetic ac., formic ac., propionic ac., protocatechuic ac. Loew, I 88 I

acetic ac., acetylmethyl carbinol, 2, 3-butylene glycol, $\mathrm{CO}_{2}$, ethyl alc., formic ac., $\mathrm{H}_{2}$, lactic ac., succinic ac.

Pederson and Breed, 1928

arabonic ac.

Bertrand, I 898

keto-sugar

Bertrand, I 898

gluconic ac.

Bertrand, 1898

ery thrulose

Bertrand, I 898,1900 


\begin{tabular}{|c|c|}
\hline $\begin{array}{l}\text { Microorganisms } \\
\text { Substrates }\end{array}$ & $\begin{array}{l}\text { Products } \\
\text { AUTHORS }\end{array}$ \\
\hline \multicolumn{2}{|l|}{ Sorbose bacterium (cont.) } \\
\hline galactose & $\begin{array}{l}\text { galactonic ac. } \\
\text { Bertrand, I } 898\end{array}$ \\
\hline a-glucoheptite & $\begin{array}{l}\text { a-glucoheptulose } \\
\text { Bertrand and Nitzberg, I928 }\end{array}$ \\
\hline glycerol & $\begin{array}{l}\text { dihydroxyacetone } \\
\text { Bertrand, } 1898\end{array}$ \\
\hline d-mannitol & $\begin{array}{l}\text { levulose } \\
\text { Bertrand, I } 898\end{array}$ \\
\hline perseitol & $\begin{array}{l}\text { keto-sugar } \\
\quad \text { Bertrand, } 1898\end{array}$ \\
\hline d-sorbitol & $\begin{array}{l}\text { sorbose } \\
\quad \text { Bertrand, I } 898\end{array}$ \\
\hline valemite & $\begin{array}{l}\text { keto-sugar } \\
\quad \text { Bertrand, I } 898\end{array}$ \\
\hline xylose & $\begin{array}{l}\text { xylonic ac. } \\
\text { Bertrand, } 189^{8}\end{array}$ \\
\hline \multicolumn{2}{|l|}{ Sterigmatocystis nigra } \\
\hline sucrose & $\begin{array}{l}\text { citric ac., gluconic ac., oxalic ac. } \\
\text { Molliard, I922 }\end{array}$ \\
\hline \multicolumn{2}{|l|}{ Streptococcus brevis } \\
\hline dextrose & $\begin{array}{c}\text { acetic ac., lactic ac. } \\
\text { Hucker, } 1928\end{array}$ \\
\hline \multicolumn{2}{|l|}{ Streptococcus cremoris } \\
\hline dex trose & $\begin{array}{l}\text { acetic ac., lactic ac. } \\
\text { Hucker, I928 }\end{array}$ \\
\hline \multicolumn{2}{|l|}{ Streptococcus fecalis } \\
\hline dextrose & $\begin{array}{l}\text { acetic ac., lactic ac. } \\
\text { Hucker, } 1928\end{array}$ \\
\hline \multicolumn{2}{|l|}{ Streptococcus hemolyticus } \\
\hline dextrose & $\begin{array}{c}\text { acetic ac., lactic ac. } \\
\text { Hucker, I928 }\end{array}$ \\
\hline $\begin{array}{l}\text { Streptococcus hornensis } \\
\quad \text { sucrose }\end{array}$ & $\begin{array}{l}\text { dextrose } \\
\quad \text { Boekhout, I } 900\end{array}$ \\
\hline
\end{tabular}




Microorganisms
Substrates

Streptococcus inulinareous dextrose

Streptococcus lactis dextrose

dextrose

sucrose

Streptococcus mitior dextrose

Streptococcus paracitrovorous dextrose

Streptococcus pyogenes dextrose

Streptococcus stenos dextrose

Streptococcus viridans dextrose

Termobactcrium aceti acetic ac., arabinose, dex trose, ethylene glycol, galactose, glycerol, glycollic ac., lactic ac., malonic ac.

Thermobacillus tarbellicus sucrose

Tyrothrix tenuis

dex trose, glycerol, mannitol
Products

AUTHORS

acetic ac., lactic ac.

Hucker, 1928

d-lactic ac.

Allgeier and Peterson, I930 acetic ac., lactic ac.

Hucker, 1928

d-lactic ac.

Virtanen, Wichmann and Lindström, 1927

acetic ac., lactic ac.

Hucker, 1928

acetic ac., lactic ac.

Hucker, 1928

acetic ac., lactic ac.

Hucker, 1928

acetic ac., lactic ac.

Hucker, 1928

acetic ac., lactic ac.

Hucker, 1928

oxalic ac.

Banning, 1902

lactic ac.

Guittonneau, 1928

acetylmethyl carbinol Harden and Norris, I 9 I 2 


\begin{tabular}{|c|c|}
\hline $\begin{array}{l}\text { Microorganisms } \\
\text { Substrates }\end{array}$ & $\begin{array}{l}\text { Products } \\
\text { Authors }\end{array}$ \\
\hline \multicolumn{2}{|l|}{$\begin{array}{l}\text { Yeast } \\
\qquad \text { (See also Sacc. cerevisiae) }\end{array}$} \\
\hline \multicolumn{2}{|l|}{ Yeast } \\
\hline acetaldehyde & $\begin{array}{l}\text { ethyl alc. } \\
\text { Kostytschew and Hübbenet, } \\
\text { I9I } 2\end{array}$ \\
\hline acetaldehyde & $\begin{array}{l}\text { ethyl alc., acetic ac. } \\
\text { Kostytschew, I9I } 4\end{array}$ \\
\hline acetaldehyde, sucrose & $\begin{array}{l}\text { acetoin } \\
\quad \text { Neuberg and Simon, I925 }\end{array}$ \\
\hline acetaldol & $\begin{array}{l}\beta \text {-butyleneglycol } \\
\text { Neuberg and Kerb, I9I } 8\end{array}$ \\
\hline acetophenone, sucrose & $\begin{array}{l}\text { l-phenylmethyl carbinol } \\
\text { Neuberg and Nord, I919 }\end{array}$ \\
\hline acetylmethyl carbinol & $\begin{array}{l}\text { 2, 3-butylene glycol } \\
\text { Neuberg and Kobel, } 1925\end{array}$ \\
\hline aldehydropropionic ac. & $\begin{array}{l}\text { succinic ac. } \\
\text { Neuberg and Ringer, I91 } 8\end{array}$ \\
\hline benzaldehyde & $\begin{array}{l}\text { benzyl alc. } \\
\text { Neuberg and Welde, I9It }\end{array}$ \\
\hline benzaldehyde & $\begin{array}{l}\text { l-phenylacetyl carbinol } \\
\text { Neuberg and Ohle, I922 }\end{array}$ \\
\hline benzil & $\begin{array}{l}\text { benzoin } \\
\text { Neuberg and Nord, I9I9 }\end{array}$ \\
\hline benzylpyruvic ac. & $\begin{array}{l}\text { phenylpropylaldehyde } \\
\text { Rona, I9I4 }\end{array}$ \\
\hline isobutylaldehyde & $\begin{array}{l}\text { isobutyl alc. } \\
\text { Ohta, I } 9^{1} 4\end{array}$ \\
\hline caproicaldehyde & $\begin{array}{l}\text { n-hexyl alc. } \\
\text { Neuberg and Nord, I9I4 }\end{array}$ \\
\hline chlorobenzaldehyde & $\begin{array}{l}\text { acetyl-chlorbenzyl carbinol, p- } \\
\text { benzyl alc., chlorobenzoic } \\
\text { ac., chlorobenzyl alc. }\end{array}$ \\
\hline & $\begin{array}{c}\text { Neuberg and Liebermann, } \\
\text { I92I }\end{array}$ \\
\hline
\end{tabular}




\begin{tabular}{|c|c|}
\hline $\begin{array}{l}\text { Microorganisms } \\
\text { Substrates }\end{array}$ & $\begin{array}{l}\text { Products } \\
\text { Authors }\end{array}$ \\
\hline \\
\hline cinnamic aldehyde & $\begin{array}{l}\text { cinnamic alc. } \\
\text { Rona, I9I4 }\end{array}$ \\
\hline citral & $\begin{array}{l}\text { geraniol } \\
\quad \text { Neuberg and Kerb, I9I } 8\end{array}$ \\
\hline d-citronellaldehyde & $\begin{array}{l}\text { citronella oil or alc. } \\
\text { Mayer and Neuberg, I9I } 5\end{array}$ \\
\hline dextrose & $\begin{array}{l}\text { acetaldehyde } \\
\text { Kostytschew, I } 912\end{array}$ \\
\hline dextrose, levulose & $\begin{array}{l}\text { acetylmethyl carbinol, 2, 3-buty- } \\
\text { lene glycol }\end{array}$ \\
\hline & $\begin{array}{l}\text { Kluyver, Donker and Hooft, } \\
\text { I } 925\end{array}$ \\
\hline $\begin{array}{l}\text { dextrose, dioxyacetone, gly- } \\
\text { ceraldehyde }\end{array}$ & $\begin{array}{l}\text { lactic ac. } \\
\text { Oppenheimer, I9I3 }\end{array}$ \\
\hline dex trose & $\begin{array}{l}\text { glycerol } \\
\text { Oppenheimer, I913 }\end{array}$ \\
\hline dextrose & $\begin{array}{l}\text { lactic ac. } \\
\text { Aubel, I } 929\end{array}$ \\
\hline dex trose & $\begin{array}{l}\text { pyruvic ac. } \\
\text { Fernbach and Schoen, } 1920\end{array}$ \\
\hline dextrose, sucrose & $\begin{array}{l}\text { acetaldehyde, acetic ac., ethyl } \\
\text { alc., glycerol } \\
\text { Neuberg and Hirsch, I9I9 }\end{array}$ \\
\hline dextrose with brucine & $\begin{array}{l}\text { brucine pyruvate } \\
\text { Traetta-Mosca, } 1927\end{array}$ \\
\hline a. a. dichloracetone & $\begin{array}{l}\text { a. a. dichlorpropyl alc. } \\
\text { Sen, } 19^{2} 4\end{array}$ \\
\hline dihydroxymaleic ac. & $\begin{array}{l}\mathrm{CO}_{2} \text {, glycollicaldehyde } \\
\text { Neuberg and Schwenk, I9I } 5\end{array}$ \\
\hline enanthol & $\begin{array}{l}\text { n-heptyl alc. } \\
\text { Ohta, I9I4 }\end{array}$ \\
\hline ethyl alc. & $\begin{array}{l}\text { acetaldehyde } \\
\text { Trillat and Sauton, I } 908\end{array}$ \\
\hline ethyl disulfide & $\begin{array}{l}\text { ethyl mercaptan } \\
\text { Neuberg and Schwenk, I9I } 5\end{array}$ \\
\hline
\end{tabular}




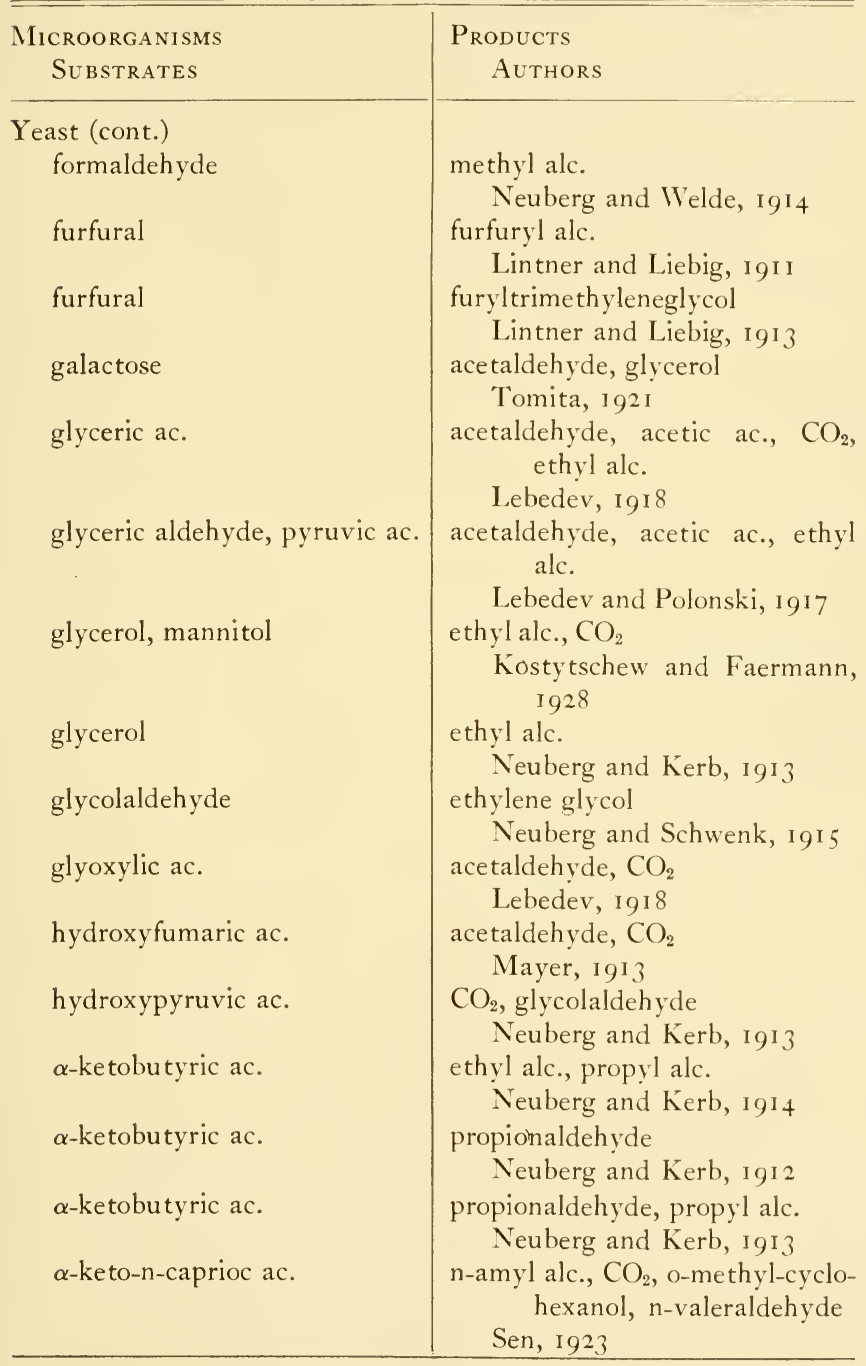


Microorganisms

Substrates

Yeast (cont.)

ketoglutaric ac.

$\alpha$-ketoisovaleric ac.

lactic ac.

lactose

levulose

malic ac.

mannose

methoxybenzaldehyde

methyl-benzoyl-carbinol+ dextrose

methylethyl ketone+sucrose

methylethyl pyruvic ac.

methyl- $\alpha$-chlorethyl ketone

methyl glyoxal

methyl glyoxal

methyl glyoxal
Products

Authors

$\mathrm{CO}_{2}$, succinic ac.

Neuberg and Ringer, I9I 4 isobutyl aldehyde

Sen, 1923

ethyl alc., pyruvic ac.

Kayser, 1923

acetaldehyde

Trillat, I 908

acetylmethyl carbinol, 2, 3-butylene glycol

Kluyver and Donker, 1924 lactic ac.

Lebedev and Russ, I9I6 ethyl alc.

Mezzadroli, I9 8 methoxybenzoic ac.

Neuberg and Liebermann, I 92 I

methyl-phenyl-ethylene glycol

Neuberg and Komarewsky, 1927

d-methylethyl carbinol

Neuberg and Nord, I9I 9 amyl alc., methyl-ethyl acetaldehyde, valeric ac.

Neuberg and Peterson, I 9 I 4 methyl- $\alpha$-chlorethyl alc.

Santomauro, 1924 d-lactic ac.

Neuberg and Kobel, 1927, I929

d-l-lactic ac.

Neuberg, 19I3

lactic ac.

Dakin and Dudley, I9I3 


\begin{tabular}{|c|c|}
\hline $\begin{array}{l}\text { Microorganisms } \\
\text { Substrates }\end{array}$ & $\begin{array}{l}\text { Products } \\
\text { Authors }\end{array}$ \\
\hline \multicolumn{2}{|l|}{ Yeast (cont.) } \\
\hline methyl-n-hexyl ketone & $\begin{array}{l}\text { d-methyl hexyl carbinol } \\
\text { Neuberg and Nord, I9I9 }\end{array}$ \\
\hline methylnonyl ketone & $\begin{array}{l}\text { d-methyl nonyl carbinol } \\
\text { Neuberg and Nord, I9I9 }\end{array}$ \\
\hline $\begin{array}{l}\text { methyl-n-propyl ketone }+ \\
\text { sucrose }\end{array}$ & $\begin{array}{l}\text { methyl-n-propyl carbinol } \\
\text { Neuberg and Nord, I919 }\end{array}$ \\
\hline oxalacetic ac. & $\begin{array}{l}\text { acetaldehyde, acetone, } \mathrm{CO}_{2} \\
\text { Neuberg and Gorr, } 1925\end{array}$ \\
\hline oxalacetic ac. & $\begin{array}{l}\text { acetaldehyde, } \mathrm{CO}_{2} \\
\text { Neuberg and Kerb, I9I3 }\end{array}$ \\
\hline oxalacetic ac. & $\begin{array}{c}\text { acetylmethyl carbinol, 2,3-buty- } \\
\text { lene glycol, malic ac. } \\
\text { Neuberg and Gorr, 1924 }\end{array}$ \\
\hline phenylacetaldehyde & $\begin{array}{l}\text { phenylethyl alc. } \\
\text { Neuberg and Welde, I9I4 }\end{array}$ \\
\hline phenylglyoxal & $\begin{array}{l}\text { mandelic ac. } \\
\text { Dakin and Dudley, I9I3 }\end{array}$ \\
\hline phenylglyoxalic ac. & $\begin{array}{l}\text { benzaldehyde } \\
\text { Binder-Kotrba, I926 }\end{array}$ \\
\hline pyruvic ac. & $\begin{array}{l}\text { acetaldehyde, } \mathrm{CO}_{2} \\
\text { Gottschalk, I } 923\end{array}$ \\
\hline pyruvic ac. & $\begin{array}{l}\text { acetaldehyde } \\
\text { Neuberg and Reinfürth, I } 920\end{array}$ \\
\hline pyruvic ac. & $\begin{array}{l}\text { acetylmethyl carbinol } \\
\text { Hirsch, } 1922\end{array}$ \\
\hline pyruvic ac. & $\begin{array}{l}\text { acetaldehyde, } \mathrm{CO}_{2} \\
\text { Neuberg and Kerb, I } 9{ }^{12}\end{array}$ \\
\hline pyruvic ac. & $\begin{array}{l}\text { acetylmethyl carbinol, } \mathrm{CO}_{2} \\
\text { Neuberg and May, } 9^{2} 3\end{array}$ \\
\hline pyruvic ac. & $\begin{array}{l}\text { aldol } \\
\text { Neuberg, I9 } 12\end{array}$ \\
\hline pyruvic ac. & $\begin{array}{l}\text { ethyl alc. } \\
\text { Neuberg and Kerb, } 1913\end{array}$ \\
\hline pyruvic ac., sucrose & $\begin{array}{l}\text { acetaldehyde } \\
\text { Paris, I } 922\end{array}$ \\
\hline
\end{tabular}




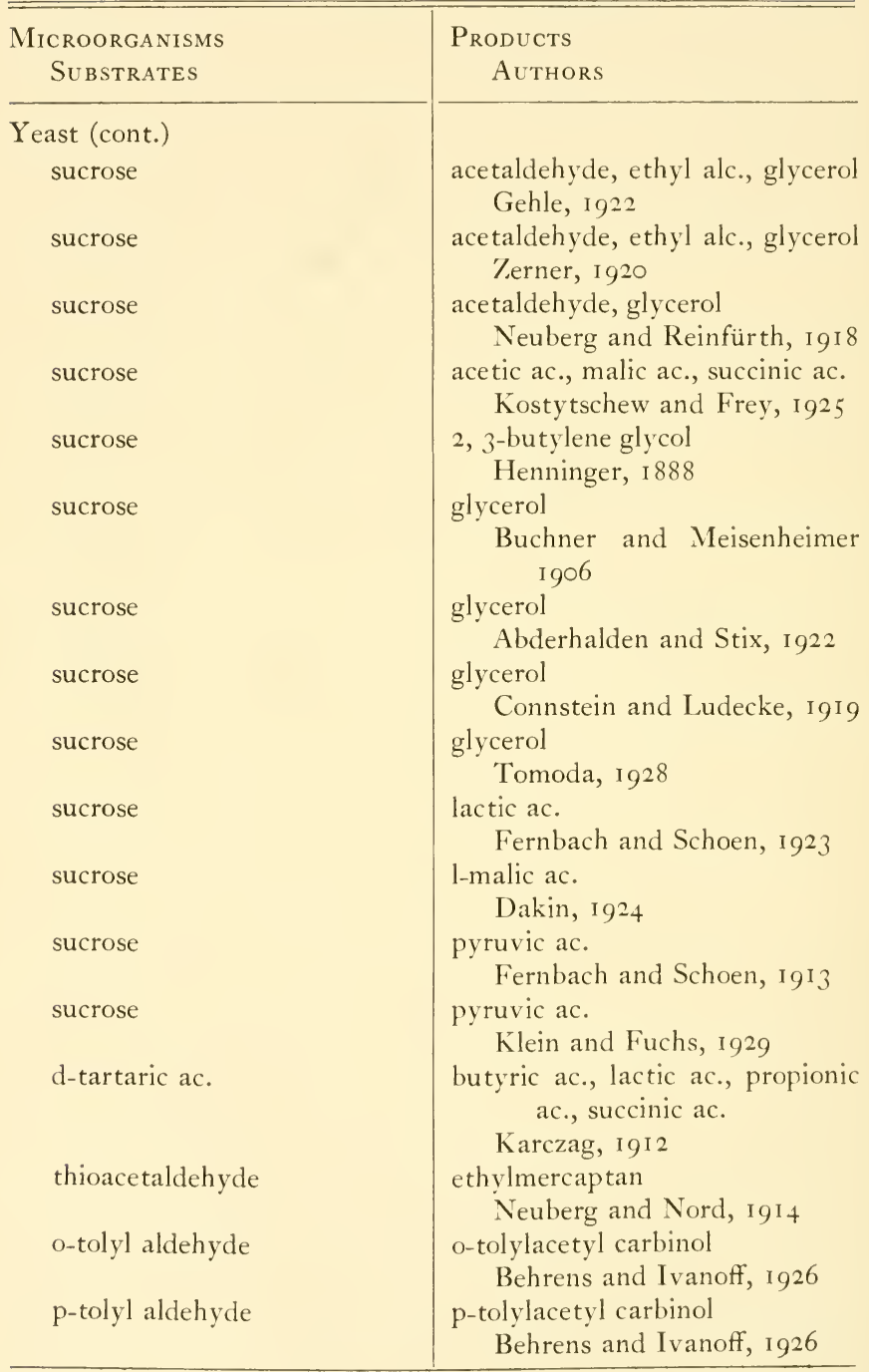




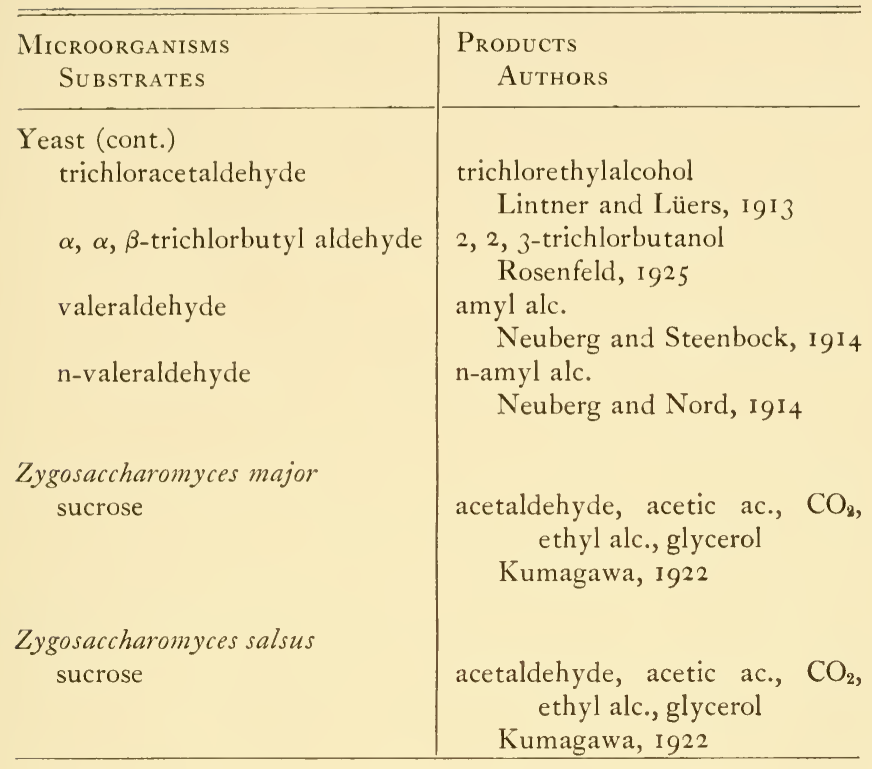



CHAPTER II

INDEX TO ORGANIC (NON-NITROGENOUS) SUBSTRATES 



\section{CHAPTER II}

TABLE TIVO

INDEX TO ORGANIC (NON-NITROGENOUS) SUBSTRATES

\begin{tabular}{|c|c|}
\hline $\begin{array}{l}\text { Substrates } \\
\text { Products }\end{array}$ & $\begin{array}{l}\text { Microorganisms } \\
\text { Authors }\end{array}$ \\
\hline \multicolumn{2}{|l|}{ ACETALDEHYDE } \\
\hline acetic ac. & $\begin{array}{l}\text { B. pyocyaneus } \\
\text { Supniewski, I } 923\end{array}$ \\
\hline acetylmethyl carbinol & $\begin{array}{l}\text { yeast } \\
\text { Neuberg and Simon, I925 }\end{array}$ \\
\hline acetic ac., ethyl alc. & $\begin{array}{l}\text { yeast } \\
\text { Kostytschew, I9 }{ }_{4}\end{array}$ \\
\hline $\begin{array}{l}\text { acetic ac., 2, 3-butylene-glycol, } \\
\text { succinic ac. }\end{array}$ & $\begin{array}{l}\text { B. lactis aerogenes } \\
\text { Harden and Norris, I9 I } 2\end{array}$ \\
\hline ethyl alc. & $\begin{array}{l}\text { yeast } \\
\text { Kostytschew and Hubbenet, } \\
\text { I9 } 2\end{array}$ \\
\hline \multicolumn{2}{|l|}{ ACETA LDOL } \\
\hline $\begin{array}{l}\beta \text {, butylene glycol, } \beta \text {, hydroxy- } \\
\text { butyric ac. }\end{array}$ & $\begin{array}{l}\text { Bact. ascendens } \\
\text { Binder-Kotrba, } 1926\end{array}$ \\
\hline$\beta$, butylene glycol & $\begin{array}{l}\text { yeast } \\
\text { Neuberg and Kerb, I9 } 18\end{array}$ \\
\hline \multicolumn{2}{|l|}{ Acetic Ac. } \\
\hline formaldehyde, formic ac. & $\begin{array}{l}\text { B. pyocyaneus } \\
\text { Supniewski, I } 923\end{array}$ \\
\hline glycollic ac. & $\begin{array}{l}\text { Asp. niger } \\
\text { Challenger, Subramaniam and } \\
\text { Walker, } 1927\end{array}$ \\
\hline oxalic ac. & $\begin{array}{l}\text { Asp. niger } \\
\text { Wehmer, I } 89 \mathrm{I}\end{array}$ \\
\hline
\end{tabular}


Substrates

Products

Acetic Ac. (cont.)

oxalic ac.

oxalic ac.

oxalic ac.

\section{Acetoin}

See acetylmethyl carbinol

\section{Acetone}

acetic ac., formic ac.

Acetonedicarboxylic ac. oxalic ac.

Acetophenone + sucrose

1-phenylmethyl carbinol

Acetylmethyl carbinol

2, 3-butylene glycol

Adonitol

acetylmethyl carbinol, 2, 3butylene glycol d-adoninulose

Aldehydropropionic ac. succinic ac.
Microorganisms

AUTHORS

Asp. niger

Challenger, Subramaniam and

Walker, I 927

Asp. niger

Raistrick and Clark, I9I9

B. industrium, B. oxydans, Termobacterium aceti

Banning, 1902

B. pyocyaneus

Supniewski, I 923

Asp. niger

Walker, Subramaniam and Challenger, 1927

yeast

Neuberg and Nord, I9I9

yeast

Neuberg and Kobel, 1925

B. lactis aerogenes

Harden and Norris, I91 2

Acetobacter suboxydans

Visser 't Hooft, 1925

yeast

Neuberg and Ringer, I9I 8 


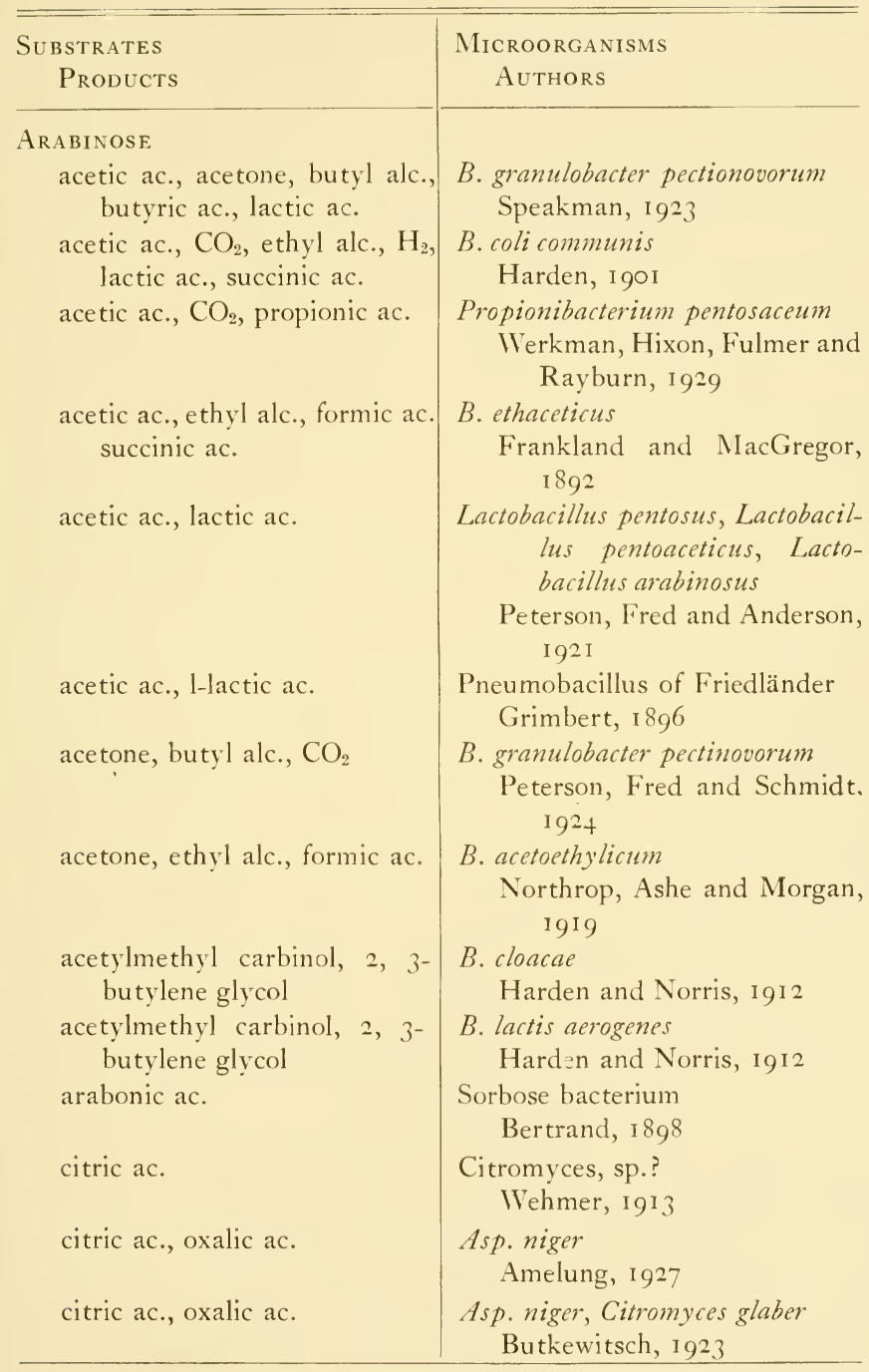


Substrates

Products

Arabinose (cont.)

$\mathrm{CO}_{2}$

$\mathrm{CO}_{2}$, ethyl alc., glyceric ald. (?)

fumaric ac., citric ac.

gluconic ac.

oxalic ac.

Arabitol

keto-pentose

BENZALDEHyde

benzyl alc.

phenyl-acetyl carbinol

BENZIL

benzoin

BENZYLPYRUVIC AC.

phenylpropyl aldehyde

Butyl alc.

butyric ac.

butyric ac.
Microorganisms

Authors

B. heribicola aureum

Fred, Peterson and Anderson, 1923

Sacc. cerevisiae

Abbott, 1926

Asp. fumaricus

Schreyer, I 928

Asp. niger

Bernhauer, 1928

$B$. acoti, B. acetigenum, B. acetosum, $B$. ascendens, $B$. Kïtzingianum, B.xylinum, Termobacterium aceti

Banning, 1902

sorbose bacterium

Bertrand, I 898

yeast

Neuberg and Welde, I9I4 yeast

Neuberg and Ohle, I922

yeast

Neuberg and Gorr, I9I9

yeast

Rona, I9I4

B. Kützingianum, B. Pasteurianum

Seifert, I 897

Acetobacter melanogenum

Visser't Hooft, I925 


\begin{tabular}{|c|c|}
\hline $\begin{array}{l}\text { Substrates } \\
\text { Products }\end{array}$ & $\begin{array}{c}\text { Microorganismis } \\
\text { Authors }\end{array}$ \\
\hline $\begin{array}{l}\text { 2, 3-BUTYLENE GLYCOL } \\
\text { acetone } \\
\text { acetylmethyl carbinol }\end{array}$ & $\begin{array}{l}\text { Acetobacter suboxydans } \\
\text { Acetobacter xylinum } \\
\text { Visser't Hooft, I } 925 \\
\text { Sorbose bacteria, Mycoderma acet } \\
\text { Kling, I } 905\end{array}$ \\
\hline $\begin{array}{l}\text { ButYRIC AC. } \\
\beta \text {-hydroxybutyric ac., aceto- } \\
\text { acetic ac., acetone }\end{array}$ & $\begin{array}{l}\text { Asp. niger } \\
\text { Coppock, Subramaniam and } \\
\text { Walker, } 1928\end{array}$ \\
\hline $\begin{array}{l}\text { CAPROICALDEHYDE } \\
\text { n-hexyl alc. }\end{array}$ & $\begin{array}{l}\text { yeast } \\
\text { Neuberg and Nord, I } 9^{I} 4\end{array}$ \\
\hline $\begin{array}{l}\text { CEllulose } \\
\text { acetic ac., butyric ac., } \mathrm{CO}_{2} \text {, } \\
\text { ethyl alc., } \mathrm{H}_{2} \\
\text { acetic ac., butyric ac., } \mathrm{CO}_{2}, \\
\text { ethyl alc., } \mathrm{H}_{2} \\
\text { acetic ac., butyric ac., lactic } \\
\text { ac., cellobiose }\end{array}$ & $\begin{array}{l}\text { Clostridium thermocellum } \\
\text { Viljoen, Fred and Peterson, } \\
\quad \text { I926 } \\
\text { B. cellulosae dissolvens } \\
\text { Khouvine, I } 923 \\
\text { Bact. cellaresolvens } \\
\text { Bact. opalescens } \\
\text { Groenewege, } 1923\end{array}$ \\
\hline $\begin{array}{l}\text { CHLORAL } \\
\text { (See trichloracetaldehyde) }\end{array}$ & \\
\hline $\begin{array}{l}\text { CH LOROBENZALDEHYDE } \\
\text { chlorobenzoic ac., chlorobenzyl } \\
\text { alc. }\end{array}$ & $\begin{array}{l}\text { yeast } \\
\text { Neuberg and Liebermann, } \\
1921\end{array}$ \\
\hline $\begin{array}{l}\text { Cinnamic ac. } \\
\text { styrol }\end{array}$ & $\begin{array}{l}\text { Asp. niger } \\
\text { Herzog and Ripke, I908 }\end{array}$ \\
\hline $\begin{array}{l}\text { CINNAMIC ALDEHYDE } \\
\text { cinnamic alc. }\end{array}$ & $\begin{array}{l}\text { yeast } \\
\text { Rona, I9I4 }\end{array}$ \\
\hline
\end{tabular}


Substrates

Products
Microorganisms

AUthors
Citral

geraniol

\section{Citric ac.}

acetic ac., $\mathrm{CO}_{2}$, succinic ac.

acetic ac., $\mathrm{CO}_{2}$, ethyl alc., succinic ac.

acetone, glyoxylic ac., malonic ac., oxalic ac.

acetonedicarboxylic ac.

glycollic ac.

oxalic ac.

oxalic ac.

\section{$\alpha$-Citronell aldehyde}

$\alpha$-citronella oil or alc.

\section{Dextrin}

acetone, ethyl alc., formic ac.

ethyl alc. yeast

Neuberg and Kerb, 1918

B. suipestifer

Brown, Duncan and Henry, 1924

B. coli communis

Grey, 1923

Asp. niger

Challenger, Subramaniam and Walker, I 927

Asp. niger

Challenger, Subramaniam and Walker, 1927

Asp. niger

Challenger, Subramaniam and Walker, 1927

Citromyces glaber

Butkewitsch, 1922

Asp. niger

Challenger, Subramaniam and Walker, 1927

yeast

Mayer and Neuberg, I9I 5

B. acetoethylicum

Northrop, Ashe and Morgan, I9I 9

Mucor circinelloides (?)

Gayon and Dubourg, I 886 
Substrates

Products

Dextrin (cont.)

oxalic ac.

oxalic ac.

\section{Dextrose}

acetaldehyde

acetaldehyde

acetaldehyde

acetaldehyde

acetaldehyde, acetic ac., ethyl alc., glycerol

acetaldehyde, acetic ac., ethyl alc., succinic ac.

acetaldehyde, acetylmethyl carbinol, 2, 3-butylene gly$\mathrm{col}$

acetaldehyde, acetic ac., ethyl alc., formic ac., succinic ac. acetic ac.

acetic ac., acetone, butyl alc., butyric ac., lactic ac.
Microorganisms

AUTHORs

Asp. niger

Elfving, I9I 9

B. acetosum

B. Kützingianum

B. Pasteurianum

Banning, I 902

B. coli communis

Grey, I9I3

Asp. cellulosae

Monilia candida

Mucor racemosus

Mucor rouxii

Oidium lactis

Cohen, 1920

$B$. lactis aerogenes

Kumagawa, 1922

yeast

Kosty tschew, 1912

yeast

Neuberg and Hirsch, I9I9

Oidium lupuli

Sumiki, I927

$B$. lactis aerogenes

Neuberg, Nord and Wolff, I 920

B. dysenteriae, Shiga-Kruse

Bergh, I928

B. coli

Scheffer, 1928

B. granulobacter pectinovorum

Speakman, I 923 


\section{Substrates}

Products
Microorganisms

Authors

\section{Dextrose (cont.)}

acetic ac., acetone, 2, 3-butylene glycol, butyric ac., $\mathrm{CO}_{2}$, ethyl alc., formic ac., $\mathrm{H}_{2}$

acetic ac., acetone, ethyl alc., formic ac., lactic ac.

acetic ac., acetylmethyl carbinol, acetone, butyl alc., 2, 3-butylene glycol, $\mathrm{CO}_{2}$, ethyl alc., formic ac., $\mathrm{H}_{2}$, lactic ac.

acetic ac., acetylmethyl carbinol, acetone, 2, 3-butylene glycol, $\mathrm{CO}_{2}$, ethyl alc., formic ac., $\mathrm{H}_{2}$, lactic ac.

acetic ac., acetylmethyl carbinol, acetone, butyl alc., butyric ac., $\mathrm{CO}_{2}$, ethyl alc., formic ac., $\mathrm{H}_{2}$, lactic ac.

acetic ac., acetylmethyl carbinol, 2, 3-butylene glycol, ethyl alc., formic ac., lactic ac., succinic ac.

acetic ac., acetylmethyl carbinol, 2, 3-butylene glycol, $\mathrm{CO}_{2}$, ethyl alc., formic ac., $\mathrm{H}_{2}$, lactic ac., succinic ac.

acetic ac., acetylmethyl carbinol, 2, 3-butylene glycol, $\mathrm{CO}_{2}$, ethyl alc., formic ac., $\mathrm{H}_{2}$, lactic ac, succinic ac.

acetic ac., acetylmethyl carbinol, $\mathrm{CO}_{2}$, ethyl alc., $\mathrm{H}_{2}$, lactic ac., succinic ac.

Cl. pasteurianum

Donker, 1926

B. acetoethylicum

Arzberger, Peterson and Fred, I 920

B. polymyxa

Donker, I926

B. acetoethylicum

Donker, 1926

B. granulobacter pectinovorum (Weizmann)

Donker, I926

$B$. lactis aerogenes

Harden and Walpole, I 906

$B$. aerogenes

Scheffer, 1928

Serratia marcescens

Pederson and Breed, 1928

B. tartricus

Grimbert, I90 I 


\begin{tabular}{|c|c|}
\hline $\begin{array}{l}\text { Substrates } \\
\text { Products }\end{array}$ & $\begin{array}{l}\text { Microorganisms } \\
\text { Authors }\end{array}$ \\
\hline \multicolumn{2}{|l|}{ Dextrose (cont.) } \\
\hline $\begin{array}{l}\text { acetic ac., 2, 3-butylene glycol, } \\
\text { butyric ac., } \mathrm{CO}_{2} \text {, formic ac., } \\
\mathrm{H}_{2} \text {, formic ac. }\end{array}$ & $\begin{array}{l}\text { Granulobacter saccharo-butyricum } \\
\text { Donker, I926 }\end{array}$ \\
\hline acetic ac., butyric ac. & $\begin{array}{l}\text { Cl. pasteurianum } \\
\text { Winogradsky, } 1902\end{array}$ \\
\hline $\begin{array}{l}\text { acetic ac., butyric ac., } \mathrm{CO}_{2}, \\
\text { ethyl alc., formic ac., } \mathrm{H}_{2}, \\
\text { lactic ac., succinic ac. }\end{array}$ & $\begin{array}{c}\text { Sarcina maxima } \\
\text { Smit, I } 928\end{array}$ \\
\hline $\begin{array}{l}\text { acetic ac., butyric ac., } \mathrm{CO}_{2}, \\
\text { ethyl alc., formic ac., } \mathrm{H}_{2}, \\
\text { lactic ac., succinic ac. }\end{array}$ & $\begin{array}{l}\text { Sarcina ventriculi } \\
\text { Smit, I928 }\end{array}$ \\
\hline acetic ac., butyric ac., lactic ac. & $\begin{array}{c}\text { B. butylicus } \\
\text { Buchner and Meisenheimer, } \\
\text { I908 }\end{array}$ \\
\hline $\begin{array}{l}\text { acetic ac., } \mathrm{CO}_{2} \text {, ethyl alc., } \\
\text { gluconic ac. }\end{array}$ & $\begin{array}{l}\text { Acetobacter } S p . ? \\
\quad \text { Söhngen, I914, I915 }\end{array}$ \\
\hline acetic ac., $\mathrm{CO}_{2}$, ethyl alc., $\mathrm{H}_{2}$ & $\begin{array}{l}\text { B. ethaceticus } \\
\text { Frankland and Lumsden, } 189^{2}\end{array}$ \\
\hline $\begin{array}{l}\text { acetic ac., } \mathrm{CO}_{2} \text {, ethyl alc., } \\
\text { formic ac., } \mathrm{H}_{2} \text {, lactic ac., } \\
\text { succinic ac. }\end{array}$ & $\begin{array}{l}\text { B. coli communis } \\
\text { Grey antd Young, I92 I }\end{array}$ \\
\hline $\begin{array}{l}\text { acetic ac,. } \mathrm{CO}_{2} \text {, ethyl alc., } \\
\text { formic ac., } \mathrm{H}_{2} \text {, lactic ac., } \\
\text { succinic ac. }\end{array}$ & $\begin{array}{l}\text { B. Freundii } \\
\text { Scheffer, } 1928\end{array}$ \\
\hline $\begin{array}{l}\text { acetic ac., } \mathrm{CO}_{2} \text {, ethyl alc., } \\
\text { formic ac., } \mathrm{H}_{2} \text {, lactic ac., } \\
\text { succinic ac. }\end{array}$ & $\begin{array}{l}\text { B. coli communis } \\
\text { Grey, } 1920\end{array}$ \\
\hline $\begin{array}{l}\text { acetic ac., } \mathrm{CO}_{2} \text {, ethyl alc. } \\
\text { formic ac., lactic ac., suc- } \\
\text { cinic ac. }\end{array}$ & $\begin{array}{l}\text { B. cloacae, B. coli, } \\
\text { B. lactis aerogenes } \\
\text { Thompson, I } 9 \text { I } 2\end{array}$ \\
\hline $\begin{array}{l}\text { acetic ac., } \mathrm{CO}_{2} \text {, ethyl alc., } \mathrm{H}_{2}, \\
\text { lactic ac., succinic ac. }\end{array}$ & $\begin{array}{l}\text { B. coli communis } \\
\text { Harden, I } 90 \mathrm{I}\end{array}$ \\
\hline
\end{tabular}


Substrates

Products

Dextrose (cont.)

acetic ac., $\mathrm{CO}_{2}$, ethyl alc., $\mathrm{H}_{2}$, lactic ac., succinic ac.

acetic ac., $\mathrm{CO}_{2}$, ethyl alc., formic ac., $\mathrm{H}_{2}$, lactic ac., succinic ac.

acetic ac., $\mathrm{CO}_{2}$, ethyl alc., formic ac., lactic ac., succinic ac.

acetic ac., $\mathrm{CO}_{2}$, ethyl alc., $\mathrm{H}_{2}$, lactic ac.

acetic ac., $\mathrm{CO}_{2}$, ethyl alc., formic ac., $\mathrm{H}_{2}$

acetic ac., $\mathrm{CO}_{2}$, propionic ac., succinic ac.

acetic ac., ethyl alc.

acetic ac., ethyl alc., formic ac.

acetic ac., ethyl alc., formic ac.

acetic ac., ethyl alc., formic ac., lactic ac., succinic ac.

acetic ac., ethyl alc., succinic

ac.
Microorganisms

AUTHORS

B. coli communis

Grey, I9I 8

$B$. aerogenes, $B$. coli

Virtanen and Simola, I927

B. coli communis

Kay, I 926

B. coli communis

Harden, I 899

Friedlander's pneumococcus

Frankland, Stanley and Frew, I $89 \mathrm{I}$

Propionibacterium Freudenreichii

Propionibacterium Fensenii

Propionibacterium pentosaceum

Propionibacterium Peterssonii

Propionibacterium rubrum

Propionibacterium Shermanii

Propionibacterium technicum

Propionibacterium Thönii

Van Niel, I 928

Lactobacillus pentoaceticus

Peterson and Fred, I920

Bact. pyocyaneus

Aubel, I92 I

B. typhosus

Harden, Igo I

B. typhosum

Scheffer, I 928

Friedländer's pneumobacillus

Grimbert, I 896 


\begin{tabular}{l} 
Substrates \\
Products \\
\hline
\end{tabular}

Dextrose (cont.)

acetic ac., ethyl alc., formic ac., lactic ac., succinic ac. acetic ac., ethyl alc., l-lactic ac. acetic ac., lactic ac., succinic ac. acetic ac, oxalic ac.

acetic ac., propionic ac., succinic ac.

acetone, ethyl alc., formic ac.

acetone, ethyl alc., pyruvic ac. acetylmethyl carbinol

acetic ac., acetylmethyl carbinol, $\mathrm{CO}_{2}$, ethyl alc., formic ac., lactic ac., succinic ac. acetylmethyl carbinol, 2, 3butylene glycol

acetylmethyl carbinol, 2, 3butylene glycol

acetylmethyl carbinol, 2, 3butylene glycol

acetylmethyl carbinol, 2, 3butylene glycol

acetylmethyl carbinol, 2, 3butylene glycol
Microorganisms

Authors

B. dysenteriae, Shiga-Kruse

Scheffer, 1928

B. coli

Grimbert, I 896

$B$. coli communis

Young, Iy 24

Asp. niger

Heinze, 1903

B. acidi propionici var. fuscum

Virtanen, I923

$B$. acetoethylicum

Northrop, Ashe and Morgan, I919

B. acetoethylicum

Speakman, I925

B. mesentericus vulgatus

B. subtilis

Tyrothrix tenuis

Harden and Norris, I9I 2

$B$. lactis aerogenes

$\mathrm{Kay},{ }_{926}$

$B$. lactis aerogenes

Walpole, I9 I I

B. cloacae

Thompson, I 9 I 2

$B$. lactis aerogenes

Harden and Norris, I9I 2

$B$. lactis aerogenes

Neuberg, Nord, and Wolff, I 920

B. proteus

Lemoigne, I 923 


\begin{tabular}{|c|c|}
\hline $\begin{array}{l}\text { Substrates } \\
\text { Products }\end{array}$ & $\begin{array}{l}\text { Microorganisms } \\
\text { AUthors }\end{array}$ \\
\hline \multicolumn{2}{|l|}{ Dextrose (cont.) } \\
\hline $\begin{array}{l}\text { acetylmethyl carbinol, 2, 3- } \\
\text { butylene glycol }\end{array}$ & $\begin{array}{l}\text { yeast } \\
\text { Kluyver, Donker and Hooft, } \\
\text { I } 9^{25}\end{array}$ \\
\hline $\begin{array}{l}\text { acetylmethyl carbinol, 2, 3- } \\
\text { butylene glycol, } \mathrm{CO}_{2} \text {, ethyl } \\
\text { alc., formic ac., } \mathrm{H}_{2} \text {, lactic } \\
\text { ac. }\end{array}$ & $\begin{array}{l}\text { B. cloacae } \\
\text { Scheffer, I928 }\end{array}$ \\
\hline butyric ac. & $\begin{array}{c}\text { Bact. thermobutyricus } \\
\text { Coolhaas, I } 928\end{array}$ \\
\hline $\begin{array}{l}\text { butyric ac., butyl alc., caproic } \\
\text { ac., caprylic ac. }\end{array}$ & $\begin{array}{l}\text { B. butylicus } \\
\text { Neuberg and Arinstein, I92 I }\end{array}$ \\
\hline butyric ac., $\mathrm{CO}_{2}, \mathrm{H}_{2}$, mannitol & $\begin{array}{l}\text { B. suotilis } \\
\text { Vanderwelde, I } 88_{4}\end{array}$ \\
\hline citric ac. & $\begin{array}{l}\text { Asp. fumaricus } \\
\text { Schreyer, } 1928\end{array}$ \\
\hline citric ac. & $\begin{array}{l}\text { Citromyces citricus } \\
\text { Citromyces lacticus } \\
\text { Citromyces tartricus } \\
\text { Citromyces oxalicus } \\
\quad \text { Mazé and Perrier, I9O4 }\end{array}$ \\
\hline citric ac. & $\begin{array}{l}\text { Citromyces, sp.? } \\
\text { Martin, I9I6 }\end{array}$ \\
\hline citric ac. & $\begin{array}{l}\text { Asp. niger } \\
\text { Bernhauer, I } 928\end{array}$ \\
\hline citric ac. & $\begin{array}{l}\text { Citromyces citricus } \\
\text { Buchner and Wüstenfeld, } \\
\text { Igog }\end{array}$ \\
\hline citric ac. & $\begin{array}{l}\text { Citromyces Pfefferianus } \\
\text { Buchner and Wüstenfeld, I909 }\end{array}$ \\
\hline citric ac. & $\begin{array}{l}\text { Mucor piriformis } \\
\text { Wehmer, I } 897\end{array}$ \\
\hline citric ac. & $\begin{array}{l}\text { Pen. Iuteum } \\
\text { Wehmer, } 1897\end{array}$ \\
\hline
\end{tabular}




\begin{tabular}{|c|c|}
\hline $\begin{array}{l}\text { Substrates } \\
\text { Products }\end{array}$ & $\begin{array}{l}\text { Microorganisms } \\
\text { Authors }\end{array}$ \\
\hline \multicolumn{2}{|l|}{ Dextrose (cont.) } \\
\hline citric ac., gluconic ac. & $\begin{array}{l}\text { Asp. niger, Asp. cinnamoneus, } \\
\text { Asp. fuscus } \\
\text { Falck and Kapur, } 1924\end{array}$ \\
\hline citric ac., oxalic ac. & $\begin{array}{l}\text { Asp. niger } \\
\quad \text { Wehmer, } 192.4\end{array}$ \\
\hline citric ac., oxalic ac. & $\begin{array}{l}\text { Asp. niger } \\
\quad \text { Amelung, } 1927\end{array}$ \\
\hline citric ac., oxalic ac. & $\begin{array}{l}\text { Asp. niger } \\
\text { Elfving, I9I9 }\end{array}$ \\
\hline citric ac., oxalic ac. & $\begin{array}{l}\text { Citromyces glaber } \\
\text { Butkewitsch, I922 }\end{array}$ \\
\hline $\mathrm{CO}_{2}$, ethyl alc. & $\begin{array}{l}\text { Fusarium lini } \\
\text { Anderson and Willaman, } 1922\end{array}$ \\
\hline ethyl alc. & $\begin{array}{l}\text { Mucor mucedo } \\
\text { Mucor racemosus } \\
\text { Mucor stolonifer } \\
\text { Kostytschew and Eliasberg, } \\
\quad 1920\end{array}$ \\
\hline ethyl alc. & $\begin{array}{l}\text { yeast } \\
\text { Rimini, } 1926\end{array}$ \\
\hline ethyl alc. & $\begin{array}{l}\text { Rhizopus, sp.? } \\
\text { Takahashi and Sakaguchi, } \\
\text { I927 }\end{array}$ \\
\hline ethyl alc., $\mathrm{CO}_{2}$ & $\begin{array}{l}\text { Asp. niger } \\
\text { Kostytschew, } 1907\end{array}$ \\
\hline formic ac. & $\begin{array}{l}\text { B. prodigiosus } \\
\text { Franzen and Egger, I91 } 2\end{array}$ \\
\hline formic ac., $\mathrm{CO}_{2}, \mathrm{H}_{2}$ & $\begin{array}{l}\text { B. coli communis } \\
\text { Franzen and Kahlenberg, } \\
\text { I9I6 }\end{array}$ \\
\hline fumaric ac. & $\begin{array}{l}\text { Rhizopus nigricans } \\
\text { Ehrlich, I9I2 }\end{array}$ \\
\hline gluconic ac. & $\begin{array}{l}\text { Asp. niger } \\
\text { Bernhauer, } 1928\end{array}$ \\
\hline
\end{tabular}




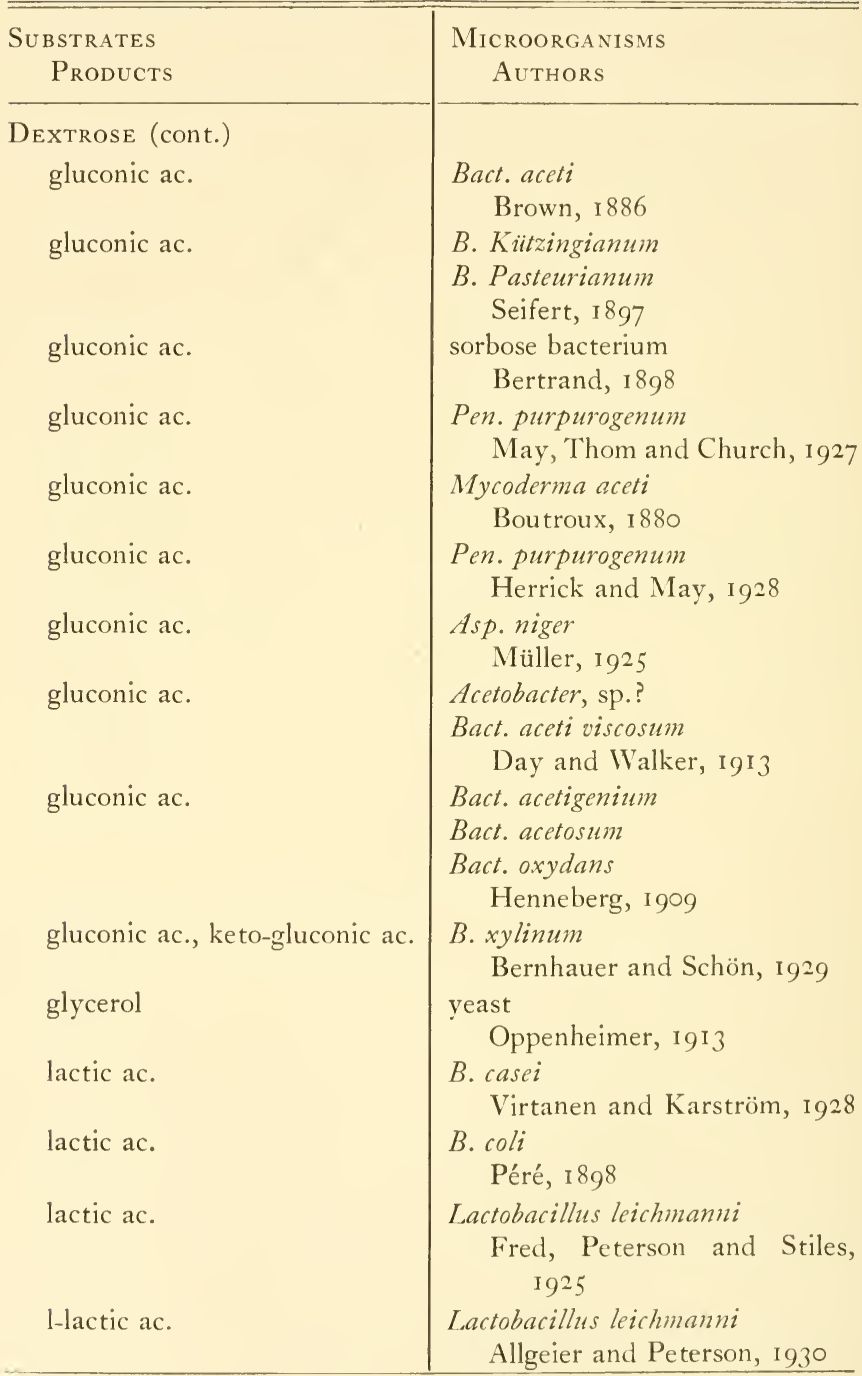




\begin{tabular}{|c|c|}
\hline $\begin{array}{l}\text { Substrates } \\
\text { Products }\end{array}$ & $\begin{array}{c}\text { Microorganisms } \\
\text { Authors }\end{array}$ \\
\hline \multicolumn{2}{|l|}{ Dextrose (cont.) } \\
\hline lactic ac. & $\begin{array}{l}\text { Lactobacillus, sp.? } \\
\quad \text { Neuberg and Gorr, } 1926\end{array}$ \\
\hline d-lactic ac. & $\begin{array}{l}\text { Clostridium thermocellum } \\
\text { Peterson, Fred and Martin, } \\
\text { I926 }\end{array}$ \\
\hline d-lactic ac. & $\begin{array}{l}\text { Strept. lactis } \\
\quad \text { Allgeier and Peterson, I } 930\end{array}$ \\
\hline lactic ac. & $\begin{array}{l}\text { Lactobacillus pentoaceticus } \\
\text { Peterson and Fred, I } 920\end{array}$ \\
\hline lactic ac. & $\begin{array}{l}\text { B. propionicus } \\
\text { Neuberg and Gorr, I926 }\end{array}$ \\
\hline lactic ac. & $\begin{array}{l}\text { yeast } \\
\text { Aubel, } 1929\end{array}$ \\
\hline lactic ac., $\mathrm{CO}_{2}$ & $\begin{array}{l}\text { B.coli communis } \\
\quad \text { Goto, } 1925\end{array}$ \\
\hline lactic ac., ethyl alc. & $\begin{array}{l}\text { B. of malignant oedema } \\
\text { King and Frankel, I } 890\end{array}$ \\
\hline 1-lactic ac. & $\begin{array}{l}\text { Rhizopus chinensis } \\
\text { Saito, I9I I }\end{array}$ \\
\hline lactic ac. & $\begin{array}{l}\text { yeast } \\
\quad \text { Oppenheimer, I9I3 }\end{array}$ \\
\hline oxalic ac. & $\begin{array}{l}\text { Asp. niger } \\
\quad \text { Wehmer, 1891, I897 }\end{array}$ \\
\hline oxalic ac. & $\begin{array}{l}\text { B. aceti, } B . \text { Kïtzingianum } \\
\text { B. acetigenum, B. acetosum } \\
\text { B. ascendens, B. industrium } \\
\text { B. oxydans, B. Pasteurianum } \\
\text { B. xylinium, Termobacterium acet } i \\
\quad \text { Banning, I902 }\end{array}$ \\
\hline oxygluconic ac. & $\begin{array}{l}\text { Micrococcus oblongus } \\
\text { Boutroux, I } 886\end{array}$ \\
\hline propionic. ac. & $\begin{array}{c}\text { Diplococcus pneumoniae } \\
\text { Brieger, } 1883\end{array}$ \\
\hline pyruvic ac. & $\begin{array}{l}\text { yeast } \\
\text { Traetta-Mosca, } 1927\end{array}$ \\
\hline
\end{tabular}




Substrates
Products

Dextrose (cont.)

pyruvic ac.

pyruvic ac.

pyruvic ac.

$\alpha, \alpha$, DICH LOROACETONE

$\alpha, \alpha$-di-chloropropylalcohol

Dihydroxyacetone

dextrose

lactic ac.

DiHydRoXYMALEIC AC.

$\mathrm{CO}_{2}$, glycollic aldehyde

Du LCITOL

acetic ac., $\mathrm{CO}_{2}$, ethyl alc., $\mathrm{H}_{2}$, succinic ac.

ENANTHOL

n-heptyl alc.

ERYTHRITOL

erythrulose

erythrose

oxalic ac.
Microorganisms

Authors

Mycoderma, sp.?

Fernbach and Schoen, I9I4 B. coli

Aubel, I 926

yeast

Fernbach and Schoen, I920

yeast

Sen, $19^{2} 4$

Sacc. ludwigii

Neuberg and Kobel, 1928 yeast

Oppenheimer, I9I3

yeast

Neuberg and Schwenk, I 9 I 5

B. ethoacetosuccinicus

Frankland and Frew, 1892

yeast

Ohta, I9I 4

sorbose bacterium

Bertrand, I 898, I 900

Acetobacter suboxydans

Kluyver and deLeeuw, 1924

B. aceti, B. acctosum

$B$. industrium, B. oxydans

Banning, 1902 


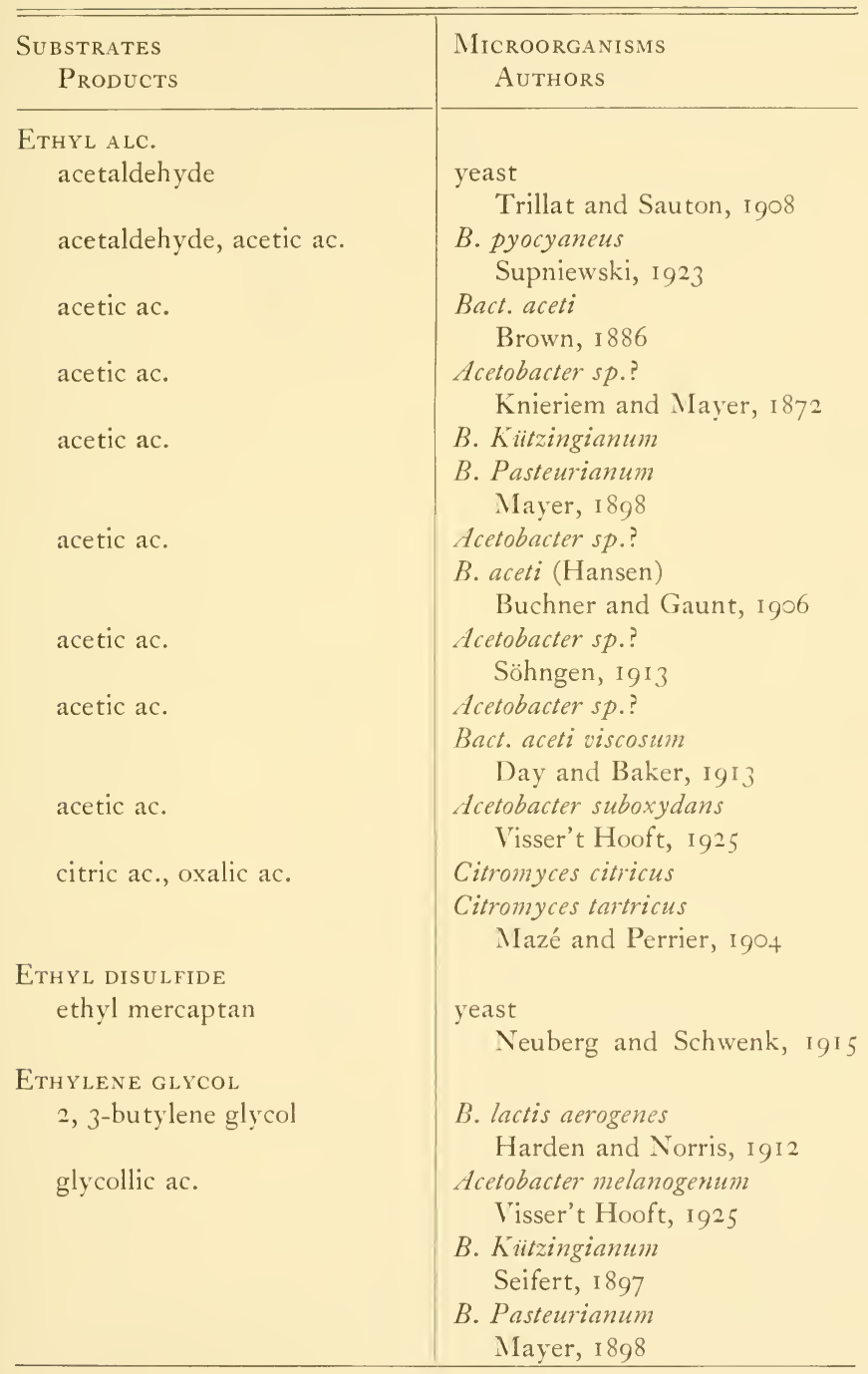


Substrates

Products

Ethylene glycol (cont.) oxalic ac.

FormaLDEHYDE

methyl alc.

Formic ac.

$\mathrm{CO}_{2}, \mathrm{H}_{2}$

Fumaric ac.

acetic ac., pyruvic ac.

l-malic ac.

malic ac.

oxalic ac.

pyruvic ac.

succinic ac.

Furfural

furfuryl alc.

furyltrimethylene glycol

Galactose

acetaldehyde
Microorganisms

Authors

B. aceti, B. acetosum

$B$. ascendens, $B$. industrium

B. Kützingianum, B. oxydans

B. Pasteurianum

Termobacterium aceti

Banning, 1902

yeast

Neuberg and Welde, $19^{14}$

B. coli communis

Franzen and Kahlenberg, I 9 I 6

B. pyocyaneus

Quastel, 1924

Asp. niger

Challenger and Klein, 1929

B. coli

Quastel and Whetham, 1924 Asp. niger

Raistrick and Clark, I9I9

$B$. pyocyaneus

Quastel, I924

B. coli communis

Quastel, Stephenson and Whetham, I925

yeast

Lintner and Liebig, I9I yeast

Lintner and Liebig, I913

Bact. coli, B. lactis aerogenes

Nagai, 1923 
Substrates

Products

Galactose (cont.)

acetaldehyde, $\mathrm{CO}_{2}$, glycerol

acetic ac., acetone, butyl alc.,

butyric ac., lactic ac.

acetic ac., $\mathrm{CO}_{2}$, ethyl alc., $\mathrm{H}_{2}$,

lactic ac., succinic ac.

acetic ac., ethyl alc., lactic ac.

acetone, ethyl alc, formic ac.

acetylmethyl carbinol, 2, 3butylene glycol citric ac, oxalic ac.

fumaric ac., citric ac.

galactonic ac.

lactic ac.

oxalic ac.

$\alpha$-Glucoheptite

$\alpha$-glucoheptulose

Gluconic ac.

acetaldehyde

acetic ac., ethyl alc., formic ac. lactic ac., succinic ac.

acetic ac., acetylmethyl carbinol, $\mathrm{CO}_{2}$, ethyl alc., formic ac., lactic ac., succinic ac.
Microorganisms

Authors

yeast

Tomita, I92 I

B. granulobacter pectinovorum

Speakman, I923

B. coli communis

Harden, I 90 I

Lactobacillus pentoaceticus

Peterson and Fred, 1920

B. acetoethylicum

Northrop, Ashe and Morgan, I9I9

B. lactis aerogenes

Harden and Norris, I9I 2

Asp. niger

Amelung, 1927

Asp. fumaricus

Schreyer, 1928

sorbose bacterium

Bertrand, 1898

B. coli

Péré, I 898

B. Kiitzingianum

Termobacterium aceti

Banning, 1902

sorbose bacterium

Bertrand and Nitzberg, I 928

B. lactis aerogenes

Nagai, 1923

B. coli communis

Kay, 1926

$B$. lactis aerogenes

Kay, I926 


Substrates
Products

Gluconic AC. (cont.)

citric ac.

citric ac., saccharic ac.

dihydroxyacetone, ethyl alc.

oxalic ac.

oxygluconic ac.

\section{Glyceraldehyde}

lactic ac.

Glyceric AC.

acetaldehyde

acetaldehyde

acetaldehyde, acetic ac., $\mathrm{CO}_{2}$, ethyl alc.

citric ac.

Glycerol

acetaldehyde

acetic ac., butyl alc., butyric ac.

acetic ac., 2, 3-butylene glycol,

$\mathrm{CO}_{2}$, ethyl alc., formic ac.

$\mathrm{H}_{2}$, lactic ac., succinic ac.

acetic ac., 2, 3-butylene glycol,

$\mathrm{CO}_{2}$, ethyl alc., formic ac., $\mathrm{H}_{2}$, lactic ac., succinic ac.
Microorganisms

Authors

Asp. fumaricus

Schreyer, I925, I 928

Asp. niger

Walker, Subramaniam and Challenger, I 927

Acetobacter sp.?

Söhngen, I9I4, I9I 5

Asp. niger

Butkewitsch, I 923

Acetobacter suboxydans

Kluyver and deLeeuw, I92 I

yeast

Oppenheimer, I9I3

B. lactis aerogenes

Nagai, I923

yeast

Lebedev and Polonski, I9I6 yeast

Lebedev, I91 8

Asp. fumaricus

Schreyer, 1928

$B$. lactis aerogenes

Kumagawa, 1922

Amylobacter butylicus

Duclaux, I 895

$B$. lactis aerogenes

Harden and Norris, I9I 2

Bact. aerogenes

Braak, 1928 


\section{Substrates}

Products
Microorganisms

Authors

Glycerol (cont.)

acetic ac., $\mathrm{CO}_{2}$, ethyl alc., formic ac., $\mathrm{H}_{2}$ lactic ac., succinic ac., trimethylene glycol acetic ac., $\mathrm{CO}_{2}$, ethyl alc., formic ac., $\mathrm{H}_{2}$, lactic ac., succinic ac.

acetic ac., $\mathrm{CO}_{2}$, propionic ac., succinic ac. acetic ac., $\mathrm{CO}_{2}$, propionic ac.

acetic ac., ethyl alc., formic ac., succinic ac. acetic ac., $\mathrm{CO}_{2}$, ethyl alc., formic ac., lactic ac., succinic ac.

acetone, ethyl alc., formic ac.

acetylmethyl carbinol

acrolein

acrylaldehyde

n-amyl alc., n-butyl alc., npropyl alc.

butyl alc.

butyl alc., butyric ac., lactic ac.

butyl alc., butyric ac., caproic ac., $\mathrm{CO}_{2}$, ethyl alc., $\mathrm{H}_{2}$

Bact. Freundii

Braak, 1928

Bact. coli

Braak, 1928

Propionibacterium pentosaceum

Van Niel, 1928

Propionibacterium Thonii

Propionibacterium technicum

Propionibacterium Shermanii

Propionibacterium Peterssonii

Propionibacterium Fensenii

Van Niel, 1928

B. ethaceticus

Frankland and Fox, I 889

B. coli

Grey, 1923

B. acetoethylicum

Northrop, Ashe and Morgan, IgI9

Tyrothrix tenuis

Harden and Norris, 1912

B. Welchii

Humphreys, 1924

B. amaracrylus

Voisenet, 1918

B. butylicus

B. butylicus

Morin, 1887

Emmerling, I 897

B. butylicus

Fitz, 1882

Mucor racemosus

Fitz, 1876 


\section{Substrates}

PRODUCTS

Glycerol (cont.)

n-butyl alc., formic ac., lactic ac.

2, 3-butylene glycol

butyl alc., butyric ac., caproic ac., caprylic ac.

butyric ac., lactic ac., succinic ac.

citric ac.

citric ac.

citric ac., oxalic ac.

$\mathrm{CO}_{2}$, glyceric ac., lactic ac.

dihydroxyacetone

dihydroxyacetone

dihydroxyacetone

dihydroxyacetone

dihydroxyacetone

dihydroxyacetone

dihydroxyacetone

dihydroxyacetone
Microorganisms

Authors

B. butylicus

Buchner and Meisenheimer, Igo8

$B$. lactis aerogenes

Harden and Norris, I9I 2

$B$. butylicus

Neuberg and Arinstein, I92 I

B. subtilis

$\mathrm{V}$ andewelde, I $88_{4}$

Citromyces, sp.?

Wehmer, I9I3

Citromyces citricus

Mazé and Perrier, I904

Asp. niger

Citromyces glaber

Butkewitsch, I923

B. pyocyaneus

Supniewski, I923

Acetobacter suboxydans

Kluyver and deLeeuw, I924 sorbose bacterium

Bertrand, I904

$B$. dioxyacetonicum

Virtanen and Barlund, I926 sorbose bacterium

Bertrand, 1898

B. xylinum

Virtanen and Barlund, I926

B. xylinum

Bernhauer and Schön, I928

Acetobacter suboxydans

Bact. xylinum

Brit. patent 269,950

Bact. xylinum

Bertrand and Sazerac, I go I 


\begin{tabular}{|c|c|}
\hline $\begin{array}{l}\text { Substrates } \\
\text { Products }\end{array}$ & $\begin{array}{l}\text { Microorganisms } \\
\text { Authors }\end{array}$ \\
\hline \multicolumn{2}{|l|}{$\begin{array}{l}\text { GLYCERol (cont.) } \\
\text { ethyl alc. }\end{array}$} \\
\hline ethyl alc. & $\begin{array}{l}\text { yeast } \\
\text { Neuberg and Kerb, I9I3 }\end{array}$ \\
\hline ethyl alc. & $\begin{array}{l}\text { Asp. niger } \\
\text { Kostytschew and Afanass- } \\
\text { jewa, I } 922\end{array}$ \\
\hline ethyl alc., $\mathrm{CO}_{2}$ & $\begin{array}{l}\text { yeast } \\
\text { Kostytschew and Faermann, } \\
\text { I928 }\end{array}$ \\
\hline gluconic ac. & $\begin{array}{l}\text { Asp. niger } \\
\text { Bernhauer, I } 928\end{array}$ \\
\hline oxalic ac. & $\begin{array}{l}\text { B. aceti, B. ascendens } \\
\text { B. oxydans, B. Pasteurianum } \\
\text { B. xylinum } \\
\text { Termobacterium aceti } \\
\quad \text { Banning, } 1902\end{array}$ \\
\hline oxalic ac. & $\begin{array}{l}\text { Asp. niger } \\
\text { Elfving, I9I9 }\end{array}$ \\
\hline oxymaltol & $\begin{array}{l}\text { Asp. glaucus } \\
\quad \text { Traetta-Mosca and Preti, I } 92 \text { I }\end{array}$ \\
\hline pyruvic ac. & $\begin{array}{l}\text { B. subtilis } \\
\text { Aubel, I921 }\end{array}$ \\
\hline succinic ac. & $\begin{array}{l}\text { Rhizopus, sp.? } \\
\text { Takahashi and Sakaguchi, } \\
\text { I } 9^{27}\end{array}$ \\
\hline sucrose & $\begin{array}{l}\text { yeast } \\
\text { Connstein and Ludecke, I } 9 \text { I } 9\end{array}$ \\
\hline $\begin{array}{l}\text { GLycerose } \\
\text { citric ac., oxalic ac. }\end{array}$ & $\begin{array}{l}\text { Asp. niger } \\
\text { Amelung, } 1927\end{array}$ \\
\hline $\begin{array}{l}\text { GLYCOLALDEHYDE } \\
\text { ethylene glycol }\end{array}$ & $\begin{array}{l}\text { yeast } \\
\quad \text { Neuberg and Schwenk, I9I } 5\end{array}$ \\
\hline $\begin{array}{l}\text { GLYCOLLIC AC. } \\
\text { oxalic ac. }\end{array}$ & $\begin{array}{l}\text { B. aceti, } B . \text { acetigenum } \\
\text { B. acetosum, } B . \text { ascendens } \\
B . \text { Kiitzingianum }\end{array}$ \\
\hline
\end{tabular}




\begin{tabular}{|c|c|}
\hline $\begin{array}{l}\text { Substrates } \\
\text { Products }\end{array}$ & $\begin{array}{l}\text { Microorganisms } \\
\text { Authors }\end{array}$ \\
\hline GLycollic AC. (cont.) & $\begin{array}{l}\text { B. Pasteurianum } \\
\text { Termobacterium aceti } \\
\quad \text { Banning, I } 902\end{array}$ \\
\hline $\begin{array}{l}\text { GLYcuRonic Ac. } \\
\text { acetic ac., } \mathrm{CO}_{2} \text {, ethyl alc., } \\
\text { formic ac., lactic ac., suc- } \\
\text { cinic ac. }\end{array}$ & $\begin{array}{l}\text { B. coli communis } \\
\text { Kay, I } 926\end{array}$ \\
\hline $\begin{array}{l}\text { GLYOXYLIC AC. } \\
\text { acetaldehyde, } \mathrm{CO}_{2}\end{array}$ & $\begin{array}{l}\text { yeast } \\
\quad \text { Lebedev, I9I } 8\end{array}$ \\
\hline $\begin{array}{l}\text { Hydroxyfumaric Ac. } \\
\text { acetaldehyde, } \mathrm{CO}_{2}\end{array}$ & $\begin{array}{l}\text { yeast } \\
\text { Mayer, I9I3 }\end{array}$ \\
\hline $\begin{array}{l}\text { Hydroxypyruvic ac. } \\
\text { glycolaldehyde, } \mathrm{CO}_{2}\end{array}$ & $\begin{array}{l}\text { yeast } \\
\text { Neuberg and Kerb, I9I3 }\end{array}$ \\
\hline $\begin{array}{l}\text { InOSitol } \\
\text { acetaldehyde, } \mathrm{CO}_{2} \text {, lactic ac., } \\
\text { succinic ac. }\end{array}$ & $\begin{array}{l}\text { B. lactis aerogenes } \\
\text { Kumagawa, } 1922\end{array}$ \\
\hline $\begin{array}{l}\text { i-Inositol } \\
\text { acetic ac., } \mathrm{CO}_{2} \text {, ethyl alc., } \\
\text { succinic ac. }\end{array}$ & $\begin{array}{l}\text { B. lactis } \\
\text { Hewitt and Steabbin, I } 92 \text { I }\end{array}$ \\
\hline $\begin{array}{l}\text { INULIN } \\
\quad \text { oxalic ac. }\end{array}$ & $\begin{array}{l}\text { Asp. niger } \\
\text { Elfving, I9I9 }\end{array}$ \\
\hline $\begin{array}{l}\text { IsoBUTYL ALC. } \\
\text { isobutyric ac. }\end{array}$ & $\begin{array}{l}\text { Acetobacter melanogenum } \\
\text { Visser't Hooft, } 1925\end{array}$ \\
\hline $\begin{array}{l}\text { ISOBUTYL ALDEHYDE } \\
\text { isobutyl alc. }\end{array}$ & $\begin{array}{l}\text { yeast } \\
\text { Ohta, I9I4 }\end{array}$ \\
\hline $\begin{array}{l}\text { IsOBUTYRIC Ac. } \\
\text { oxalic ac. }\end{array}$ & $\begin{array}{l}\text { B. aceti, B. Kiitzingianum } \\
\text { B. Pasteurianum } \\
\text { Banning, } 1902\end{array}$ \\
\hline
\end{tabular}




\begin{tabular}{|c|c|}
\hline $\begin{array}{l}\text { Substrates } \\
\text { Products }\end{array}$ & $\begin{array}{l}\text { Microorganisms } \\
\text { Authors }\end{array}$ \\
\hline $\begin{array}{l}\text { ISODULCITE } \\
\text { See rhamnose }\end{array}$ & \\
\hline $\begin{array}{l}\text { IsoLICHENIN } \\
\text { oxalic ac. }\end{array}$ & $\begin{array}{l}\text { B. aceti, } B . \text { industrium } \\
\text { B. Kützingianum } \\
\text { Banning, } 1902\end{array}$ \\
\hline $\begin{array}{l}\text { ISOPROPYL ALC. } \\
\text { acetone }\end{array}$ & $\begin{array}{l}\text { Acetobacter melanogenum } \\
\text { Acetobacter xylinum } \\
\quad \text { visser't Hooft, } 1925\end{array}$ \\
\hline $\begin{array}{l}\alpha \text {-Kетов Uty Ric Ac. } \\
\text { ethyl alc., n-propyl alc. } \\
\text { propionaldehyde } \\
\text { propionaldehyde, propyl alc. }\end{array}$ & $\begin{array}{l}\text { yeast } \\
\text { Neuberg and Kerb, I9I4 } \\
\text { yeast } \\
\text { Neuberg and Kerb, I9I } 2 \\
\text { yeast } \\
\text { Neuberg and Kerb, I9I3 }\end{array}$ \\
\hline $\begin{array}{l}\alpha \text {-KETO-ISO-VALERIC AC. } \\
\text { isobutyl aldehyde }\end{array}$ & $\begin{array}{l}\text { yeast } \\
\text { Sen, } 19^{2} 3\end{array}$ \\
\hline $\begin{array}{l}\alpha \text {-KETO-n-CAPROIC Ac. } \\
\text { n-amyl alc., n-valeraldehyde }\end{array}$ & $\begin{array}{l}\text { yeast } \\
\text { Sen, } 19^{2} 3\end{array}$ \\
\hline $\begin{array}{l}\text { LActic Ac. } \\
\text { acetaldehyde }\end{array}$ & $\begin{array}{l}\text { B. lactis aerogenes } \\
\text { Nagai, } 19^{2} 3\end{array}$ \\
\hline $\begin{array}{l}\text { acetaldehyde, acetic ac., py- } \\
\text { ruvic ac. } \\
\text { acetic ac. }\end{array}$ & $\begin{array}{l}\text { B. pyocyaneus } \\
\text { Supniewski, } 1923 \\
\text { Lactobacillus pentoaceticus } \\
\text { Peterson and Fred, } 1920\end{array}$ \\
\hline acetic ac., butyl alc., butyric ac. & $\begin{array}{l}\text { Acetobacter melanogenum } \\
\text { Acetobacter suboxydans } \\
\text { Acetobacter xylinum } \\
\text { visser't Hooft, I } 925 \\
\text { Amylobacter butylicus } \\
\text { Duclaux, I } 895\end{array}$ \\
\hline
\end{tabular}


Substrates

Products

LActic ac. (cont.)

acetic ac., $\mathrm{CO}_{2}$, propionic ac.

acetic ac., $\mathrm{CO}_{2}$, propionic ac.

acetic ac., $\mathrm{CO}_{2}$, propionic ac., succinic ac.

acetic ac., ethyl alc., formic ac.

acetic ac., ethyl alc., 1-lactic ac., succinic ac.

acetic ac., propionic ac.

acetone, ethyl alc., formic ac.

acetylmethyl carbinol

2, 3-butylene glycol

butyl alc., butyric ac., caproic ac., caprylic ac. citric ac.

ethyl alc.
Microorganisms

Authors

Bact. acidi propionici var. fuscum

Virtanen, 1923

$B$. acidi propionici

Bact. acidi propionici-b

Bact. acidi propionici-a

Von Freudenreich and OrlaJensen, 1906

Propionibacterium Freudenreichii

Propionibacterium Fensenii

Propionibacterium pentosaceum

Propionibacterium Peterssonii

Propionibacterium rubrum

Propionibacterium Shermanii

Propionibacterium technicum

Propionibacterium Thönii

Van Niel, I928

B. ethoacetosuccinicus

Mazé, I9I 3

B. coli

Grimbert, 1896

B. motilis

Orla-Jensen, I904

B. acetoethylicum

Northrop, Ashe and Morgan, I919

B. subtilis

Lemoigne, 1923

B. mesenterious-ruber

Lemoigne, I9I 3

B. butylicus

Neuberg and Arinstein, I92 I

Asp. fumaricus

Schreyer, 1928

Asp. niger

Kostytschew and Afanassjewa, 1922 


\begin{tabular}{|c|c|}
\hline $\begin{array}{l}\text { Substrates } \\
\text { Products }\end{array}$ & $\begin{array}{c}\text { Microorganisms } \\
\text { Authors }\end{array}$ \\
\hline $\begin{array}{l}\text { propionic ac., valeric ac. } \\
\text { pyruvic ac. } \\
\text { pyruvic ac. }\end{array}$ & $\begin{array}{l}\text { yeast } \\
\text { Kayser, I923 } \\
\text { B. Kiitzingianum } \\
\text { Termobacterium aceti } \\
\quad \text { Banning, I902 } \\
\text { B. subtilis } \\
\text { Fitz, I } 880 \\
\text { Amylomyces Rouxii } \\
\text { Mazé and Ruot, I916 } \\
\text { B. coli communis } \\
\text { B. pyocyaneus } \\
\text { Quastel, Stephenson and } \\
\text { Whetham, I925 }\end{array}$ \\
\hline $\begin{array}{l}\text { LActosE } \\
\text { acetaldehyde } \\
\text { acetaldehyde } \\
\text { acetic ac., acetylmethyl car- } \\
\text { binol, } \mathrm{CO}_{2} \text {, ethyl alc., } \mathrm{H}_{2} \text {, } \\
\text { lactic ac., succinic ac. } \\
\text { acetic ac., butyl alc., butyric } \\
\text { ac., } \mathrm{CO}_{2} \text {, ethyl alc., } \mathrm{H}_{2} \text {, } \\
\text { lactic ac. } \\
\text { acetic ac., propionic ac. } \\
\text { acetic ac., propionic ac., suc- } \\
\text { cinic ac. } \\
\text { acetic ac., succinic ac. } \\
\text { citric ac. }\end{array}$ & $\begin{array}{l}\text { Bact. coli., B. lactis aerogenes } \\
\text { Nagai, I } 923 \\
\text { yeast } \\
\text { Trillat, I908 } \\
\text { B. tartricus } \\
\text { Grimbert, I90 I } \\
\text { Amylobacter butylicus } \\
\text { Duclaux, I } 895 \\
\text { Bact. acidi propionici } d \\
\text { Sherman and Shaw, } 1923 \\
\text { Bact. acidi propionici } \\
\text { Virtanen, I } 923 \\
\text { B. lactis aerogenes } \\
\text { Emmerling, Igoo } \\
\text { Citromyces, sp.? } \\
\text { Wehmer, I9 } 3 \\
\text { Lactobacillus leichmani } \\
\text { Fred, Peterson and Stiles, } \\
\quad \text { I925 }\end{array}$ \\
\hline
\end{tabular}




\begin{tabular}{l} 
Substrates \\
Products \\
\hline Lactose (cont.) \\
lactic ac. \\
lactic ac., methylglyoxal, py- \\
ruvic ac. \\
oxalic ac.
\end{tabular}

Microorganisms

AUTHORS

B. coli

Péré, 1898

Bact. caucasicum

Kostytschew and SoldaAsp. niger tenkov, 1927

Elfving, I9I9

Bact. coli, B. lactis aerogenes

Nagai, 1923

acetic ac., butyric ac., $\mathrm{CO}_{2}$, Sarcina maxima ethyl alc., formic ac., $\mathrm{H}_{2}$, lactic ac., succinic ac.

acetic ac., $\mathrm{CO}_{2}$, ethyl alc., $\mathrm{H}_{2}$, lactic ac., succinic ac.

acetic ac., ethyl alc., formic ac., lactic ac.

acetic ac., lactic ac., mannitol

acetone, ethyl alc., formic ac.

acetylmethyl carbinol, 2, 3butylene glycol acetylmethyl carbinol, 2, 3butylene glycol acetylmethyl carbinol, 2, 3butylene glycol

acetylmethyl carbinol

2,3-butylene glycol

acetylmethyl carbinol citric ac., oxalic ac.
Smit, 1928

B. coli communis

Harden, I90 I

Bact. pyocyaneus

Aubel, I92I

Lactobacillus pentoaceticus

Peterson and Fred, 1920

B. acetoethylicum

Northrop, Ashe and Morgan, 1919

$B$. lactis aerogenes

Walpole, I9I I

B. cloacae

Harden and Norris, I $9^{12}$ yeast

Kluyver, Donker and Hooft, I 925

yeast

Kluyver and Donker, 1924 yeast

Neuberg and Reinfurth, 1923 Asp. niger

Amelung, 1927 


\begin{tabular}{|c|c|}
\hline $\begin{array}{l}\text { Substrates } \\
\text { Products }\end{array}$ & $\begin{array}{l}\text { Microorganisms } \\
\text { AUTHORS }\end{array}$ \\
\hline \multicolumn{2}{|l|}{ Levulose (cont.) } \\
\hline fumaric ac. & $\begin{array}{l}\text { Rhizopus nigricans } \\
\text { Ehrlich, I9I } 2\end{array}$ \\
\hline gluconic ac. & $\begin{array}{l}\text { Asp. niger } \\
\quad \text { Bernhauer, } 1928\end{array}$ \\
\hline lactic ac. & $\begin{array}{l}\text { Lactobacillus leichmani } \\
\text { Fred, Peterson and Stiles, } \\
\text { I } 925\end{array}$ \\
\hline d-lactic ac. & $\begin{array}{l}\text { Clostridium thermocellum } \\
\text { Peterson, Fred and Martin, } \\
\text { 1926 }\end{array}$ \\
\hline oxalic ac. & $\begin{array}{l}\text { B. aceti, B. oxydans, B. xylinum } \\
\text { Banning, } 1902\end{array}$ \\
\hline \multicolumn{2}{|l|}{$\begin{array}{l}\text { MALIC AC. } \\
\text { acetaldehyde }\end{array}$} \\
\hline acetaldehyde & $\begin{array}{l}\text { B. coli } \\
\text { Nagai, 1923 }\end{array}$ \\
\hline $\begin{array}{l}\text { acetic ac., ethyl alc., succinic } \\
\text { ac. }\end{array}$ & $\begin{array}{l}\text { B. coli communis } \\
\text { Grey, } 19^{2} 3\end{array}$ \\
\hline acetic ac., lactic ac. & $\begin{array}{l}\text { Lactobacillus pentoaceticus } \\
\text { Peterson and Fred, } 1920\end{array}$ \\
\hline acetic ac., succinic ac. & $\begin{array}{l}\text { B. lactis aerogenes } \\
\text { Emmerling, I } 899\end{array}$ \\
\hline fumaric ac. & $\begin{array}{l}\text { B. coli communis } \\
\text { Quastel and Whetham, } 19^{2} 4\end{array}$ \\
\hline lactic ac. & $\begin{array}{l}\text { yeast } \\
\text { Lebedev and Russ, I916 }\end{array}$ \\
\hline oxalic ac. & $\begin{array}{l}\text { Asp. niger } \\
\quad \text { Raistrick and Clark, I } 919\end{array}$ \\
\hline pyruvic ac. & $\begin{array}{l}\text { B. Auorescens } \\
\text { Bejerinck and Folpmers, I9I6 }\end{array}$ \\
\hline MaLONIC AC. & $\begin{array}{l}\text { B. coli communis } \\
\text { Grey, } 19^{2} 3\end{array}$ \\
\hline oxalic ac. & $\begin{array}{l}\text { B. aceti, } B . \text { acetosum } \\
B . \text { ascendens, } B . \text { Kiitzingianum } \\
\text { B. Pasteurianum }\end{array}$ \\
\hline
\end{tabular}


Substrates

Products

MaLic AC. (cont.)

Maltose

acetaldehyde

acetic ac., acetylmethyl carbinol, $\mathrm{CO}_{2}$, ethyl alc., $\mathrm{H}_{2}$, lactic ac., succinic ac.

acetic ac., butyl alc., butyric ac., $\mathrm{CO}_{2}$, ethyl alc., $\mathrm{H}_{2}$, lactic ac.

acetone, ethyl alc., formic ac.

acetone, ethyl alc., pyruvic ac.

acetylmethyl carbinol

citric ac., oxalic ac.

Mannitol

acetic ac., $\mathrm{CO}_{2}$, ethyl alc., formic ac., lactic ac., succinic ac.

acetic ac., acetone, butyl alc., butyric ac., lactic ac.

acetic ac., acetylmethyl carbinol, $\mathrm{CO}_{2}$, ethyl alc., $\mathrm{H}_{2}$, lactic ac., succinic ac.

acetic ac., acetylmethyl carbinol, ethyl alc., $\mathrm{CO}_{2}$, formic ac, lactic ac., succinic ac.

acetic ac., butyl alc., butyric ac., $\mathrm{CO}_{2}$, ethyl alc., $\mathrm{H}_{2}$, lactic ac.
Microorganisms

Authors

Termobacterium aceti

Banning, 1902

Bact. coli, B. lactis aerogenes

Nagai, 1923

B. tartricus

Grimbert, I90 I

Amylobacter butylicus

Duclaux, I 895

B. acetoethylicum

Northrop, Ashe and Morgan, I9I9

B. acetoethylicum

Speakman, 1925

$B$. anthracis

Lemoigne, I9I9

Asp. niger

Amelung, 1927

B. cloacae, B. coli,

$B$. lactis aerogenes

Thompson, I9 2

B. granulobacter pectinovorum

Speakman, 1923

B. tartricus

Grimbert, I90 I

B. lactis aerogenes

Kay, I926

Amylobacter butylicus

Duclaux, I 895 


\begin{tabular}{|c|c|}
\hline $\begin{array}{l}\text { Substrates } \\
\text { Products }\end{array}$ & $\begin{array}{c}\text { Microorganisms } \\
\text { Authors }\end{array}$ \\
\hline \multicolumn{2}{|l|}{ Mannitol (cont.) } \\
\hline acetic ac., $\mathrm{CO}_{2}$, ethyl alc., $\mathrm{H}_{2}$ & $\begin{array}{l}\text { B. ethaceticus } \\
\text { Frankland and Lumsden, } 189^{2}\end{array}$ \\
\hline $\begin{array}{l}\text { acetic ac., } \mathrm{CO}_{2} \text {, ethyl alc., } \mathrm{H}_{2} \text {, } \\
\text { lactic ac., succinic ac. }\end{array}$ & $\begin{array}{l}\text { B. coli communis } \\
\text { Grey, I9I } 8\end{array}$ \\
\hline $\begin{array}{l}\text { acetic ac., } \mathrm{CO}_{2} \text {, ethyl alc., } \mathrm{H}_{2} \text {, } \\
\text { lactic ac., succinic ac. }\end{array}$ & $\begin{array}{l}\text { B. coli communis } \\
\text { Harden, I } 901\end{array}$ \\
\hline $\begin{array}{l}\text { acetic ac., } \mathrm{CO}_{2} \text {, ethyl alc., } \mathrm{H}_{2} \text {, } \\
\text { succinic ac. }\end{array}$ & $\begin{array}{l}\text { B. ethacetosuccinicus } \\
\text { Frankland and Frew, I } 89^{2}\end{array}$ \\
\hline acetic ac., ethyl alc. & $\begin{array}{l}\text { B. ethaceticus } \\
\text { Frankland, Stanley and Frew, } \\
\text { I } 89 \mathrm{I}\end{array}$ \\
\hline acetic ac., ethyl alc. & $\begin{array}{l}\text { Friedlander's pneumococcus } \\
\text { Frankland, Stanley and Frew, } \\
\text { I } 89 \mathrm{I}\end{array}$ \\
\hline $\begin{array}{l}\text { acetic ac., } \mathrm{CO}_{2} \text {, ethyl alc., } \\
\text { formic ac., lactic ac., suc- } \\
\text { cinic ac., }\end{array}$ & $\begin{array}{l}\text { B. coli communis } \\
\text { Kay, } 1926\end{array}$ \\
\hline acetic ac., ethyl alc., formic ac., & B. ethaceticus \\
\hline succinic ac. & Frankland and Fox, I 889 \\
\hline acetic ac., ethyl alc., 1-lactic ac. & $\begin{array}{l}\text { Friedlander's pneumobacillus } \\
\text { Grimbert, } 1896\end{array}$ \\
\hline acetic ac., lactic ac. & $\begin{array}{l}\text { Lactobaiillus pentoaceticus } \\
\text { Peterson and Fred, I920 }\end{array}$ \\
\hline acetylmethyl carbinol & B. subtilis \\
\hline & $\begin{array}{l}\text { Tyrothrix tenuis } \\
\text { B. mesentericus vulgatus } \\
\text { Harden and Norris, } 19 \text { I } 2\end{array}$ \\
\hline $\begin{array}{l}\text { acetylmethyl carbinol, 2, 3- } \\
\text { butylene glycol }\end{array}$ & $\begin{array}{l}\text { B. cloacae } \\
\text { Thompson, I912 }\end{array}$ \\
\hline $\begin{array}{l}\text { acetylmethyl carbinol, 2, 3- } \\
\text { butylene glycol }\end{array}$ & $\begin{array}{l}\text { B. lactis aerogenes } \\
\text { Harden and Norris, 1912 }\end{array}$ \\
\hline butyl alc. & $\begin{array}{l}\text { B. butylicus } \\
\text { Emmerling, I } 897\end{array}$ \\
\hline butyl alc., butyric ac., lactic ac. & $\begin{array}{l}\text { B. butylicus } \\
\text { Fitz, I } 882\end{array}$ \\
\hline
\end{tabular}




\begin{tabular}{|c|c|}
\hline $\begin{array}{l}\text { Substrates } \\
\text { Products }\end{array}$ & $\begin{array}{l}\text { Microorganisms } \\
\text { Authors }\end{array}$ \\
\hline \multicolumn{2}{|l|}{ Mannitol (cont.) } \\
\hline citric ac. & $\begin{array}{l}\text { Asp. fumaricus } \\
\text { Schreyer, } 1928\end{array}$ \\
\hline citric ac., oxalic ac. & $\begin{array}{l}\text { Asp. niger, Citromyces glaber } \\
\text { Butkewitsch, I923 }\end{array}$ \\
\hline ethyl alc. & $\begin{array}{l}\text { Asp. niger } \\
\text { Kostytschew and Afanass- } \\
\text { jewa, } 19^{22}\end{array}$ \\
\hline ethyl alc., $\mathrm{CO}_{2}$ & $\begin{array}{l}\text { yeast } \\
\text { Kostytschew and Faermann, } \\
\text { I } 928\end{array}$ \\
\hline lactic ac. & $\begin{array}{l}\text { B. coli } \\
\text { Péré, I } 898\end{array}$ \\
\hline levulose & $\begin{array}{l}\text { Acetobacter suboxydans } \\
\text { Kluyver and deLeeuw, } 1924\end{array}$ \\
\hline levulose & $\begin{array}{l}\text { Bact. aceti } \\
\text { Brown, } 1886\end{array}$ \\
\hline levulose & $\begin{array}{l}\text { B. xylinum } \\
\text { Hoyer, } 1898\end{array}$ \\
\hline levulose & $\begin{array}{r}\text { Sorbose bacterium } \\
\text { Bertrand, } 1898\end{array}$ \\
\hline oxalic ac. & $\begin{array}{l}\text { Asp. niger } \\
\text { Elfving, I919 }\end{array}$ \\
\hline oxalic ac. & $\begin{array}{l}\text { B. industrium } \\
\text { Banning, } 1902\end{array}$ \\
\hline \multirow{2}{*}{$\begin{array}{l}\text { Mannose } \\
\text { acetic ac., ethyl alc., lactic ac. }\end{array}$} & \\
\hline & $\begin{array}{l}\text { Lactobacillus pentoaceticus } \\
\text { Peterson and Fred, I } 920\end{array}$ \\
\hline acetone, ethyl alc., formic ac. & $\begin{array}{l}\text { B. acetoethylicum } \\
\text { Northrop, Ashe and Morgan, } \\
\text { I9I9 }\end{array}$ \\
\hline $\begin{array}{l}\text { acetylmethyl carbinol, 2, 3- } \\
\text { butylene glycol }\end{array}$ & $\begin{array}{l}\text { B. cloacae } \\
\text { Harden and Norris, } 1912\end{array}$ \\
\hline $\begin{array}{l}\text { acetylmethyl carbinol, 2, 3- } \\
\text { butylene glycol } \\
\text { citric ac. }\end{array}$ & $\begin{array}{l}\text { B. lactis aerogenes } \\
\text { Harden and Norris, I9I } 2 \\
\text { Citromyces, sp.? } \\
\text { Wehmer, } 1913\end{array}$ \\
\hline
\end{tabular}


Substrates

Products

Mannose (cont.)

ethyl alc.

gluconic ac.

lactic ac.

P-MeTHOXY-BENZALDEHYDE p-methoxy-benzoic ac., p-methoxy-benzyl alc.

Methyl acetol

See acetylmethyl carbinol

Methyl alc.

formic ac.

Methyl-benzoyl Carbinol + DEXTROSE

methyl-phenylethylene glycol

Methyl- $\alpha$-Chloroethyl Ketone methyl- $\alpha$-chloroethyl alc.

O-METHYL-CYCLOHEXANONE o-methyl cyclohexanol

d-l-METH YLETHYL-ACETALDEHYDE d-amyl alc., valeric ac.

Methylethylacetaldehyde i-amyl alc., i-valeric ac.

Methylethylketone + Sucrose d-methylethyl carbinol
Microorganisms

AUTHORS

yeast

Mezzadroli, I9 8

Asp. niger

Bernhauer, 1928

B. coli

Péré, 1898

yeast

Neuberg and Liebermann, I92 I

B. pyocyaneus

Supniewski, I923

yeast

Neuberg and Komarewsky, I 927

yeast

Santomauro, 1924

yeast

Akamatsu, 1923

B. pasteurianum

Neuberg and Simon, I926

B. ascendens

B. xylinum

Neuberg and Simon, I 926

yeast

Neuberg and Nord, I919 


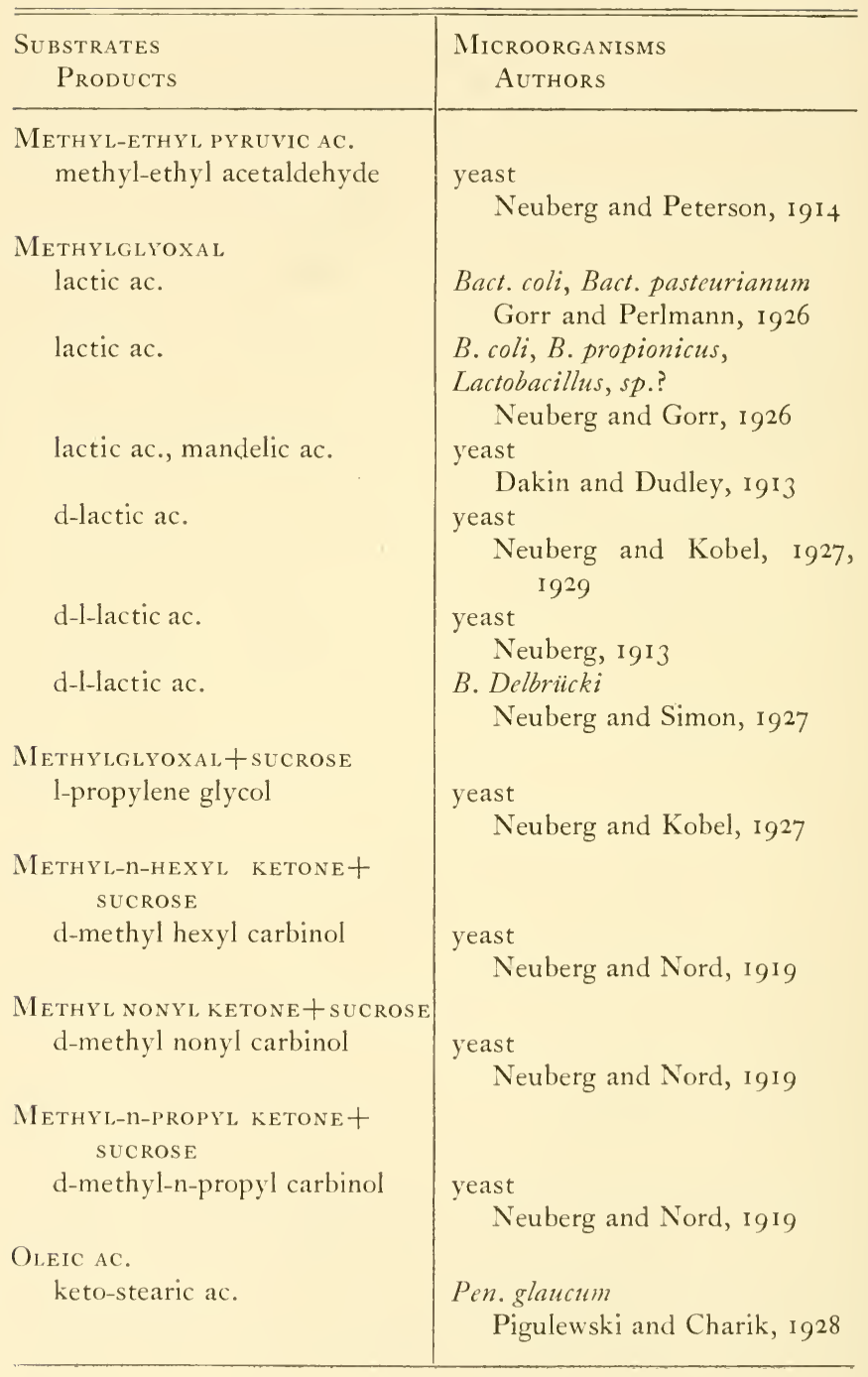




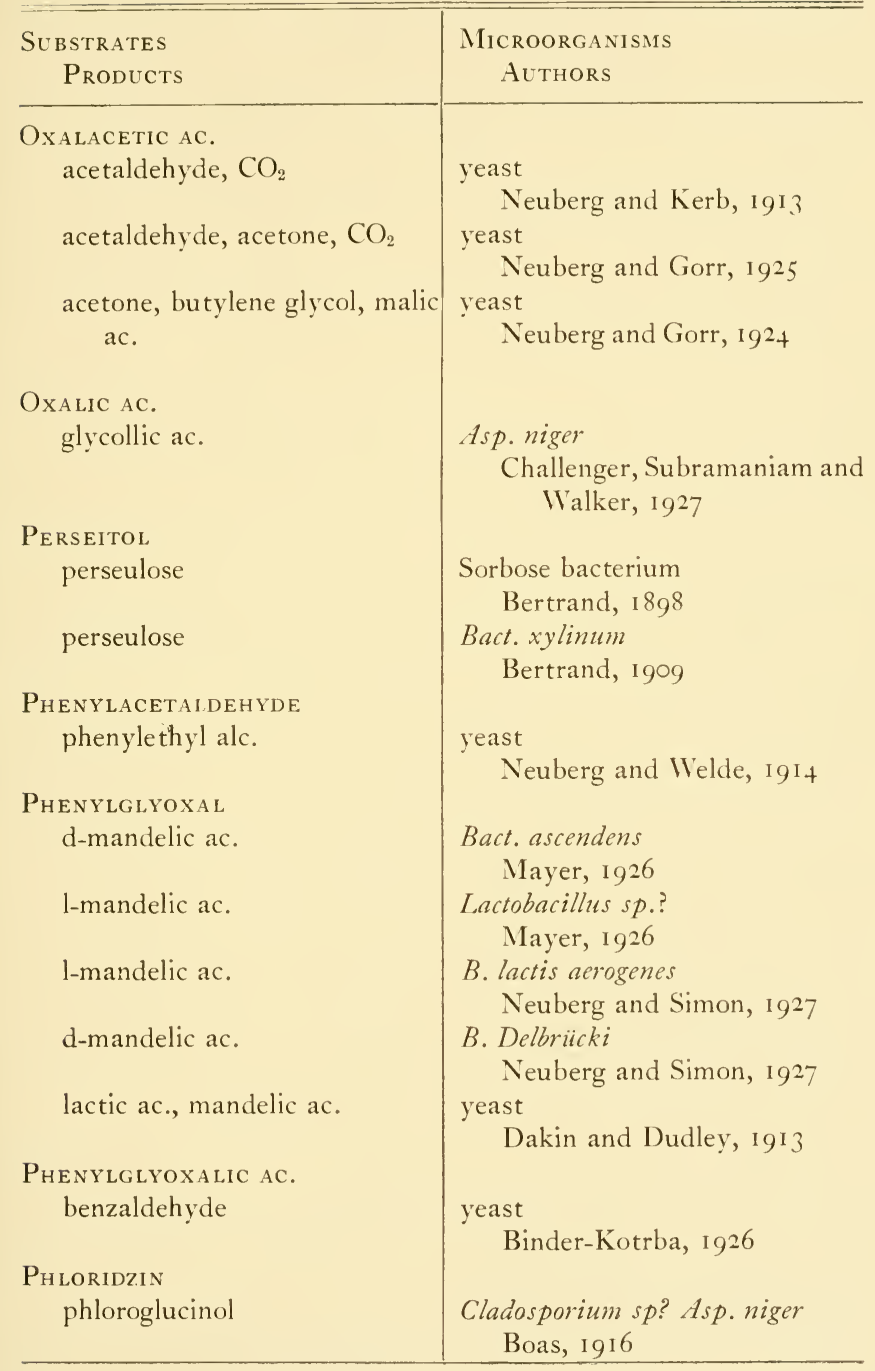


Substrates

Products
Microorganisms

Authors

Propionic ac.

butyl alc., butyric ac., caproic ac., caprylic alc.

\section{Propyl alc.}

propionic ac.

propionic ac.

propionic ac.

Propylene Glycol

acetic ac., $\mathrm{CO}_{2}$, propionic ac, succinic ac.

acetic ac., $\mathrm{CO}_{2}$, formic ac., glycollic ac., $\mathrm{H}_{2}$, i-lactic ac., propionic ac.

acetic ac., fumaric ac., lactic ac.

acetyl carbinol

acetylmethyl carbinol

acetylmethyl carbinol

aldol

ethyl alc.

lactic ac.

$\alpha$-PROPYLENE GLYCOL

acetyl carbinol

B. butylicus

Neuberg and Arinstein, 1921

Bact. aceti

Brown, 1886

B. Kützingianum

B. Pasteurianum

Mayer, 1898

Acetobacter, sp.?

$B$. aceti (Hansen)

Buchner and Gaunt, I 906

Propionibacterium Fensenii

Propionibacterium technicum

Van Niel, 1928

B. coli

Aubel, 1924

Rhizopus nigricans

Gottschalk, 1925

Mycoderma aceti

Kling, 1905

yeast

Neuberg and May, 1923

yeast

Hirsch, 1922

yeast

Neuberg, I9 12

yeast

Neuberg and Kerb, 1913

$B$. pyocyaneus

Aubel, 1924

Acetobacter xylinum

visser't Hooft, 1925 


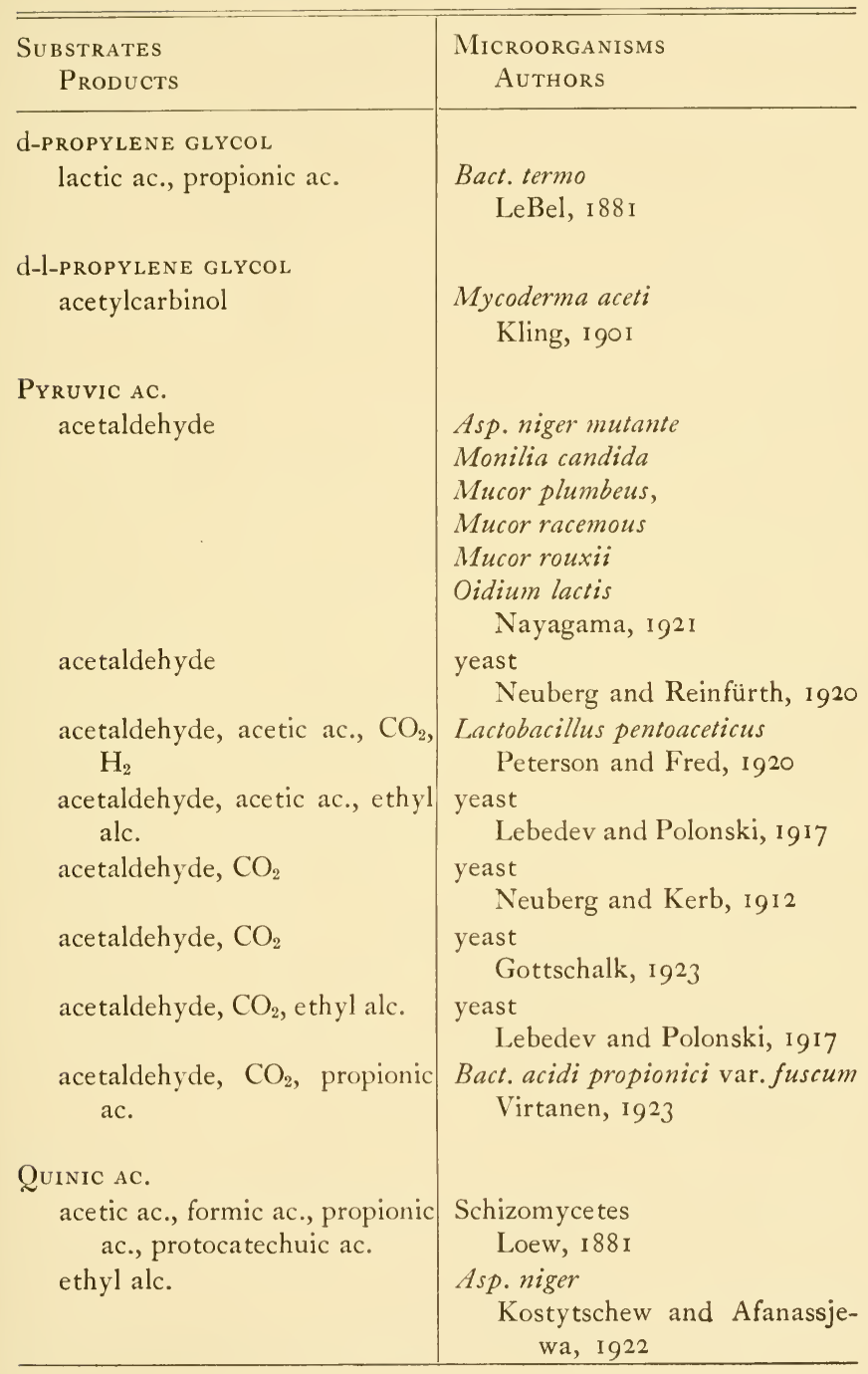




\begin{tabular}{l|l}
\hline $\begin{array}{c}\text { Substrates } \\
\text { Products }\end{array}$ & $\begin{array}{c}\text { Microorganisms } \\
\text { Authors }\end{array}$ \\
\hline $\begin{array}{c}\text { Quinic ac. (cont.) } \\
\text { protocatechuic ac. }\end{array}$ & Micrococcus chinicus \\
\hline
\end{tabular}

protocatechuic ac.

Rhamnose

acetylmethyl carbinol, 2, 3butylene glycol

\section{Raffinose}

acetone, ethyl alc., formic ac.

arabin

oxalic ac.

oxalic ac.

Saccharic ac.

acetic ac., ethyl alc., formic ac., lactic ac., succinic ac. acetic ac., acetylmethyl carbinol, $\mathrm{CO}_{2}$, ethyl alc., formic ac., lactic ac., succinic ac. citric ac.

oxalic ac.

SORBITOL sorbose

Emmerling and Abderhalden, 1903

Asp. niger, Asp. orvzae

Citromyces glaber

Butkewitsch, 1924

B. cloacae

$B$. lactis aerogenes

Harden and Norris, I 912

B. acetoethylicum

Northrop, Ashe, and Morgan, 1919

B. spongiosus

Ruhland, 1906

Asp. niger

Gillot, I 899

B. aceti, B. ascendens

B. xylinum

Banning, 1902

B. coli communis

Kay, 1926

$B$. lactis aerogenes

Kay, 1926

Asp. niger

Challenger, Subramaniam and Walker, 1927 Asp. niger

Butkewitsch, I923

Acet. suboxydans

Kluyver and deLeeuw, 1924 


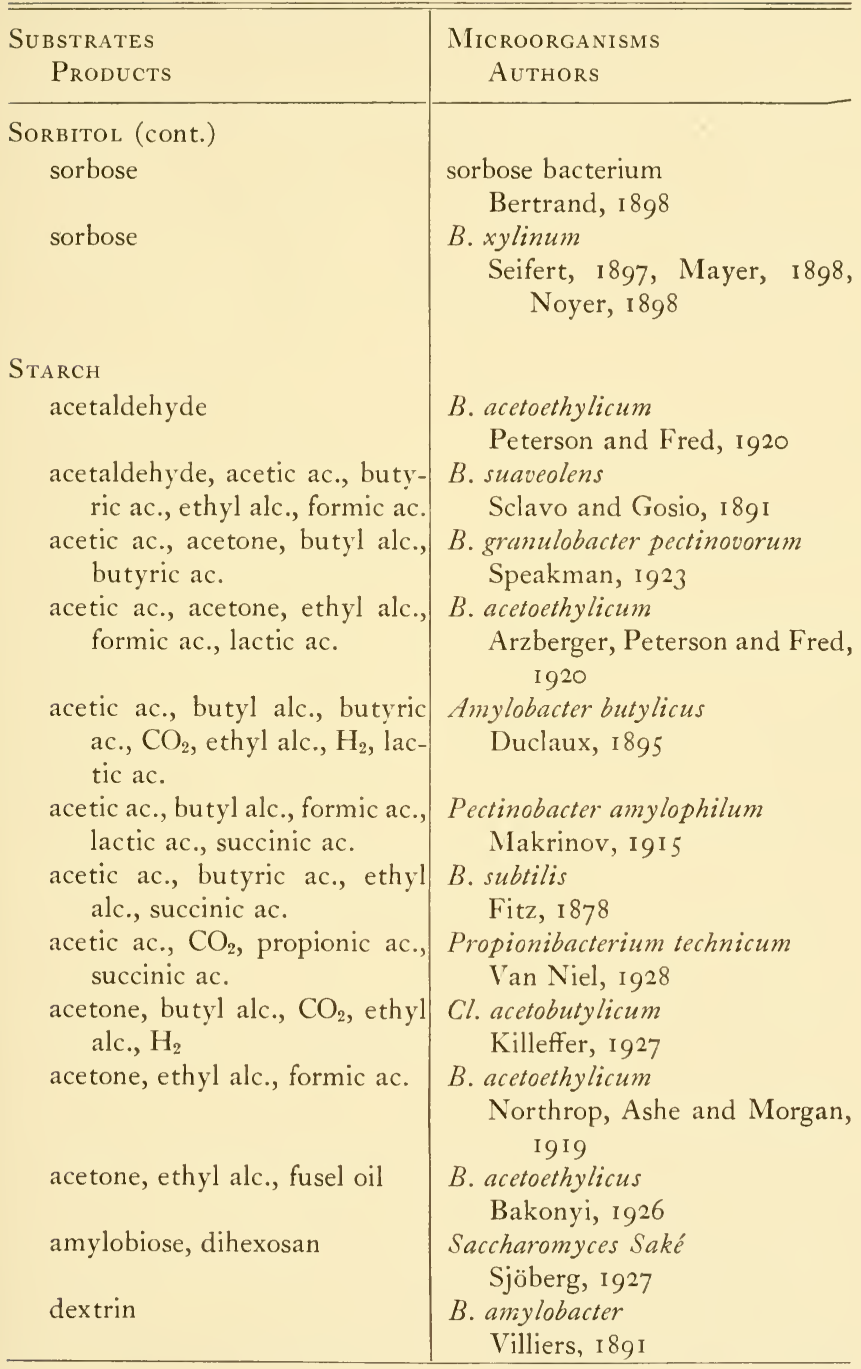




\begin{tabular}{l}
\hline $\begin{array}{l}\text { Substrates } \\
\text { Products }\end{array}$ \\
\hline Starch (cont.) \\
dextrose \\
ethyl alc. \\
ethyl alc., isobutyl alc., isopro- \\
pyl alc., n-butyl alc., propyl \\
alc. \\
maltose
\end{tabular}

oxalic ac.

Succinic ac.

acetic ac.

acetic ac., formic ac., $\mathrm{H}_{2}$

acetic ac., propionic ac.

fumaric ac.

oxalic ac.

propionic ac.

\section{Sucrose}

acetaldehyde

acetaldehyde

acetaldehyde, acetic ac., $\mathrm{CO}_{2}$, ethyl alc., glycerol

acetaldehyde, acetic ac., ethyl alc., glycerol

acetaldehyde, ethyl alc.
Microorganisms

AUTHORS

B. anthracis

Maumus, I 893

Mucor circinelloides (?)

Gayon and Dubourg, I 886

Granulobacterium butylicum

Folpmers, I921

Bact. thermoamylolyticus

Coolhaas, 1928

Asp. niger

Wehmer, I 89 I

Micrococcus casei liquefaciens

Orla-Jensen, I 904

B. coli communis

Grey, 1923

B. casei

Orla-Jensen, 1904

$B$. coli communis

Quastel and Whetham, I924 Asp. niger

Raistrick and Clark, I9I9

B. pyocyaneus

Quastel, 1924

yeast

Paris, 1922

$B$. coli, $B$. lactis aerogenes

Nagai, 1923

Sacc. Saké, Zygosacc. major,

Zygosacc, salsus

Kumagawa, 1922

yeast

Neuberg and Hirsch, I9I9 yeast

Gehle, 1922 


\begin{tabular}{|c|c|}
\hline $\begin{array}{l}\text { Substrates } \\
\text { Products }\end{array}$ & $\begin{array}{l}\text { Microorganisms } \\
\text { Authors }\end{array}$ \\
\hline \multicolumn{2}{|l|}{ Sucrose (cont.) } \\
\hline ehyde, ethyl alc. & $\begin{array}{l}\text { yeast } \\
\text { Zerner, } 1920\end{array}$ \\
\hline acetaldehyde, glycerol & $\begin{array}{l}\text { yeast } \\
\quad \text { Neuberg and Reinfurth, } 1918\end{array}$ \\
\hline $\begin{array}{l}\text { acetaldehyde, acetic ac., ethyl } \\
\text { alc., lactic ac. }\end{array}$ & $\begin{array}{l}\text { Amylobacter ethylicus } \\
\text { Duclaux, I } 895\end{array}$ \\
\hline acetic ac. & $\begin{array}{l}\text { Diplococcus pneumoniae } \\
\text { Brieger, } 1883\end{array}$ \\
\hline $\begin{array}{l}\text { acetic ac., acetone, ethyl alc. } \\
\text { lactic ac. }\end{array}$ & $\begin{array}{l}\text { B. invertenti-acetici } \\
\text { Mezzadroli, I9I7 }\end{array}$ \\
\hline $\begin{array}{c}\text { acetic ac., acetylmethylcarbi- } \\
\text { nol, 2, 3-butylene glycol, } \\
\mathrm{CO}_{2} \text {, ethyl alc., fo"mic ac., } \\
\mathrm{H}_{2} \text {, lactic ac., succinic ac. }\end{array}$ & $\begin{array}{l}\text { Aerobacter faeni } \\
\text { Breden, Fulmer, Werkman } \\
\text { and Hixon, } 1930\end{array}$ \\
\hline $\begin{array}{l}\text { acetic ac., butyl alc., butyric } \\
\text { ac., } \mathrm{CO}_{2} \text {, ethyl alc., } \mathrm{H}_{2} \text {, lac- } \\
\text { tic ac. }\end{array}$ & $\begin{array}{l}\text { Amylobacter butylicus } \\
\text { Duclaux, I } 895\end{array}$ \\
\hline c., lactic ac. & $\begin{array}{l}\text { B. invertenti-lattici } \\
\text { Mezzadroli, I9I7 }\end{array}$ \\
\hline acetic ac., malic ac., succinic ac. & $\begin{array}{l}\text { yeast } \\
\text { Kostytschew and Frey, I } 925\end{array}$ \\
\hline acetone, ethyl alc., fusel oi & $\begin{array}{l}\text { B. acetoethylicus } \\
\text { Bakonyi, I } 926\end{array}$ \\
\hline acetone, ethyl alc., fusel oil & yi, 1926 \\
\hline acetone, ethyl alc., f & $\begin{array}{l}\text { B. acetoethylicum } \\
\text { Northrop, Ashe and Morgan, } \\
\text { I919 }\end{array}$ \\
\hline acetylmethyl carbinol & $\begin{array}{l}\text { B. anthracis } \\
\text { Lemoigne, I } 919\end{array}$ \\
\hline acetylmethyl carbinol & $\begin{array}{l}\text { B. subtilis } \\
\text { Lemoigne, I9I3 }\end{array}$ \\
\hline acetylmethyl carbinol & $\begin{array}{l}\text { yeast } \\
\text { Neuberg and Simon, } 1925\end{array}$ \\
\hline $\begin{array}{l}\text { acetylmethyl carbinol, 2, 3-bu- } \\
\text { tylene glycol }\end{array}$ & $\begin{array}{l}\text { B. subtilis } \\
\text { Lemoigne, I912 }\end{array}$ \\
\hline
\end{tabular}


Substrates

Products

Sucrose (cont.)

arabin

butyl alc., butyric ac., lactic ac.

2, 3-butylene glycol

citric ac.

citric ac.

citric ac.

citric ac.

citric ac., fumaric ac., gluconic ac., malic ac.

citric ac., gluconic ac.

citric ac., gluconic ac., oxalic ac.

citric ac., gluconic ac., oxalic ac.

citric ac., gluconic ac., oxalic ac.

citric ac., oxalic ac.

citric ac., oxalic ac.

citric ac., oxalic ac.

ethyl alc.
Microorganisms

Authors

B. spongiosus

Ruhland, I906

B. butylicus

Fitz, I 882

yeast

Henninger, 1888

Asp. niger

Bernhauer, 1928

Citromyces, sp.?

Wehmer, I9I 3

Citromyces, sp.?

Filosofor and Malinovskii, I 928

Citromyces (Wehmer) .

Mazé, I 909

Asp. fumaricus

Wehmer, I9I 8, I928

Citromyces glaber

Pen. glaucum

Butkewitsch, 1927

Asp. niger

Bernhauer, 1924, 1926

Asp. niger

Butkewitsch, 1924

Sterigmatocystis nigra

Molliard, 1922

Asp. niger

Currie, I9I7

Asp. niger

Amelung, 1927

Asp. niger

Citromyces glaber

Butkewitsch, I923

Mucor racemosus

Fitz, I 876 


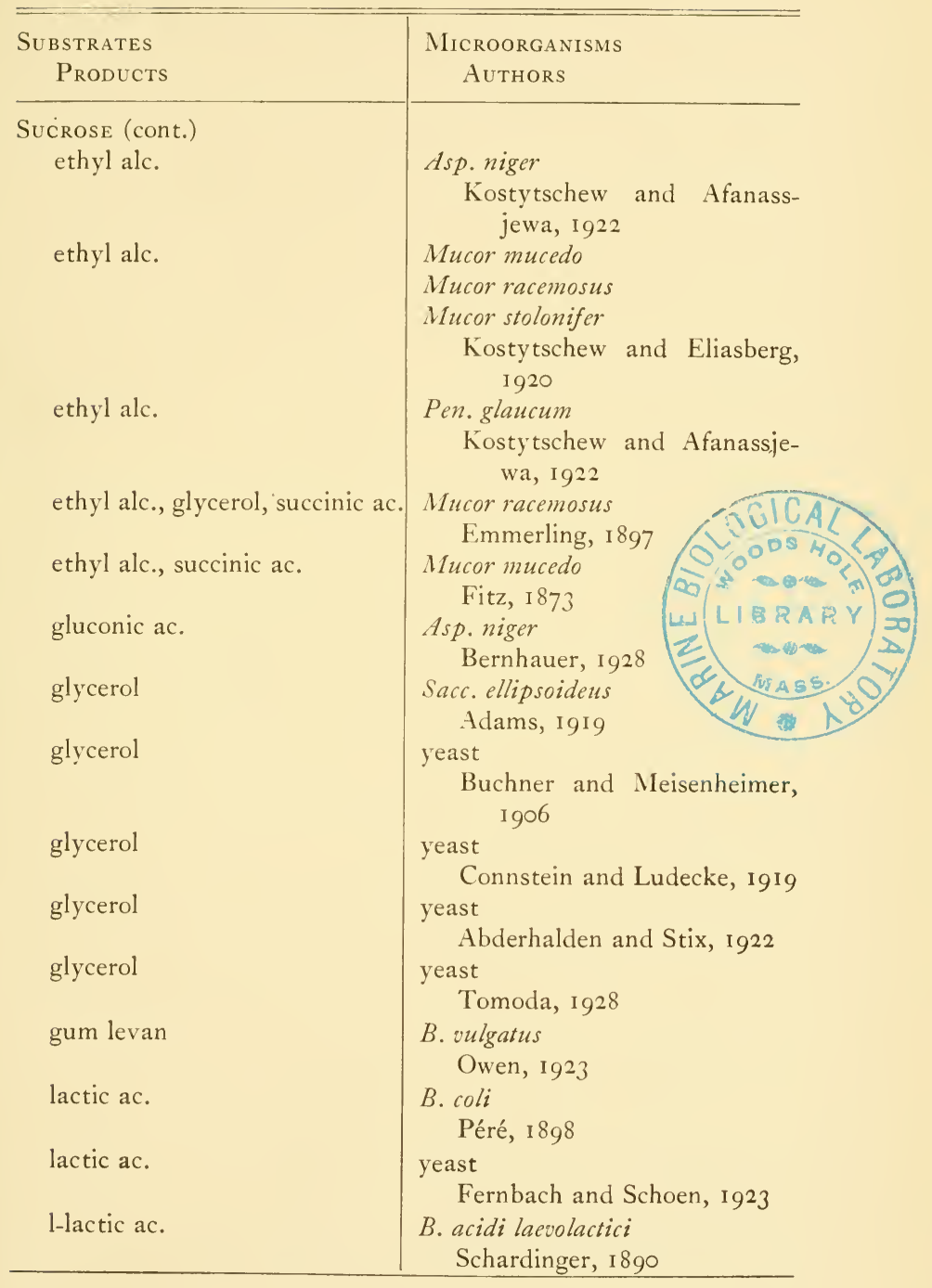


SubSTRATES

Products

Sucrose (cont.)

l-lactic ac.

d-lactic ac.

lactic ac., methylglyoxal

1-malic ac.

oxalic ac.

oxalic ac.

oxalic ac., citric ac.

pyruvic ac.

Sucrose + Methyl Glyoxal

1-propylene glycol

pyruvic ac.

TARTARIC Ac.

acetaldehyde

acetic ac., ethyl alc., succinic ac.

acetic ac., propionic ac., succinic ac.
Microorganisms

Authors

B. casei $E$.

Virtanen, Wichmann and

Lindström, I927

Strep. lactis

Virtanen, Wichmann and

B. coli

$$
\text { Lindström, I927 }
$$

Virtanen and Simola, I927 yeast

Dakin, I924

Asp. niger

Elfving, I 9 I 9

B. aceti

B. Pasteurianum

Banning, I902

Citromyces citricus

Citromyces glaber

Citromyces Pfefferianus

Penicillium glaucum

Butkewitsch, 1922

yeast

Klein and Fuchs, I929

yeast

Neuberg and Kobel, I927 yeast

Fernbach and Schoen, I9I3

B. coli

$B$. lactis aerogenes

Nagai, I923

B. coli communis

Grey, I923

Bact. termo

König, I 88 I 


\begin{tabular}{l} 
Substrates \\
Products \\
\hline
\end{tabular}

TARTARIC Ac. (cont.)

butyric ac., lactic ac., propionic ac., succinic ac. ethyl alc.

oxalic ac.

oxalic ac.

Thioacetaldehyde

ethylmercaptan

o-Tolyl Aldehyde

o-tolyl-acetyl carbinol

P-Tolyl Aldehyde

p-tolyl-acetyl carbinol

Trichlor Acetaldehyde

trichlorethylalcohol

$a, a, \beta$, Trichlorbutyl Aldehyde

2, 2, 3-trichlorbutanol

\section{VALEMITE}

keto-hexose

n-VALERALDEHYDE

n-amyl alc.

VAleraldehyde amyl alc.
Microorganisms

Authors

yeast

Karczag, I9 12

Asp. niger

Kostytschew and Afanassjewa, I9I 2

Asp. niger

Wehmer, I $89 \mathrm{I}$

Asp. niger

Raistrick and Clark, I9I9

yeast

Neuberg and Nord, I9I4

yeast

Behrens and Ivanoff, 1926

yeast

Behrens and Ivanoff, I926

yeast

Lintner and Lüers, I $9^{\text {I } 3}$

yeast

Rosenfeld, 1925

sorbose bacterium

Bertrand, 1898

yeast

Neuberg and Nord, I 9 I 4

yeast

Neuberg and Steenbock, I 9 I 4 


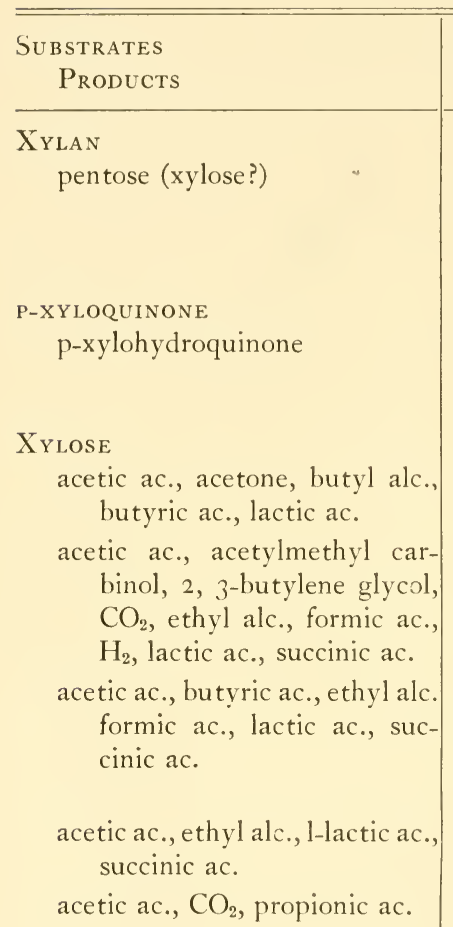

Microorganisms

Authors

Actinomyces sp.?

Patrick, Werkman and Hixon, I 930

coli bacteria

Neuberg and Simon, 1927

B. granulobacter pectinovorum

Speakman, I923

Aerobacter faeni

Breden, Fulmer, Werkman and Hixon, I930

$B$. lactis aerogenes

B. para typhoid

$B$ typhosus

Fred and Peterson, I920

Friedlander's pneumobacillus

Grimbert, I 896

Propionibacterium pentosaceum

Werkman, Hixon, Fulmer and Rayburn, I929

acetic ac., lactic ac.

acetic ac., lactic ac.

acetone, butyl alc., $\mathrm{CO}_{2}$
Lactobacillus pentosus

Lactobacillus pentoaceticus

Fred, Peterson and Anderson, I 92 I

Lactobacillus pentoaceticus

Fred, Peterson and Davenport, I9I9

B. granulobacter pectinovorum

Peterson, Fred and Schmidt, 1924 


\begin{tabular}{|c|c|}
\hline $\begin{array}{l}\text { Substrates } \\
\text { Products }\end{array}$ & $\begin{array}{c}\text { Microorganisms } \\
\text { AUthors }\end{array}$ \\
\hline \multirow{2}{*}{$\begin{array}{l}\mathrm{XYLOSE} \text { (cont.) } \\
\text { acetone, } \mathrm{CO}_{2} \text {, ethyl alc. }\end{array}$} & \\
\hline & $\begin{array}{l}\text { Acetobacter xylinum, } \\
\text { B. herbicola aureum, } \\
\text { B. vulgatus } \\
\text { Fred, Peterson and Anderson, } \\
\quad \text { I923 }\end{array}$ \\
\hline acetone, ethyl alc., formic ac. & $\begin{array}{l}\text { B. acetoethylicum } \\
\text { Northrop, Ashe and Morgan, } \\
\text { IgI9 }\end{array}$ \\
\hline citric ac. & $\begin{array}{l}\text { Citromyces, sp.? } \\
\text { Wehmer, I913 }\end{array}$ \\
\hline citric ac., oxalic ac. & $\begin{array}{l}\text { Asp. niger } \\
\text { Amelung, I927 }\end{array}$ \\
\hline $\begin{array}{l}\mathrm{CO}_{2} \text {, ethyl alc., glyceric alde- } \\
\text { hyde (?) } \\
\text { xylonic ac. }\end{array}$ & $\begin{array}{l}\text { Sacc. cerevisiae } \\
\text { Abbott, I } 926 \\
\text { sorbose bacterium } \\
\text { Bertrand, } 1898\end{array}$ \\
\hline
\end{tabular}



TABLE III

INDEX TO PRODUCTS 



\section{TABLE THREE \\ INDEX TO PRODUCTS}

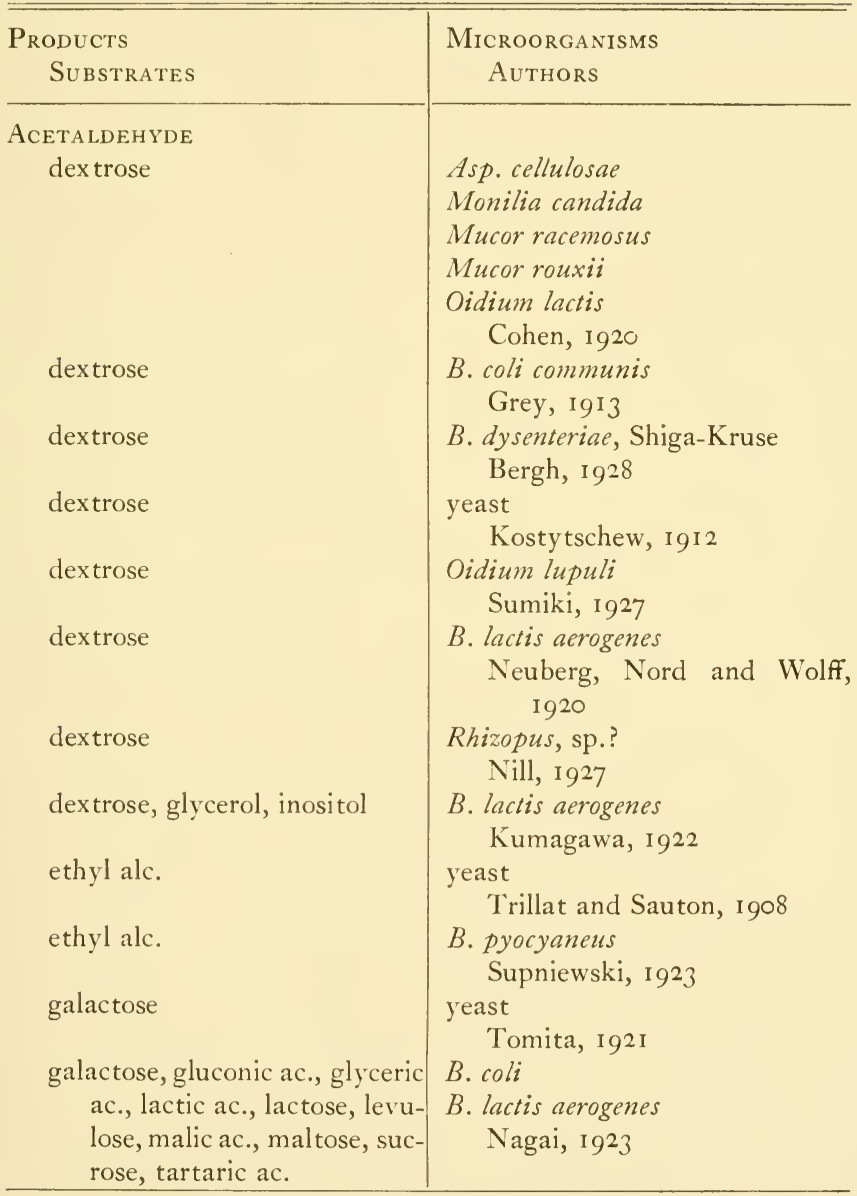




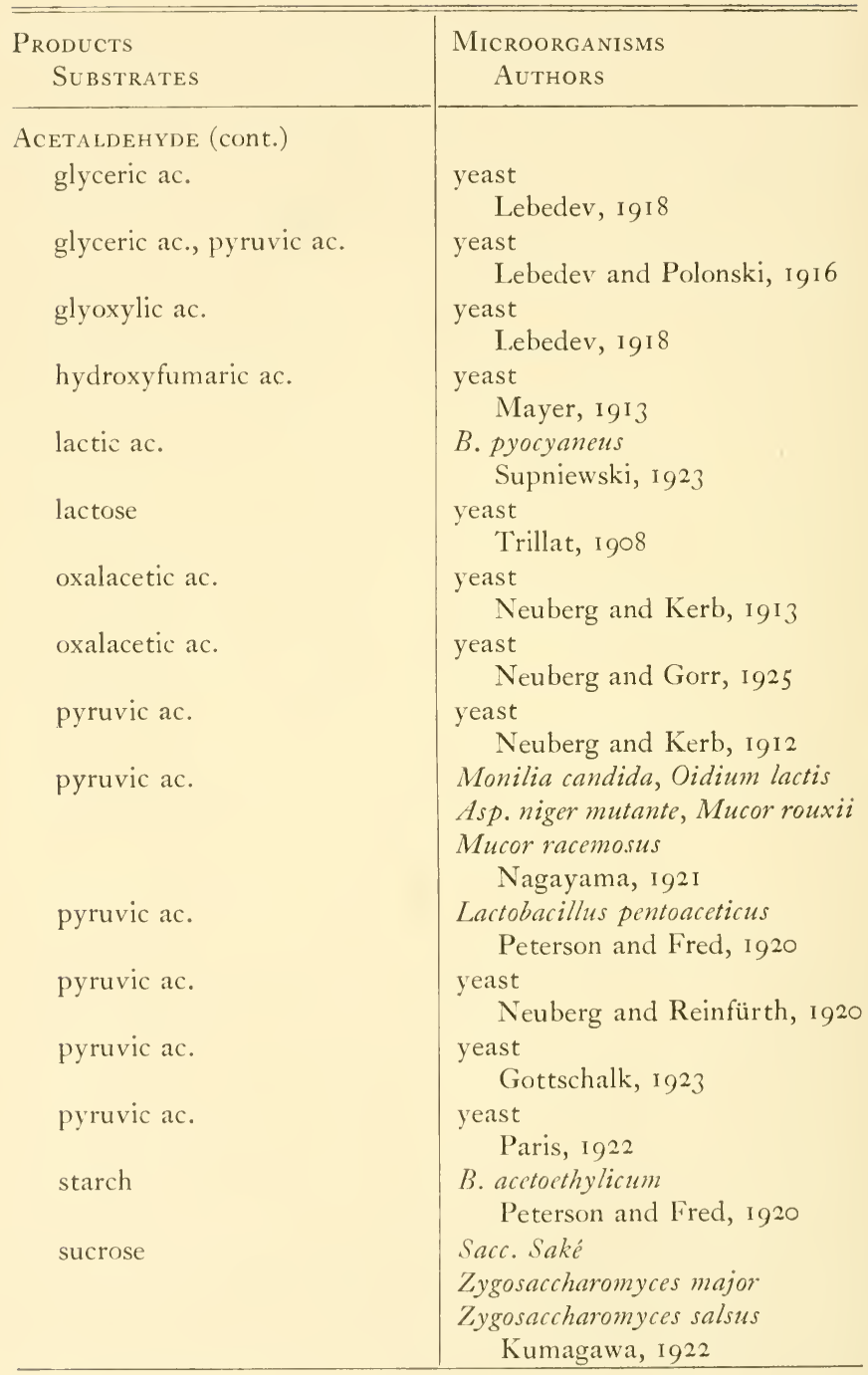




\begin{tabular}{|c|c|}
\hline $\begin{array}{l}\text { Products } \\
\text { Substrates }\end{array}$ & $\begin{array}{l}\text { Microorganisms } \\
\text { Authors }\end{array}$ \\
\hline \multicolumn{2}{|l|}{ ACETALDEHyde (cont.) } \\
\hline sucrose & $\begin{array}{c}\text { Amylobacter ethylicus } \\
\text { Duclaux, I } \$ 95\end{array}$ \\
\hline sucrose & $\begin{array}{l}\text { yeast } \\
\text { Gehle, } 1922\end{array}$ \\
\hline sucrose & yeast \\
\hline sucrose & $\begin{array}{l}\text { Neuberg and Reinfürth, I9I } 8 \\
\text { yeast } \\
\text { Zerner, I920 }\end{array}$ \\
\hline \multicolumn{2}{|l|}{ Асетіс AC. } \\
\hline acetaldehyde & $\begin{array}{l}\text { yeast } \\
\text { Kostytschew, } 19{ }^{1} 4\end{array}$ \\
\hline acetaldehyde & $\begin{array}{l}\text { B. Lactis aerogenes } \\
\text { Harden and Norris, I9I } 2\end{array}$ \\
\hline acetaldehyde & $\begin{array}{l}\text { B. pyocyaneus } \\
\text { Supniewski, } 1923\end{array}$ \\
\hline acetone & $\begin{array}{l}\text { B. pyocyaneus } \\
\text { Supniewski, } 1923\end{array}$ \\
\hline arabinose & $\begin{array}{l}\text { B. ethaceticum } \\
\text { Frankland and MacGregor, } \\
189^{2}\end{array}$ \\
\hline arabinose & $\begin{array}{l}\text { Lactobacillus arabinosus } \\
\text { Fred, Peterson and Anderson, } \\
\text { I } 9^{2} \text { I }\end{array}$ \\
\hline arabinose & $\begin{array}{l}\text { Pneumobacillus of Friedlander } \\
\text { Grimbert, } 1896\end{array}$ \\
\hline $\begin{array}{l}\text { arábinose, dextrose, galactose, } \\
\text { levulose, mannitol }\end{array}$ & $\begin{array}{l}\text { B. coli communis } \\
\text { Harden, I goI }\end{array}$ \\
\hline $\begin{array}{c}\text { arabinose, dextrose, galactose, } \\
\text { mannitol, starch, xylose }\end{array}$ & $\begin{array}{l}\text { B. granulobacter pectinovorum } \\
\text { Speakman, } 19^{2} 3\end{array}$ \\
\hline arabinose, xylose & Lactob. pentosus \\
\hline & $\begin{array}{l}\text { Lactob. pentoaceticus } \\
\text { Fred, Peterson and Anderson, } \\
\text { I } 92 \mathrm{I}\end{array}$ \\
\hline $\begin{array}{c}\text { arabinose, dextrose, glycerol, } \\
\text { lactic ac., starch, xylose }\end{array}$ & $\begin{array}{l}\text { Propionibacterium pentosaceum } \\
\text { Werkman, Hixon, Fulmer and } \\
\text { Rayburn, } 1929\end{array}$ \\
\hline
\end{tabular}




\begin{tabular}{|c|c|}
\hline $\begin{array}{l}\text { Products } \\
\text { Substrates }\end{array}$ & $\begin{array}{l}\text { Microorganisms } \\
\text { Authors }\end{array}$ \\
\hline \\
\hline cellulose & $\begin{array}{l}\text { Clostridium thermocellum } \\
\text { Viljoen, Fred and Peterson, } \\
\text { I926 }\end{array}$ \\
\hline cellulose & $\begin{array}{l}\text { B. cellulosae dissolvens } \\
\text { Khouvine, } 1923\end{array}$ \\
\hline cellulose & $\begin{array}{l}\text { Bact. cellaresolvens } \\
\text { Bact. opalescens } \\
\quad \text { Groenewege, } 1923\end{array}$ \\
\hline citric ac. & $\begin{array}{l}\text { B. coli communis } \\
\text { Grey, } 19^{2} 3\end{array}$ \\
\hline citric ac. & $\begin{array}{l}\text { B. suipestifer } \\
\text { Brown, Duncan and Henry, } \\
\text { I } 9^{24}\end{array}$ \\
\hline dextrose & $\begin{array}{l}\text { B. coli communis } \\
\text { Grey and Young, I92 I }\end{array}$ \\
\hline dextrose & $\begin{array}{l}\text { Granulobacter saccharobutyricum } \\
\text { Donker, } 1926\end{array}$ \\
\hline dextrose & $\begin{array}{l}\text { B. aerogenes } \\
\text { B. coli } \\
\quad \text { Virtanen and Simola, } 1927\end{array}$ \\
\hline dextrose & $\begin{array}{l}\text { Clostridium pasteurianum } \\
\text { Donker, } 1926\end{array}$ \\
\hline dextrose & $\begin{array}{l}\text { B. coli communis } \\
\text { Young, } 19^{2}+\end{array}$ \\
\hline dextrose & $\begin{array}{l}\text { B. acetoethylicum } \\
\text { Donker, I926 }\end{array}$ \\
\hline dextrose & $\begin{array}{l}\text { B. polymyxa } \\
\text { Donker, I } 926\end{array}$ \\
\hline dextrose & $\begin{array}{l}\text { Asp. niger } \\
\text { Heinze, } 1903\end{array}$ \\
\hline dextrose & $\begin{array}{l}\text { B. granulobacter pectinovorum, } \\
\text { Weizmann } \\
\text { Donker, } 1926\end{array}$ \\
\hline dextrose & $\begin{array}{l}\text { B. butylicus } \\
\text { Buchner and Meisenheimer, } \\
1908\end{array}$ \\
\hline
\end{tabular}




\begin{tabular}{|c|c|}
\hline $\begin{array}{l}\text { Products } \\
\text { Substrates }\end{array}$ & $\begin{array}{l}\text { Microorganisms } \\
\text { Authors }\end{array}$ \\
\hline \multicolumn{2}{|l|}{ Acetic AC. (cont.) } \\
\hline dextrose & $\begin{array}{l}\text { B. dysenteriae, Shiga-Kruse } \\
\text { Bergh, } 1929\end{array}$ \\
\hline dextrose & $\begin{array}{l}\text { B. coli communis } \\
\text { Grey, I } 918\end{array}$ \\
\hline dextrose & $\begin{array}{l}\text { B. typhosus } \\
\text { Harden, I } 901\end{array}$ \\
\hline dextrose & $\begin{array}{l}\text { B. typhosum } \\
\text { Scheffer, I } 928\end{array}$ \\
\hline dex trose & $\begin{array}{l}\text { B. coli } \\
\text { Scheffer, I } 928\end{array}$ \\
\hline dextrose & $\begin{array}{l}\text { B. dysenteriae, Shiga-Kruse } \\
\text { Scheffer, } 1928\end{array}$ \\
\hline dextrose & $\begin{array}{l}\text { B. aerogenes } \\
\text { Scheffer, I } 928\end{array}$ \\
\hline dextrose & $\begin{array}{l}\text { B. coli communis } \\
\text { Harden, I } 899\end{array}$ \\
\hline dextrose & $\begin{array}{l}\text { Oidium lupuli } \\
\quad \text { Sumiki, } 1927\end{array}$ \\
\hline dextrose & $\begin{array}{l}\text { B. coli } \\
\text { Grimbert, } 1896\end{array}$ \\
\hline dextrose & $\begin{array}{l}\text { Cl. Pasteurianum } \\
\text { Winogradsky, I902 }\end{array}$ \\
\hline dextrose & $\begin{array}{l}\text { Friedlander's pneumococcus } \\
\text { Frankland, Stanley and Frew, } \\
\text { I89I }\end{array}$ \\
\hline dextrose & $\begin{array}{l}\text { Serratia marcescens } \\
\quad \text { Pederson and Breed, } 1928\end{array}$ \\
\hline $\begin{array}{l}\text { dextrose, galactose, lactic ac., } \\
\text { mannose }\end{array}$ & $\begin{array}{l}\text { Lactobacillus pentoaceticus } \\
\text { Peterson and Fred, } 1920\end{array}$ \\
\hline $\begin{array}{l}\text { dextrose, gluconic ac., manni- } \\
\text { tol, saccharic ac. }\end{array}$ & $\begin{array}{l}\text { B. lactis aerogenes } \\
\text { Kay, } 19^{26}\end{array}$ \\
\hline dextrose, glycerol, lactic ac. & $\begin{array}{l}\text { Propionibacterium Freudenreichii } \\
\text { Propionibacterium Peterssonii } \\
\text { Propionibacterium Shermanii } \\
\text { Propionibacterium Thönii } \\
\quad \text { van Niel, I928 }\end{array}$ \\
\hline
\end{tabular}


Products

Substrate.s

Acetic AC (cont.)

dextrose, glycerol, lactic ac., pyruvic ac.

dextrose, glycerol, lactic ac.,

pyruvic ac., starch

dextrose, glycerol, lactic ac.

dextrose, lactic ac.

dextrose, lactose, maltose, mannitol, sucrose

dextrose, lactic ac., lactose, pyruvic ac.

dextrose, levulose

dextrose, mannitol

dextrose, mannitol

dextrose, mannitol

dextrose, mannitol

dextrose, gluconic ac., glycuronic ac., mannitol, saccha-

ric ac.

dextrose, starch, sucrose

dulcitol

dulcitol, mannitol

ethyl alc.
Microorganisms

AUthors

Propionibacterium Fensenii

van Niel, 1928

Propionibacterium technicum

van Niel, 1928

Propicnibacterium pentosaceum

van Niel, 1928

Propionibacterium rubrum

van Niel, I 928

B. tartricus

Grimbert, 1901

Bact. acidi propionici, var. fuscum

Virtanen, 1923

Bact. pyocyaneus

Aubel, I92 I

$B$. coli communis

Grey, 1918

B. ethaceticus

Frankland and Lumsden, 1892

B. cloacae

B. coli

$B$. lactis aerogenes

Thompson, I9I 2

$B$. lactis aerogenes

Harden and Walpole, I906

B. coli communis

Kay, 1926

$B$. acetoethylicum

Arzberger, Peterson and Fred, 1920

Pneumobacillus of Friedländer

Grimbert, I 896

$B$. ethacetosuccinicus

Frankland and Frew, $189^{2}$

Bact. aceti

Brown, 1886 


\begin{tabular}{|c|c|}
\hline $\begin{array}{l}\text { Products } \\
\text { Substrates }\end{array}$ & $\begin{array}{l}\text { Microorganisms } \\
\text { Authors }\end{array}$ \\
\hline \multicolumn{2}{|l|}{ ACETIC AC. (cont.) } \\
\hline ethyl alc. & $\begin{array}{l}\text { Acetobacter, sp.? } \\
\text { Knieriem and Mayer, I } 872 \\
\text { Buchner and Gaunt, I } 906 \\
\text { Day and Baker, I9I3 } \\
\text { Söhngen, I9I3 }\end{array}$ \\
\hline ethyl alc. & $\begin{array}{l}\text { Acetobacter suboxydans } \\
\text { visser't Hooft, } 1925\end{array}$ \\
\hline ethyl alc. & $\begin{array}{l}\text { Bact. aceti viscosum } \\
\text { Day and Baker, I9I } 3\end{array}$ \\
\hline ethyl alc. & $\begin{array}{l}\text { B. aceti (Hansen) } \\
\text { Buchner and Gaunt, I } 9 \text { I } 3\end{array}$ \\
\hline ethyl alc. & $\begin{array}{l}\text { B. Kützingianum } \\
\text { Mayer, I } 898\end{array}$ \\
\hline ethyl alc. & $\begin{array}{l}\text { B. Pasteurianum } \\
\text { Mayer, } 189^{8}\end{array}$ \\
\hline ethyl alc. & $\begin{array}{l}\text { B. pyocyaneus } \\
\text { Supniewski, } 1923\end{array}$ \\
\hline fumaric ac. & $\begin{array}{l}\text { B. pyocyaneus } \\
\text { Quastel, I924 }\end{array}$ \\
\hline glyceric ac. & $\begin{array}{l}\text { yeast } \\
\text { Lebedev, I9I } 8\end{array}$ \\
\hline glycerol & $\begin{array}{l}\text { B. coli communis } \\
\text { Grey, I923 }\end{array}$ \\
\hline glycerol & $\begin{array}{l}\text { Bact. aerogenes } \\
\text { Bact. coli } \\
\text { Bact. Freundii } \\
\quad \text { Braak, } 1928\end{array}$ \\
\hline glycerol & $\begin{array}{l}\text { B. lactis aerogenes } \\
\text { Harden and Norris, I } 912\end{array}$ \\
\hline glycerol, lactic ac. & $\begin{array}{l}\text { Amylobacter butylicus } \\
\text { Duclaux, I } 895\end{array}$ \\
\hline i-inositol & $\begin{array}{l}\text { B. lactis } \\
\text { Hewitt and Steabben, I92 I }\end{array}$ \\
\hline lactic ac. & $\begin{array}{l}\text { Acetobacter melanogenum } \\
\text { Acetobacter suboxydans }\end{array}$ \\
\hline
\end{tabular}




\begin{tabular}{l} 
Products \\
Substrates \\
\hline
\end{tabular}

Acetic Ac. (cont.)

lactic ac.

lactic ac.

lactic ac.

lactic ac.

lactose

lactose

lactose

lactose, maltose, mannitol, starch, sucrose levulose

malic ac.

malic ac.

malic ac.

malonic ac.

mannitol
Microorganisms

AUTHORS

Acetobacter xylinum

Visser't Hooft, I925

$B$. pyocyaneus

Supniewski, 1923

$B$. acidi propionici

$B$. acidi propionici-b

$B$. acidi propionici-a

von Freudenreich and Jensen, I 906

B. ethacetosuccinicus

Mazé, I9I3

B. casei

B. mobilis

Micrococcus casei liquefaciens

Orla-Jensen, 1904

Bact. acidi propionici

Sherman and Shaw, 1923

$B$ lactis aerogenes

Emmerling, I900

B. coli

Grimbert, I 896

Amylobacter butylicus

Duclaux, I 895

Lactobacillus pentoaceticus

Peterson and Fred, I920

B. coli communis

Grey, I923

$B$. lactis aerogenes

Fmmerling, I 899

Lactobacillus pentoaceticus

Peterson and Fred, I920

B. coli communis

Grey, 1923

Pneumobacillus of Friedlander

Grimbert, 1896 


\begin{tabular}{|c|c|}
\hline $\begin{array}{l}\text { Products } \\
\text { Substrates }\end{array}$ & $\begin{array}{l}\text { Microorganisms } \\
\text { Authors }\end{array}$ \\
\hline \multirow{2}{*}{$\begin{array}{l}\text { ACETIC AC. (cont.) } \\
\text { mannitol }\end{array}$} & \\
\hline & $\begin{array}{l}\text { Friedlander's pneumococcus } \\
\text { Frankland, Stanley and } \\
\text { Frew, I } 89 \mathrm{I}\end{array}$ \\
\hline mannitol & $\begin{array}{l}\text { Lactobacillus pentoaceticus } \\
\text { Peterson and Fred, I } 920\end{array}$ \\
\hline mannitol & $\begin{array}{l}\text { B. ethaceticus } \\
\text { Frankland, Stanley and Frew, } \\
\qquad \mathrm{I} 89 \mathrm{I}\end{array}$ \\
\hline mannitol, glycerol & $\begin{array}{l}\text { B. ethaceticus } \\
\text { Frankland and Fox, I } 889\end{array}$ \\
\hline pyruvic ac. & $\begin{array}{l}\text { Bact. coli } \\
\text { Aubel, I924 }\end{array}$ \\
\hline pyruvic ac. & $\begin{array}{l}\text { B. coli, B. proteus vulgatus } \\
\text { B. pyocyaneus } \\
\text { Cambier and Aubel, } 1922\end{array}$ \\
\hline pyruvic ac. & $\begin{array}{l}\text { Lactobacillus pentoaceticus } \\
\text { Peterson and Fred, I } 920\end{array}$ \\
\hline pyruvic ac. & $\begin{array}{l}\text { Rhizopus nigricans } \\
\text { Gottschalk, } 1925\end{array}$ \\
\hline pyruvic ac. & $\begin{array}{l}\text { yeast } \\
\text { Lebedev and Polonski, I9I7 }\end{array}$ \\
\hline quinic ac. & $\begin{array}{l}\text { Schizomycetes } \\
\text { Loew, I } 88 \text { I }\end{array}$ \\
\hline starch & $\begin{array}{l}\text { B. suaveolens } \\
\text { Sclavo and Gosio, I } 89 \mathrm{I}\end{array}$ \\
\hline starch & $\begin{array}{l}\text { B. subtilis } \\
\text { Fitz, I } 878\end{array}$ \\
\hline starch & $\begin{array}{l}\text { Pectinobacter amylophilum } \\
\text { Makrinov, I9I5 }\end{array}$ \\
\hline succinic ac. & $\begin{array}{l}\text { B. coli communis } \\
\text { Grey, } 19^{2} 3\end{array}$ \\
\hline succinic ac. & $\begin{array}{l}\text { B. casei } \\
\text { Micrococcus casei liquefaciens } \\
\quad \text { Orla-Jensen, I } 904\end{array}$ \\
\hline
\end{tabular}




\begin{tabular}{|c|c|}
\hline $\begin{array}{l}\text { Products } \\
\text { Substrates }\end{array}$ & $\begin{array}{l}\text { Microorganisms } \\
\text { AUTHORS }\end{array}$ \\
\hline \multicolumn{2}{|l|}{ Acetic AC. (cont.) } \\
\hline sucrose & $\begin{array}{l}\text { Aerobacter faeni } \\
\text { Breden, Fulmer, Werkman } \\
\text { and Hixon, I } 930\end{array}$ \\
\hline sucrose & $\begin{array}{l}\text { B. invertenti-lattici } \\
\text { B. invertenti-acetici } \\
\text { Mezzadroli, I9I7 }\end{array}$ \\
\hline sucrose & $\begin{array}{l}\text { Diplococcus pneumoniae } \\
\text { Brieger, I } 883\end{array}$ \\
\hline sucrose & $\begin{array}{l}\text { Amylobacter ethylicus } \\
\text { Duclaux, I } 895\end{array}$ \\
\hline sucrose & $\begin{array}{l}\text { Sacc. Saké, Zygosacc. major, } \\
\text { Zygosacc. salsus } \\
\quad \text { Kumagawa, } 1922\end{array}$ \\
\hline sucrose & $\begin{array}{l}\text { yeast } \\
\text { Kostytschew and Frey, I } 925\end{array}$ \\
\hline tartaric ac. & $\begin{array}{l}\text { B. coli communis } \\
\text { Grey, } 1923\end{array}$ \\
\hline tartaric ac. & $\begin{array}{l}\text { B. tartricus } \\
\text { Grimbert and Ficquet, I } 89^{8}\end{array}$ \\
\hline tartaric ac. & $\begin{array}{l}\text { Bact. termo } \\
\text { König, I } 88 \text { I }\end{array}$ \\
\hline xylose & $\begin{array}{l}\text { Pneumobacillus of Friedländer } \\
\text { Grimbert, I } 896\end{array}$ \\
\hline xylose & $\begin{array}{l}\text { Lactobacillus pentoaceticus } \\
\text { Fred, Peterson and Daven- } \\
\text { port, IgI9 }\end{array}$ \\
\hline xylose & $\begin{array}{l}\text { Aerobacter faeni } \\
\text { Breden, Fulmer, Werkman } \\
\text { and Hixon, I } 930\end{array}$ \\
\hline xylose & $\begin{array}{l}\text { B. lactis aerogenes, B. paratyphoid } \\
\text { B. typhosus } \\
\text { Fred and Peterson, I } 920\end{array}$ \\
\hline $\begin{array}{l}\text { ACETOACETIC AC. } \\
\text { butyric ac. }\end{array}$ & $\begin{array}{l}\text { Asp. niger } \\
\text { Coppock, Subramaniam and } \\
\text { Walker, I } 928\end{array}$ \\
\hline
\end{tabular}




\begin{tabular}{l} 
Products \\
Substrates \\
\hline
\end{tabular}

Acetoin

See acetylmethyl carbinol

Acetol

See acetyl carbinol

\section{Acetone}

arabinose, dextrin, dextrose, galactose, glycerol, lactic ac., levulose, maltose, mannose, raffinose, starch, sucrose, xylose

arabinose, dextrose, galactose, mannitol, starch, xylose arabinose, xylose

butyric ac.

2, 3-butylene glycol

citric ac.

dextrose

dextrose

dextrose

dextrose

dextrose, maltose
Microorganisms

Authors
B. acetoethylicum

Northrop, Ashe and Morgan 1919

B. granulobacter pectinovorum

Speakman, I923

B. granulobacter pectinovorum

Peterson, Fred and Schmidt, $19^{2} 4$

Asp. niger

Coppock, Subramaniam and Walker, 1928

Acetobacter suboxydans

Acetobacter xylinum

visser't Hooft, I925

Asp. niger

Challenger, Subramaniam and Walker, 1927

Cl. pasteurianum

Donker, 1926

B. granulobacter pectinovorum

Donker, 1926

B. acetoethylicum

Donker, 1926

B. polymyxa

Donker, 1926

$B$. acetoethylicum

Speakman, I925 


Products
Substrates

Acetone (cont.)

dextrose, starch, sucrose

isopropyl alc.

oxalacetic ac.

starch

starch, sucrose

sucrose

xylose

Acetone dicarboxylic ac. citric ac.

ACETYL CARBINOL

d-1-propylene glycol

Acetyl chlorbenzyl CARBinol chlorobenzaldehyde

Acetylmethyl Carbinol acetaldehyde, sucrose adonitol, mannitol arabinose, dextrose, galactose, isodulcite, mannose
Microorganisms

Authors

B. acetoethylicum

Arzberger, Peterson and Fred, 1920

Acetobacter xylinum

visser't Hooft, I 925

yeast

Neuberg and Gorr, 1925

Cl. acetobutylicum

Killeffer, 1927

$B$. acetoethylicus

B. macerans

Bakonyi, 1926

$B$. invertenti-acetici

Mezzadroli, I917

Acetobacter xylinum, B. vulgatus

Fred, Peterson and Anderson, 1923

Asp. niger

Walker, Subramaniam and Challenger, 1927

Mycoderma aceti

Kling, I90 I, I905

yeast

Neuberg and Liebermann, I92 I

yeast

Neuberg and Simon, 1925

$B$. lactis aerogenes

Harden and Norris, 1912

B. cloacae

$B$. lactis aerogenes

Harden and Norris, I9I 2 


\begin{tabular}{|c|c|}
\hline $\begin{array}{l}\text { Products } \\
\text { Substrates }\end{array}$ & $\begin{array}{l}\text { Microorganisms } \\
\text { Authors }\end{array}$ \\
\hline \multicolumn{2}{|l|}{ ACETYLMETHYL CARBinOL (cont.) } \\
\hline 2, 3-butylene glycol & $\begin{array}{l}\text { Mycoderma aceti } \\
\text { Kling, I } 905\end{array}$ \\
\hline dextrose & $\begin{array}{l}\text { B. lactis aerogenes } \\
\text { Neuberg, Nord and Wolff, } \\
1920\end{array}$ \\
\hline dextrose & $\begin{array}{l}\text { Serratia marcescens } \\
\quad \text { Pederson and Breed, } 1928\end{array}$ \\
\hline dextrose & $\begin{array}{l}\text { B. granulobacter pectinovorum, } \\
\text { Weizmann } \\
\text { Donker, } 1926\end{array}$ \\
\hline dextrose & $\begin{array}{r}\text { B. acetoethylicum } \\
\text { Donker, } 1926\end{array}$ \\
\hline dextrose & $\begin{array}{l}\text { B. polymyxa } \\
\text { Donker, I } 926\end{array}$ \\
\hline dextrose & $\begin{array}{l}\text { B. aerogenes } \\
\text { B. cloacae } \\
\quad \text { Scheffer, I } 928\end{array}$ \\
\hline dextrose, lactic ac. & $\begin{array}{c}\text { B. proteus, B. subtilis } \\
\text { Lemoigne, I } 9^{2} 3\end{array}$ \\
\hline $\begin{array}{l}\text { dextrose, lactose, maltose, } \\
\text { mannitol, sucrose }\end{array}$ & $\begin{array}{l}\text { B. tartricus } \\
\text { Grimbert, I } 90 \mathrm{I}\end{array}$ \\
\hline dextrose, levulose & $\begin{array}{l}\text { B. lactis aerogenes } \\
\text { Walpole, I } 9 \text { II }\end{array}$ \\
\hline dextrose, levulose & $\begin{array}{l}\text { Sarcina ventriculi } \\
\text { Smit, I } 928\end{array}$ \\
\hline dextrose, levulose & $\begin{array}{l}\text { Kluyver, Donker and Hooft } \\
\text { I } 925\end{array}$ \\
\hline $\begin{array}{l}\text { dextrose, levulose, sucrose, } \\
\text { acetaldehyde } \\
\text { dextrose, mannitol }\end{array}$ & $\begin{array}{l}\text { yeast } \\
\text { Neuberg and Reinfürth, I923 } \\
\text { B. cloacae } \\
\text { Thompson, I9I2 }\end{array}$ \\
\hline dextrose, mannitol & $\begin{array}{l}\text { B. lactis aerogenes } \\
\text { Harden and Walpole, I } 906\end{array}$ \\
\hline dextrose, mannitol & $\begin{array}{l}\text { B. mesentericus vulgatus } \\
B . \text { subtilis }\end{array}$ \\
\hline
\end{tabular}




\section{Products \\ Substrates \\ Acetylmethyl carbinol (cont.)}

dextrose, gluconic ac., mannitol, saccharic ac.

glycerol

levulose

maltose, sucrose

mannitol

oxalacetic ac.

pyruvic ac.

sucrose

sucrose, xylose

Acrolein

glycerol

glycerol

ACrylaldehyde

See acrolein

Adoninulose

adonitol

Aldol

pyruvic ac.
Microorganisms

AUthors

Tyrothrix tenuis

Harden and Norris, I9I 2

$B$. lactis aerogenes

Kay, 1926

Tyrothrix tenuis

Harden and Norris, I9I 2 yeast

Kluyver and Donker, 1924

B. anthracis

Lemoigne, I9I 9

B. mesentericus vulgatus

Harden and Norris, I9I 2 yeast

Neuberg and Gorr, 1924 yeast

Hirsch, 1922

B. subtilis

Lemoigne, I9I 3

Aerobacter faeni

Breden, Fulmer, Werkman and Hixon, 1930

B. Welchii

Humphreys, 1924

B. amaracrylus

Voisenet, I9I 8

Acetobacter suboxydans

$V$ isser't Hooft, I 925

yeast

Neuberg, I 912 


\begin{tabular}{l} 
Products \\
Substrates \\
\hline d-Amyl alc. \\
d-l-methylethyl acetaldehyde
\end{tabular}

i-AMYL ALC.

methylethylacetaldehyde

methylethyl pyruvic ac.

1-Amyl Alc.

methylethylacetaldehyde

n-Amyl alc.

glycerol

$a$-keto-n-caproic ac.

n-valeraldehyde

valeraldehyde

Amylobiose

starch

Arabin

raffinose, sucrose

Arabonic ac.

arabinose

Benzaldehyde

phenylglyoxalic ac.
Microorganisms

AUTHORS

B. pasteurianum

Neuberg and Simon, I926

B. xylinum

Neuberg and Simon, 1926

yeast

Neuberg and Peterson, I9I 4

B. ascendens

Neuberg and Simon, 1926

B. butylicus

Morin, I 887

yeast

Sen, 1923

yeast

Neuberg and Nord, I9I4 yeast

Neuberg and Steenbock, I9I4

Sacc. Saké

Sjöberg, 1927

B. spongiosus

Ruhland, I906

sorbose bacterium

Bertrand, I 898

yeast

Binder-Kotrba, 1926 


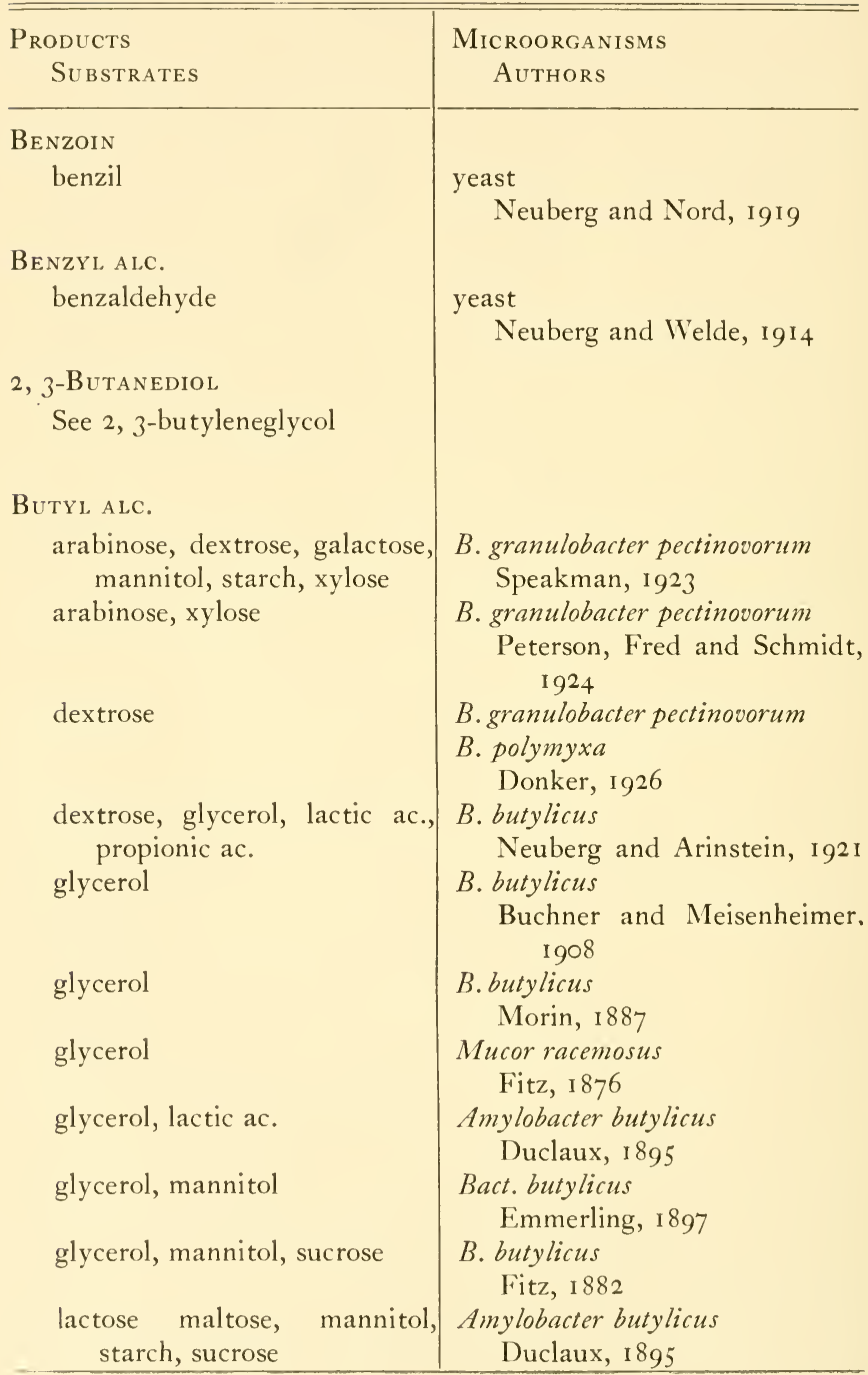




\begin{tabular}{|c|c|}
\hline $\begin{array}{l}\text { Products } \\
\text { Substrates }\end{array}$ & $\begin{array}{l}\text { Microorganisms } \\
\text { Authors }\end{array}$ \\
\hline \multicolumn{2}{|l|}{ Butyl alc. (cont.) } \\
\hline starch & $\begin{array}{l}\text { Cl. acetobutylicum } \\
\text { Killeffer, } 1927\end{array}$ \\
\hline starch & $\begin{array}{l}\text { Pectinobacter amylophilum } \\
\text { Makrinov, I9I } 5\end{array}$ \\
\hline starch & $\begin{array}{l}\text { Granulobacter butylicum } \\
\text { Folpmers, I } 921\end{array}$ \\
\hline \multicolumn{2}{|l|}{$\beta$-Butylene glycol } \\
\hline \multicolumn{2}{|l|}{ See I, 3-butylene glycol } \\
\hline \multicolumn{2}{|l|}{ I, 3-BUTYLENE GLYCOL } \\
\hline acetylmethyl carbinol & $\begin{array}{l}\text { yeast } \\
\text { Neuberg and Kobel, I925 }\end{array}$ \\
\hline \multicolumn{2}{|l|}{ 2, 3-BUTYLENE GLYCOL } \\
\hline acetaldehyde & $\begin{array}{l}\text { Bact. ascendens } \\
\text { Binder-Kotrba, I } 926\end{array}$ \\
\hline acetaldehyde & $\begin{array}{l}\text { B. lactis aerogenes } \\
\text { Harden and Norris, } 1912\end{array}$ \\
\hline acetaldol & $\begin{array}{l}\text { yeast } \\
\text { Neuberg and Kerb, I918 }\end{array}$ \\
\hline adonitol, mannitol & $\begin{array}{l}\text { B. lactis aerogenes } \\
\text { Harden and Norris, } 1912\end{array}$ \\
\hline $\begin{array}{l}\text { arabinose, dextrose, galactose, } \\
\text { isodulcite, mannose }\end{array}$ & $\begin{array}{l}\text { B. lactis aerogenes } \\
\text { Harden and Norris, } 1912\end{array}$ \\
\hline $\begin{array}{l}\text { arabinose, levulose, galactose, } \\
\text { isodulcite, mannose }\end{array}$ & $\begin{array}{l}\text { B. cloacae } \\
\text { Harden and Norris, I } 9 \text { I } 2\end{array}$ \\
\hline dextrose & $\begin{array}{l}\text { B. lactis aerogenes } \\
\text { Neuberg, Nord and Wolff, } \\
\text { I } 920\end{array}$ \\
\hline dextrose, levulose & $\begin{array}{l}\text { B. lactis aerogenes } \\
\text { Walpole, I9I I }\end{array}$ \\
\hline dextrose & $\begin{array}{l}\text { Serratia marcescens } \\
\quad \text { Pederson and Breed, } 1928\end{array}$ \\
\hline
\end{tabular}




Products
Substrates

2, 3,-ButYlene GLYCOL (cont.) dextrose

dextrose

dextrose, levulose

dextrose, mannitol

dextrose, mannitol

ethylene glycol, glycerol

glycerol

glycerol

lactic ac.

lactic ac.

levulose

oxalacetic ac.

sucrose

sucrose

sucrose, xylose
Microorganisms

AUTHORS

B. acetoethylicum

B. polymyxa

Cl. pasteurianum

Granulobacter saccharobutyricum

Donker, 1926

$B$. aerogenes, $B$. cloacae

Scheffer, I 928

yeast

Kluyver, Donker and Hooft, I925

B. cloacae

Thompson, I 9 I 2

$B$. lactis aerogenes

Harden and Walpole, I9o6

$B$. lactis aerogenes

Harden and Norris, I9I 2

Bact. aerogenes

Braak, I 928

$B$. lactis aerogenes

Harden and Norris, I 9 I 2

$B$. mesentericus

Lemoigne, I9I3

B. subtilis

Lemoigne, I923

yeast

Kluyver and Donker, I924 yeast

Neuberg and Gorr, I924

$B$. subtilis

Lemoigne, I9 12

yeast

Henninger, I 888

Aerobacter faeni

Breden, Fulmer, Werkman and Hixon, I930 


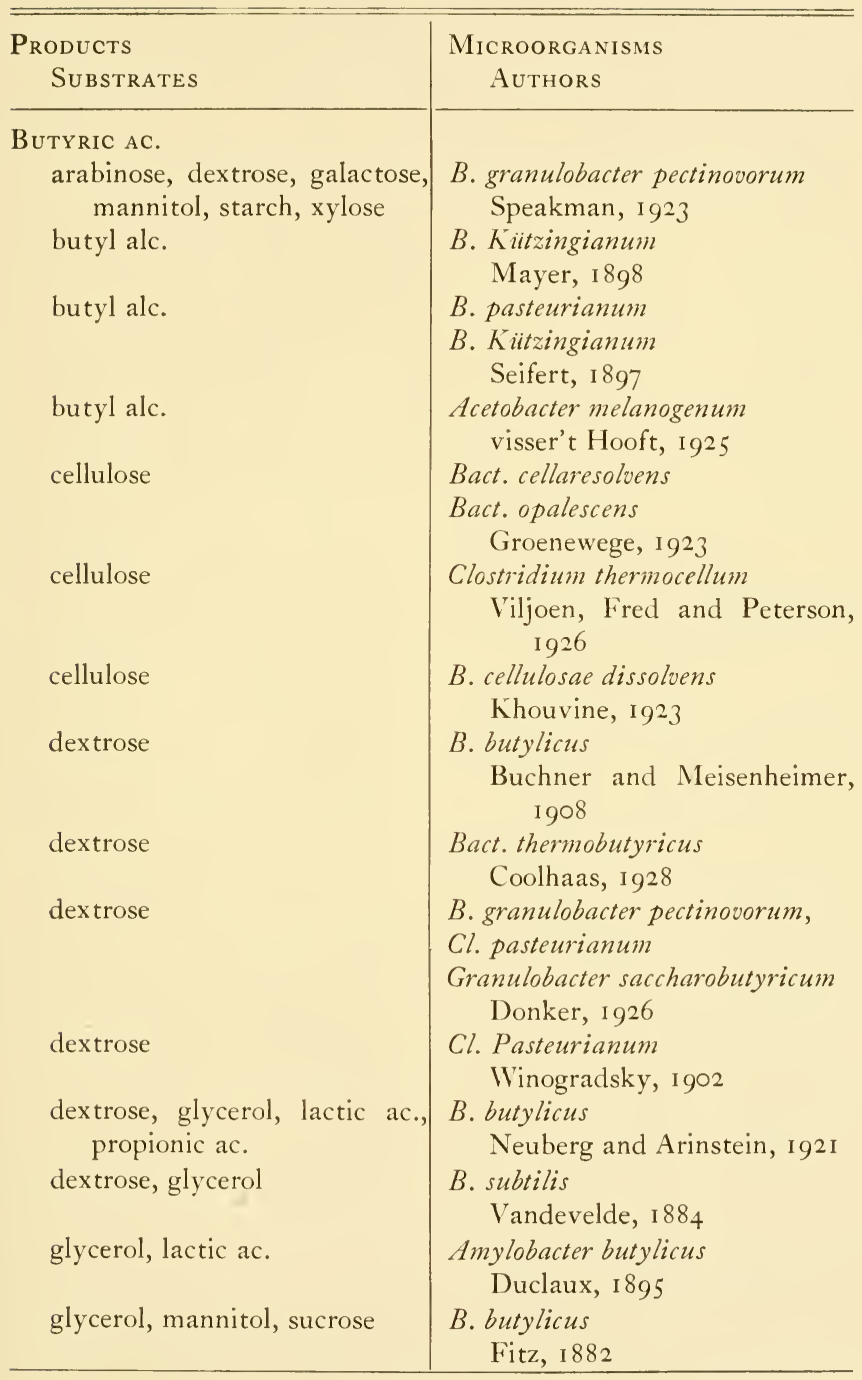




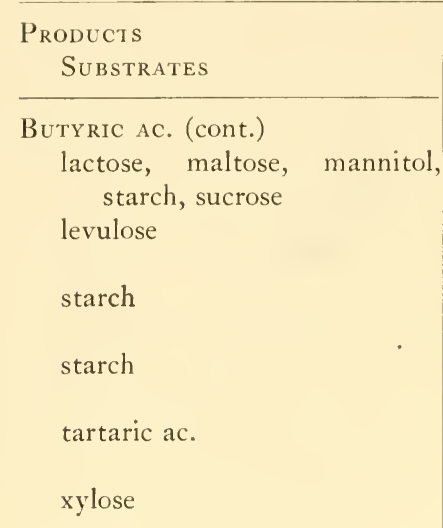

Caproic ac.

dextrose, glycerol, lactic ac., propionic ac.

Caprylic ac.

dextrose, glycerol, lactic ac., propionic ac.

Cellobiose cellulose

Cellulose

dextrose, levulose, mannitol

Chlorobenzoic ac. chlorobenzaldehyde

Chlorobenzyl alc. chlorobenzaldehyde
Microorganisms

Authors

Amylobacter butylicus

Duclaux, I 895

Sarcina maxima

Smit, 1928

B. suaveolens

Sclavo and Gosio, I89I

B. subtilis

Fitz, 1878

yeast

Karczag, I9I 2

B. paratyphoid

B. typhosus

Fred and Peterson, I920

B. butylicus

Neuberg and Arinstein, 1921

B. butylicus

Neuberg and Arinstein, I92 I

Bact. cellaresolvens

Bact. opalescens

Groenwege, 1923

Bact.xylinum

Brown, I 886

yeast

Neuberg and Liebermann, I92 I

yeast

Neuberg and Liebermann, I 92 I 


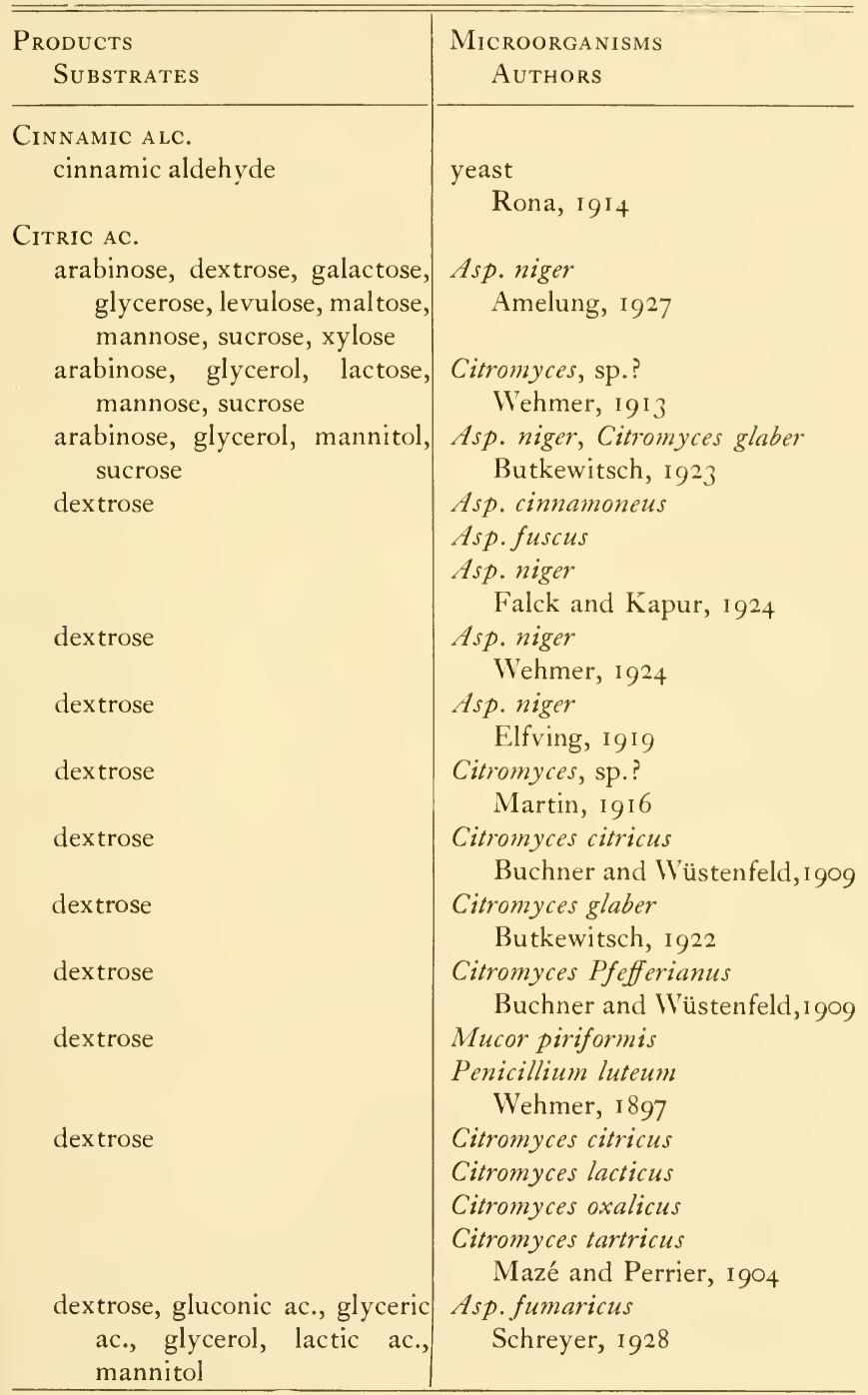




\begin{tabular}{|c|c|}
\hline $\begin{array}{l}\text { Products } \\
\text { SubSTRATES }\end{array}$ & $\begin{array}{l}\text { Microorganisms } \\
\text { Authors }\end{array}$ \\
\hline \multicolumn{2}{|l|}{ Citric ac. (cont.) } \\
\hline dextrose, sucrose & $\begin{array}{l}\text { Asp. niger } \\
\text { Bernhauer, I } 928\end{array}$ \\
\hline ethyl alc. & $\begin{array}{l}\text { Citromyces citricus } \\
\text { Citromyces tartricus } \\
\quad \text { Mazé and Perrier, } 1904\end{array}$ \\
\hline gluconic ac. & $\begin{array}{l}\text { Asp. niger } \\
\text { Walker, Subramaniam and } \\
\text { Challenger, } 1927\end{array}$ \\
\hline gluconic ac. & $\begin{array}{l}\text { Asp. fumaricus } \\
\text { Schreyer, I } 925\end{array}$ \\
\hline glycerol & $\begin{array}{l}\text { Citromyces citricus } \\
\text { Mazé and Perrier, I904 }\end{array}$ \\
\hline saccharic ac. & $\begin{array}{l}\text { Asp. niger } \\
\text { Challenger, Subramaniam and } \\
\text { Walker, } 1927\end{array}$ \\
\hline sucrose & $\begin{array}{l}\text { Asp. niger } \\
\text { Bernhauer, I } 926\end{array}$ \\
\hline sucrose & $\begin{array}{l}\text { Asp. niger } \\
\text { Butkewitsch, I924 }\end{array}$ \\
\hline sucrose & $\begin{array}{l}\text { Asp. niger } \\
\text { Bernhauer, I924 }\end{array}$ \\
\hline sucrose & $\begin{array}{l}\text { Asp. niger } \\
\text { Currie, I } 917\end{array}$ \\
\hline sucrose & $\begin{array}{l}\text { Citromyces, sp.? } \\
\text { Filosofor and Malinovski, } \\
\text { I } 928\end{array}$ \\
\hline sucrose & $\begin{array}{c}\text { Citromyces, sp.? } \\
\text { Mazé, Igog }\end{array}$ \\
\hline sucrose & $\begin{array}{l}\text { Asp. fumaricus } \\
\text { Wehmer, I9I } 8, \text { I } 928\end{array}$ \\
\hline sucrose & $\begin{array}{l}\text { Citromyces citricus } \\
\text { Citromyces glaber } \\
\text { Citromyces Pfefferianus } \\
\text { Penicillium glaucum } \\
\quad \text { Butkewitsch, I922 }\end{array}$ \\
\hline
\end{tabular}




\begin{tabular}{|c|c|}
\hline $\begin{array}{l}\text { Products } \\
\text { Substrates }\end{array}$ & $\begin{array}{l}\text { Microorganisms } \\
\text { Authors }\end{array}$ \\
\hline $\begin{array}{l}\text { Citric ac. (cont.) } \\
\text { sucrose } \\
\text { sucrose }\end{array}$ & $\begin{array}{l}\text { Citromyces glaber } \\
\text { Penicillium glaucum } \\
\text { Butkewitsch, 1927 } \\
\text { Sterigmatocystis nigra } \\
\text { Molliard, 1922 }\end{array}$ \\
\hline $\begin{array}{c}\text { Citronella Oil (or alc.) } \\
\text { a-citronellaldehyde }\end{array}$ & $\begin{array}{l}\text { yeast } \\
\text { Mayer and Neuberg, I9I } 5\end{array}$ \\
\hline $\begin{array}{l}\text { Dextrin } \\
\text { dihydroxyacetone } \\
\text { starch }\end{array}$ & $\begin{array}{l}\text { S. ludwigii } \\
\text { Neuberg and Kobel, I } 928 \\
\text { B. amylobacter } \\
\text { Villiers, I } 89 \mathrm{I}\end{array}$ \\
\hline $\begin{array}{l}\text { Dextrose } \\
\text { starch } \\
\text { phloridzin }\end{array}$ & $\begin{array}{l}\text { B. anthracis } \\
\quad \text { Naumus, I } 893 \\
\text { Asp. niger } \\
\text { Cladosporium, sp.? } \\
\quad \text { Boas, I9I6 }\end{array}$ \\
\hline $\begin{array}{l}a, a, \text { DICHLORPROPYL ALC. } \\
a, a \text {, dichloracetone } \\
\text { gluconic ac. }\end{array}$ & $\begin{array}{l}\text { yeast } \\
\text { Sen, I } 924 \\
\text { Acetobacter sp? } \\
\text { Söhngen, 1914, I915 }\end{array}$ \\
\hline $\begin{array}{l}\text { DiHyDRoXYACETONE } \\
\text { glycerol } \\
\text { glycerol } \\
\text { glycerol } \\
\text { glycerol }\end{array}$ & $\begin{array}{l}\text { Acetobacter suboxydans } \\
\text { Kluyver and deLeeuw, } 1924 \\
\text { sorbose bacterium } \\
\text { Bertrand, } 190^{2} \\
\text { B. xylinum } \\
\text { Bernhauer and Schön, } 1928 \\
\text { B. dioxyacetonicum } \\
\text { B. xylinum } \\
\quad \text { Virtanen and Barlund, } 1926\end{array}$ \\
\hline
\end{tabular}




\section{Products}

Substrates

DiHydRoxyACETONE (cont.) glycerol

glycerol

glycerol

ERYThrose

erythritol

ERYTHRULOSE

ery thritol

Ethyl alc.

acetaldehyde

arabinose

arabinose, dextrin, dextrose, galactose, glycerol, lactic ac., levulose, maltose, mannose, raffinose, starch, sucrose, xylose arabinose, dextrose, galactose, levulose, mannitol cellulose

cellulose

citric ac.

dextrin, starch
Microorganisms

Authors

Acetobacter suboxydans

Bact. xylinum

Brit. patent 269,950

Bact. xylinum

Bertrand and Sazerac, 190I sorbose bacterium

Bertrand, I 898

Acetobacter suboxydans

Kluyver and deLeeuw, 1924

Sorbose bacterium

Bertrand, I 898 , I 900

yeast

Kostytschew and Hübbenet, I 9 I 2

B. ethaceticus

Frankland and MacGregor, 1892

B. acetoethylicum

Northrop, Ashe and Morgan, I9I9

B. coli communis

Harden, I 901

Clostridium thermocellum

Viljoen, Fred and Peterson, I 926

B. cellulosae dissolvens

Khouvine, 1923

B. coli communis

Grey, 1923

Mucor circinelloides (?)

Gayon and Dubourg, I 886 


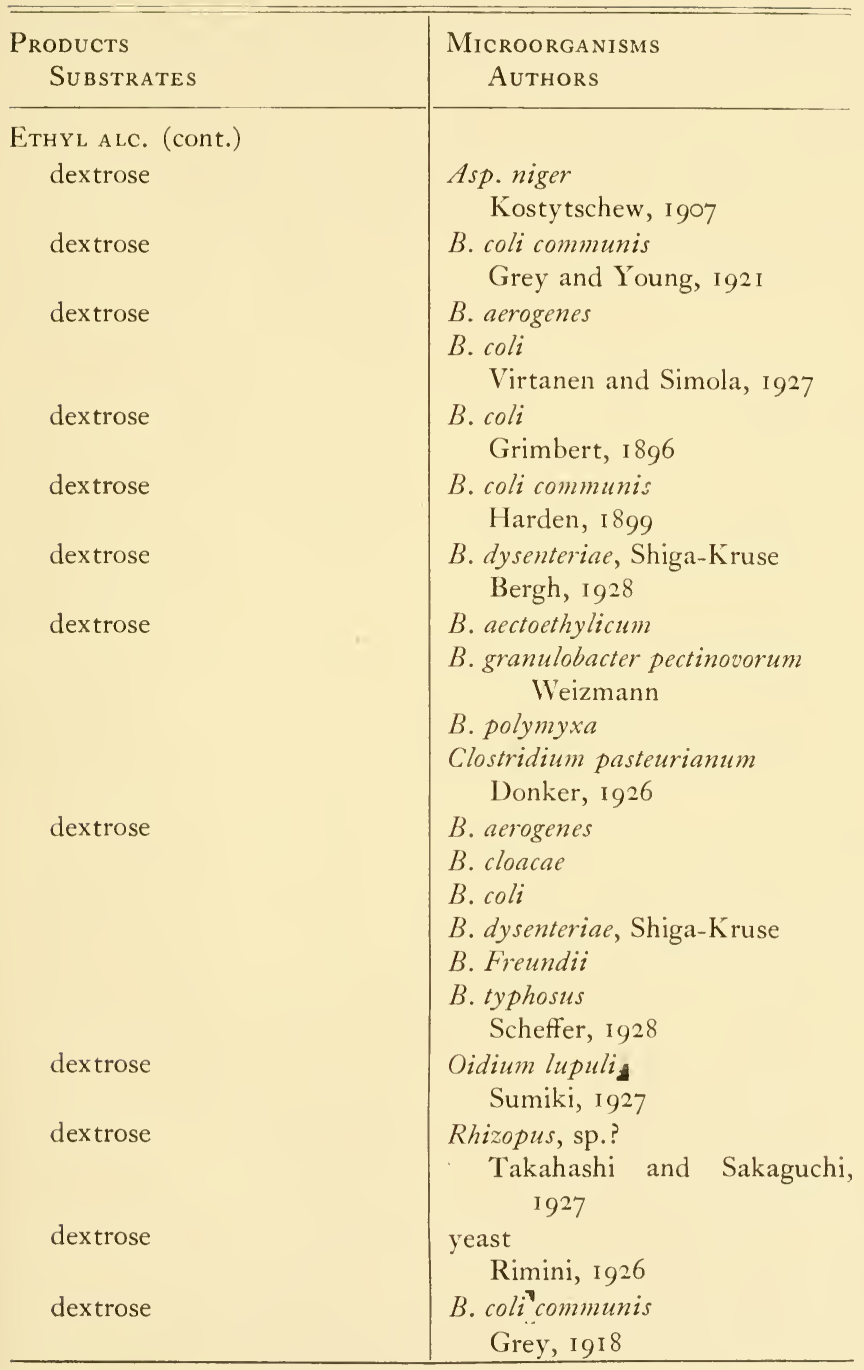




Products
Substrates

Ethyl alc. (cont.)

dextrose

dextrose

dextrose

dextrose

dextrose

dextrose

dextrose, galactose, mannose

dextrose, gluconic ac., glycerol, mannitol

dextrose, gluconic ac., mannitol

dextrose, lactose, maltose, mannitol, sucrose

dextrose, levulose

dextrose, levulose

dextrose, maltose

dextrose, mannitol

dextrose, mannitol

dextrose, mannitol
Microorganisms

Authors

$B$. of malignant oedema

Kerry and Frankel, i 890

Friedlander's pneumococcus

Frankland, Stanley and Frew, I $89 \mathrm{I}$

Rhizopus, sp.?

Nill, 1927

Fusarium lini

Anderson and Willaman, 1922

B. typhosus

Harden, I90 I

Serratia marcescens

Pederson and Breed, 1928

Lactobacillus pentoaceticus

Peterson and Fred, I920

B. coli communis

Kay, 1926

$B$. lactis aerogenes

Kay, I926

B. tartricus

Grimbert, I90 I

Bact. pyocyaneus

Aubel, I92 I

Sarcina maxima

Sarcina ventriculi

Smit, 1928

B. acetoethylicum

Speakman, I925

B. coli communis

Grey, I9 8

B. cloacae, B. coli

$B$. lactis aerogenes

Thompson, I9I 2

B. ethaceticus

Frankland and Lumsden, 1892 


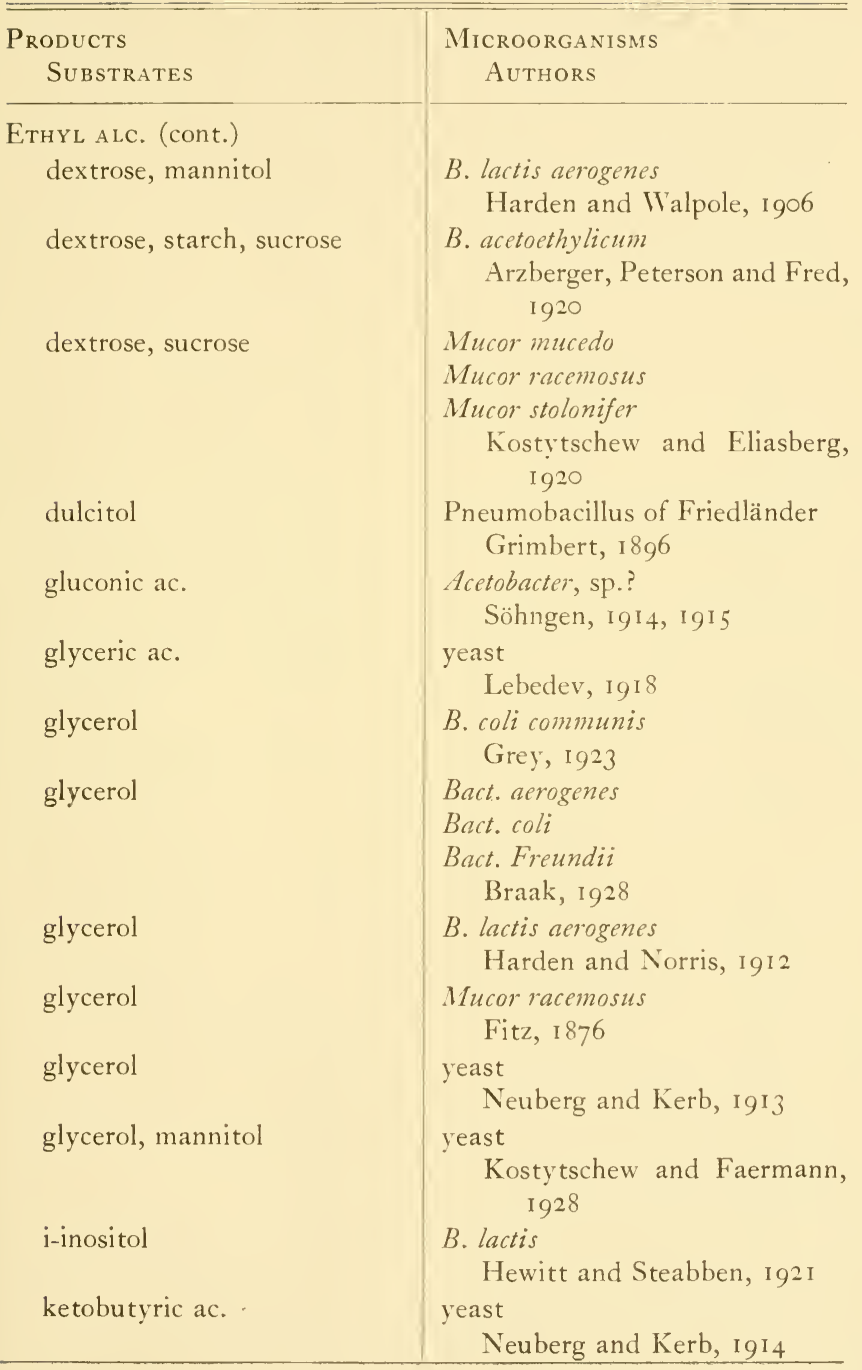




Products
Substrates

Ethyl Alc. (cont.)

lactic ac.

lactic ac.

lactose

lactose, maltose, mannitol, starch, sucrcse

malic ac.

malonic ac.

mannitol

mannitol

mannitol, glycerol

mannose

pyruvic ac.

pyruvic ac.

starch

starch

starch, sucrose

sucrose

sucrose
Microorganisms

Authors

B. ethacetosuccinicus

Mazé, I9I3

yeast

Kayser, 1923

B. coli

Grimbert, I 896

Amylobacter butylicus

Duclaux, 1895

B. coli communis

Grey, 1923

B. coli communis

Grey, I923

Pneumobacillus of Friedländer

Grimbert, I 896

B. ethaceticus

Frankland, Stanley and Frew, I 89 I

B. ethaceticus

Frankland and Fox, I889 yeast

Mezzadroli, I9 8 yeast

Neuberg and Kerb, I9I3 yeast

Lebedev and Polonski, I9I7

Granulobacterium butylicum

Folpmers, 192 I

Clostridium acetobutylicum

Killeffer, 1927

B. acetoethylicus

B. macerans

Bakonyi, 1926

Amylobacter ethylicus

Duclaux, 1895

B. invertenti-acetici

Mezzadroli, I9I7 


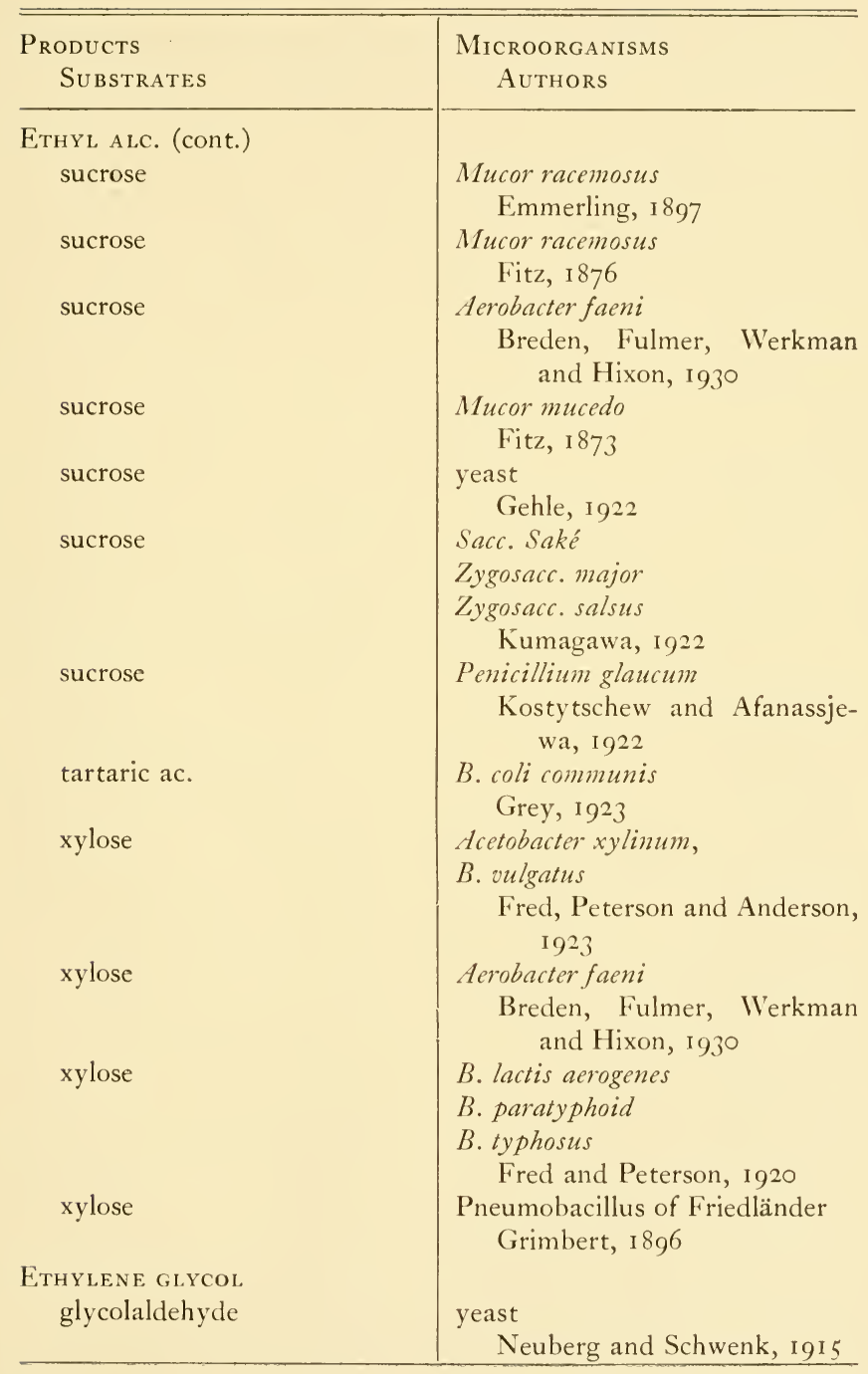




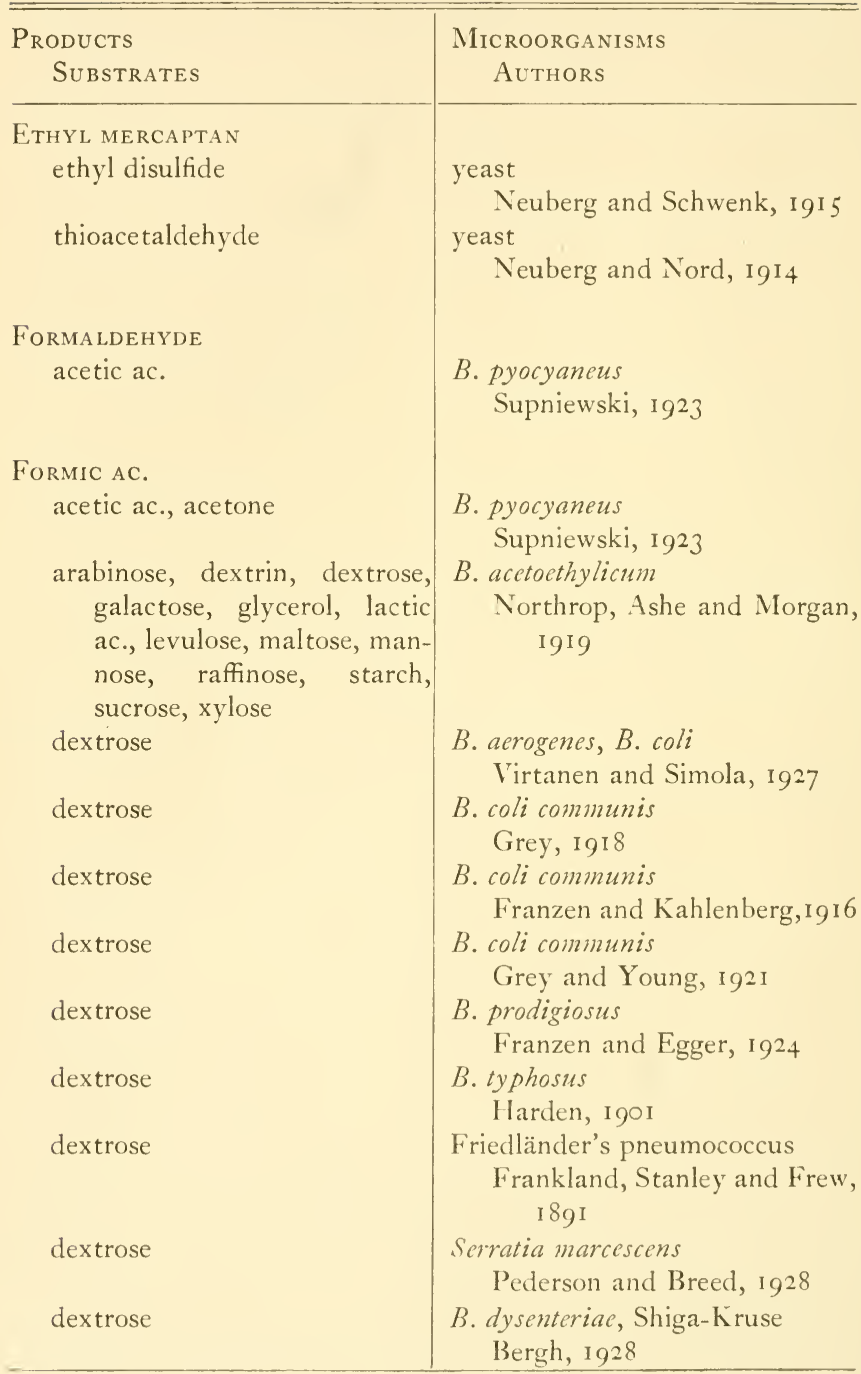




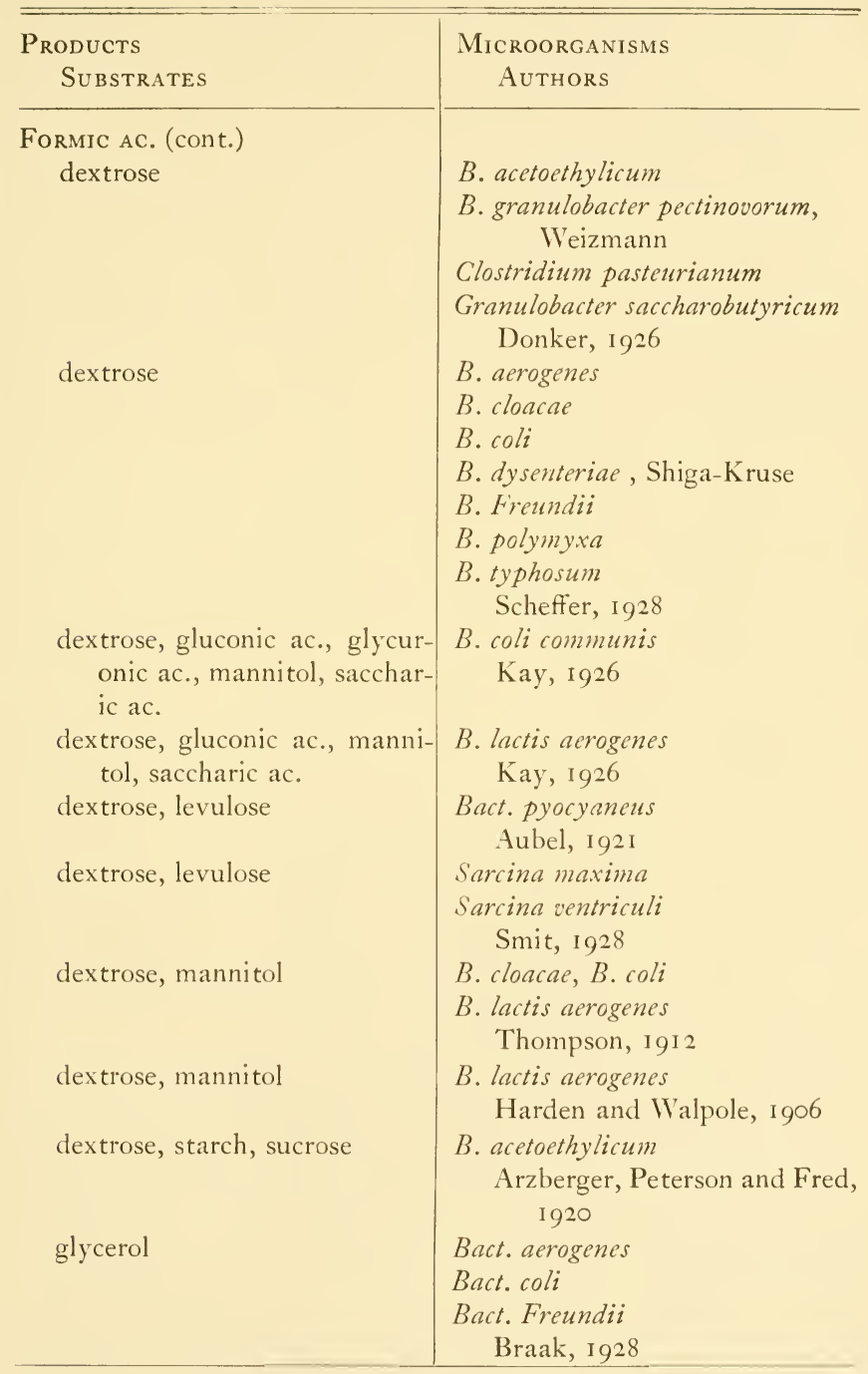




Products
Substrates

Formic ac. (cont.)

glycerol

glycerol

glycerol

lactic ac.

mannitol, glycerol

methyl alc.

pyruvic ac.

pyruvic ac.

quinic ac.

starch

starch

succinic ac.

sucrose

xylose

xylose
Microorganisms

Authors

B. butylicus

Buchner and Meisenheimer, 1908

$B$. lactis aerogenes

Harden and Norris, I9I 2

B. coli communis

Grey, I923

B. ethacetosuccinicus

Mazé, I913

B. ethaceticus

Frankland and Fox, 1889

B. pyocyaneus

Supniewski, 1923

B. coli, B. pyocyaneus

Cambier and Aubel, 1922

Bact. coli

Aubel, 1924

Schizomycetes

Loew, I 88 I

Pectinobacter amylophilum

Makrinov, 1915

$B$. suaveolens

Sclavo and Gosio, I89I

B. coli communis

Grey, I 923

Aerobacter faeni

Breden, Fulmer, Werkman and Hixon, 1930

B. paratyphoid

B. typhosus

Fred and Peterson, I920

Aerobacter faeni

Breden, Fulmer, Werkman and Hixon, 1930 
Products

Substrates

Fumaric ac.

arabinose, galactose

dextrose, levulose

malic ac., succinic ac.

pyruvic ac.

sucrose

Furfuryl alc.

furfural

Furyltrimethyleneglycol furfural

Galactonic ac. galactose

Geraniol citral

$\alpha$-Glucohe PTU Lose

a-glucoheptite

Gluconic ac.

arabinose, dextrose, glycerol, levulose, mannose, sucrose dextrose

dextrose
Microorganisms

Authors

Asp. fumaricus

Schreyer, 1928

Rhizopus nigricans

Ehrlich, I9I 2

$B$. coli communis

Quastel and Whetham, I924 Rhizopus nigricans

Gottschalk, I925

Asp.fumaricus

Wehmer, I918, 1928

yeast

Lintner and Liebig, I9I I

yeast

Lintner and Liebig, I9I 3

sorbose bacterium

Bertrand, 1898

yeast

Neuberg and Kerb, I918

Sorbose bacterium

Bertrand and Nitzberg, 1928

Asp. niger

Bernhauer, 1928

Asp. cinnamoneus

Asp.fuscus

Asp. niger

Falck and Kapur, 1924

Bact. aceti

Brown, I 886 


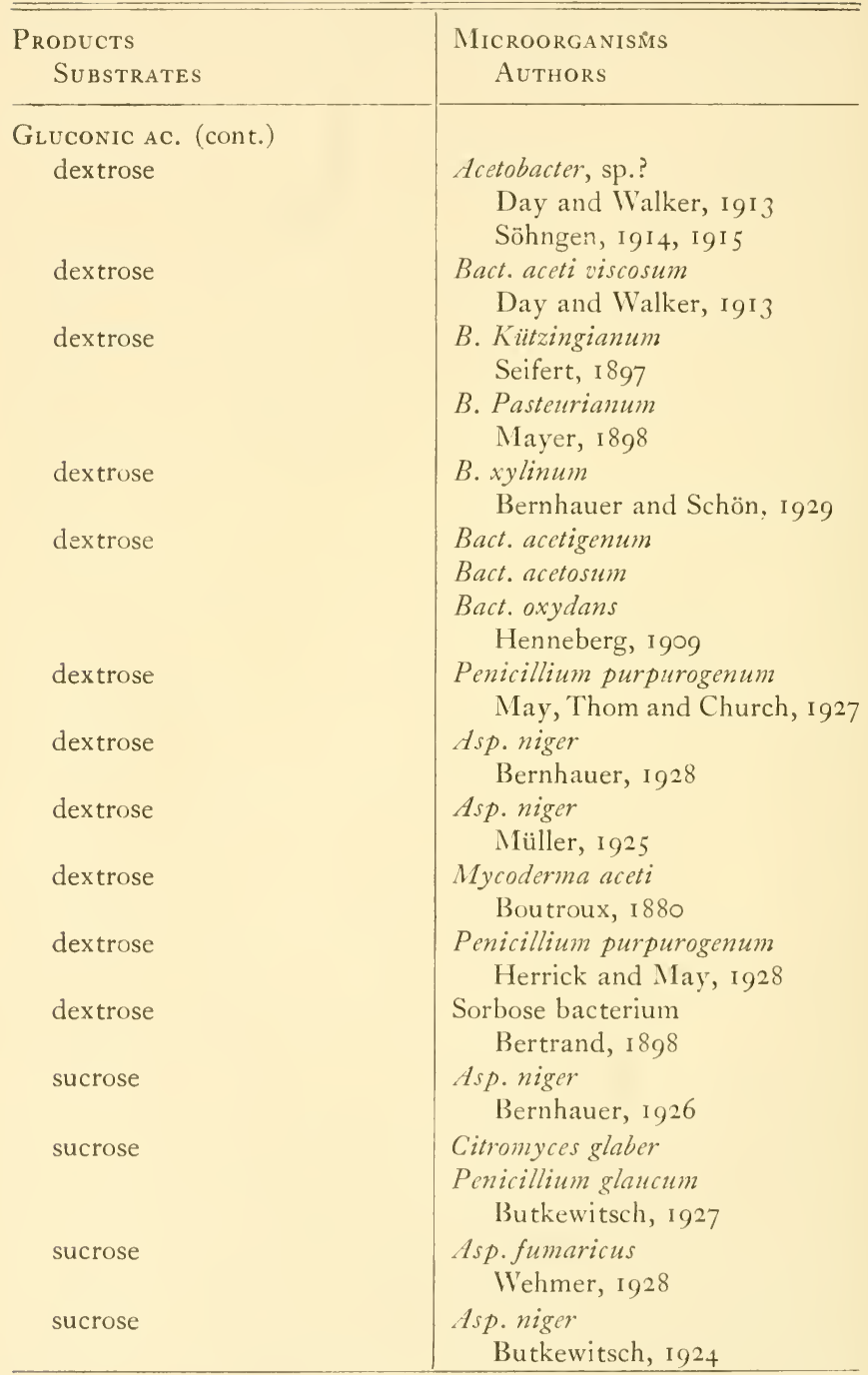




\begin{tabular}{|c|c|}
\hline $\begin{array}{l}\text { Products } \\
\text { Substrates }\end{array}$ & $\begin{array}{l}\text { Microorganisms } \\
\text { Authors }\end{array}$ \\
\hline \multicolumn{2}{|l|}{ Gluconic ac. (cont.) } \\
\hline sucrose & $\begin{array}{l}\text { Asp. niger } \\
\text { Bernhauer, I924, } 1926\end{array}$ \\
\hline $\begin{array}{l}\text { GLYCERIC AC. } \\
\text { glycerol }\end{array}$ & $\begin{array}{l}\text { B. pyocyaneus } \\
\text { Supniewski, I923 }\end{array}$ \\
\hline \multicolumn{2}{|l|}{ GLycerol } \\
\hline dextrose & $\begin{array}{l}\text { yeast } \\
\text { Oppenheimer, I9I3 }\end{array}$ \\
\hline dextrose, sucrose & $\begin{array}{l}\text { yeast } \\
\text { Neuberg and Hirsch, I9 I9 }\end{array}$ \\
\hline galactose & $\begin{array}{l}\text { yeast } \\
\text { Tomita, I92I }\end{array}$ \\
\hline sucrose & $\begin{array}{l}\text { Mucor racemosus } \\
\text { Emmerling, } 1897\end{array}$ \\
\hline sucrose & $\begin{array}{l}\text { Sacc. ellipsoideus } \\
\text { Adams, I9I9 }\end{array}$ \\
\hline sucrose & $\begin{array}{l}\text { Sacc. Saké, Zygosacc. major } \\
\text { Zygosacc. salsus } \\
\quad \text { Kumagawa, I922 }\end{array}$ \\
\hline sucrose & $\begin{array}{l}\text { Sterigmatocystis nigra } \\
\text { Molliard, I } 922\end{array}$ \\
\hline sucrose & $\begin{array}{l}\text { yeast } \\
\text { Zerner, I920 }\end{array}$ \\
\hline sucrose & $\begin{array}{l}\text { yeast } \\
\text { Abderhalden and Stix, I922 }\end{array}$ \\
\hline sucrose & $\begin{array}{l}\text { yeast } \\
\text { Gehle, 1922 }\end{array}$ \\
\hline $\begin{array}{l}\text { sucrose } \\
\text { sucrose }\end{array}$ & $\begin{array}{l}\text { yeast } \\
\text { Neuberg and Reinfürth, I923 } \\
\text { yeast }\end{array}$ \\
\hline & $\begin{array}{c}\text { Buchner and Meisenheimer, } \\
\text { I906 }\end{array}$ \\
\hline sucrose & $\begin{array}{l}\text { yeast } \\
\text { Tomoda, I928 }\end{array}$ \\
\hline
\end{tabular}




Products
Substrates

Glycogen dextrose

\section{Glycolaldehyde}

dihydroxymaleic ac.

hydroxy-pyruvic ac.

Glycollic AC. acetic ac., citric ac.

ethylene glycol

ethylene glycol

oxalic ac., pyruvic ac.

pyruvic ac.

n-HEPTYL ALC. enanthol

n-HEXYL ALC.

caproic aldehyde

$\beta$-Hydroxybutyric AC. acetaldol

butyric ac.

Isobutyl Alc. iso-butylaldehyde
Microorganisms

Authors

Sacc. Ludwigii

Gottschalk, I925

yeast

Neuberg and Schwenk, I9I 5 yeast

Neuberg and Kerb, I9I3

Asp. niger

Challenger, Subramaniam and Walker, 1927

B. Kützingianum

Seifert, I 897

B. Pasteurianum

Mayer, 1898

B. coli

$B$. proteus vulgatus

Cambier and Aubel, 1922 Bact. coli

Aubel, I924

yeast

Ohta, I9I4

yeast

Neuberg and Nord, 19I4

B. ascendens

Binder-Kotrba, I926

Asp. niger

Coppock, Walker and Subramaniam, 1928

yeast

Ohta, 1914 


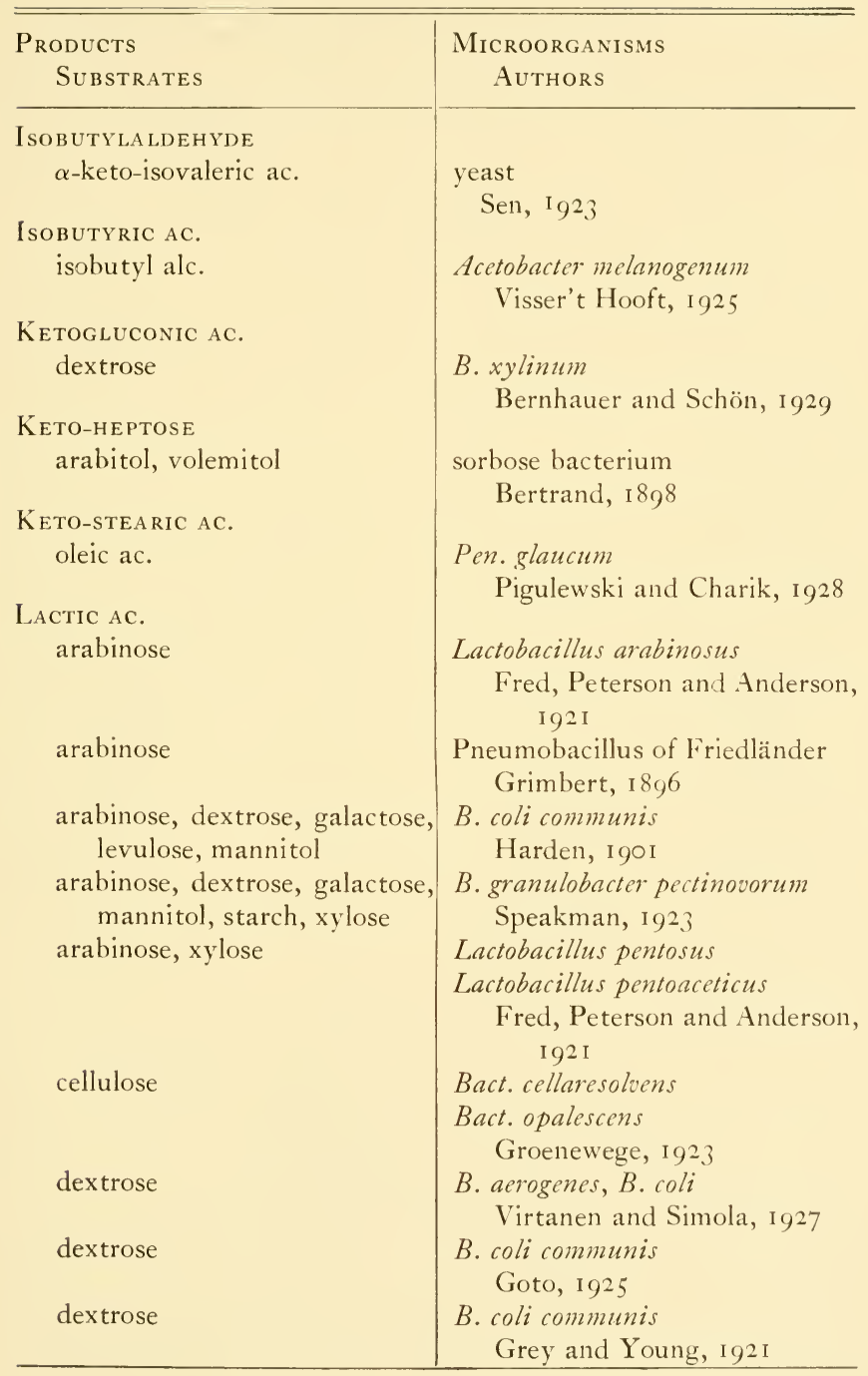




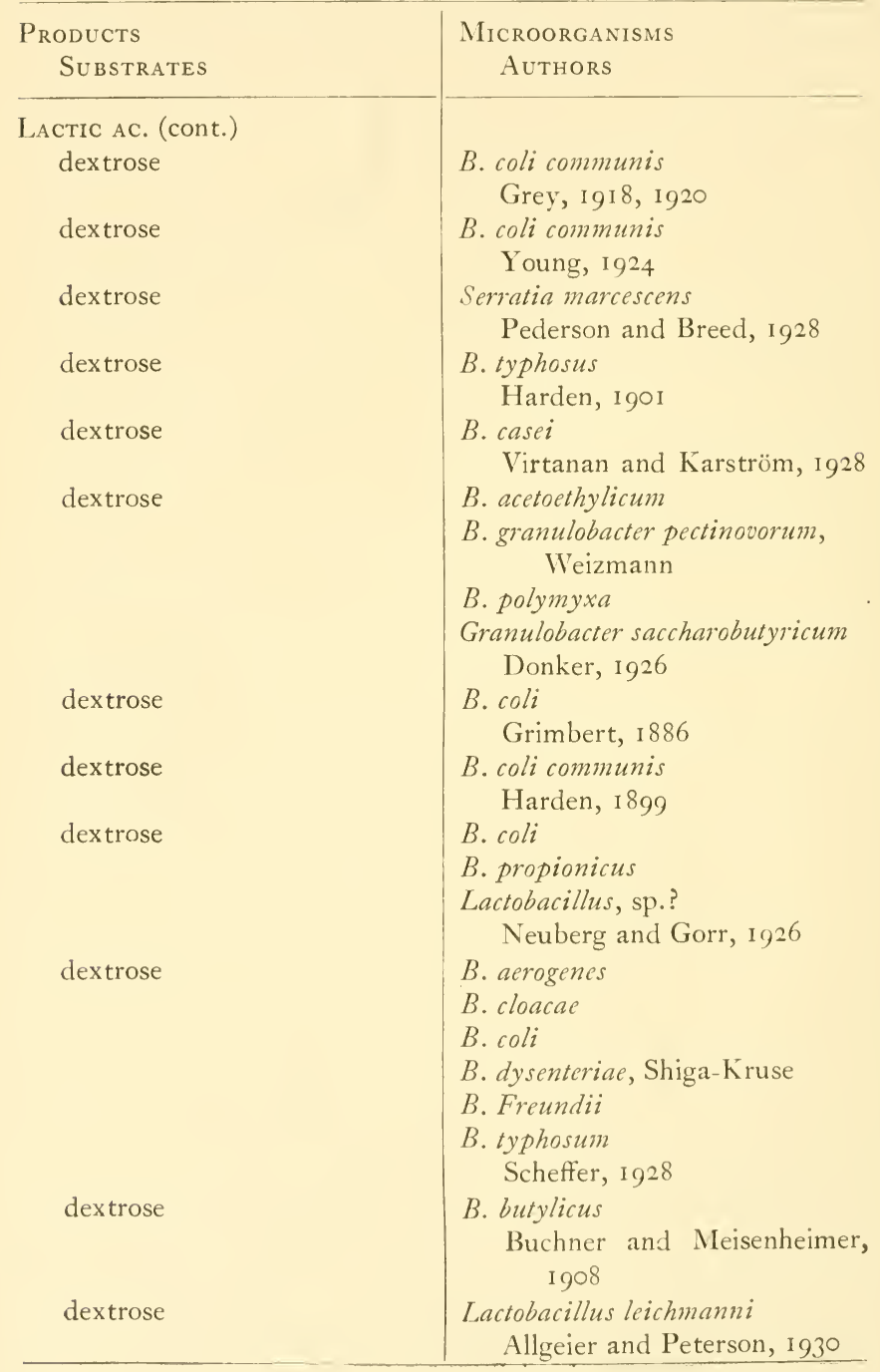




\begin{tabular}{|c|c|}
\hline $\begin{array}{l}\text { Products } \\
\text { Substrates }\end{array}$ & $\begin{array}{l}\text { Microorganisms } \\
\text { Authors }\end{array}$ \\
\hline \multicolumn{2}{|l|}{ Lactic ac. (cont.) } \\
\hline dextrose & $\begin{array}{l}\text { B. coli } \\
\text { Péré, I } 898\end{array}$ \\
\hline dextrose & $\begin{array}{l}\text { Rhizopus chinensis } \\
\text { Saito, I9I I }\end{array}$ \\
\hline dextrose & $\begin{array}{l}\text { B. of malignant oedema } \\
\text { King and Frankel, I } 890\end{array}$ \\
\hline dextrose & $\begin{array}{l}\text { Streptococcus lactis } \\
\quad \text { Algeier and Peterson, I } 930\end{array}$ \\
\hline dextrose & $\begin{array}{l}\text { yeast } \\
\quad \text { Aubel, I } 929\end{array}$ \\
\hline $\begin{array}{l}\text { dextrose, dextrin, maltose, su- } \\
\quad \text { crose }\end{array}$ & $\begin{array}{l}\text { B. Delbrïcki } \\
\text { B. lactis acidi } \\
\text { Henneberg, } 1903\end{array}$ \\
\hline $\begin{array}{l}\text { dextrose, dihydroxyacetone, } \\
\text { glyceraldehyde }\end{array}$ & $\begin{array}{l}\text { yeast } \\
\text { Oppenheimer, I9I } 3\end{array}$ \\
\hline dextrose, galactose, mannose & $\begin{array}{l}\text { Lactobacillus pentoaceticus } \\
\text { Peterson and Fred, I } 920\end{array}$ \\
\hline $\begin{array}{l}\text { dextrose, gluconic ac., glycur- } \\
\text { onic ac., mannitol, saccha- } \\
\text { ric ac. }\end{array}$ & $\begin{array}{l}\text { B. coli communis } \\
\text { Kay, I } 926\end{array}$ \\
\hline $\begin{array}{l}\text { dextrose, gluconic ac., manni- } \\
\quad \text { tol, saccharic ac. }\end{array}$ & $\begin{array}{l}\text { B. lactis aerogenes } \\
\text { Kay, } 1926\end{array}$ \\
\hline dex trose, glycerol & $\begin{array}{l}\text { B. subtilis } \\
\text { Vandevelde, I } 884\end{array}$ \\
\hline $\begin{array}{l}\text { dextrose, lactose, maltose, man- } \\
\text { nitol, sucrose }\end{array}$ & $\begin{array}{l}\text { B. tartricus } \\
\text { Grimbert, IgoI }\end{array}$ \\
\hline dextrose, levulose & $\begin{array}{l}\text { Clostridium thermocellum } \\
\text { Peterson, Fred and Marten, } \\
\text { I926 }\end{array}$ \\
\hline dextrose, levulose & $\begin{array}{l}\text { Sarcina maxima } \\
\text { Sarcina ventriculi } \\
\quad \text { Smit, } 1928\end{array}$ \\
\hline dextrose, mannitol & $\begin{array}{l}\text { B. coli communis } \\
\text { Grey, I9I } 8\end{array}$ \\
\hline
\end{tabular}




\begin{tabular}{|c|c|}
\hline $\begin{array}{l}\text { Products } \\
\text { Substrates }\end{array}$ & $\begin{array}{l}\text { Microorganisms } \\
\text { Authors }\end{array}$ \\
\hline \multirow{2}{*}{$\begin{array}{l}\text { Lactic ac. (cont.) } \\
\text { dextrose, mannitol }\end{array}$} & \\
\hline & $\begin{array}{l}\text { B. cloacae, } B . \text { coli } \\
\text { B. lactis aerogenes } \\
\text { Thompson, I91 } 2\end{array}$ \\
\hline dextrose, mannitol & $\begin{array}{l}\text { B. lactis aerogenes } \\
\text { Harden and Walpole, I } 906\end{array}$ \\
\hline dextrose, starch, sucrose & $\begin{array}{l}\text { B. acetoethylicum } \\
\text { Arzberger, Peterson and Fred, } \\
\text { I } 920\end{array}$ \\
\hline galactose & $\begin{array}{l}\text { B. coli } \\
\text { Péré, } 1898\end{array}$ \\
\hline glycerol & $\begin{array}{l}\text { Bact. aerogenes } \\
\text { B. coli } \\
\text { B. Freundii } \\
\quad \text { Braak, I } 928\end{array}$ \\
\hline glycerol & $\begin{array}{l}\text { B. lactis aerogenes } \\
\text { Harden and Norris, I } 9 \mathrm{I} 2\end{array}$ \\
\hline glycerol & $\begin{array}{l}\text { B. pyocyaneus } \\
\text { Supniewski, 1923 }\end{array}$ \\
\hline glycerol & $\begin{array}{l}\text { B. coli communis } \\
\text { Grey, } 1923\end{array}$ \\
\hline glycerol & $\begin{array}{l}\text { B. butylicus } \\
\text { Buchner and Meisenheimer, } \\
\text { I908 }\end{array}$ \\
\hline inositol & $\begin{array}{l}\text { B. lactis aerogenes } \\
\text { Kumagawa, } 1922\end{array}$ \\
\hline lactose & $\begin{array}{l}\text { Bact. caucasicum } \\
\text { Kostytschew and Soldaten- } \\
\text { kov, } 1927\end{array}$ \\
\hline lactose & $\begin{array}{l}\text { B. coli } \\
\text { Grimbert, I } 896\end{array}$ \\
\hline lactose & $\begin{array}{l}\text { B. coli } \\
\text { Péré, I } 898\end{array}$ \\
\hline lactose & $\begin{array}{l}\text { B. lactis acidi } \\
\text { Henneberg, } 1903\end{array}$ \\
\hline $\begin{array}{l}\text { lactose, maltose, mannitol, } \\
\text { starch, sucrose }\end{array}$ & $\begin{array}{l}\text { Amylobacter butylicus } \\
\text { Duclaux, I } 895\end{array}$ \\
\hline
\end{tabular}




\begin{tabular}{|c|c|}
\hline $\begin{array}{l}\text { Products } \\
\text { Substrates }\end{array}$ & $\begin{array}{l}\text { Microorganisms } \\
\text { Authors }\end{array}$ \\
\hline \multicolumn{2}{|l|}{ LACTIC AC. (cont.) } \\
\hline levulose & $\begin{array}{l}\text { Lactobacillus pentoaceticus } \\
\text { Peterson and Fred, } 1920\end{array}$ \\
\hline levulose & $\begin{array}{l}\text { Bact. pyocyaneus } \\
\text { Aubel, I92 I }\end{array}$ \\
\hline malic ac. & $\begin{array}{l}\text { Lactobacillus pentoaceticus } \\
\text { Peterson and Fred, I920 }\end{array}$ \\
\hline malic ac. & $\begin{array}{l}\text { yeast } \\
\text { Lebedev and Russ, 1916 }\end{array}$ \\
\hline malonic ac. & $\begin{array}{l}\text { B. coli communis } \\
\text { Grey, } 1925\end{array}$ \\
\hline mannitol & $\begin{array}{l}\text { B. coli } \\
\text { Péré, I } 898\end{array}$ \\
\hline mannitol & $\begin{array}{l}\text { Lactobacillus pentoaceticus } \\
\text { Peterson and Fred, I } 920\end{array}$ \\
\hline mannitol & $\begin{array}{l}\text { Pneumobacillus of Friedländer } \\
\text { Grimbert, } 1896\end{array}$ \\
\hline mannose & $\begin{array}{l}\text { B. coli } \\
\text { Péré, I } 898\end{array}$ \\
\hline methylglyoxal & $\begin{array}{l}\text { Bact. pasteurianum } \\
\text { Gorr and Perlman, I926 }\end{array}$ \\
\hline methylglyoxal & $\begin{array}{l}\text { Bact. coli } \\
\quad \text { Neuberg and Gorr, } 1925\end{array}$ \\
\hline methylglyoxal & $\begin{array}{l}\text { B. coli } \\
\text { B. propionicus } \\
\text { Lactobacillus, sp.? } \\
\quad \text { Neuberg and Gorr, } 1927\end{array}$ \\
\hline methylglyoxal & $\begin{array}{l}\text { B. Delbriucki } \\
\text { Bact. lactis aerogenes } \\
\text { Neuberg and Simon, } 1927\end{array}$ \\
\hline methylglyoxal & $\begin{array}{l}\text { Neuberg and Kobel, I927, } \\
\text { I929 }\end{array}$ \\
\hline methylglyoxal & $\begin{array}{l}\text { yeast } \\
\text { Neuberg, I9I3 }\end{array}$ \\
\hline methylglyoxal & $\begin{array}{l}\text { yeast } \\
\text { Dakin and Dudley, I9I } 3\end{array}$ \\
\hline
\end{tabular}




Products
Substrates
Lactic ac. (cont.)
d-propylene glycol
pyruvic ac.
pyruvic ac.
pyruvic ac.
pyruvic ac.

pyruvic ac.

starch

sucrose

sucrose

sucrose

sucrose

sucrose

sucrose

sucrose
Microorganisms

Authors

Bact. termo

LeBel, I 88 I

B. coli, B. proteus vulgatus

Cambier and Aubel, I922

Bact. coli

Aubel, 1924

B. pyocyaneus

Aubel, I924

Oidium albicans

O. farinosum

O. gueraldi

O. tenuis

Mazé and Ruot, I9I7

Rhizopus nigricans

Gottschalk, I925

Pectinobacter amylophilum

Makrinov, igi 5

Aerobacter faeni

Breden, Fulmer, Werkman and Hixon, $193^{\circ}$

Amylobacter ethylicus

Duclaux, 1895

B. acidi laevolactici

Schardinger, I 890

B. casei $E$

Virtanen, Wichmann and Lindström, I 927

B. coli

Virtanen and Simola, 1927

B. coli

Péré, I 898

B. invertenti-lattici

B. invertenti-acetici

Mezzadroli, I9I7 


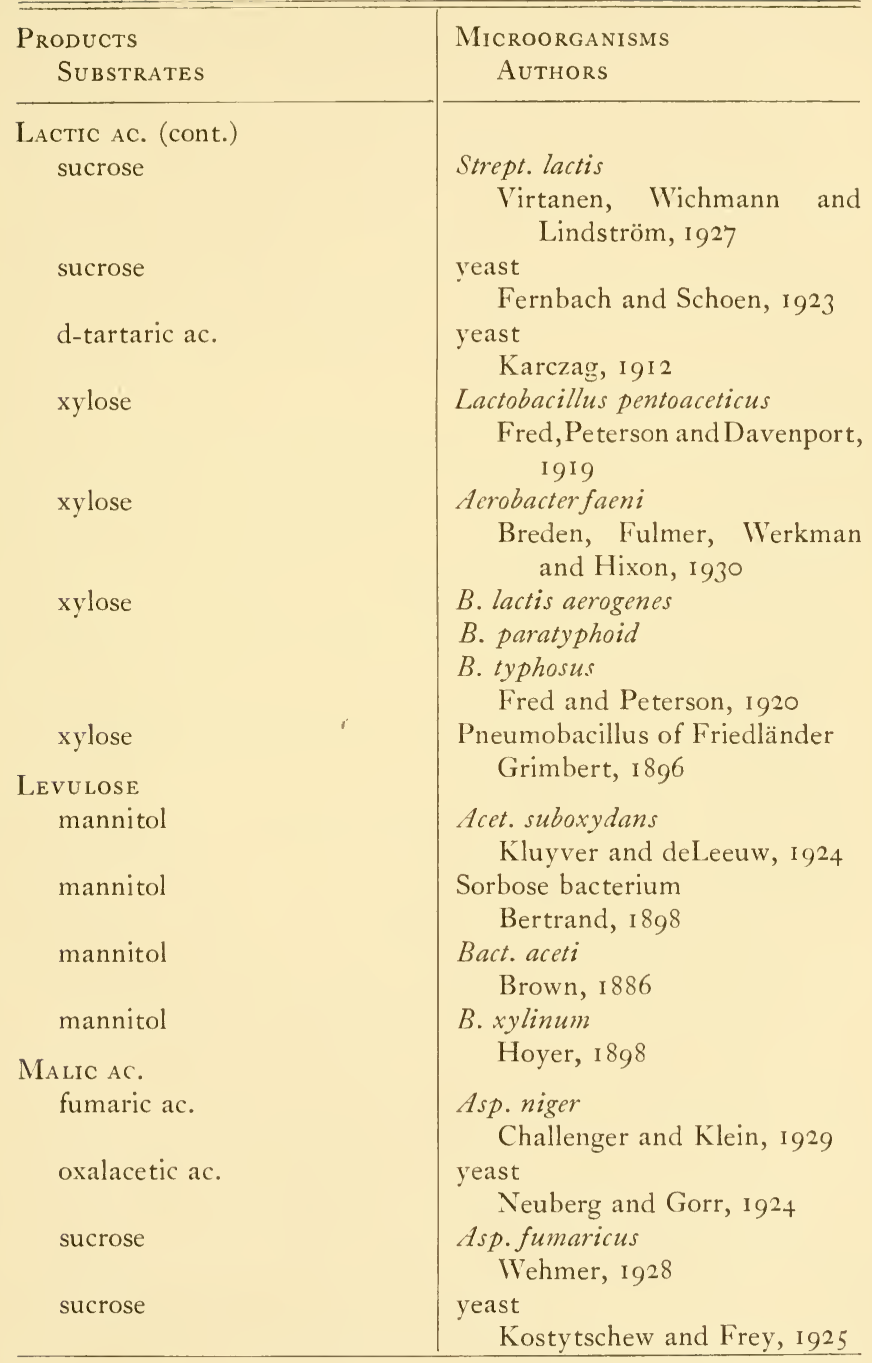




\begin{tabular}{|c|c|}
\hline $\begin{array}{l}\text { Products } \\
\text { Substrates }\end{array}$ & $\begin{array}{l}\text { Microorganisms } \\
\text { Authors }\end{array}$ \\
\hline $\begin{array}{l}\text { MaLIC AC. (cont.) } \\
\text { sucrose }\end{array}$ & $\begin{array}{l}\text { yeast } \\
\text { Dakin, } 19^{2} 4\end{array}$ \\
\hline $\begin{array}{l}\text { MANDELIC AC. } \\
\text { phenylglyoxal } \\
\text { phenylglyoxal }\end{array}$ & $\begin{array}{l}\text { yeast } \\
\text { Dakin and Dudley, I9I3 } \\
\text { Lactobacillus, sp.? } \\
\text { Mayer, I926 }\end{array}$ \\
\hline $\begin{array}{l}\text { Mannitol } \\
\text { dextrose } \\
\text { levulose }\end{array}$ & $\begin{array}{l}\text { B. subtilis } \\
\text { Vandevelde, } 188_{4} \\
\text { Lactobacillus pentoaceticus } \\
\text { Peterson and Fred, } 1920\end{array}$ \\
\hline $\begin{array}{l}\text { METHYLETHYLACETALDEHYDE } \\
\text { methylethyl pyruvic ac. }\end{array}$ & $\begin{array}{l}\text { yeast } \\
\text { Neuberg and Peterson, } 19{ }^{14}\end{array}$ \\
\hline $\begin{array}{l}\text { d-METHYLETHYLCARBINOL } \\
\text { methylethylketone + sucrose }\end{array}$ & $\begin{array}{l}\text { yeast } \\
\text { Neuberg and Nord, I919 }\end{array}$ \\
\hline $\begin{array}{l}\text { d-METHYLHEXYLCARBINOL } \\
\text { methyl-n-hexylketone+sucrose }\end{array}$ & $\begin{array}{l}\text { yeast } \\
\text { Neuberg and Nord, I9I9 }\end{array}$ \\
\hline $\begin{array}{l}\text { d-METHYLNONYLCARBINOL } \\
\text { methylnonylketone+ }+ \text { sucrose }\end{array}$ & $\begin{array}{l}\text { yeast } \\
\text { Neuberg and Nord, I9I9 }\end{array}$ \\
\hline $\begin{array}{l}\text { d-METHYL-n-PROPYLCARBINOL } \\
\text { methyl-n-propyl ketone+ } \\
\text { sucrose }\end{array}$ & $\begin{array}{l}\text { yeast } \\
\text { Neuberg and Nord, I919 }\end{array}$ \\
\hline $\begin{array}{l}\text { p-METHOXYBEnzoic AC. } \\
\text { p-methoxybenzaldehyde }\end{array}$ & $\begin{array}{l}\text { yeast } \\
\text { Neuberg and Liebermann, } \\
\text { I92 I }\end{array}$ \\
\hline $\begin{array}{l}\text { P-METHOXYBENZYL ALC. } \\
\text { p-methoxybenzaldehyde }\end{array}$ & $\begin{array}{l}\text { yeast } \\
\text { Neuberg and Liebermann, } \\
\text { I } 92 \mathrm{I}\end{array}$ \\
\hline
\end{tabular}




\section{Products}

Susbtrates

Methyl alc.

formaldehyde

Methylchlorethyl alc. methylchlorethyl ketone

O-METHYLCYCLOHEXANOL o-methylcyclohexanone

Methyl acetol

See acetylmethyl carbinol

Methylglyoxal lactose

sucrose

Methylphenylethylene glycoi methylbenzoyl carbinol

Oxalic ac. acetic ac.

acetic ac., arabinose, dextrose, ethylene glycol, galactose, glycerol, glycollic ac., lactic ac., malonic ac.

acetic ac., dextrose, erythritol, ethylene glycol, isolichenin, mannitol

acetic ac., dextrose, ery thritol, ethylene glycol, glycerol, levulose

acetic ac., dextrose, starch, tartaric ac.

\section{Microorganisms}

Authors

yeast

Neuberg and Welde, I9I4

yeast

Santomauro, 1924

yeast

Akamatsu, 1923

Bact. caucasicum

Kostytschew and Soldatenkov, 1927

B. coli

Virtanen and Simola, 1927

yeast

Neuberg and Komarewsky, 1927

Asp. niger

Challenger, Subramaniam and Walker, 1927

Termobacterium aceti

Banning, 1902

B. industrium

Banning, 1902

B. oxydans

Banning, 1902

Asp. niger

Wehmer, I 89 I 


\section{Products}

Substrates
Microorganisms

AUTHORS

Oxalic ac. (cont.)

acetic ac., fumaric ac., malic ac., succinic ac., tartaric ac. acetonedicarboxylic ac.

arabinose, dextrose, glycollic ac.

arabinose, dextrin, dextrose, ethylene glycol, galactose, glycollic ac., isobutyric ac. isolichenin, lactic ac., malonic ac.

arabinose, dextrin, dextrose, erythritol, ethylene glycol, glycollic ac., malonic ac.

arabinose, dextrose, ethyleneglycol, glycerol, glycollic ac., malonic ac., rhamnose arabinose, dextrose, glycerol, levulose, rhamnose

arabinose, dextrose, ery thritol, ethylene glycol, glycerol, glycollic ac., isobutyric ac., isolichenin, levulose, malonic ac., rhamnose

arabinose, dextrose, galactose, glycerose, levulose, mal tose, mannose, sucrose, xylose

arabinose, gluconic ac., glycerol, mannitol, saccharic ac., sucrose

citric ac.

citric ac.

Asp. niger

Raistrick and Clark, I9I9 Asp. niger

Walker, Subramaniam and Challenger, 1927

$B$. acetigenum

Banning, 1902

B. Kuitzingianum

Banning, I 902

B. acetosum

Banning, 1902

B. ascendens

Banning, 1902

B. xylinum

Banning, 1902

B. aceti

Banning, I 902

Asp. niger

Amelung, 1927

Asp. niger, Citromyces glaber

Amelung, 1927

Citromyces glaber

Butkewitsch, 1922

Asp. niger

Challenger, Subramaniam and Walker, I927 


\begin{tabular}{|c|c|}
\hline $\begin{array}{l}\text { Products } \\
\text { Substrates }\end{array}$ & $\begin{array}{l}\text { Microorganisms } \\
\text { Authors }\end{array}$ \\
\hline Oxalic ac. (cont.) & \\
\hline $\begin{array}{l}\text { dextrin, dextrose, ethylene gly- } \\
\text { col, glycerol, glycollic ac., } \\
\text { isobutyric ac., malonic ac., } \\
\text { sucrose }\end{array}$ & $\begin{array}{l}\text { B. Pasteurianum } \\
\text { Banning, } 1902\end{array}$ \\
\hline $\begin{array}{l}\text { dextrin, dextrose, glycerol, } \\
\text { inulin, lactose, mannitol, } \\
\text { sucrose }\end{array}$ & $\begin{array}{l}\text { Asp. niger } \\
\text { EIf ving, I9I9 }\end{array}$ \\
\hline dextrose & $\begin{array}{l}\text { Asp. niger } \\
\text { IVehmer, 1897, I } 924\end{array}$ \\
\hline dextrose & $\begin{array}{l}\text { Asp. niger } \\
\text { Heinze, } 1903\end{array}$ \\
\hline dextrose & $\begin{array}{l}\text { Citromyces glaber } \\
\text { Butkewitsch, } 1922\end{array}$ \\
\hline ethyl alc. & $\begin{array}{l}\text { Citromyces citricus } \\
\text { Citromyces tartricus } \\
\quad \text { Mazé and Perrier, } \mathrm{IgO}_{4}\end{array}$ \\
\hline raffinose & $\begin{array}{l}\text { Asp. niger } \\
\quad \text { Gillot, I } 899\end{array}$ \\
\hline sucrose & $\begin{array}{l}\text { Asp. niger } \\
\text { Bernhauer, } 1926\end{array}$ \\
\hline sucrose & $\begin{array}{l}\text { Asp. niger } \\
\text { Currie, I9I7 }\end{array}$ \\
\hline sucrose & $\begin{array}{l}\text { Asp. niger } \\
\quad \text { Butkewitsch, 1923, 1924 }\end{array}$ \\
\hline sucrose & $\begin{array}{l}\text { Asp. niger } \\
\quad \text { Bernhauer, } 19^{2}+\end{array}$ \\
\hline sucrose & $\begin{array}{l}\text { Citromyces citricus } \\
\text { Citromyces glaber, } \\
\text { Citromyces Pfefferianus, } \\
\text { Penicillium glaucum } \\
\quad \text { Butkewitsch, } 1922\end{array}$ \\
\hline sucrose & $\begin{array}{l}\text { Sterigmatocystis nigra } \\
\text { Molliard, } 1922\end{array}$ \\
\hline $\begin{array}{l}\beta \text {-oxyButyric Ac. } \\
\text { acetaldol }\end{array}$ & $\begin{array}{l}\text { Bact. ascendens } \\
\text { Binder-Kotrba, } 1926\end{array}$ \\
\hline
\end{tabular}


I56 CHEMICAL ACTION OF MICROORGANISMS

Products

Substrates

OxYgLUCONIC AC.

gluconic ac.

dextrose

Oxymaltol

glycerol

Perseulose

perseitol

d-perseitol

1-PHENYLACETYLCARBINOL benzaldehyde

Phenylethyl alc.

phenylacetaldehyde

1-PHENYLMETHYLCARBINOL ace tophenone + sucrose

Phloroglucinol phloridzin

Phenylpropylaldehyde benzylpyruvic ac.

Propionaldehyde $a$-ketobutyric ac.

Propionic ac.

arabinose, xylose

dextrose
Microorganisms

Authors

Acetobacter suboxydans

Kluyver and deLeeuw, 1924 Micrococcus oblongus

Boutroux, I 886

Asp. glaucus

Traetta-Mosca and Preti, I92 I

Sorbose bacterium

Bertrand, 1898

Bact. xylinum

Bertrand, I 909

yeast

Neuberg and Ohle, 1923

yeast

Neuberg and Welde, I914

yeast

Neuberg and Nord, I9I9

Asp. niger, Cladosporium, sp.?

Boas, I9I 6

yeast

Rona, I9'4

yeast

Neuberg and Kerb, 1912, 1913

Propionibacterium pentosaceum

Werkman, Hixon, Fulmer and Rayburn, 1929

Diplococcus pneumoniae

Brieger, I 883 


Products
Substrates
Propionic ac. (cont.)
dextrose, glycerol, lactic ac.

Microorganisms

Authors

dextrose, lactic ac., lactose, pyruvic ac. lactic ac.

lactic ac.

lactic ac.

lactose

propyl alc.

propyl alc.

propyl alc.

d-propyleneglycol

pyruvic ac.

Propionibacterium Freudenreichii

Propionibacterium Fensenii

Propionibacterium pentosaceum

Propionibacterium Peterssonii

Propionibacterium rubrum

Propionibacterium Shermanii

Propionibacterium technicum

Propionibacterium Thonii

van Niel, 1928

Bact. acidi propionici

Virtanen, 1923

B. acidi propionici

$B$. acidi propionici-a

$B$. acidi propionici-b

von Freudenreich and Jensen, I 906

B. subtilis

Fitz, I 880

B. casei

B. mobilis

Micrococcus casei liquefaciens

Orla-Jensen, 1904

Bact. acidi propionici

Sherman and Shaw, 1923

Bact. aceti

Brown, i 886

Acetobacter, sp.?

$B$. aceti (Hansen)

Buchner and Gaunt, I9o6

B. Kützingianum

B. Pasteurianum

Mayer, 1898

Bact. termo

LeBel, I88 I

Bact. coli

Aubel, I924 


\begin{tabular}{|c|c|}
\hline $\begin{array}{l}\text { Products } \\
\text { Substates }\end{array}$ & $\begin{array}{l}\text { Microorganisms } \\
\text { Authors }\end{array}$ \\
\hline \multicolumn{2}{|l|}{ Propionic ac. (cont.) } \\
\hline quinic ac. & $\begin{array}{r}\text { Schizomycetes } \\
\text { Loew, I } 88 \text { I }\end{array}$ \\
\hline succinic ac. & $\begin{array}{l}\text { B. pyocyaneus } \\
\text { Quastel, I } 924\end{array}$ \\
\hline succinic ac. & $\begin{array}{l}B \text { casei } \\
\quad \text { Orla-Jensen, } \mathrm{I}_{904}\end{array}$ \\
\hline tartaric ac. & $\begin{array}{l}\text { Bact. termo } \\
\text { König, I } 88 \text { I }\end{array}$ \\
\hline d-tartaric ac. & $\begin{array}{l}\text { yeast } \\
\text { Karczag, I9I2 }\end{array}$ \\
\hline \multicolumn{2}{|l|}{ Propyl alc. } \\
\hline glycerol & $\begin{array}{l}\text { B. butylicus } \\
\text { Morin, I } 887\end{array}$ \\
\hline a-ketobutyric ac. & $\begin{array}{l}\text { yeast } \\
\quad \text { Neuberg and Kerb, I9I4 }\end{array}$ \\
\hline starch & $\begin{array}{l}\text { Granulobacterium butylicum } \\
\text { Folpmers, I } 921\end{array}$ \\
\hline \multicolumn{2}{|l|}{$\begin{array}{l}\text { Protocatechuic ac. } \\
\text { quinic ac. }\end{array}$} \\
\hline & $\begin{array}{l}\text { Micrococcus chinicus } \\
\text { Emmerling and Abderhalden, } \\
\text { I903 }\end{array}$ \\
\hline quinic ac. & $\begin{array}{l}\text { Schizomycetes } \\
\text { Loew, I } 881\end{array}$ \\
\hline quinic ac. & $\begin{array}{l}\text { Asp. niger, Asp. oryzae } \\
\text { Citromyces glaber } \\
\quad \text { Butkewitsch, } 192+\end{array}$ \\
\hline $\begin{array}{l}\text { Pyrocatechol } \\
\text { quinic ac. }\end{array}$ & $\begin{array}{l}\text { Asp. niger, Asp. oryzae } \\
\text { Citromyces glaber } \\
\quad \text { Butkewitsch, } 1924\end{array}$ \\
\hline \multicolumn{2}{|l|}{$\begin{array}{l}\text { Pyroracemic ac. } \\
\text { See pyruvic ac. }\end{array}$} \\
\hline $\begin{array}{l}\text { Pyruvic ac. } \\
\text { dextrose }\end{array}$ & $\begin{array}{l}\text { B. coli } \\
\text { Aubel, I926 }\end{array}$ \\
\hline
\end{tabular}




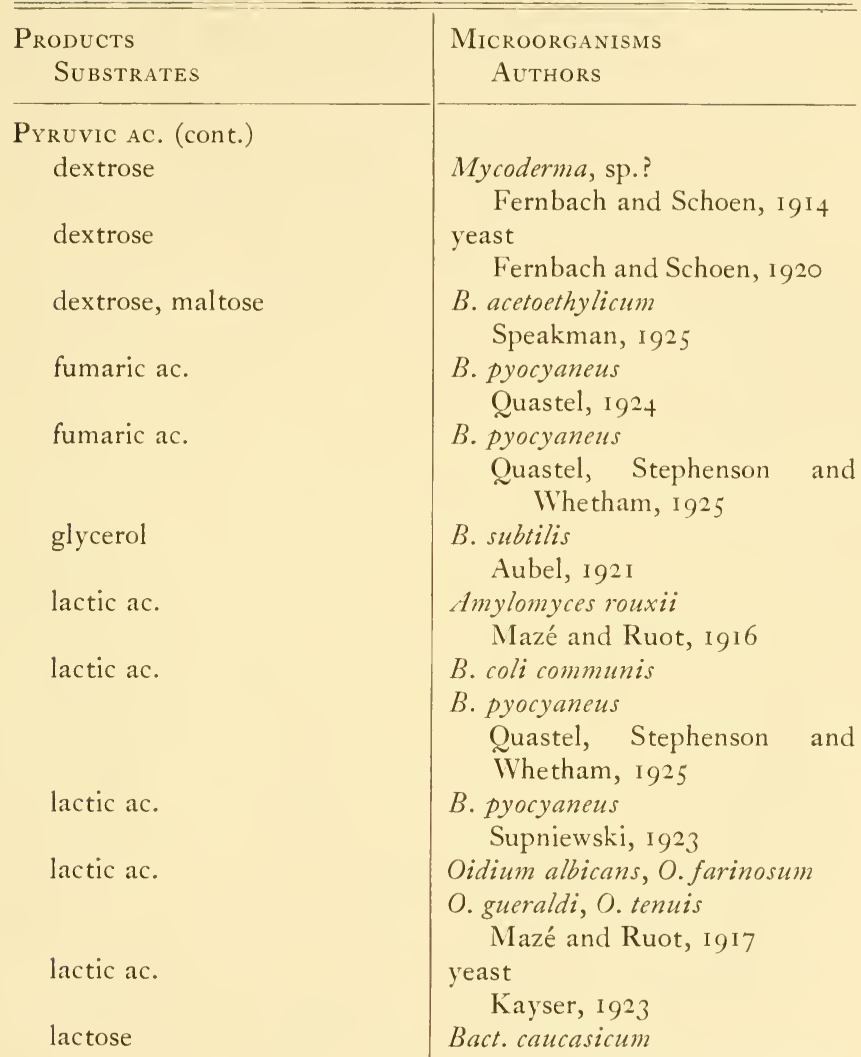

Kosty tschew andSoldatenkov, I 927

malic ac.

sucrose

sucrose

B. fluorescens

Beijerinck, I 9 I 6

yeast

Fernbach and Schoen, I9I3 yeast

Kostytschew and Soldatenkov, I 928 


\begin{tabular}{l} 
Products \\
Substrates \\
\hline
\end{tabular}

SACCHARIC AC.

gluconic ac.

Sorbose

sorbitol

sorbitol

d-sorbitol

StYrol

cinnamic ac.

Succinic ac.

acetaldehyde

aldehydropropionic ac.

arabinose

arabinose, dextrose, galactose, levulose, mannitol citric ac.

citric ac.

dextrose

dextrose
Microorganisms

Authors

Asp. niger

Walker, Subramaniam and Challenger, 1927

Acetobacter suboxydans

Kluyver and deLeeuw, 1924

B. xylinum

Seifert, I 897 , Mayer, 1898

Sorbose bacterium

Bertrand, 1898

Asp. niger

Herzog and Ripke, I908

$B$. lactis aerogenes

Harden and Norris, I91 2 yeast

Neuberg and Ringer, I918

B. ethaceticum

Frankland and MacGregor, 1892

B. coli communis

Harden, I90 I

B. coli communis

Grey, 1923

B. suipestifer

Brown, Duncan and Henry, 1924

B. dysenteriae, Shiga-Kruse

Bergh, I 928

$B$. aerogenes

B. coli

B. dysenteriae, Shiga-Kruse

B. Freundii

B. typhosum

Scheffer, I 928 
Products

Substrates

Succinic ac. (cont.)

dextrose

dextrose

dextrose

dextrose

dextrose

dextrose

dextrose

dextrose, gluconic ac., glycuronic ac., mannitol, saccharic ac.

dextrose, gluconic ac., mannitol, saccharic ac. dextrose, glycerol, lactic ac.
Microorganisms

Authors

B. aerogenes, B. coli

Virtanen and Simola, 1927

$B$. coli communis

Grey and Young, I92I

$B$. coli communis

Grey, I9I 8

$B$. coli communis

Young, 1924

B. typhosus

Harden, I 90 I

Oidium lupuli

Sumiki, 1927

Serratia marcescens

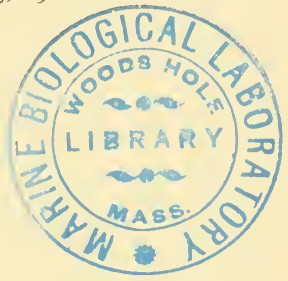

Pederson and Breed, 1928

B. coli communis

Kay, 1926

B. lactis aerogenes

Kay, 1926

Propionibacterium Freudenreichii

Propionibacterium pentosaceum

Propionibacterium Peterssonii

Propionibacterium rubrum

Propionibacterium Shermanii

Propionibacterium Thonii

van Niel, 1928

dextrose, glycerol, lactic ac.,

pyruvic ac., starch

dextrose, glycerol, lactic ac.

dextrose, lactose

dextrose, lactose, maltose, mannitol, sucrose

Propionibacterium technicum

van Niel, I 928

Propionibacterium Fensenii

van Niel, 1928

Bact. acidi propionici, var.fuscum

Virtanen, I923

B. tartricus

Grimbert, I90 I 


\section{Products}

Substrates

Succinic ac. (cont.)

dextrose, mannitol

dextrose, mannitol

dextrose, mannitol

dulcitol

dulcitol, mannitol

fumaric ac.

glycerol

glycerol

glycerol

glycerol

glycerol

inositol

i-inositol

a-ketoglutaric ac.

ketoglutaric ac.
Microorganisms

Authors

B. cloacae, B. coli

$B$. lactis aerogenes

Thompson, I9I 2

$B$. lactis aerogenes

Harden and Walpole, 1906

B. coli communis

Grey, I9I 8

Pneumobacilus of Friedländer

Grimbert, 1896

B. ethacetosuccinicus

Frankland and Frew, I 892

B. pyocyaneus

Quastel, Stephenson and Whetham, 1925

Bact. aerogenes

Bact. coli

Bact. Freundii

Braak, 1928

$B$. lactis aerogenes

Harden and Norris, 1912

Rhizopus, sp?

Takahashi and Sakaguchi, I 927

B. subtilis

Vandevelde, I884

B. coli communis

Grey, 1923

$B$. lactis aerogenes

Kumagawa, 1922

B. lactis

Hewitt and Steabben, 1921

B. xylinum

Iwatsuru, 1925

yeast

Neuberg and Ringer, I9I4 


\begin{tabular}{|c|c|}
\hline $\begin{array}{l}\text { Products } \\
\text { Substrates }\end{array}$ & $\begin{array}{l}\text { Microorganisms } \\
\text { Authors }\end{array}$ \\
\hline \multicolumn{2}{|l|}{ Succinic ac. (cont.) } \\
\hline lactose & $\begin{array}{l}\text { B. lactis aerogenes } \\
\text { Emmerling, I } 900\end{array}$ \\
\hline lactose & $\begin{array}{l}\text { B. coli } \\
\text { Grimbert, } 1896\end{array}$ \\
\hline levulose & $\begin{array}{l}\text { Sarcina maxima } \\
\text { Smit, } 1928\end{array}$ \\
\hline malic ac. & $\begin{array}{l}\text { B. coli communis } \\
\text { Grey, } 1923\end{array}$ \\
\hline malic ac. & $\begin{array}{l}\text { B. lactis aerogenes } \\
\text { Emmerling, } 1899\end{array}$ \\
\hline mannitol, glycerol & $\begin{array}{l}\text { B. ethaceticus } \\
\text { Frankland and Fox, I } 889\end{array}$ \\
\hline starch & $\begin{array}{l}\text { B. subtilis } \\
\text { Fitz, I } 878\end{array}$ \\
\hline starch & $\begin{array}{l}\text { Pectinobacter amylophilum } \\
\text { Makrinov, I9I } 5\end{array}$ \\
\hline sucrose & $\begin{array}{l}\text { Aerobacter faeni } \\
\text { Breden, Fulmer, Werkman } \\
\text { and Hixon, I } 930\end{array}$ \\
\hline sucrose & $\begin{array}{l}\text { Mucor mucedo } \\
\text { Fitz, I } 873\end{array}$ \\
\hline sucrose & $\begin{array}{l}\text { Mucor racemosus } \\
\text { Emmerling, I } 897\end{array}$ \\
\hline sucrose & $\begin{array}{l}\text { yeast } \\
\text { Kostytschew and Frey, I925 }\end{array}$ \\
\hline tartaric ac. & $\begin{array}{l}\text { B. coli communis } \\
\text { Grey, } 1923\end{array}$ \\
\hline tartaric ac. & $\begin{array}{l}\text { B. tartaricus } \\
\text { Grimbert and Ficquet, } 1898\end{array}$ \\
\hline tartaric ac. & $\begin{array}{l}\text { Bact. terno } \\
\text { König, I } 88 \text { I }\end{array}$ \\
\hline d-tartaric ac. & $\begin{array}{l}\text { yeast } \\
\quad \text { Karczag, I9I } 2\end{array}$ \\
\hline xylose & $\begin{array}{l}\text { Aerobacter faeni } \\
\text { Breden, Fulmer, Werkman } \\
\text { and Hixon, I } 930\end{array}$ \\
\hline
\end{tabular}




\section{Products}

Substrates

Succinic ac. (cont.)

xylose

xylose

O-TOLYLACETYL CARBINOL

o-tolylaldehyde

P-TOLYLACETYL CARBINOL

p-tolylaldehyde

2, 2, 3-TRICH LORBUTANOL

2, 2, 3-trichlorbutylaldehyde

TRICHLORETHYLALCOHOL trichloracetaldehyde

Trimethylene glycol glycerol

n-VALERALDEHYDE

$a$-keto-n-caproic ac.

VALERIC AC.

lactic ac.

methyl-ethyl-acetaldehyde

methyl-ethyl pyruvic ac.

Xylonic ac.

xylose

Arlose. (?)

xylan
Microorganisms

Authors

Pneumobacillus of Friedländer

Grimbert, I 896

B. paratyphoid, B. typhosus

Fred and Peterson, I920

yeast

Behrens and Iwanoff, 1926

yeast

Behrens and Iwanoff, I926

yeast

Rosenfeld, 1925

yeast

Lintner and Lüers, I9I3

Bact. Freundii

Braak, I928

yeast

Sen, 1923

B. subtilis

Fitz, I 880

B. ascendens

B. pasteurianum

B. xylinum

Neuberg and Simon, 1926 yeast

Neuberg and Peterson, I9I 4

Sorbose bacterium

Bertrand, I9I3

Actinomyces sp.?

Patrick, Werkman and Hixon, I 9.30 
CHAPTER IV

REFERENCES 



\section{CHAPTER IV}

\section{REFERENCES}

Аввотт, O. D., The utilization of pentoses by yeasts, and the composition of plant gums. Univ. of Mo. Agr. Expt. Sta. Res. Bul. No. 85: 1-29 (I926).

Abderhalden, Emil and Stix, Walter, Untersuchungen über die alkoholische Gärungmittels. Hefezellen unter verschiedenen Bedingungen. VIII. Mitteilung. Bildung von Glycerin beim Abfangen der Zwischenstufe Azetaldehyd durch Tierkohle. Fermentforschung 6: $345^{-7}$ (1922).

Acklin, Oskar and Schneider, Walter, Zur Biochemie des Penicillium glaucum. Ein Beitrag zum Problem der Methylketonbildung aus Triglyceriden bzw. Fettsäuren in Stoffwechsel des Schimmelpilzes-Biochem. Z. 202: 246-83 (1928)

Adams, A. B., Production of glycerine from sugar by fermentation. Chem. Trade J. 64: 385-6 (1919).

ÅARD, T., Enzymic hydrolysis of melezitose and turanose. Tids. Kemi Bergvesen 8: 5-9, 16-20, 35-9 (1928). After Chem. Absts. Akamatse, S., Phytochemische Reduktion in der Cyclohexanreihe.

Biochem. Z. 142: i 88-90 (1923).

Allen, A. H., and Moor, C. G., Vinegar. The Analyst 18: i80-3 (I893).

Allgeier, R. J., and Peterson, W. H., Bacterial production of the isomers of lactic acid and their identification. J. Bact. 19: I8 (1930).

Amelung, H., Beiträge zur Säurebildung durch Aspergillus niger. Z. Physiol. Chem. 166: 16I-209 (I927).

Anderson, Arthur K., and Willaman, J. J., The fermentation of glucose by Fusarium lini. Proc. Exp. Biol. Med. 20: 108-10 (1922).

Arzberger, C. F., Peterson, IV. H., and Fred, E. B., Certain factors that influence acetone production by Bacillus acetocthylicum. J. Biol. Chem. 44: +65-79 (1920).

Aubel, E., Action du bacille pyocyanique sur l'asparagine. Compt. rend. 173: 179-80 (1921).

Auber, E., Ataque du glucose et du levulose par le bacille pyocanique. Compt. rend. 173: I $493^{-5}$ (I92 I). 
Auber, E., Oxydation de la glycerine par le Bacillus subtilis. Compt. rend. soc. biol. 84: 574-6 (i 92 i).

Auber, E., Remarques sur la croissance du Bacille coli en milieu chimiquement dèfini. Ann. physiol. physicochim. biol. 2: 7394 (1926).

Auber, E., Au sujet du rapport ontre la production d'acide lactique et la croissance de la levure. Compt. rend. 188: 578-80 (1929). Austrian Patent i io,536. Working up stable manure, rancid fodder and other fermentable substances. Apr. 15, 1928. After Chemical Absts.

Bakonyi, Stefan, Versuche zur Theorie der Aceton-Aethylalkoholischen Gärung. Biochem. Z. 169: 125-8 (1926).

Banning, F., Zur Kenntnis der Oxalsäurebildung durch Bakterien. Centr. Bakt. Parasitenk., II Abt., 8: 395-8; 425-4I, 453-6, 520-5, 556-67 (1902).

Bayo, César Pi-Suñer, Gewinnung von Methylglyoxal bei der Spaltung von Zucker durch Hefen-mazerationssaft. Biochem. Z. 213: 489-94 (1929).

Behrens, Martin and Ifanoff, Nikolai N., Neue Versuche über die Carboligase. Biochem. Z. 169: 478-8I (I 926).

Beijerinck, M. W., Ueber die Arten der Essigbakterien. Centr. Bakt. Parasitenk., II Abt., 4: 209 (1898).

Beijerinck, M. W., Ueber Chinonbildung durch Streptothrix chromogena und Lebensweise dieses Mikroben. Centr. Bakt. Parasitenk. II. Abt. 6: 2-12 (1900).

Beijerinck, M. W., and Folpmers, T., Vorming von brandigdruivenzuur uit appelzuur door bakterien. Proc. K. Akad. Wetensch; Amsterdam, 18: 1198-1200 (1916).

Bernhauer, K., Zum Problem der Säurebildung durch Aspergillus niger. Biochem. Z. 153: 517-21 (1924).

Bernhauer, K., Über die Säurebildung durch Aspergillus niger. II. Die Bildung der Gluconsäure. Biochem. Z. 172: $3^{1} 3^{-2} 3$ (1926).

Bernhauer, K., Über die Säurebildung durch Aspergillus niger. III. Die Bildung der Citronensäure. Biochem. Z. 172: 32449 (1926).

Bernhauer, K., Zur Kenntniss der Glucoxydase Bildung. Z. Physiol. Chem. 177: 86-106 (I928).

Bernhauer, K., Über die Säurebildung durch Aspergillus niger. IV. Die Bedeutung der Mycelentwicklung für die Säurebildung. Biochem. Z. 197: 287-308 (1928). 
Bernhauer, K., Über die Charakterisierung der Stämme von Aspergillus niger auf Grund ihres biochemischen Verhaltens. Biochem.Z. 197: 278-86 (1928).

Bernhauer, K., and Schön, K., Zum Chemismus der Citronen Säurebildung durch Pilze. III. Über die Hypothesen und das Auftreten von Acetaldehyd in den Pilz kulturen. Biochem. Z. 202: 164-79 (1928).

Bernhauer, K., and Schön, K., Oxydationen mittels Bacterium xylinum. I. Die Bildung von Dioxyaceton aus Glycerin. Z. Physiol. Chem. 177: 107-24 (1928).

Bernhauer, K., and Schön, K., Oxydationen mittels Bacterium xylinum. Z. Physiol. Chem. 180: 232-40 (1929).

Bertrand, Gabriel, Action de la bactèrie du sorbose sur les alcouls plurivalents. Compt. rend. 126: 762-5 (1898).

Bertrand, Gabriel, Action de la bactèrie du sorbose sur le sucre de bois. Compt. rend. 127: 124-7 (1898).

Bertrand, Gabriel, Action de la bactèrie du sorbose sur les sucres aldehydiques. Compt. rend. 127: 728-3I (I 898).

Bertrand, Gabriel, Sur l'oxidation de l'erythrite par la bactèrie du sorbose; production d'un nouveau sucre: l'ery thrulose. Compt. rend. 130: $1330-3$ (1900).

Bertrand, Gabriel and Nityberg, Georges. Prèparation par la bactèrie du sorbose d'un nouveau sucre rèducteur á 7 atomes de carbone. Compt. rend. 186: 925-8 (I928).

Bertrand, Gabriel and Sazerac, R., Sur une différenciation biochimique des deux principaux ferments du vinaigre. Compt. rend. 132: 1504-7 (I9OI).

Binder-Kotrba, G., Über die Vergärung der Phenylglyoxylsäure. Biochem.Z. 174: 440-2 (1906).

Binder-Kotrba, G., Über die Dismutation des Acetaldols durch Bacterium ascendens. Biochem. Z. 174: 448-5I (I926).

Boas, Friedrick, Mykologischen Notizen. Centr. Bakt. Parasitenk., II. Abt., 44: 695-701 (I9I6).

BoAs, Friedrick, Untersuchungen über Säurewirkung und Bildung löslicher Stärke bei Schimmelpilzen. Centr. Bakt. Parasitenk., II. Abt., 56: 7-II (I922).

Boeknout, F. W. J., Über Dextranbilder. Centr. Bakt. Parasitenk., II. Abt., 6: 16I-5 (ig00).

Boutroux, L., Sur une fermentation nouvelle du glucose. Compt. rend. 91: 236-8 (1 880).

Boutroux, L., Sur une fermentation acide du glucose. Compt. rend. 102: $9^{24-7}$ (I 886). 
Boyland, Eric., Phosphoric esters in alcoholic fermentation. I. The sequence of the formation of phosphoric esters and carbon dioxide in fermentation by dried yeast. Biochem. f. 23: 21929 (1929).

BrAAK, H. R., Onderzoekingen over Vergisting van Glycerine. Thesis, Delft, (I928).

Breden, C. R., Fulmer, E. I., Werkman, C. H., and Hixon, R. M., The chemical action of Aerobacter faeni on xylose and on sucrose. J. Bact. 19: + I (I930).

Brieger, L., Ueber Spaltungprodukte der Bacterien. Z. physiol. Chem. 8: 306-3I I (I883).

Brit. Patent 248, 795. (See U. S. i,602,306) Fermenting cellulosic materials. Sept. 10, 1924.

Brit. Patent 249,833. (See U. S. I, 582,408) Butyl alcohol and acetone by fermentation. March 30, 1925 .

Brit. Patent 25I, 678. Alcohols and acetone by fermentation. Jan. I0, 1925 .

Brit. Patent 268,749. Butyl alcohol and acetone by fermentation. April 3, 1926.

Brit. Patent 269,950. Dihydroxyacetone. April 26, I926.

Brit. Patent 291,146. Yeast. Feb. 24, 1927.

Brit. Patent 29I,4+3. Promoting action of enzymes. June 3, 1927. Brit. Patent 293,5I4. Alcohol and acetone from straw, grasses and like materials. April 30 , 1927.

Brit. Patent 293,015. Fermentation processes. June 30, 1927.

Brit. Patent 302,338. Citric acid. Dec. 16, 1927.

Brit. Patent 306,138. Butyl acetonic fermentation of carbohydrates. Feb. 17, 1928.

Brown, A. J., The chemical action of pure cultivations of B. aceti. J. Chem. Soc. 49: 172-87 (1886).

Brown, A. J., On an acetic ferment which forms cellulose. J. Chem. Soc. 49: $432-9(1886)$.

Brown, A. J., Further notes on the chemical action of Bacternum aceti. J. Chem. Soc. 51: 638-42 (I887).

Brown, H. C. Duncan, J. 'T., and Henry, 'T. A., The fermentation of salts of organic acids as an aid to the differentiation of bacterial types. J. Hyg. 23: I 22 (1924).

Buchner, E. und Gaunt, R., Leber die Essiggärung. Liebigs Ann. 349: $140-84(1906)$.

Buchner, E., und Gaunt, R., Neue Versuche über die Oxydase der Essigbakterien. Wochenschr. f. Brau. 22: 709-10 (I906). 
Buchner, E., and Meisenheimer, Jakob., Die chemischen Vorgänge bei der alksholischen Gärung. III. Mitteilung. Ber. 39: 320 II 8, (I g06).

Buchner, Edward, and Meisenheimer, Jakob, Über Buttersäuregärung. Ber. 41: I +10-I9 (I908).

Buchner, E., and Wüstenfeld, H., Über Citronensäuregärung durch Citromyceten. Biochem. Z. 17: $395^{-4+2}$ (I909).

Butkewitsch, W., Über die Bildung der Citronen-und Oxalsäure in den Citromyces. Kulturen auf Zucker und das Verfahren zur quantitativen Bestimmung dieser Säuren. Biochem. Z. 131: 327-37 (1922).

Butkewitsch, W., Über den Verbrauch und die Bildung der Citronenšäure in den Kulturen von Citromyces glaber auf Zucker. Biochem. Z. 131: 338-50 (1922).

Butkewitsch, W., Über die Citronensäuregärung. Biochem. Z. 142: 195-2II (I923).

Butkewitsch, W., Über die Umwandlung der Chinsäure durch die Pilze. Biochem. Z. 145: 442-60 (I924).

Butkewitsch, W., Über die Bildung der Glucon-und Citronensäure in den Pilzkulturen auf Zucker. Biochem. Z. 154: I77-90 (I924).

Butкewitsch, W., Über die Säurebildung bei den Pilzen. Biochem. Z. 182: 99-109 (1927).

Cambier, R., and Auber, E., Culture de bactèries en milieu chimiquement défini à base d'acide pyruvique. Degradation de l'acide pyruvique. Compt. rend. 175: 7 I-3 (I922).

Can. Patent 285,399. Glycerol. Dec. it, 1928.

Challenger, F., The production of citric acid by fermentation process. Ind. Chemist, 5: I 8I-4 (I929).

Challenger, F., Theoretical and technical advances in the study of alcoholic fermentation. Ind. Chemist, 5: 239-42 (1929).

Challenger, F., and Klein, I., Formation of l-malic acid from fumaric acid by Aspergillus niger. J. Chem. Soc., I644-7 (1929).

Challenger, F., Subramaniam, V., and Walker, T. K., Mechanism of the formation of citric and oxalic acids from sugars by Aspergillus niger. I. J. Chem. Soc., 200-8 (1927).

Chowrenko, M. A., Über die Reduktionsvermögen der Hefe. Hydrogenisation des Schwefels bei der Alkoholgärung. Z. physiol. Chem. 80: 253-73 (1912).

Chrzaszcz, T., and Tuikow, ${ }^{8}$ D., Über die Säurebildung der Penicilliumarten. Biochem. Z. 204: 106-24.(1929). 
Cohen, Clara, Über die Bildung von Acetaldehyde bei den Umsetzungen von Zucker durch Pilze. Biochem. Z. 112: I39-43 (I 920$)$.

Conant, James B., and Aston, John G., Certain new oxidation reactions of aldehydes. J. Am. Chem. Soc. 50: $27^{8} 3^{-9} 9^{8}$ (1928). Connstein, W., and Ludecke, K., Gewinnung Glycerine durch Gärung. Ber: 52B: 1385-9I (I9I9).

Cook, Robert P. and Stephenson, Marjory. Bacterial oxidations by molecular oxygen. I. The aerobic oxidation of glucose and its fermentation products in its relation to the viability of the organism. Biochem. J. 22: 1368-86 (1928).

Coolhans, C. Thermophilic fermentation processes. Ned. Tijdschr. Hyg. Microbiol. Serol. 1: 338-50 (1926). After Chem. Absts. 21: 2009 (1927).

Coolmaas, C., Zur Kenntnis der Dissimilation fettsäurer Salze und Kohlenhydrate durch thermophile Bakterien. Centr. Bakt. Parasitenk., II Abt., 75: 34-60 (1928).

Currie, J. N., The citric acid fermentation of Aspergillus niger. J. Biol. Chem. 31: 15-37 (1917).

DAKIN, H. D., The formation of I-malic acid as a product of alcoholic fermentation by yeast. J. Biol. Chem. 61: 139-45 (1924).

Dakin, H. D., and Dudley, H. W., On glyoxalase. J. Biol. Chem. 14: 423-31 (1913).

DAY, F. E., and BAKER, J. L., A bacterium causing ropiness in Beer. Centr. Bakt. Parasitenk., II. Abt., 36: 433-8 (1913).

Delbrück, M., Die Anwendung der Enzymforschung auf die Essiggärung. Die deutsche Essigind. 7: 342 ( 1903 ).

Donker, H. J. L., Bijdrage tot de kenniss der boterzuurbutyl alcohol, -en acetongistingen. Thesis, Delft, (1926).

Effront, J., Le chemisme de la fermentation en mileu constant. Petit J. Brasseur 34: 1619-29 (1926). After Chimie et Industrie., 19: 515 (1928).

Ehrlich, Felix, Über die Entstehung der Bernsteinsäure bei der alkoholischen Gärung. Biochem. Z. 18: 39 I-423 (I909).

Ehrlich, Felix, Über die Bildung von Fumarsäure durch Schimmelpilze. Ber. 44: 3737-42 (1912).

Elfving, Fredr., Über die Bildung organischer Säuren durch Aspergillus niger. Physiol. Abstracts, 6: 162 (1919).

Emmerling, Oskar, Butylalkoholische Gärung. Ber, 30: 4513 (1897). 
Emmerling, Oskar, Über Schimmelpilzgärung. Ber. 30: $454^{-5}$ (I897).

Emmerling, Oskar, Über Spaltpilzgarüngen. Ber. 32: 19i5 -8 (1899).

Emmerling, Oskar, Der Sorbose Bakterium. Ber. 32: $54^{\mathrm{I}-2}$ (I 899).

Emmerling, Oskar, Über Spaltpilzgärungen. Ber. 33: $2477-9$ (IgO0).

Emmerling, O., and Abderhalden, E., Über einen chinasäure in Protokatechusäure überführenden Pilz. Centr. Bakt. Parasitenk. II. Abt. 10: 337-9 (1903).

Euler, Hans v., Über die Darstellung von Kohlenhydratphosphorsäurester (Zymophosphate) durch lebende Hefe. Biochem. Z. 86: $337^{-42}$ (I9I8).

Euler, Hans v., Brunius, Edvard, and Proffe, Stig. Zur Kenntnis des Aktivators $Z$. IV. Über einem spezifischen Beschleuniger der Gärung frischer Hefe. Z. physiol. Chem. 178: 202-8 (1928)

Euler, Hans v., and Myrbäck, K., Fermentation problems. Z. physiol. Chem. 181: 1-I 4 (1929). After Chem. Absts. 23:245I (1929).

Falck, R., and Kapur, S. N., Über Gluconsäure-Bildung durch Fadenpilze. Ber. 57B: 920-3 (1924).

Fernbach, A., and Schoen, M., L'acide pyruvique, produit de la vie de la levure. Compt. rend. 157: I $478-80$ (1913).

Fernbach, A., and Schoen, M., Nouvelles observations sur la production de l'acide pyruvique par la levure. Compt. rend. 158: I $79^{-22}$ (I914).

Fernbach, A., and Schoen, M., Nouvelles observations sur la productions biochemique de l'acide pyrurique. Compt. rend. 170: $764-6$ ( I 920$)$.

Fernbach, A., and Schoen, M., Se forme-t-il de l'acide lactique dans la fermentation alcoolique? Compt. rend. soc. biol. 89: $475^{-7}$ (1923).

Filosofov, M. S., and Malinovski, V. E., Citric acid fermentation, Nauchnuie Zapiski (Russian) 5: 235-9 (1928). After Chem. Absts. 21: 34 I 8 (1927).

Fitz, Albert, Über Alkoholische Gärung durch Mucor mucedo. Ber. 6: $48-58$ (1873).

Fitz, Albert, Über die Gärung des Glycerins. Ber. 9: $1348-52$ (1876).

Fitz, Albert, Über alkoholische Gärung. Ber. 9: I352-5 (1876). 
Fitz, Albert, Über Schizomyceten-Gährungen. III. Ber. 11: $4^{2-}$ 55 (I 878$)$.

Fitz, Albert, Über Spaltpilzgährungen. Ber. 13: 1309-12 (1880). Fitz, Albert, Über Spaltpilzgährungen. VII. Ber. 15: $867-80$ (1882).

Fitz, Albert, Ueber Spaltpilzgährungen. Ber. 17: i 188 (1884).

Folpmers, T., Ontleding van Koolhydraten door Granulobacterium butylicum (Beyerinck). Tijdschr, vergelyk Geneeskunde 6: $33-40$ (I921).

Frankland, Percy, and Fox, Joseph J., On a pure fermentation of mannitol and glycerin. Proc. Roy. Soc. (London) 46: $3+5^{-57}$ (1889).

Frankland, Percy, Stanley, Arthur, and Frew, William. Fermentations induced by the Pneumococcus of Friedländer. J. Chem. Soc. 59: 253-70 (1891).

Frankland, Percy, and Frew, William. A pure fermentation of mannitol and dulcitol. J. Chem. Soc. 61: 25-77 (1892).

Frankland, Percy, and Lumsden, J. S., Decomposition of mannitol and dextrose by the Bacillus ethaceticus. Chem. Soc. Trans. $61:+32-44(1892)$.

Frankland, Percy, and Macgregor, J. M., The fermentation of arabinose by Bacillus ethaceticus. J. Chem. Soc. $61: 737^{-45}$ (1892).

Franzen, H., and Egger, F., Beiträge zur Biochemie der Mikroorganismen. VI. Über die Vergärung der Ameisensäure durch Bacillus prodigiosus in konstant zusammengesetzten Nährboden. Z. physiol. Chem. 79: 177-2I+ (I912).

Franzen, H., and Kahi.enberg, H., Über die Bildung und Vergärung von Ameisensäure durch Bacterium coli. Z. physiol. Chem. 97: $314^{-2}+(1916)$.

Franzen, H., and Steppunn, O., Beiträge die Vergärung und Bildung der Ameisensäure durch Hefen. Z. physiol. Chem. 77: $129-82$ (1908).

Fred, E. B., and Peterson, IV. H., The fermentation of xylose by bacteria of the aerogenes, paratyphoid B. and typhoid groups. J. Infect. Dis. 27: $539^{-49}$ (1920).

Fred, E. B., Peterson, W. H., and Anderson, J. A., The characteristics of certain pentose-destroying bacteria, especially as concerns their action on arabinose and xylose. J. Biol. Chem. 48: $385+$ II (I 921 I).

Fred, E. B., Peterson, W. H., and Anderson, J. A. The fermentation of arabinose and xylose by certain aerobic bacteria. J. Bact. 8: $277-86(1923)$. 
Fred, E. B., Peterson, W. H., and Davenport, Audrey, Acid fermentation of xylose. J. Biol. Chem., 39: 347-8+ (I9I9).

Fred, E. B., Peterson, IV.H., and Davenfort, Audrey, Fermentation characteristics of certain pentose-destroying bacteria. J. Biol. Chem. 42: 175-89 (1920).

Fred, E. B., Peterson, IW. H., and Stules, H. R., 'The biochemistry of the granulated lactic acid bacteria from cereals. J. Bact. 10: $62-78$ (1925).

French Patent 636,121. Glycerol by fermentation. June 17, 1927. After Chem. Absts.

French Patent 643,996. Vinegar. Nov. 2, 1927. After Chem. Absts. Freudenreich, E. von and Jensen, Orla-, Über die im Emmentalerkäse stattfindende Propionsäuregärung. Centr. Bakt. Parasitenk. Abt. II, 17: 529 (I906)

Frings, H., Essigbakterien-Dauerkulturen mittels des FringsschenDauerkultur-Kolbens. Die deutsche Essigind. 17: I I4-5 (I9I3).

Gayon, U. and Dubourg, E., Sur la fermentation alcoolique de la dextrine et de l'amidon. Compt. rend. 103: 885-7 (I 886).

Gehle, Heinrich, Vergärung von Zucker bei Gegenwart von Dinatriumsulfit nach Neuberg und Reinfürth. Nachprüfung der Äquivalenz zwischen Aldehyd und Glycerin. Biochem. Z. 132: 566-88 (1922).

Gillot, Henri, La Raffinose considérée hydrocarboné de l'Aspergillus niger. Bul. Acad. Roy. Belg., 1899: 2 I I-26.

Glimm, E. and Grimm, R., Salzhydrolyse der Stärke. Biochem. Z. 197: 445-59 (1928).

Golovin, P. V., Glycerol from molasses. Zhur. Sakharnoi Prom. 1: 22-5 (1927). After Chem. Absts.

Gorr, Günther, and Perlmann, Genia, Weitere Untersuchungen über die Bildung von Milchsäure aus Methylglyoxal durch Ketonaldehydmutase tierischer und pflanzlicher Herkunft. Biochem. Z. 174: $433-9$ ( 1926$)$.

Gото, GüchI, Über die Glykolitische Wirkung des Bacterium coli commune. Sci. Rpts. Gov't. Inst. for Infect. Diseases. Tokyo Imp. U. 4: 367 (I925).

Gottschalk, A., Über die carboxylatische Spaltung der Brenztraubensäure in Säuerstoffstrom. Biochem. Z. 140: 348-52 (1923). Gotтschalk, A., Aufbau und Vergärung von Glycogen durch Maltosefreie Hefe. Z. physiol. Chem. 152: 132-5 (1925). Gоттsсна LK, A., Biochemische Synthese von Fumarsäure aus Brenztraubensäure. Z. physiol. Chem, 152: 136-43 (1925). 
Gotтschalk, A., Weitere Untersuchungen über Hefeamylase. Z. physiol. Chem. 178: I39-47 (I928).

Gotтschalk, A., Nochmals zur Frage der biochemischen Fumarsäüresynthese aus Brenztraubensäure. Z. physiol. Chem. 182: $311^{-2}$ (1929).

Graaf, W. C. De, The Mixed Acid Fermentation. A contribution to the fermentative sugar dissimilation by microorganisms of the coli group. Nederland. Tijdschr. Hyg. Microbiol. Serol. 1: $43-70$ (1926). After Chem. Absts.

GraAf, W. C. De and Le Fèvre, A. J., Beiträge zur Kenntnis der bakteriellen Gärungen insbesondere in der Koli-TyphusGruppe. Biochem. Z., 155: 313-332, 1925.

Gray, P. H. H., Formation of indigotin from indole by soil bacteria. Proc. Roy. Soc. London (B) 102: 263-80 (1928).

Grey, E. C., The production of acetaldehyde during the anaerobic fermentation of glucose by Bacillus coli communis (Escherich). Biochem. J. 7: 359-64 (1913).

Grey, E. C., The enzymes concerned in the decomposition of glucose and mannitol by Bacillus coli communis. Part. II. Experiments of short duration with an emulsion of the organisms. Proc. Roy. Soc. London (B) 90: 75-92 (1918).

Grey, E. C., The enzymes concerned in the decomposition of glucose and mannitol by Bacillus coli communis. Part III. Various phases in the decomposition of glucose by an emulsion of the organisms. Proc. Roy. Soc. London (B) 90: 92-106 (1918).

Grey, E. C., The enzymes of B. coli communis which are concerned in the decomposition of glucose and mannitol. Part IV. The fermentation of glucose in the presence of formic acid. Proc. Roy. Soc. London (B), 91: 294-305 (1920).

Grey, E. C., The latent fermentating powers of bacteria I-III. I. The fermentation of glycerin by coliform organisms in the presence of formates. II. The fermentation of glycol and of malonic, malic, tartaric, and citric acids by B. coli communis in the presence of calcium formate: with the preliminary notice of a simple theory of fermentation. Proc. Roy. Soc. London (B) 96: 156-69 (1924).

Grey, E. C., The synthesis of starch from sugar by bacteria. Biochem. J. 18: 712-5 (1924).

Grey, E. C., and Young E. G., The enzymes of B. coli communis. Part $V$. (a) Anaerobic growth followed by anaerobic and aerobic fermentation. (b) The effects of aeration during fermentation. Proc. Roy. Soc. London (B) 92: I35-50 (I921). 
Grimbert, M. L., Fermentation anaerobie produite par Bacillus orthobutylicus. Ann. Inst. Pasteur, 7: 353-402 (I 893).

Grimbert, L., Action du pneumobacille de Friedlaender sur la xylose et l'arabinose. Compt. rend. soc. biol. 48: ig I-2 (1896).

Grimbert, L., Coli-bacille produisant de l'acide succinique avec le lactose. Compt. rend. soc. biol. 48: 192-5 (1896).

Grimbert, L., Production d'acetylmethylcarbinol par le Bacillus tartricus. Compt. rend. 132: 706-9 (I 901).

Grimbert, L., and Ficquet, J., Sur un nouveau ferment des tartrates, le Bacillus tartricus. J. pharm. chim. 7: 97-100 (1898).

Groenewege, J., Weitere Untersuchungen über den eigentlichen âeroben, sowie über den Denitrification Process. Botan. Abstracts 14: I22 (1923).

Guittonneau, G., Sur un bacille sporulé thermophile fonctionnant comme ferment lactique. Compt. rend. 187: $69-72$ (I928).

Hägglund, Erick, and Ӓнвом, L., Vergleichende Untersuchungen über die Vergärung von Glucose und Brenztraubensäure. Biochem. Z. 181: 158-71 (1927).

Hammer, B. W., The type of lactic acid produced by starters and by organisms isolated from them. Res. Bull. 65. Iowa Agric. Exp. Sta. (1920).

Hammer, B. W., Volatile acid production of $S$. Lacticus and the organisms associated with it in starters. Res. Bull. 63. Iowa Agric. Exp. Sta. (1920).

Hammer, B. W., and Baker, M. P., Classification of the Streptococcus lactis group. Res. Bull. 99. Iowa Agric. Exp. Sta. (I926).

Hammer, B. W., and Sherwood, F. F., The volatile acids produced by starters and organisms isolated from them. Res. Bull. 80 . Iowa Agric. Exp. Sta. (1923).

Hansen, Emil Chr., Botanische Untersuchungen über Essigsäurebacterien. Ber. Bot. Ges., 11: (69)-(73). (1893).

Hansen, E. C., Recherches sur les bactéries acétifiantes. Compt. rend. Lab. Carlsberg, 5: 39-46 (I900).

Hansen, Emil Chr., Gesammelte theoretische Abhandlungen über Gärungorganismen, Jena (I9II).

Harden, Arthur, Fermentation of sugars by B. coli communis and allied organisms. Trans. of Jenner Inst. of Prevent. Med. 2: I 26 (I 899).

Harden, A., and Young, IV. J., The alcoholic ferment of yeastjuice. Proc. Roy. Soc. B. 77: 405-20 (1905). 
Harden, A., and Young, W. J., The alcoholic ferment of yeast-juice. V. The function of phosphates in alcoholic fermentation. Proc. Roy. Soc. Lond. B. 82: 321-30 (I9I0).

Harden, Arthur, The chemical action of $B$. coli communis and similar organisms on carbohydrates and allied compounds. J. Chem. Soc. 79: 610-28 (I901).

Harden, Arthur, and Norris, Dorothy, The bacterial production of acetylmethylcarbinol and 2, 3-butylene glycol from various substances. Proc. Roy. Soc., London, (B) 85: 73-8 (I912).

Harden, Arthur and Henley, Francis R., The equation of alcoholic fermentation. II. Biochem. J. 23: 230-6 (I929).

Harden, Arthur and Norris, Dorothy, The bacterial production of acetylmethyl carbinol and 2, 3-butylene glycol from various substances. Proc. Roy. Soc. London, (B) 84: +92-9 (I9I2).

Harden, Arthur and Walpole, George Stanley, Chemical action of Bacillus lactis aerogenes (Escherich) on glucose and mannitol: production of 2, 3-butylene glycol and acetylmethyl carbinol. Proc. Roy. Soc. London, 77 B: 399-4ว5 (I906).

Harden and Young, The alcoholic ferment of yeast-juice. Proc. Roy. Soc. Lond. B. 77: 405-20 (I 906).

Heinze, Berthold, Einiges ueber Säurebildung durch Pilze, insbesondere auch über Essigsäure-und Oxalsäurebildung durch Aspergillus niger. Ann. Mycologici, 1: 344-53 (1903).

Henneberg, W., Beiträge zur Kenntnis der Essigbakterien. Centr. Bakt. Parasitenk. II. Abt., 3: 223-8 (1897).

Henneberg, W., Weitere Untersuchungen über Essigbakterien. Centr. Bakt. Parasitenk., II Abt. 4: 67-73, I38-47 (1898).

Henneberg, W., Zur Kenntnis der Milchsäurebakterien der Brennereimaische, der Milch des Bieres, der Presshefe, der Melasse, des Säuerkohls, der Säuren Gurken, und des Säurteigs, sowie einige Bemerkungen über die Milchsäurebakterien des menschlichen Magens. Z. Spiritusind. 26: 277-9 (1903).

Henneberg, W., Gärungsbakteriologisches Praktikum, Betriebsuntersuchungen und Pilzkunde. Paul Parey, Berlin. (1909). Henneberg, W., Handbuch der Gärungsbakteriologie. Berlin, Paul Parey (1927).

Henninger, and Sanson, Présence d'un glycol dans les produits de la fermentation alcoolique du sucre. Compt. rend. 106: 208-10 (I 888 ).

Hermann, S., Ueber die Bildung von Gluconsaure und Keto-gluconsäure durch Bacterium gluconicum, Bacterium xylinum und Bacterium xylinoides. Biochem. Z. 214:357-67 (I929). 
Herrick, Horace T., and May, Orville E.. The production of gluconic acid by the Penicillium luteumpurpurogenum group. II. Some optimal conditions for acid formation. J. Biol. Chem. 77: I 85-95 (1928).

Herrick, H. T., and May, O. E., Molds and chemical manufacture. Ind. and Eng. Chem. 21: 618-21 (1929).

Herzog, R. O., and Ripke, O., Notiz über die Umwandlung von Zimtsäure in Styrol durch Schimmelpilze. Z. physiol. Chem. 57: $43^{-5}(1908)$.

Hewitt, James Arthur and Steabben, Dorothy B., Note on the fermentation of i-inositol. Biochem. J. 15: 665-6 (I92I).

Hildebrandt, F. M., Adjustment of acidity of cane-molasses fermentation for maximum alcohol yields. Ind. Eng. Chem. 21: 779-8I (I929).

Hirsch, Julius, Ủber eine biosynthetische Kohlenstoffkettenverknüpfung in der aliphatischen Reihe. V. Zur Kenntnis der Carboligasse. Biochem. Z. 131: I78-87 (1922).

Hoyer, Kirk Pieter, Bijdrage tot de Kennis van de Azijnbacteriën Thesis Leiden. After Centr. Bakt. Parasitenk., II Abt. 4: $867-75,(1898)$.

Hucker, G. J., Studies on the Coccaceae. XIV. Certain biochemical reactions produced by the streptococci. Tech. Bull. N. Y. Agr. Expt. Sta. 143: I-64 (1928).

Humphreys, Frederick B., Formation of acrolein from glycerol. J. Infect. Dis., 35: 282-90 (1924).

Iwanoff, Leonid, Über die Bildung der phosphororganischen Verbindungen und ihre Rolle bei der Zymasegärung. Centr. Bakt. Parasitenk. II Abt. 24: I-I2 (I909).

IwASAKI, Ken, Über den Mechanismus der Vergärung des Dioxyacetons. Biochem. Z. 203: 237-66 (I928).

Iwatsuru, Rynzo, Über die Vergärung der $\alpha$-Ketoglutarsäure durch das Bacterium xylinum. Biochem. Z. 168: 34-5 (1925).

Janke, A., Die Säuerung des Äthylalkohols durch Essigsäure-Bakterien. Centr. Bakt. Parasitenk. II. Abt., 45: $534^{-47}$ (I9I6). Jankovi, Svetozar, Influence of certain factors on formation of empyreumatic oil by fermentation of molasses. Arhic. Hemiju Farm. 1: 218-26 (1927). After Chem. Absts. 22: 2233 (1928). Kageura, Naomi, Einwirkung des Bacteriums lactis aerogenes und des Bacteriums coli auf Hexose-mono-phosphorsäure. Biochem. Z. 190: 181-7 (1927). 
Karber, P. and Hofmann, A., Polysaccharides. XXXIX. The enzymic degradation of chitin and chitosan. Helv. Chim. Acta. 12: 616-37 (1929). After Chem. Absts. 22: 2055. (1929).

Karczag, L., In welcher Weise wird die Weinsäure durch Hefe angegriffen? Biochem. Z. 43:43-6 (I9I2).

Karczag, L., and Móczár, L., Über die Vergärung der Brenztraubensäure durch Bakterien. I. Biochem. Z. 55: 79-87 (I9I3).

Kay, Herbert Davenport, Note on the variation in the end products of bacterial fermentation resulting from increased combined oxygen in the substrate. Biochem. J. 20: 320-9 (1926).

Kayser, E., Action de la levure sur le lactate de chaux, production d'alcool éthylique. Compt. rend. 176: 1662-5 (1923).

Kerry, Richard and Frankel, Sigmund, Über die Einwirkung der Bacillen des malignen Oedems auf Kohlenhydrate. Monatsh. 11: 268-7 I (I 890).

Khouvine-Delaunay, K., Un anaerobie de l'untestine humain digerant la cellulose. Compt. Rend. Soc. Biol., 87: 922-3 (1922).

Killeffer, D. H., Butanol and acetone from corn. Ind. Eng. Chem. 19: $46-50($ I 927$)$.

Kimura, H., The formation of optically active lactic acid from glucose and dl-lactic acid by bacteria. Univ. Kyoto Acta. Schol. Med. Univ. Imp. Kiota 11: 325-33 (1928).

Kinoshita, K., Growth of molds on cobaltammine salts. Acta Phytochim. 3: 3I-50 (I927).

Klein, Gustav and Fuchs, Walter, Zum Brenztraubensäurenachweis bei der Hefe Gärung. Biochem. Z. 213: 40-64 (1929). Kling, Andre, Oxidation du propylglycol par le Mycoderma aceti. Compt. rend. 133: 231-3 (IgOI).

Kling, A., Contribution a l'étude des alcools cétoniques. Ann. de Chimie et de Phys. 8 Série, 5: $47^{\mathrm{I}-3}$ (I905).

Kluyver, A. J., Ueber eine neue Theorie des Mechanismus der alkoholischen Gärung. Wochschr. Brau. 46: 66-70 (1929).

Kluyver, A. J., and Donker, H. J. L., The formation of acetyl methyl carbinol and 2, 3-butylene glycol in the course of sugar fermentations by alcohol, yeasts and true lactic acid bacteria. Verslag. Akad. Wetenschappen Amsterdam, 33: 915-9 (1924); Proc. Acad. Sci. Amsterdam 28: 3I $4-7$.

Kluyver, A. J., Donker, H. J. L., and Hooft, F. Visser't., Über die Bildung von Acetylmethyl carbinol und 2, 3-Butylene-glykol im Stoffwechsel der Hefe. Biochem. Z. 161: 36I-78 (1925). 
Kluyver, A. J., and de Leeuw, F. J. G., Acetobacter suboxydans, een merkwaardige Azijnbacterie. Tijdschr. vergljk. Geneeskunde 10: $170-83$ (1924).

Kluyver, A. J., and Struyk, A. P., De eerste phasen van het chemisme der dissimilatie der hexosen. Verslag Akad. Wetenschappen Amsterdam 36: 608-2 I (1927).

Kluyver, A. J., and Struyk, A. P., Ueber die ersten chemischen Phasen des Hexosen-Abbaues bei der alkoholischen Gärung. Wochschr. Brau. 46: 5I-5 (I929).

Knieriem, W. v. und Mayer, Adolf, Ueber die Ursache der Essiggährung. Die Landwirthschaftlichen Versuchs-Stationen, 15: 305-29 (1872).

KöNIG, F., Über Gährung der Weinsäure. Ber. 14: 21 I-7 (I 88I). Kostyтschew, S., Über die Alkoholgärung von Aspergillus niger. Ber. Botan. Ges. 25: 44-50 (1907).

Kostytschew, S., Über Alkoholgärung. I. Mitteilung. Über die Bildung von Acetaldehyd bei der Alkoholischen Zuckergärung. Z. physiol. Chem., 79: 130-45 (1912).

Kostytschew, S., Über Alkoholgärung. VI. Mitteilung. Das Wesen der Reduction von Acetaldehyd durch lebende Hefe. Z. physiol. Chem. 89: 367-72 (I9I4).

Kostytschew, S., and Afanassjewa, M., Die Verarbeitung verschiedener organischer Verbindungen durch Schimmelpilze bei Säuerstoffmängel. Jahrb. wiss. Botanik. 60:628-50 (1922).

Kostytschew, S., and Chesnokov, V., Zitronensäure und Oxalsäure durch Aspergillus niger, Planta. Archiv. f. Wiss. Bot. Abt. E. 4: I 8 I-200 (1927).

Kostytschew, S., and Eliasberg, P., Über Alkoholgärung. X. Mitteilung. Gärung ist Leben ohne Sauerstoff. Z. physiol. Chem. 111: I $_{4}{ }^{\mathrm{I}-56}$ (1920).

Kostytschew, S., and Faermann, V., Über Alkoholgärung. XIV. Die Verarbeitung der mehrwertigen Alkohole durch Hefe. Z. physiol. Chem. 173: 72-9 (I928).

Kostytschew, S., and Frey L., Über Alkoholgärung. XI. Mitteilung Über die bei der Hefegärung in Gegenwart von Calcium Carbonat entstehenden Säuren. Z. physicl. Chem. 146: 276-85 (1925).

Kostytschew, S., and Hüввеnet, E., Über Alkoholgärung. II. Mitteilung. Über Bildung von Äthylalkohol aus Acetaldehyd durch lebende und getötete Hefe. Z. physiol. Chem. 79: 359-74 (I9I2). 
Kostytschew, S. and Hübbenet, E., Zur Fräge der Reduktion von Acetaldehyd durch Hefesaft. Z. physiol. Chem. 85: 408-I I (1913).

Kostytschew, S., and Shulgina, O., Über Alkoholgärung. XIX. Über einige gärungsfähige Mikroben im Macerationssaft. Z. physiol. Chem. 182: 50-6 (I929).

Kostytschew, S., and Soldatenkov, S., Wichtige Zwischenproduckte der alkoholischen und Milchsäure- Gärung abgefangen. Wochschr. Brau. 44: $4^{\mathrm{I}} 4^{-5}$ (I927).

Kostytschew, S., and Jegorova, K., Über Alkoholgärung. XVIII. Das Verhalten von Hefe gegenüber Glycerinaldehyd und Glycerinsäure. Z. physiol. Chem. 181: 26+-80 (1929).

Kostytschew, S., and Soldatenkov, S., Brenztraubensäure und Methylglyoxal als Zwischenprodukte der Milchsäuregärung. Z. physiol. Chem. 168: I 24-7 (I927).

Kostytschew, S., and Soldatenkor, S., Über Alkoholgärung. XVII. Brenztraubensäure als ein intermediares Product der alkoholischen Hefegärung. Z. physiol. Chem. 176: 287-9 (1928).

Kumagawa, H., Erzielung der zweiten und dritten Vergärungsform mit Saccharomyces Sake, Zygosaccharomyces major und Zygosaccharomyces salsus. Biochem. Z. 131: I $48-56$ (I922).

Kumagawa, H., Über die Zerlegung des meso-Inosits und Glycerins nach Art der wahren Zucker durch den Bacillus lactis aerogenes. Biochem. Z. 131: I 57-60 (1922).

Lander, C. H., et al., Power alcohol from grasses, straws and waste vegetable materials. Dept. Sci. Research (Fuel Research) Fuel for Motor Transportation, th Mem., 26 pp. (1927).

Lebedev, A. N., Fermentation of dibasic acids. I. Fermentation of malic acid. J. Russ. Phys. Chem. 48: 725-48 (I9I6).

Lebedev, A. N., Sur la fermentation de l'acide glyoxylique. Biochem. J. $12: 8 \mathrm{I}-6(1918)$.

Lebedev, A. N., Über die zellfreie Gärung. Z. physiol. Chem. 173: $89-102$ (1920).

Lebedev, A. N., Über den Mechanismus der alkoholischen Gärung. Biochem. Z. 200: 149-5 I (1928).

Lebedev, A. N., and Polonski, A. N., Fermentation of glyceric acid and pyruvic acid. J. Russ. Phys. Chem. Soc. 49: $344^{-57}$ (1917).

Lebedev, A. N., and Polonski, A. N., Fermentation of glyceric and pyruvic acids. J. Russ. Phys. Chem. Soc. 49: 93-4 (1917). 
Lebedev, A. N., and Polonski, A. N., Fermentation of pyruvic acid in the presence of sugar. J. Russ. Phys. Chem. Soc. 49: 328-44 (1917).

LeBel, J. A., Sur le propylglycol actif. Compt. rend. 92: 532-4 (I88I).

Lemoigne, M., Fermentation du sucre par le Bacillus subtilis. Production de 2-3, butyléneglycol. Compt. rend. 155: 792-5 I9I 2).

Lemoigne, M., Assimilation du saccharose par les bactèries du groupe du "B. subtilis." Fermentation butylèneglycolique. Ann. Inst. Pasteur 27: 856-85 (I9I3).

Lemoigne, M., Fermentation butyléneglycolique des sucres par la bactèridie charbonneuse. Compt. rend. soc. biol. 82: $9^{8} 4^{-6}$ (I9I9).

Lemoigne, M., Fermentation butyléneglycolique du glucose par certaines bactèries du groupe du Bacillus proteus. Compt. rend. soc. biol. 88: $4^{6} 7^{-8}$ (1923).

Lemoigne, M., Fermentation butyléneglycolique du lactate de calcium par les bactèries du groupe du B. subtilis. Compt. rend. 177: 652-4 (I923).

Lemoigne, M., Produits de déshydration et de polymérisation de l'acide $\beta$-oxybutyrique. Bull. soc. chim. biol. 8: 770-82 (I926).

Lintner, C. J. and Liebig, H. J. v., Über die Reduktion des Furfurols durch Hefe bei der Alkoholischen Gärung. Z. physiol. Chem. 72: $4+4-5+$ (I9I I).

Lintner, C. J., and Liebig, H. J., Über die Einwirkung gärender Hefe auf Furfurol Bildung von Furyltrimethyleneglykol. Z. physiol. Chem. 88: 109-2 I (I9I3).

Lintner, C. J., and Lüers, H., Über die Reduktion des Chloralhydrats durch Hefe bei der alkolischen Gärung. Z. physiol. Chem. 88: 122-3 (1913).

Loew, O., Über das Verhalten der Chinasäure an den Spaltpilzen. Ber. 14: 450-2 (I88I).

Lukow, Curt, Vom Wesen der Eissiggärung. Centr. Bakt. Parasitenk. II Abt. 72: 38-66 (1927).

Lundestadt, Jon, Über einige an der norwegischen Küste isolierte agarspaltende Arten von Meerbakterien. Cent. Bakt. Parasitenk., II Abt., 75: 32I-44 (1928).

Makrinov, I. A., Sur un nouveau Microorganisme provoquant la fermentation de l'amidon et des Matiéres Pectiques. Arch. Sci. Biol. (Petrograd) 18: 440-52 (1915). After Abstracts Bact. 7: 172 . 
Marten, E. A., Sherrard, E. C., Petersen, IT. H., and Fred, E. B., Production of lactic acid by fermentation of wood sugar remaining after alcoholic fermentation. Ind. Eng. Chem. 19: II $62-5$ (1927).

Martin, John Albert, Citric acid by fermentation. Am. J. Pharm. 88: $337-55$ ( 1916$)$.

Maumus, Sur la transformation de l'amidou vegetal en sucre par le bacille du charbon. Compt. rend. soc. biol. 45: 107-9 (1893). Maumené Théorie de la fermentation alcoolique. Compt. rend. 45: IO2I-3 (1 857$)$

Maurer, Kurt, Beobachtungen über die Zuckerspaltung durch das Bacterium propionicum. Biochem. Z. 191: 83-7 (1927).

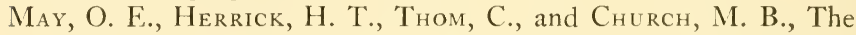
production of gluconic acid by the Penicillium luteum-purpurogenum group. I. J. Biol. Chem. 75: 417-22 (1927).

May, Orville E., Industrial mold fermentations. Hexagon of Alpha Chi Sigma 19: 1 25-30 (I928).

Mayer, Paul, Zuckerfreie Gärung bei Stereoisomeren. Biochem. Z. 50: $283^{-7}$ (1913).

Mayer, Paul, Zur Biochemie des Asymmetrieproblems. Biochem. Z. 174: $420-4$ (1926).

Mayer, Paul, and Neuberg, Carl, Phytochemische Reduktionen. XII. Die Umwandlung von Citronellal in Citronellol. Biochem. Z. 71: $174^{-9}$ (1915).

Mazé, P., Note sur la production de l'acide citrique les citromyces (Wehmer) Ann. Inst. Pasteur 23: 830-3 (1909).

Mazé, P., Fermentation alcoolique de l'acide lactique. Compt. rend. 156: IIOI-4 (I9I3).

Mazé, P., L'oxydation de l'acide lactique par les bactéries avec formation d'acide pyruvique et de composés cétoniques. Compt. rend. soc. biol. 81: I 50 (I9I8).

Mazé, P., and Ruot, M., La production de l'acide pyruvique par oxydation biochemique de l'acide lactique. Compt. rend. soc. biol. 79: 706-10 (1916).

Mazé, P., and Ruot, M., Recherches sur l'assimilation de l'acide lactique par les levures et les oidiums. Compt. rend. soc. biol. 80: 336-9 (1917).

Mazé, P., and Perrier, A., Production d'acide citrique par les citromyces. Ann. Inst. Pasteur 18: 553-75 (1904).

Meyerhof, O. Über den Einfluss des Sauerstoffs auf alkoholische Gärung der Hefe. Biochem. Z. 162: 43-86 (1925). 
Mezzadroli, G., The commercial use of saccharose-inverting bacteria for the manufacture of lactic acid, acetic acid and acetone. Bull. Agr. Intelligence 8: 787-9 (1917) from Prelim. note in Bollettino dell'assoc. ind. zucchero e dell' alcool. 9: No, IO, I $42-5$. Mezzadroli, Guiseppe, Un Leivito del Mannosio. Staz. sper. agrar. ital. 51: 306-I I (I9I8).

Molliard, Marin, Sur une nouvelle fermentation acide produit par le Sterigmatocystis nigra. Compt. rend. 174: 88I-2 (I922). Morin, Ed. Charles, Formation d'alcool amylique normal dans la fermentation de la glycerine par le Bacillus butylicus. Compt. rend. 105: 8I6-8 (I887).

Müller, D., Ein neues Enzym-Glykoseoxidase aus Aspergillus niger. Kgl. Veterinaer-og Landbohjskole Aarssakrift, 329-3I (1925).

Müller, D. Ein neues Enzym-Glykoseoxidase. Biochem. Z. 205 : I I I-43, (I 929).

Mrrback, Karl, and Jacobi, Willy, Zur Kenntniss der enzymatischen Umwandlungen der Aldehyd. II. Z. physiol. Chem. 161: 245-53 (1926).

NagaI, K., Über die Bildung von Acetaldehyd beim bakteriellen Abbau von Säuren der Kohlenhydratreihe und verwand ten Säuren. Biochem. Z. 141: 266-8 (I923).

Nagar, K., Über die Bildung von Acetaldehyd bei der Vergärung von Fructose, Galaktose, Saccharose, Maltose und Lactose durch Bacterium coli und Bacillus lactis aerogenes. Biochem. Z 141: 26I-5 (I923).

Nagayama, T., Über die Zerlegung der Brenztraubensäure durch verschiedene Pilze. Biochem. Z. 116: 303-6 (I921).

Neill, James M., and Gaspari, E. L., Studies on bacterial enzymes. I. Meningococcus maltase. J. Exp. Med. 45: I 5 I-62 (I927). Nelson, E. K., and Greenleaf, C. A., An investigation of cane mollasses distillery slop, with special reference to certain organic acids. Ind. Eng. Chem. 21: 857-9 (1929).

Nencki, M., and Sieber, N., Über die Bildung der Paramilchsäure durch Gärung des Zuckers. Monatsh. 10: 5.32-40 (1889).

Neubauer, Otto, and Fromherz, Konrad, Über die Abbau der Aminosäuren bei der Hefegärung. Z. physiol. Chem. 70: 32650 (I9II).

Nfuberg, C., Über Zuckerfreie Hefegärung. VII. Bildung von $\beta$-oxybuttersäurealdehyd (aldol) bei der Vergärung von Brenztraubensäure. Biochem. Z. 43: 49I-3 (I9I2). 
Neuberg, C., Weitere Untersuchungen über die biochemische Umwandlung von Methylglyoxal in Milchsäure nebst Bemerkungen über die Entstehung der verschiedenen Milchsäure in der Natur. Biochem. Z. 51: $4^{8} 4^{-508}$ (I9I3).

Neuberg, C., and Arinstein, B., Vom Wesen der Buttersäureund Butylalkoholgärung. Abfang von Acetaldehyd als Umsetzungsproduct. Übergang von Brenztraubensäure-aldol in Buttersäure. Entstehung höherer Fettsäuren aus Zucker. Biochem. Z. 117: 269-3I4 (I92I).

Neuberg, C., and Cohen, Clara, Über die Bildung von Acetaldehyd und die Verwirklichung der zweiten Vergärungsform bei verschiedenen Pilzen. Biochem. Z. 122: 204-24 (I92I).

Neuberg, C., and Gorr, G., Neue Untersuchungen über die Vergärung der Oxalessigsäure. Biochiem. Z. 154: 495-502 (1924). Neuberg, C., and Gorr, G., Über die Mechanismus der Milchsäurebildung bei Bakterien. Biochem. Z. 162: 490-5 (1925).

Neuberg, C., and Gorr, G., Über den Mechanismen der Milchsäurebildung bei Bakterien, II. Biochem. Z. 166: ${ }_{4} 82-7$ (1925).

Neuberg, C., and Gorr, G., Die Bilanz der Oxalessigsäuregärung. Ergebnisse Physiol. 24: I9I-5 (I925).

Neuberg, C., and Gorr, G., Über die saccharogene Bildung von Milchsäure durch verschiedene Bakterien, die Methylglyoxal dismutieren, und über eine einfache Art der I solierung von Lactat. Biochem. Z. 173: $476-8$ I (1926).

Neuberg, C., and Hirsch, Julius, Über den verlauf der Alkoholischen Gärung bei Alkalischer Reaktion. II. Gärung mit lebender Hefe in Alkalischen Lösungen. Biochem. Z. 96: 175-203 (1919).

Neuberg, C., and Kerb, Elizabeth, Phy tochemische Reduktionen. $\mathrm{XV}$. Die Überführung von Acetaldol in optischaktives $\beta$-Butylene glykol durch Hefe. (nebst Anhang: Darstellung, Reaktionen, und krystallisierte Abkämmlinge des Aldols). Biochem. Z.

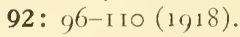

Neuberg, C., and Kerb, Elizabeth, Phytochemische Reduktionen. XVI. Die Umwandlung von Citral in Geraniol durch Hefe (nebst Anhang: Darstellung und krystallisierte Abkämmlinge des Zyklocitrals). Biochem. Z. 92: i I I-23 (I9I8).

Neuberg, C., and Kerb, J., Über Zuckerfrei Hefegärung. IX. Vergärung von Ketosäuren durch Weinhefen. Biochem. Z. 47: 405-12 (1912).

Neuberg, C., and Kerb, J., Über 'Zuckerfreie Hefegärung. X. Die Gärung der $\alpha$-Ketobuttersäure. Biochem. Z. 47: $4^{1} 3^{-20}$ (I9I2). 
Neuberg, C., and Kerb, J., Über Zuckerfreie Hefegärung. XII. Über die Vorgänge bei der Hefegärung. Biochem. Z. 53: 40621 (I913).

Neuberg, C., and Kerb, J., Zuckerfreie Hefegärungen. XV. Über die Bildung von n-Propyl-Alkohol bei der Vergärung. von $\alpha$-Ketobuttersäure. Biochem. Z. 61: 184-6 (1914).

Neuberg, C., and Ковец, M., Über das physiologische Verhalten des Acetoins. I. Mitteilung. Über das Verhalten des Acetoins zu Hefe. Biochem. Z. 160: 250-5 (1925).

Neuberg, C., and Kobel, M,. Über die Bildung von reiner d-l, -Milchsäure durch frische Hefen und Trockenhefe säure von d-1Milchsäure durch Hefenmazerationssaft. Biochem. Z. 182: $470-87$ (1927).

Neuberg, C., and Kober, M., Über die biochemische Umwandlung von Dioxyaceton in Hexosen auf dem Gärungswege und über die Vergärungsgeschwindigkeit des Di-oxyacetons im Zusammenhang mit der Verbrennungswärme dieser Triose. Biochem. Z. 203: $452-62$ (1928).

Neuberg, C., and Ковец, M., Ergänzung zur vorstehenden Veröffentlichung und $\mathrm{zu}$ unserer früheren Mitteilung über vermeintliche Abfangung von Methylglyoxal bei der alkoholischen Gärung. Biochem. Z. 199: 230-I (1928).

Neuberg, C., and Kobe1, M., Weiteres über Vorgänge bei der desmolytischen Bildung von Methylglyoxal durch Hefe. Biochem. 210: 466-88 (1929).

Neuberg, C., and Komarewsky, W., Über die Umwandlung des Methyl-benzoyl-carbinols durch gärende Hefe. Biochem. Z. 182: $285^{-90}$ (I927).

Neuberg, C., and Liebermann, Ludwig, Zur Kenntnis der Carboligase. II. Biochem. Z. 121: 3I I-25 (I92I).

Neuberg, C., and MAY, A. V., Die Bilanz der Brenztraubensäuregärung. Biochem. Z. 140: 299-313 (1923).

Neuberg, C., and Nord, F. F., Phy tochemische Reduktionen. IV.

(a) Über die Bildung von n-Amylalkohol durch Hefe. (b) Beobachtung über natürliches Vorkommen von n-Amylalkohol. Biochem. Z. 62: ${ }_{4}^{82-8}$ (I9I4).

Neuberg, C., and Nord, F. F., Phytochemische Reduktionen. VI. Bildung von n-Hexylalkohol durch Hefe. Biochem. Z. 67: $24-7$ (I9I4).

Neuberg, C., and Nord, F. F., Phytochemische Reduktionen. VII. Die Enzymatische Umwandlung des Thioacetaldehyds in Äthylmercaptan. Biochem. Z. 67: 4 6-50 (1914). 
Neuberg, C., and Nord, F. F., Phytochemische Bildung von Äthylm ercaptan. Ber. 47: 2264-7I (1914).

Neuberg, C., and Nord, F. F., Die phytochemische Reduktion der Ketone. Biochemische Darstellung optisch-aktiver secundater Alkohole. Ber. 52 B: 2237-48 (I919).

Neuberg, C., and Nord, F. F., Phytochemische Reduktion von Diketone. Ber. 52 B: 2248-54 (I919).

Neuberg, C., Nord, F. E., and Wolff, E., Acetaldehyd als Zwischenstufe bei der Vergärung von Zucker durch B. lactis aerogenes. Biochem. Z. 112: 144-50 (1920).

Neuberg, C., and Ohle, H., Zur Kenntnis der Carboligase. IV. Mitteilung. Weitere Festellungen über die Biosynthetische Kohlenstoffkettenverknüpfung Gärungsvorgänge. Biochem. Z. 128: 610-8 (1922).

Neuberg, C., and Peterson, W. H., Die Valeraldehyd-und Amylalkohol-gärung der Methyläthylbrenztraubensäure. Biochem. Z. 67: 32-45 (19I4).

Neuberg, C., and Reinfürth, Elsa, Natürliche und erzwungene Glycerinbildung bei der alkoholischen Gärung. Biochem. Z. 92: $234-66$ (I918).

Neuberg, C., and Reinfürth, Elsa, Über die Vergärbarkeit der Brenztraubensäure unter den Bedingungen des Abfangverfahrens. Vergärung der Pyruvinate-Sulfit durch Hefe. Ber. 53 B : 103952 (I 920$)$.

Neuberg, C., and Reinfürth, Elsa, Eine neue Form der Umwandlung des Acetaldehyd durch gärende Hefe. VI. Mitteilung. Über Carboligase. Biochem. Z. 143: 553-65 (I923).

Neuberg, C., and Ringer, M., Über das Wesen der natürlichen Bernsteinsäurebildung. I. Mitteilung. Die Bernsteinsäuregärung $\alpha$-Ketoglutarsäure. Biochem. Z. 71: 226-36 (I9I4).

Neuberg, C., and Ringer, M., Über das Wesen der Näturlichen Bernsteinsäurebildung. Mitteilung. III. Die Überführung von Aldehydropropionsäure in Bernsteinsäure mittels Hefe. Biochem. Z. $91:$ I3 I-6 (19I8).

Neuberg, C., and Rosenthal, P., Über Zuckerfreie Hefegärung. XI. Weiteres zur Kenntnis der Carboxylase. Biochem.Z.51: I 28-42 (1913).

Neuberg, C., and Rosenthal, P., Fortgesetzte Untersuchungen über die Carboxylase. Biochem. Z. 61: I7 I-83 (I9I4).

Neuberg, C., and Schwenk, Erwin, Die Gärung der Dioxymaleinsäure. Biochem. Z. 71: $10_{4}^{-13}$ (1915). 
Neuberg, C., and Schwenk, Erwin, Phytochemische Reduktionen. X. Reduktion von Glykolaldehyd zu Äthylenglykol. Biochem. Z. 71: I I4-7 (I9I5).

Neuberg, C., and Schwenk, Erwin, Phytochemische Reduktionen. XI. Die Umwandlung von Äthyldisulfid in Äthylmercaptan Biochem. Z. 71: I I 8-2I (I9I5).

Neuberg, C., and Simon, E., Zur Kenntnis der biochemischen Acytoinsynthese. VIII. Mitteilung über Carboligase. Biochem. Z. 156: $374^{-8}$ (1925).

Neuberg, C., and Simon, E., Über die Dismutation des Methyläthylacetaldehyds. Biochem. Z. 174: 452-6 (1926).

Neuberg, C., and Simon, E., Beiträge zur Biochemie des Asymmetrieproblems. (Asymmetrische Dismutation). Biochem. Z 179: 443-50 (I926).

Neuberg, C., and Simon, E., Die Verwirklichung der Phytochemische Reduktionen durch Bakterien. Biochem. Z. 190: 226-3I (I927).

Neuberg, C., and Simon, E., Über die Quantitative Dismutation des Methylglyoxals zu Milchsäure durch den Bacillus Delbriicki sowie durch das Bacterium lactis aerogenes nebst Versuchungen über das Abweichende stereochemische Verhalten des Phenylglyoxals bei dieser Reaktion. Biochem.Z. 186: 33I-6 (1927).

Neuberg, C., and Simon E., Alkoholische Zuckerspaltung durch Essigbakterien. Biochem. Z. 197: 259-60 (1928).

Neuberg, C., and Simon, E., Über Verschiedenheit der Vorgänge bei der alkoholischen Zuckerspaltung. Biochem. Z. 199: 23247 (1928).

Neuberg, C., and Steen bock, H., Über die Bildung höherer Alkohole aus Aldehyden durch Hefe. I. Übergang von Valeraldehyde in Amylalkohol. Biochem. Z. 52: 494-503 (1913).

Neuberg, C., and Steenbock, H., Über die Bildung höherer Alkohole aus Aldehyden durch Hefe. II. Weiteres über die Entstehung von Amylalkohol aus Valeraldehyd, insbesondere über die enzymatische Natur dieser Reaktion. Biochem. Z. 59: I89-92 (I9I4). Neuberg, C., and Welde, E., Phytochemische Reduktionen. VIII. Die Überführung des Formaldehyds in Methyl Alkohol. Biochem. Z. 67 : $104-10$ (I9I4).

Neuberg, C., and Welde, E., Phytochemische Reduktionen. II. Umwandlung aliphatischer Nitrokörper in Aminoverbindungen. Biochem.Z.62: 470-6 (1914). 
Neuberg, C., and Welde, E., Phytochemische Reduktionen. III. Umwandlung aromatischer und fettaromatischer Aldehyds in Alkohole. Biochem. Z. 62: 477-8I (I9I4).

Nicolai, W., and Kageura, N., Über den Fermentstoffwechsel der Bakterien. V. Untersuchungen am Staphylokokkus. Biochem. Z. 196: $2{ }_{4} 6-56$ (I928).

Nill, Walter, Neue einheimische Rhizopusartens als Stärkeverzuckerer. Centr. Bakt. Parasitenk. II. Abt. 72: 2I-38 (I927).

Nilsson, Ragnar, and SAndberg, Erik, Zur kenntniss der Garüngsspaltengen in Milchsäurebacterien und in Hefe. Biochem. Z. 174: 106-16 (1926).

Northrop, John H., Ashe, Lauren H., and Morgan, R. R., A fermentation process for the production of acetone and ethyl alcohol. J. Ind. Eng. Chem. 11: 723-7 (1919).

Northrop, John H., Ashe, Lauren H., and Senior, James K., Biochemistry of Bacillus acetoethylicum with reference to the formation of acetone. J. Biol. Chem. 39: I-2 I (I9I9).

Онта, Коньні, Zur Kenntnis der biochemischen Reduktionsvorgänge in Hefezellen. Die Umwandlung von Isobutylaldehyd und Isobutylalkohol und von Enanthol in n-Heptylalkohol. Biochem. Z. 59: $183-7$ (1914).

Omelianski, V., Sur la fermentation de la cellulose. Compt. rend. 121: 653-5 (I895).

Oppenheimer, Max, Über die Bildung von Milchsäure bei der Alkoholischen Gärung. Z. physiol. Chem. 89: 45-62 (I9I3).

Oppenheimer, Max, Über die Bildung von Glycerin bei der alkoholischen Gärung. Z. physiol. Chem. 89: 63-77 (1913).

Orla-Jensen, Biologische Studien über den käsereifungsprozess unter spezieller Berücksichtigung der flüchtigen Fettsäuren. Landw. Jahrbuch, Schweiz, 18: $3^{\mathrm{I}} 4^{-}+00$ (1904).

Ostermalder, A., Production of lactic acid by acetic acid bacteria. Z. ges. Brau., 37: 35 (I9I4). After Chem. Absts. 9, I 493 (I9I5). Owen, WM. L., A study of the formati. $n$ of gum levan from sucrose. Louisiana Planter, 71: $33^{1-3}, 353^{-4}, 373^{-4}$ (1923).

PARIS, G., La fase aldeidica nella fermentazione alcolica. Staz. sper. agrar. Italiane 55: 389-406 (I 922).

Patrick, R., Werkman, C. H., and Hixon, R. M., Evidence of xylanase production by an actinomyces. J. Bact. 19: 16 (I930).

Pederson, Carl S., and Breed, Robert S., The fermentation of glucose by organisms of the genus Serratia. J. Bact. 16: 16385 (1928). 
Perold, A. J., Untersuchungen über Weinessigbakterien. Centr. Bakt. Parasitenk. II. Abt. 24: 13-54 (I909).

Peters, W. L., Die Organismen des Sauerteigs und ihre Bedeutung für die Brotgährung. Bot. Zeit. 47 : 420-3 I (I889).

PÉré, M. A., Fermentation lactique des corps sucrés par le coli-bacille du nourrisson. Ann. Inst. Pasteur 12: 63-72 (1898).

Peterson, W. H., and Fred, E. B., The fermentation of glucose, galactose, and mannose by Lactobacillus pentoaceticus, n. sp. J. Biol. Chem. 42: 273-87 (I920).

Peterson, W. H., and Fred, E. B., Fermentation of fructose by Lactobacillus pentoaceticus, n. sp. J. Biol. Chem. 41: 43 I-50 (I920).

Peterson, W. H., and Fred, E. B., The production of acetaldehyde by certain pentose-fermenting bacteria. J. Biol. Chem. 44: 2945 (1920).

Peterson, W. H., Fred, E. B., and Marten, E. A., The effect of molecular complexity on the end-products formed by Clostridium thermocellum. J. Biol. Chem. 70: 309-17 (1926).

Peterson, W. H., Fred, E. B., and Schmidt, E. G., The fermentation of pentoses by Bacillus granulobacter pectinovorum. J. Biol. Chem. 60: 627-3I (1924).

Peterson, W. H., Pruess, L. M., and Fred, E. B., The proteolytic action of certain lactic acid bacteria. J. Bact. 15: $165-78$ (1928).

Petersson, G. Troili, Einzellkultur von langsam wachsenden Bakterienarten, speziell der Propionsäurebakterien. Centr. Bakt. Parasitenk. II Abt. 42: 526 (I9I5).

Pigulevskit, G., and Charik, N., Zersetzung des Olivenöls unter den Einfluss der vitalen Tätegkeit einiger Mikroorganismen; Umwandlung von Oleinsäure in Ketostearinsäure. Biochem. Z. 200: 20I-IO (I928).

Puriewitsch, K., Über die Spaltung der Glycide durch die Schimmelpilze. Ber. deut. botan. Ges., 16: 368-77 (1898).

Quastel, J. H., On the fermentation of the unsaturated dicarboxylic acids. Part I, Fumaric acid. Biochem. J. 18: 365-80 (I924).

Quastel, J. H., XXIV. Dehydrogenations produced by resting bacteria. IV. A theory of the mechanism of oxidations and reductions in vivo. Biochem. J. 20: $166-94$ (1926)

Quastel, J. H., The study of "resting" or non-proliferating bacteria. J. Hyg. 28: $139-46$ (1928).

Quastel, J. H., Stephenson, Marjory, and Vhetham, Margaret D., Some reactions of resting bacteria in relation to anaerobic growth. Biochem. J. 19: 304-I7 (I925). 
Quastel, J. H., and IVhetham, Margaret, D., The equilibria existing between succinic, furmaric, and malic acids in the presence of resting bacteria. Biochem. J. 18: 519-34 (1924).

Quick, Armand J., and Kahn, Morton C., The fermentation of glycuronic acid by certain bacteria. J. Bact. 18: I33-7 (1929). Raistrick, Harold, and Clark, Anne B., On the mechanism of oxalic acid formation by Aspergillus niger. Biochem. J. 13: 32944 (I9I9).

Rimini, G., Pyruvic acid as an intermediate product of alcoholic fermentation. Ann. Chim. Applicata 16: 488-90 (1926).

Robins, Wm. J., and Lathrop, E. C., The oxidation of vanillin to vanillic acid by certain soil bacteria. Soil Science 7: $475^{-85}$ (1919!.

Robison, R., and Morgan, W. T. J., Trehalosemonophosphoric ester isolated from the products of fermentation of sugars with dried yeast. Biochem. J. 22: 1277-88 (I928).

Rona, Eliza beth, I. Über die Reduktion des Zimtaldehyds durch. Hefe. II. Vergärung von Benzylbrenztraubensäure. Biochem.

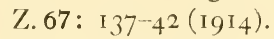

Rosenblatt, M., and Mordkowitsch, M., Ueber den Einfluss einiger Metalle auf die Essiggärung. Deut. Essigind. 33: 28 I-2 (1929).

Rosenfeld, L., Über die Phytochemische Reduktion des $\alpha, \alpha, \beta$ Trichlorbutylaldehyds zum 2, 2, 3-Trichlorbutanol Biochem. Z. 156: 54-7 (1925).

Rothenbach, F., Die Schnellessig bakterien. Wochensch. Brau. 16: $4^{\mathrm{I}-4}, 5^{8-9}, 70-2$, 100-2 (1899).

Rothenbach, F., Zur Systematik der Essigbakterien. Deutsche Essigind. 10: 193-4 (1906).

Rothenвach, F., Fortzüchtung von Reinzucht-Essigbakterien und ihre Uebertragung in den Betrieb. Deutsche Essigind. 10: 162$3,169-70$ (1906).

Rothenbach, F., Untersuchungen über die im Gärungsessig enthaltenen Enzyme und über die in der Fssigessenz enthaltenen Beimischungen. Die Deutsche Essigind. 13: 29.3 (1909).

Rothenbach, F., and Eberlein, L., Zu der Enzymgärung der Essigpilze. Die deutsche Essigind. 9: 23.34 (1905).

Ruhland, W., Über Arabinbildung durch Bakterien und deren Beziehung zum Gummi der Amygdaleen. Ber. deut. Bot. Ges. 24: $393^{-4}$ ㅇ ( ( 906$)$. 
SAITo, K., Ein Beispiel von Milchsäurebildung durch Schimmelpilze Centr. Bakt. Parasitenk. II Abt., 29: 289-90 (I9I I).

Santomauro, Pietro, Über die phytochemische Reduktion des Methyl- $\alpha$-chlorä thyl-ketons. Biochem. Z. 151: 48-50 (I924). Schardinger, Franz, Über eine neue optisch active Modification der Milchsäure durch bacterielle Spaltung des Rohrzuckers erhalten. Monatsh. 11: 545-59 (I890).

Scheffer, M. A., De suikervergisting door Bacteriën der Coli group. Thesis, Delft (1928).

Schmidt, Dorothea, Über die Pilzstärke (Amylose) bei Aspergillus niger $\mathrm{v}$. Tgh. und einige Bemerkungen über ihren diastatischen Abbau. Biochem. Z. 158: 223-52 (I925).

Schreyer, R., Über die Citronensäure-Bildung aus Glykonsäure durch Aspergillus. Ber. 58B : 2647 (1925).

Schreyer, R., Säuerungsversuche mit den Pilz Aspergillus fumaricus. Biochem. Z. 202: I 3 I-56 (I928).

Sclavo, A., and Gosio, B., Sur une nouvelle fermentation de l'amidon. Arch. ital. biol. 14: I 54-6 (1891).

Seifert, W., Beiträge zur Physiologie und Morphologie der Essigsäurebakterien. Cent. Bakt. Parasitenk. II Abt. 3: 337-49 (1897).

Seifert, W., Beiträge zur Physiologie und Morphologie der Essigsäurebakterien. Cent. Bakt. Parasitenk. II Abt. 3: 385-99 (I 897).

Sen, H. K., Über die carboxylatische Spaltung der Di-methylbrenztraubensäure und die Herstellung der $\alpha$-Keto-iso-valeriansäure. Biochem.Z. 143: 195-200 (1923).

SEn, H. K., Über die Gärung der $\alpha$-Keto-n-capronsäure. Biochem. Z. 140: 447-52 (1923).

SEN, H. K., Die biochemische Unwandlung von unsymmetrischem Di-chlor-acetone in optisch-activen $\alpha, \alpha$-di-chlor-iso-propylalkohol. Biochem. Z. 151: 5I-3 (I924).

Sherman, J. M., The cause of eyes and characteristic flavor in Emmental or Swiss cheese. J. Bact. 6: 379-9I (I92 I).

Sherman, J. M., and Shaw, R. H., The propionic acid fermentation of lactose. J. Biol. Chem. 56: 695-700 (1923).

Simonart, P., Lactic Fermentation. Bull. trimestr. assoc. élèves école sup. brasserie univ. Louvain 29: 75-8 I (1929). After Chem. Absts. 23: 4766 (I929).

Sjöвerg, KRUit, Spaltung der Stärke mit Saccharomyces Sake, Z. physiol. Chem. 162: 223-37 (1927). 
Slack, H. J., Notes on the Vinegar Plant. Quarterly Journal of Microscopical Science-New Series, 5: I1-5 (1865).

Smit, JAn, Von dem Stoffwechsel und der Verbreitung der Gärungssarcinen (Sarc. ventriculi, Goodsir und Sarc. maxima Lindner). Proc. Acad. Sci. Amsterdam. 31: 42I-32 (I 928).

Söhngen, N. L., Einflusz von Kolloiden auf Mikrobiologische Prozesse. Cent. Bakt. Parasitenk. II Abt. 38: 62 I-47 (1913).

Speakman, Horace B., Molecular configuration in the sugars and acid production by Bacillus granulobacter pectinovorum. J. Biol. Chem. 58: 395-413 (1923).

Speakman, Horace B., The biochemistry of acetone formation from sugars by Bacillus acetoethylicum. J. Biol. Chem. 64: $4^{\mathrm{I}-52}$ (1925).

Struyk, A. P., Onderzoekingen over de alcoholische gisting. 'Thesis, Delft ( 1928$)$.

Sumiki, Y., The products of fermentation of glucose by Oidium lupuli. Bull. Agr. Chem. Soc., Japan 2: $153-5$ (1926).

Supniewski, J., Recherches sur la transformation des combinaisons carbonies par le Bacille pyocyanique. Compt. rend. soc. biol. 89: $1377-9$ (1923).

Supniewski, J., Der Stoffwechsel der zyklischen verbindungen bei Bacillus pyocaneus. Biochem. Z. 146: 522 (1924).

Takahashi, T., Studies on the microorganisms of "Tanezu" (Japanese vinegar ferment). Journ. of the College of Agric. Imp. Univ. of 'Tokyo. 1: 103 (1909).

Takahashi, T., and Sakaguchi, K., The physiology of Rhizopus Species. Bull. Agr. Chem. Soc. Japan., 3: 35-45 (1927).

Tамiya, I. H., Metabolism of Aspergillus oryzae. Acta Phytochim. 3: 51-173 (1927). After Chem. Absts.

Tauson, V. O., Bacterial oxidation of crude oils. Neftyanoe Khozyaistvo 14: 220-30 (1928). After Chem. Absts. 23: I 43 I (I929).

Thompson, James, The chemical action of Bacillus cloacae (Jordon) on glucose and mannitol. Proc. Roy. Soc. London (B) 84: 500-4 (I9I2).

Томіта, M., Über die Umsetzung der d-Galaktose nach der Zweiten Vergärungsform. Biochem. Z. 121: 164-6 (1921).

Tomoda, Y., Production of glycerol by fermentation. IV. Dissociation of acetaldehyde-sodium bisulfite complex in alkaline solution. J. Soc. Chem. Ind. (Japan) 30: 747-59 (1927). 
Tomoda, Y., Production of glycerol by fermentation. VI. Influence of sugar concentration upon the yield of glycerol. Chem. News. 137: 265-6 (1928).

Traetta-Mosca, F., and Preti, M., Azione dell' Aspergillus glaucus sulla glicerina. Gazz. chimica italiana 51: II, 269-77 (I92I).

Traetta-Mosca, F., L'acido piruvico nella fermentazione alcoolica. Ann. chim. applicata, 16: I67-9 (1926).

Traetta-Mosca, F., Pyruvic acid as intermediate product of alcoholic fermentation. Ann. chim. applicata 17: 59-60 (1927). After Chem. Absts. 20: 3059 (1927).

Trillat, A., Sur la formation de l'aldehyde acetique dans les fermentations alcooliques. Compt. rend. 146: $654^{-7}$ (1908).

Trillat and Sauton, Étude sur le rôle des levures dans l'aldehydification de l'alcool. Compt. rend. 147: 77-80 (1908).

U. S. Patent i,625,732. Butyric acid. April i9, I929.

U. S. Patent, i,668,8 I 4. "Butyl-acetonic" fermentation. May 8, I929.

U. S. Patent I,696,022. Butyl alcohol and acetone by fermentation. Dec. I $8,1929$.

U. S. Patent i,698,800. Glycerol by fermentation. Jan. i 5. See Fr. Patent 636, I 2 I.

U. S. Patent I,703,272. Yeast cultivation in treated waste sulfite liquor. Feb. 26, 1929.

U. S. Patent 1,725,083. Butyl and isopropyl alcohols. Aug. 20, I 929.

U. S. Patent 1,725,084. Fusel Oil by Fermentation. Aug. 20, I 929.

U. S. Patent 1,725,363. Glycerol by fermentation. Aug. 20, i 929.

U.S. Patent i,726,768. Lactates. Sept. 3, I929.

U. S. Patent I,727,223. Use of peroxidized compounds to stimulate fermentations. Sept. 3, I 929.

VAn Der Lek, J. B., Vibrio agarliquefaciens Gray. Nederland Tijdschr. Microbiol, Serol. 3: 276-80 (1 929).

Vandevelde, G., Studien zur chemie des Bacillus subtilis. Z. physiol. Chem., 8: 367-90 (i $88_{4}$ ).

VAN Niel, C. B., The propionic acid bacteria. Thesis, Haarlem (1928).

Viljoen, J. A., Fred, E. B., and Peterson, W. H., The fermentation of cellulose by thermophilic bacteria. J. Agr. Sci. 16: I-I7 (I926).

Villiers, A., Sur la transformation de la fécule en dextrine par le ferment butyrique. Compt. rend. 112: $435-7$ (I89I). 
Virtanen, Artturi I., Über die Propionsäuregärung. Soc. Sci. Fennica, Commentationes Physico-Math. I, No. 36, I-23 (1923). Virtanen, Artturi I., and Barlund, Brita, Die Oxydation des Glycerins zu Dioxyaceton durch Bakterein. Biochem. Z. 169: $169-77$ (1926).

Virtanen, A. I., and Karström, H., Quantitative Enzymbestimungen an Mikroorganisms. I. Der Katalasegehalt der Bakterien. Biochem. Z. 161: 9-46 (1925).

Virtanen, A. I., and Karström, H., Über die Milchsäuregärung. Z. physiol. Chem. 174: I-27 (I928).

Virtanen, A. I., and Simola, P. E., Über die Gärung des Zuckers durch Coli-Aerogenes Bakterien. I. Z. physiol. Chem. 163: 284-93 (1927).

Virtanen, A. I., Wichmann, E., and Lindström, B., Über die Milchsäuregärung. IV. J. physiol. Chem. 166: 2I-38 (I927).

Visser't Hooft, F., Biochemische onderzoekingen over het geslacht Acetobacter. Thesis, Delft (I925).

Voisenet, E., Sur une Bactèrie de l'eau végétant dans les rins Amers capable de deshydrater la glycerine glycero-réaction. Ann. Inst. Pasteur, 32: 476 (1918).

Walker, Thomas Kennedy, Subramaniam, Vira, and Challenger, Frederick, The mechanism of the formation of citric and oxalic acids from sugars by Aspergillus niger. Part II. J. Chem. Soc. 3044-54 (1927).

WALPOLE, G. S., The action of Bacillus lactis aerogenes on glucose and mannitol. Part II. The investigation of the $2: 3$ butanediol and the acetylmethylcarbinol formed; the effect of free oxygen on their production; the action of $B$. lactis aerogenes on fructose. Proc. Roy. Soc. (London) 83 B : 272-86 (igII).

Wenmer, C., Entstehung und physiologische Bedeutung der Oxalsäure im Stoffwechsel einiger Pilze. Bot. Zeit. 49: $233^{-46,249^{-}}$ $5^{8}, 271-80,289^{-9} 9^{8}, 305^{-1} 3,321-32,33^{6}-46,353^{-6} 3,369^{-82}$, $3^{8} 5^{-9} 9^{6}, 40 \mathrm{I}-7,4^{1} 7^{-28}, 433^{-9}, 449^{-55}, 4^{6} 5^{-7} 8$ (I 89 I).

Wenmer, C., Über zwei weitere freie Citronensäurebildende Pilze. Chem. Zeit, 21: 1022-3 (1897)

Wenmer, C., Kleine mykologische Mitteilungen. I. Zur Oxalsäuregärung durch Aspergillus niger. Centr. Bakt. Parasitenk. II Abt. 3: $102-4$ (1897).

Wenmer, C., Über Citronensäurebildung aus Glycerin durch Pilze. Chem. Zeit. 37: 37-9 (I9I3). 
Wenmer, C., Über Fumarsäure-Gärung des Zuckers. Ber. 51: II, I $663-8$ (I9I8).

Wenmer, C., Oxalsäure und Citronensäure-Entstehung in ihrer gegenseitingen Beziehung bei verschiedenen Rassen des Pilzes Aspergillus niger. Ber. 57: II, I659-65 (1924).

Wehmer, C., Bildung von Citronensäure aus Glykonsäure durch Pilze. Ber. 58: II, 26I6-I9 (I925).

Wehmer, C., Die Gattung "Citromyces" und die Zitronensäurebildung. Centr. Bakt. Parasitenk. II Abt. 73: I6I-2 (I928). Wenmer, C., Abnahmedes Säuerungsvermögens und Änderung der Säure bei einem Pilz. Gluconsäure statt Fumarsäure- Gärung.

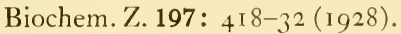

Weiss, S., and Pogany, J., Untersuchungen über den abbau von Acetessigsäure durch Colibacillen. Z. ges. exptg. Med. 51: 26I6 (1926).

Werkman, C. H., Hixon, R. M., Fulmer, E. I., and Rayburn, C. H., Production of propionic acid from pentoses. Proc. Iowa Acad. Sci. 36: (1929).

Werkman, C. H., and Kendall, Sara, Physiology and Classification of the Propionic acid bacteria. J. Bact. 19: i 8 (I930).

IVertheimer, Ernst, Zuckerbindung und anschliessende synthetische Vorgänge durch Hefezellen. Fermentforschung, 11: 22-36 (1929).

Weyer, E. R., and Rettger, L. F., A comparative study of six different strains of the organism commonly concerned in large-scale production of butyl alcohol and acetone by the biological process.

J. Bact. 14: 399-424 (1927).

Wheтнам, M. D., Origin of acetaldehyde in bacterial and animal metabolism. Australian J. Exptl. Biol. Med. Sci. 4: 35-56 (I927).

Windisch, Fritz, Beziehungen zwischen oxybiontischem und anoxybiotischem Stoffwechsel der Kulturhefen. Z. physiol. Chem. 179: 88-98 (1928).

Winogradsky, S., Clostridium Pastorianum, seine Morphologie und seine Eigenschaften als Buttersäureferment. Centr. Bakt. Parasitenk. II Abt. 9: $+3-5+$ (I902).

Wojtriewicz, A., Propionsäuregärung im Emmental Käse russischer Fabrikation. Centr. Bakt. Parasitenk. II Abt., 59: 333 (1923). Woolf, Barnet, Some enzymes in $B$. coli communis which act on fumaric acid. Biochem. J. 23: $47^{2-82}$ (I 929 ).

YAMADA, MASAKaZU, The mechanism of the fruit-ester formation by Willia anomala. Bull. Agr. Chem. Soc. Japan 3: 73-6 (1927). 
Young, E. Gordon, The decomposition of glucose by bacteria. J. Biol. Chem. 43: 59 (I924).

Young, WM. J., The hexosephosphate formed by yeast-juice from hexose and phosphate. Proc. Royal Soc. 81: B., 528-45 (I909). Yuill, Јонn L., Alcoholic fermentation by Aspergillus flavus, Brefeld. Biochem. J. 22: I 504-7 (I 928 ).

Zeissler, J., and Rassfeld, L., Die Kohlenhydrat spaltung durch die anaëroben Bazillen. Centr. Bakt. Parasitenk., I. Abt., 110: 24-44 (1929).

Zerner, Ernst, Über den Chemismus der alkoholischen Gärung. Ber. 53: I, 325-34 (1920). 




\section{AN INDEX TO THE CHEMICAL ACTION OF MICROORGANISMS ON THE NON-NITROGENOUS ORGANIC COMPOUNDS}

was set, printed and bound by The Collegiate Press of Menasha, Wisconsin. The cover design is by The Decorative Designers of Chatham, New Fersey. The type face is Monotype Caslon Old Style. The type is set ten on twelve point, $337 E$. The type page is $25 \times 43$ picas. The end sheets are 80 pound $W$ hite Antique Tujtex. The jacket is $27 \times 36-80$ Gray Antique Construction. The text paper is $38 \times 50,140$ (7o lb.) Winnebago Text. The binding is Holliston's Waverly 283.

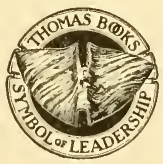

With THOMAS BOOKS careful attention is given to all details of manufacturing and design. It is the publisher's desire to present books that are satisfactory as to their physical qualities and artistic possibilities and appropriate for their particular use. THOMAS BOOKS will be true to those laws of quality that assure a good name and good will. 




